\title{
Construction of Thienothiophene and Thienofuran Ring Systems via Ring Expansion of Difluorothiiranes Generated from Dithioesters
}

\author{
Kohei Fuchibe, ${ }^{1}$ Ibuki Mukohara, ${ }^{1}$ Atsushi Yamada, ${ }^{1}$ Daisuke Miyazaki, ${ }^{1}$ \\ Ryo Takayama, ${ }^{1}$ and Junji Ichikawa ${ }^{1}$. \\ kfuchibe@chem.tsukuba.ac.jp,junji@chem.tsukuba.ac.jp \\ ${ }^{1}$ Division of Chemistry, Faculty of Pure and Applied Sciences \\ University of Tsukuba, Tsukuba, Ibaraki 305-8571, Japan
}

\section{— Supporting Information -}

\section{Table of Contents}

1. General Statements $\quad \ldots \ldots \ldots . .6$ S2

2. Structures of Difluorothiirane and Its Li Complex $\quad$......... S4

3. Preparation of Thiophene- and Furancarbodithioates $\quad$ …...... S6

4. Optimization of Reaction Conditions $\quad \cdots \cdots . . .6$ S9

5. Measurement of Half-Life Times of Carbene Sources $\quad$.......... S11

6. Study on the Effect of Li Lewis Acids $\quad$ …..... $\quad$ S13

7. Typical Procedures $\quad \cdots \cdots \cdots . . .6$ S15

8. Spectral Data of Thiophene- and Furancarbodithioates $\quad \cdots \cdots \cdots . . . \quad$ S16

9. Spectral Data of Thienothiophenes, Difluoroalkenes, Thienofurans, and Thienothienofurans $\quad$.......... $\quad$ S21

10. ORTEP Diagrams of Products $\quad \cdots \cdots \cdots . . .627$

11. NMR Spectra of Substrates and Products $\quad$ …...... S30 


\section{General Statements}

\section{1-1. Solvents and Reagents}

Nitromethane was distilled from $\mathrm{P}_{2} \mathrm{O}_{5}$ and then stored over MS 4A. Lithium, sodium, potassium, and cesium bromodifluoroacetates were prepared from commercially available ethyl bromodifluoroacetate by the method described in our previous paper. ${ }^{[1]}$ These carbene sources were hygroscopic and thus stored in a glovebox. Molecular sieves 3A, 4A, 5A, and 13X (powder) were purchased from Merck KGaA, dried under microwave irradiation ( $3 \mathrm{~min}$ ), and further flame dried in a reaction vessel just before use. Lithium hexafluorophosphate was purchased from FUJIFILM Wako Pure Chemical Corporation and used as recieved. Phenyl chlorodithioformate was purchased from Merck KGaA and used as received. 2,4-Bis(4methoxyphenyl)-1,3-dithia-2,4-diphosphetane 2,4-disulfide (Lawesson's reagent) was purchased from Chem Inpex International, Inc. and used as received. 1,1,1,3,3,3-Hexafluoro-2,2-di( $p$-tolyl) propane and $\alpha, \alpha, \alpha$-trifluorotoluene (internal standards for ${ }^{19} \mathrm{~F}$ NMR quantitative analysis) were purchased from Tokyo Chemical Industry Co., Ltd. and used as recieved.

\section{1-2. Purification}

Column chromatography and preparative thin layer chromatography (PTLC) were conducted on silica gel (Silica Gel 60 N, Kanto Chemical Co., Inc. for column chromatography and Wakogel B-5F, FUJIFILM Wako Pure Chemical Corporation for PTLC). Purification was also performed by preparative HPLC (GPC), using a JAI LC-908 instrument (Jaigel-2H, $\mathrm{CHCl}_{3}$ ).

Thiophene- and furancarbodithioates were recrystalized from hexane prior to measuring their melting points. Thienothiophenes, thienofurans, and thienothienofurans were recrystalized from hexane/dichloromethane (ca. 8:1) prior to measuring their melting points.

\section{1-3. Reaction Vessels and Microwave Apparatus}

All reactions were performed using standard Schlenk techniques. Micrawave irradiation was performed using a CEM Discover apparatus in a sealed vessel.

\section{1-4. Analyses}

IR spectra were recorded on a Horiba FT-300S spectrometer by the attenuated total reflectance (ATR method). NMR spectra were recorded on Bruker Avance 500 or Jeol JNM

${ }^{1}$ Fuchibe, K.; Aono, T.; Hu, J.; Ichikawa, J. Org. Lett. 2016, 18, 4502-4505. 
ECS-400 spectrometers in $\mathrm{CDCl}_{3}$ at 500 or $400 \mathrm{MHz}\left({ }^{1} \mathrm{H} \mathrm{NMR}\right)$, at 126 or $101 \mathrm{MHz}\left({ }^{13} \mathrm{C} \mathrm{NMR}\right)$, and at 470 or $376 \mathrm{MHz}\left({ }^{19} \mathrm{~F}\right.$ NMR). Chemical shifts were given in ppm relative to internal $\mathrm{Me}_{4} \mathrm{Si}$ (for ${ }^{1} \mathrm{H}$ NMR: $\delta=0.00$ ), $\mathrm{CDCl}_{3}$ (for ${ }^{13} \mathrm{C} \mathrm{NMR}: \delta=77.0$ ), and $\mathrm{C}_{6} \mathrm{~F}_{6}$ (for ${ }^{19} \mathrm{~F} \mathrm{NMR:} \delta=0.0$ ). Highresolution mass spectroscopy (HRMS) was conducted with a Jeol JMS-T100GCV spectrometer (EI, TOF). Elemental analyses (EA) were performed with a Yanako MT-3 CHN Corder apparatus. Single crystal X-ray structure analysis was performed on a Bruker APEXII ULTRA instrument equipped with a CCD diffractometer using Mo K $\alpha$ (graphite monochromated, 1 $=0.71073 \AA$ ) radiation. Melting points were measured with a Yanako Micro Melting Point Apparatus MP-J9, equipped with a heating stage. 


\section{Structures of Difluorothiirane and Its Li Complex}

\section{2-1. Structural Optimization}

Eight local minimums of difluorothiirane-lithium ion complex $\mathbf{2 a - L i - a - h}$ were obtained from thirteen initial structures ISa-m, using Gaussian16 program (Fig S1; DFT, B3LYP/6$\left.31 \mathrm{G}^{*}\right)$.

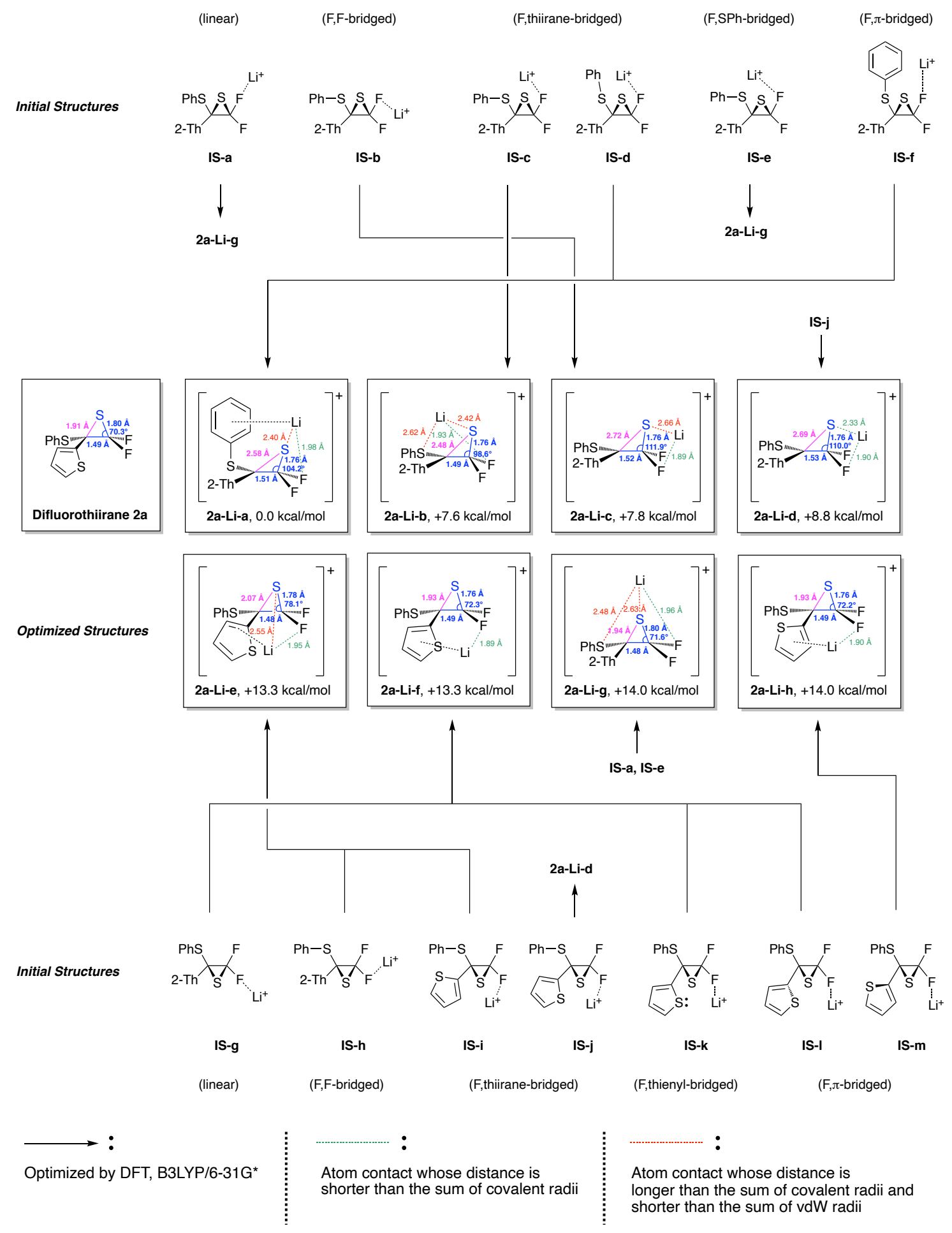

Fig S1. 
2-2. Optimized Structures of Difluorothiirane and Difluorothiirane- $\mathrm{Li}^{+}$Complex $\mathbf{2 a - L i - a}$

Structures of difluorothiirane (Fig S2) and difluorothiirane-Li complex 2a-Li-a (Fig S3) optimized by Gaussian16 program (DFT, B3LYP/6-31G*, Gaussian, Inc.) are shown. Structual parameters (bond lengths and angles) were calculated by Gaussian 16. Löwdin bond orders (LBOs) were calculated by Spartan 18 program (DFT, B3LYP/6-31G*, Wavefunction, Inc.).
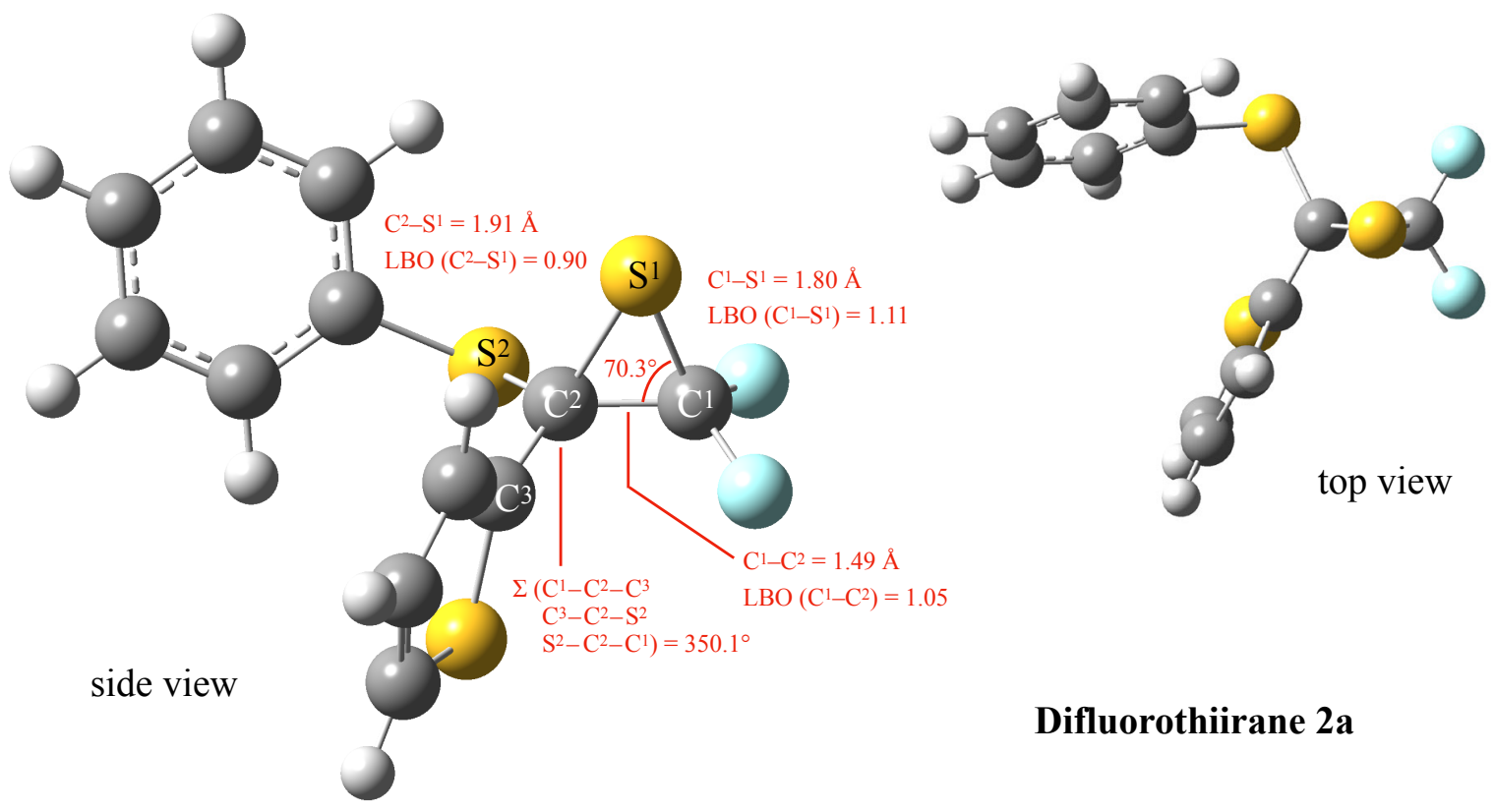

Fig S2.
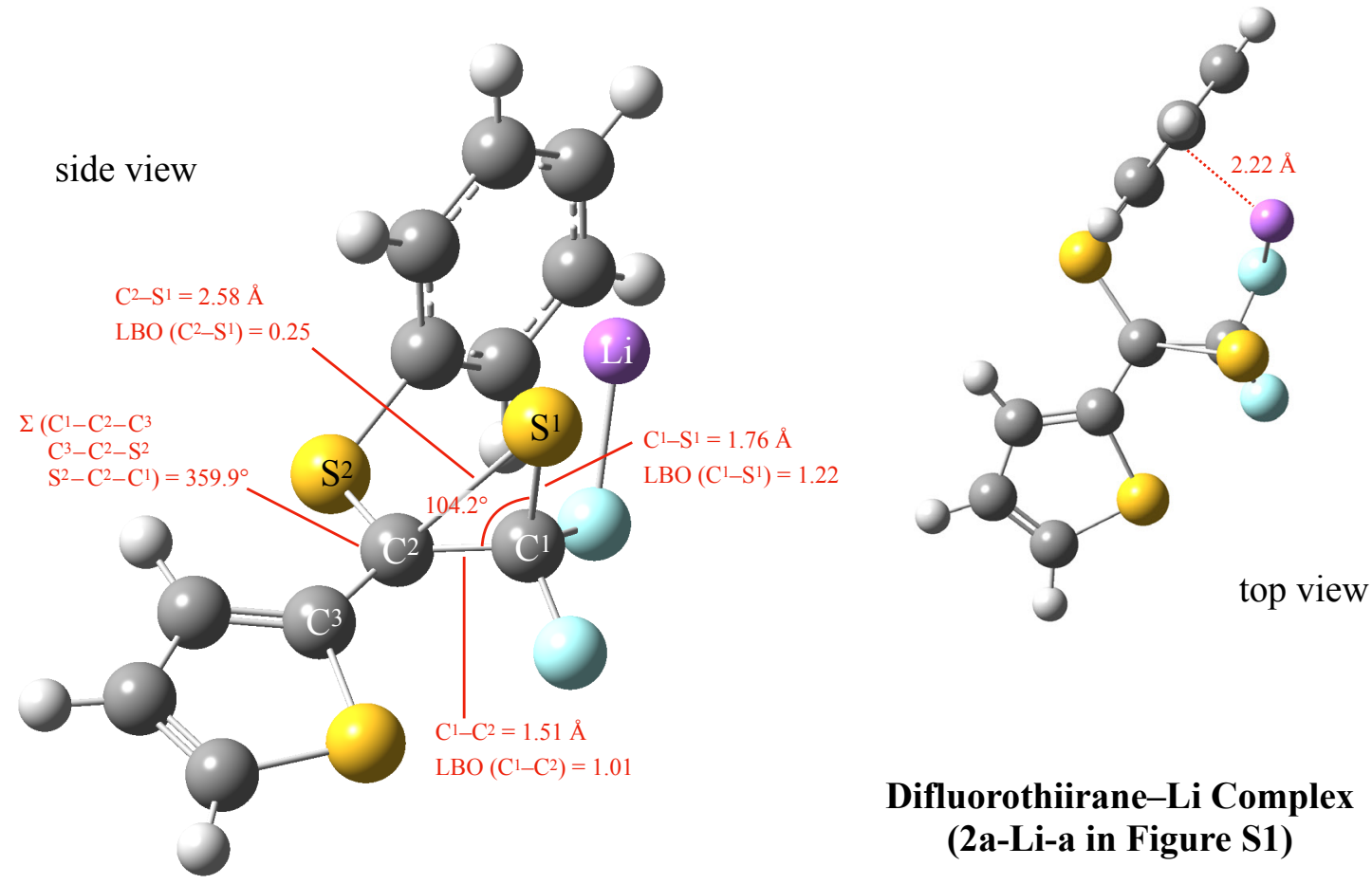

Fig S3.

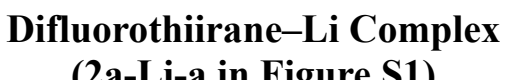

(2a-Li-a in Figure S1) 


\section{Preparation of Thiophene- and Furancarbodithioates}

Thiophene- and furancarbodithioates were prepared as reported (Method I) ${ }^{[2]}$ Dithioates were also prepared by our facile procedure (Method II).

3-1. Method I

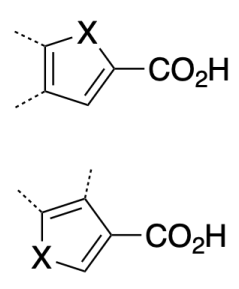

acids

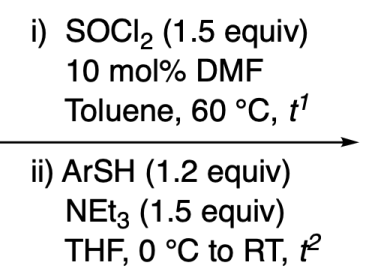

THF, $0^{\circ} \mathrm{C}$ to $\mathrm{RT}, t^{2}$<smiles></smiles><smiles>O=C(C1=CC=[Y]C1)c1c[Y]cc1</smiles>

thioates iii) Lawesson's reagent ( 0.75 equiv)

Toluene, reflux, $t^{3}$<smiles>Cc1cc(C(=S)[Se])cc(C)c1I</smiles><smiles></smiles>

dithioates 1

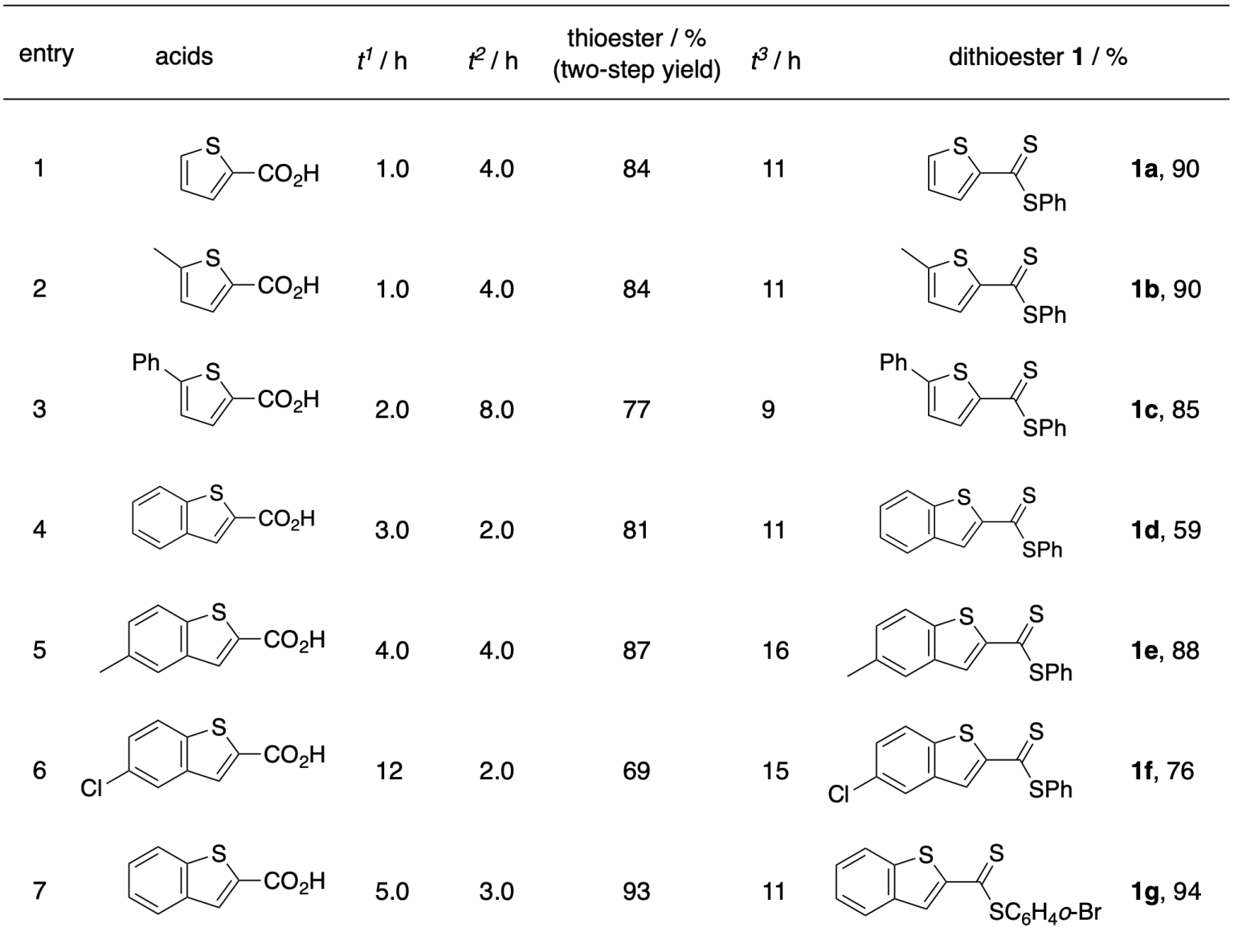

Table S1.

Takayama, R.; Yamada, A.; Fuchibe, K.; Ichikawa, J. Org. Lett. 2017, 19, 5050-5053. 


(1.0

\section{3-2. Method II}

Thiophenecarbodithiolates could be prepared more readily, using the corresponding Grignard reagents and commercially available phenyl chlorodithioformate. Preparation of carbodithioate 1a is described as a typical procedure.

To a THF suspension $(50 \mathrm{~mL})$ of magnesium $(1.34 \mathrm{~g}, 55.1 \mathrm{mmol})$ was added 2-bromothiophene $(4.8 \mathrm{~mL}, 50 \mathrm{mmol})$ at $0{ }^{\circ} \mathrm{C}$ over $10 \mathrm{~min}$. The suspension was stirred overnight and titration indicated that the concentration was $0.50 \mathrm{~mol} / \mathrm{L}$. To a THF solution (1 $\mathrm{mL})$ of phenyl chlorodithioformate $(43 \mu \mathrm{L}, 0.30 \mathrm{mmol})$ were successively added copper(I) 
iodide $(11 \mathrm{mg}, 0.060 \mathrm{mmol})$ and the THF solution of 2-thienylmagnesium bromide $(0.60 \mathrm{~mol} / \mathrm{L}$, $0.30 \mathrm{mmol})$ at $-78{ }^{\circ} \mathrm{C}$. The resulting solution was slowly warmed up to room temperature over $12 \mathrm{~h}$. Phosphate buffer $(\mathrm{pH}=7)$ was added to quench the reaction at room temperature. Organic materials were extracted with ethyl acetate three times. The combined extracts were washed with brine and dried over anhydrous $\mathrm{Na}_{2} \mathrm{SO}_{4}$. After removal of the solvent under reduced pressure, the residue was purified by column chromatography on silica gel (hexane) to give thiophenecarbodithioate $\mathbf{1 a}(60 \mathrm{mg}, 84 \%$ yield $)$ as red crystals.

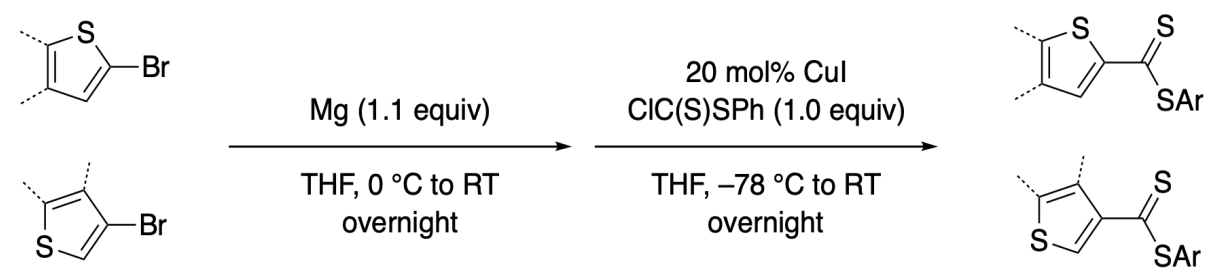

Scheme S1. 


\section{Optimization of Reaction Conditions}

4-1. Effect of Molecular Sieves and Water

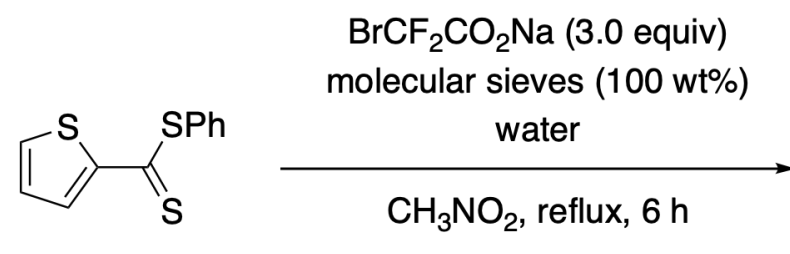

1a $(0.03 \mathrm{M})$

\begin{tabular}{ccccc} 
1a $(0.03 \mathrm{M})$ & \multicolumn{3}{c}{$\mathbf{3 a}$} & $\mathbf{4 a}$ \\
\hline entry & molecular sieves & water & $\mathbf{3 a}+\mathbf{4 a} / \%$, ratio $^{a}$ & $\mathbf{1 a}$ (recovery) $/ \%^{b}$ \\
\hline 1 & none & none & $53,98: 2$ & - \\
2 & MS 3A & none & $70,91: 9$ & - \\
3 & MS 4A & none & $77,87: 13$ & - \\
4 & MS 5A & none & $65,94: 6$ & - \\
5 & MS 13X & none & $73,97: 3$ & 31 \\
$6^{c}$ & none & 1.5 equiv & $46,>99:<1$ & 18 \\
$7^{c}$ & MS 4A ${ }^{d}$ & 1.5 equiv & $74,93: 7$ & - \\
\hline
\end{tabular}

${ }^{a}{ }^{19} \mathrm{~F}$ NMR yield based on $\mathrm{PhCF}_{3} \cdot{ }^{b}{ }^{1} \mathrm{H}$ NMR yield based on $\mathrm{CH}_{2} \mathrm{Br}_{2} \cdot{ }^{c} 20$ h. ${ }^{d} 200$ wt\%.

Table S2.

4-2. Effects of Counter Cations and Molecular Sieves Loadings

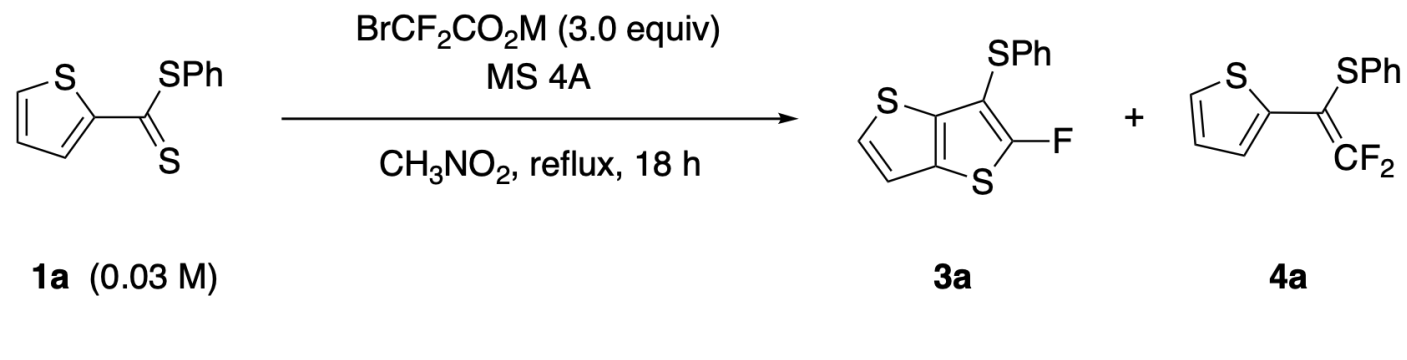

\begin{tabular}{cccccc}
\hline entry & $M$ & MS 4A/ equiv & $t / \mathbf{h}$ & 3a $+\mathbf{4 a} / \%$ ratio $^{a}$ & 1a (recovery) $/ \%^{b}$ \\
\hline 1 & $\mathrm{Li}$ & 100 & 7 & $73,97: 3$ & 10 \\
2 & $\mathrm{Li}$ & 200 & 18 & $78,3 \mathbf{a}$ only & - \\
3 & $\mathrm{Na}$ & 200 & 18 & $73,81: 19$ & - \\
4 & $\mathrm{~K}$ & 200 & 18 & $69,87: 13$ & - \\
5 & $\mathrm{Cs}$ & 200 & 18 & $41,88: 12$ & 49 \\
\hline
\end{tabular}

a ${ }^{19} \mathrm{~F}$ NMR yield based on $\mathrm{PhCF}_{3} .{ }^{b} \mathrm{H}$ NMR yield based on $\mathrm{CH}_{2} \mathrm{Br}_{2}$.

Table S3. 
4-3. Effects of Solvents and Concentrations

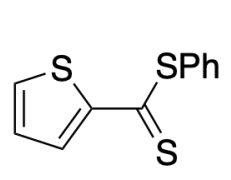

$1 \mathrm{a}$

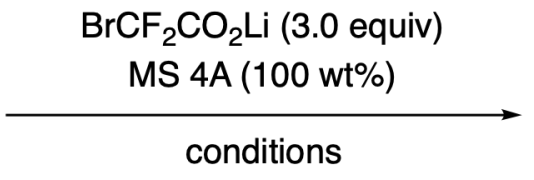

conditions

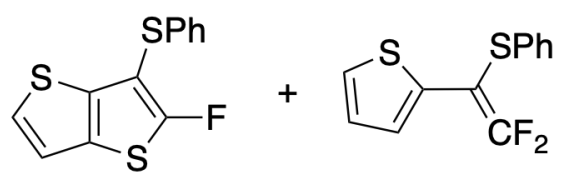

3a
$4 a$

\begin{tabular}{cllccccc}
\hline entry & \multicolumn{2}{c}{ solvent (DN) } & $\mathbf{1 a} / \mathrm{M}$ & temp & $t / \mathbf{h}$ & $\mathbf{3 a}+\mathbf{4 a} / \%$, ratio a & $\mathbf{1 a}$ (recovery) /\% $b$ \\
\hline 1 & $\mathrm{CH}_{3} \mathrm{NO}_{2}$ & $(2.7)$ & 0.10 & reflux & 10 & $65(94: 6)$ & 25 \\
2 & $\mathrm{CH}_{3} \mathrm{NO}_{2}$ & $(2.7)$ & 0.03 & reflux & 7 & $73(97: 3)$ & 10 \\
3 & $\mathrm{CH}_{3} \mathrm{NO}_{2}$ & $(2.7)$ & 0.01 & reflux & 6 & $72(3 \mathbf{a}$ only) & 17 \\
4 & $i-\mathrm{PrNO}_{2}$ & & 0.10 & 120 & 6 & $70(96: 4)$ & - \\
5 & $\mathrm{PhNO}_{2}$ & & 0.10 & 140 & 6 & $35(89: 11)$ & - \\
6 & $\mathrm{PhCN}^{(11.9)}$ & 0.10 & 120 & 6 & $64(38: 62)$ & 18 \\
7 & diglyme & $(19.5)$ & 0.10 & 120 & 6 & $55(45: 55)$ & - \\
8 & THF & $(20.0)$ & 0.10 & reflux & 6 & $57(33: 67)$ & - \\
9 & DMF & $(26.6)$ & 0.10 & 100 & 6 & - & 93 \\
10 & DMSO & $(29.8)$ & 0.10 & 120 & 6 & $4(3 a$ only) & 97 \\
\hline
\end{tabular}

${ }^{a}{ }^{19} \mathrm{~F}$ NMR yield based on $\mathrm{PhCF}_{3} .{ }^{b}{ }^{1} \mathrm{H}$ NMR yield based on $\mathrm{CH}_{2} \mathrm{Br}_{2}$. $\mathrm{DN}=$ donor number; diglyme $=\mathrm{CH}_{3}\left(\mathrm{OCH}_{2} \mathrm{CH}_{2}\right)_{2} \mathrm{OCH}_{3}$.

Table S4. 


\section{Measurement of Half-Life Times of Carbene Sources}

\section{5-1. Principle}

Half-life times of bromodifluoroacetates, $\mathrm{BrCF}_{2} \mathrm{CO}_{2} \mathrm{M}$, were estimated based on the assumption that the decomposition of these salts is expressed as follows:

$$
\mathrm{d}\left[\mathrm{BrCF}_{2} \mathrm{CO}_{2} \mathrm{M}\right] / \mathrm{d} t=-k\left[\mathrm{BrCF}_{2} \mathrm{CO}_{2} \mathrm{M}\right]
$$

, where $\left[\mathrm{BrCF}_{2} \mathrm{CO}_{2} \mathrm{M}\right]$ and $t$ are concentration of the salt determined and heating time, respectively, and $k$ is a constant. On the basis of this asumption, the concentration of the salt is expressed as follows:

$$
\left[\mathrm{BrCF}_{2} \mathrm{CO}_{2} \mathrm{M}\right]=\left[\mathrm{BrCF}_{2} \mathrm{CO}_{2} \mathrm{M}\right]_{0} \exp (-k t)
$$

, where $\left[\mathrm{BrCF}_{2} \mathrm{CO}_{2} \mathrm{M}\right]_{0}$ is the initial concentration. The recovery of the salt $Y$, which is experimentally obtained, can be expressed as follows:

$$
Y=\exp (-k t)
$$

, where $Y$ is defined as $Y=\left[\mathrm{BrCF}_{2} \mathrm{CO}_{2} \mathrm{M}\right] /\left[\mathrm{BrCF}_{2} \mathrm{CO}_{2} \mathrm{M}\right]_{0}$. The least square fitting of i) $\ln Y$ and ii) $t$ affords the constant $k$.

Using the above constant $k$, the half-life time of the salt $t_{1 / 2}(Y=1 / 2)$ can be calculated as follows:

$$
t_{1 / 2}=-(1 / k) \ln (1 / 2)
$$

\section{5-2. Procedure}

Measurement of the half-life time of lithium bromodifluoroacetate in the presence of MS $4 \mathrm{~A}$ is described as a typical procedure. To a mixture of lithium bromodfluoroacetate $(181 \mathrm{mg}, 1.00$ mmol), MS 4A (78 mg, powder), and 1,1,1,3,3,3-hexafluoro-2,2-di(p-tolyl)propane (an internal standard for ${ }^{19} \mathrm{~F}$ NMR quantitative analysis) was added acetonitrile $(10 \mathrm{~mL})$. After the resulting solution was refluxed for $5 \mathrm{~min}$ (an oil bath), an aliquot (ca. $0.8 \mathrm{~mL}$ ) of the reaction mixture was analyzed by ${ }^{19} \mathrm{~F}$ NMR, which revealed that $97 \%$ of the starting lithium bromodifluoroacetate remained. After each heating time $(15,30$, and $60 \mathrm{~min})$, an aliquot was analyzed in a similar manner. 
5-3. Results

$$
\underset{(0.10 \mathrm{M})}{\mathrm{BrCF}_{2} \mathrm{CO}_{2} \mathrm{M}} \quad \frac{(\mathrm{MS} 4 \mathrm{~A} 40 \mathrm{wt} \%)}{\mathrm{CH}_{3} \mathrm{NO}_{2} \text {, reflux or } 80^{\circ} \mathrm{C}} \text { recovery }(Y)
$$

\begin{tabular}{|c|c|c|c|c|c|c|}
\hline \multirow{2}{*}{$t / \min$} & \multicolumn{4}{|c|}{$Y / \%,{ }^{a}$ reflux } & \multicolumn{2}{|c|}{$Y / \%,{ }^{a} 80^{\circ} \mathrm{C}$} \\
\hline & $\mathrm{Li}$ & Li, MS 4A & $\mathrm{Na}$ & $\mathrm{K}$ & $\mathrm{K}$ & Cs \\
\hline 5 & quant & 97 & 74 & 6 & 32 & 20 \\
\hline 15 & ND & 82 & 50 & ND & 12 & ND \\
\hline 30 & quant & 70 & 34 & 1 & 6 & 3 \\
\hline 60 & 98 & 56 & 20 & ND & ND & ND \\
\hline
\end{tabular}

${ }^{a}{ }^{19} \mathrm{~F}$ NMR yield based on $p-\mathrm{Tol}_{2} \mathrm{C}\left(\mathrm{CF}_{3}\right)_{2}$. ND $=$ not determined.

Table S5.

\begin{tabular}{|c|c|c|c|c|}
\hline \multirow{2}{*}{$: \mathrm{CF}_{2}$ source } & \multicolumn{2}{|l|}{ reflux } & \multicolumn{2}{|c|}{$80^{\circ} \mathrm{C}$} \\
\hline & $k$ & $t_{1 / 2}$ & $k$ & $t_{1 / 2}$ \\
\hline $\mathrm{BrCF}_{2} \mathrm{CO}_{2} \mathrm{Li}$ & no decomposition & - & & \\
\hline $\mathrm{BrCF}_{2} \mathrm{CO}_{2} \mathrm{Li}+\mathrm{MS} 4 \mathrm{~A}(40 \mathrm{wt} \%)$ & $9.9 \times 10^{-3}$ & $70 \mathrm{~min}$ & & \\
\hline $\mathrm{BrCF}_{2} \mathrm{CO}_{2} \mathrm{Na}$ & $2.5 \times 10^{-2}$ & $28 \mathrm{~min}$ & & \\
\hline $\mathrm{BrCF}_{2} \mathrm{CO}_{2} \mathrm{~K}$ & $1.3 \times 10^{-1}$ & $5.5 \mathrm{~min}$ & $8.8 \times 10^{-2}$ & $7.9 \mathrm{~min}$ \\
\hline $\mathrm{BrCF}_{2} \mathrm{CO}_{2} \mathrm{Cs}$ & & & $1.0 \times 10^{-1}$ & $6.7 \mathrm{~min}$ \\
\hline
\end{tabular}

Table S6. 


\section{Study on the Effect of Li Lewis Acids}

6-1. Effect of Lithium Salts

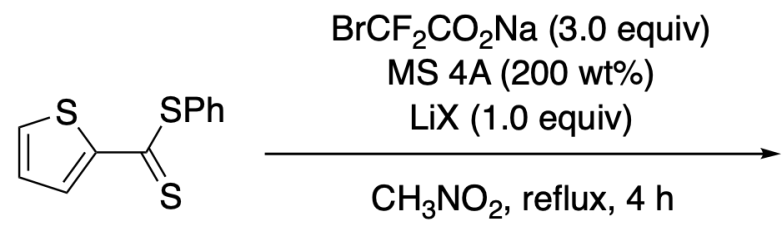

1a $(0.03 \mathrm{M})$

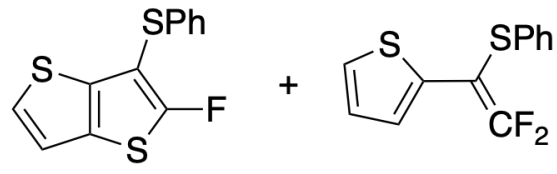

3a
$4 a$

\begin{tabular}{|c|c|c|c|c|}
\hline entry & LiX & $\begin{array}{l}\text { gas phase acidity } \\
\text { of } \mathrm{HX} / \mathrm{kcal} \mathrm{mol}^{-1} \text { a }\end{array}$ & $3 \mathbf{a}+4 \mathbf{a}(\text { ratio })^{b}$ & $1 \mathbf{a}$ (recovery) $^{c}$ \\
\hline 1 & none & - & $75(83: 17)$ & - \\
\hline 2 & $\mathrm{LiCl}$ & 324 & $82(83: 17)$ & - \\
\hline 3 & $\mathrm{LiBr}$ & 315 & $81(86: 14)$ & - \\
\hline 4 & LiOTf & 293 & $77(95: 5)$ & - \\
\hline 5 & $\mathrm{LiBF}_{4}$ & 288 & $87(94: 6)$ & - \\
\hline 6 & $\operatorname{LiNTf}_{2}$ & 287 & $78(99: 1)$ & - \\
\hline 7 & $\mathrm{LiPF}_{6}$ & 277 & $71(100: 0)$ & 11 \\
\hline
\end{tabular}

a Koppel, I. A.; Burk, P.; Koppel, I.; Leito, I.; Sonoda, T.; Mashima, M. J. Am. Chem. Soc. 2000, 122, 5114-5124; Leito, I.; Raamat, E.; Kütt, A.; Saame, J.; Kipper, K.; Koppel, I. A.; Koppel, I.; Zhang, M.; Mashima, M.; Yagupolskii, L. M.; Garlyauskayte, R. Y.; Filatov, A. A. J. Phys. Chem. A 2009, 113, 8421-8424; Zhdanko, A.; Maier, M. E. ACS Catal. 2015, 5, 59946004. ${ }^{b}{ }^{19} \mathrm{~F}$ NMR yield based on $\mathrm{PhCF}_{3} .{ }^{c}{ }^{1} \mathrm{H}$ NMR yield based on $\mathrm{CH}_{2} \mathrm{Br}_{2}$.

\section{Table S7.}

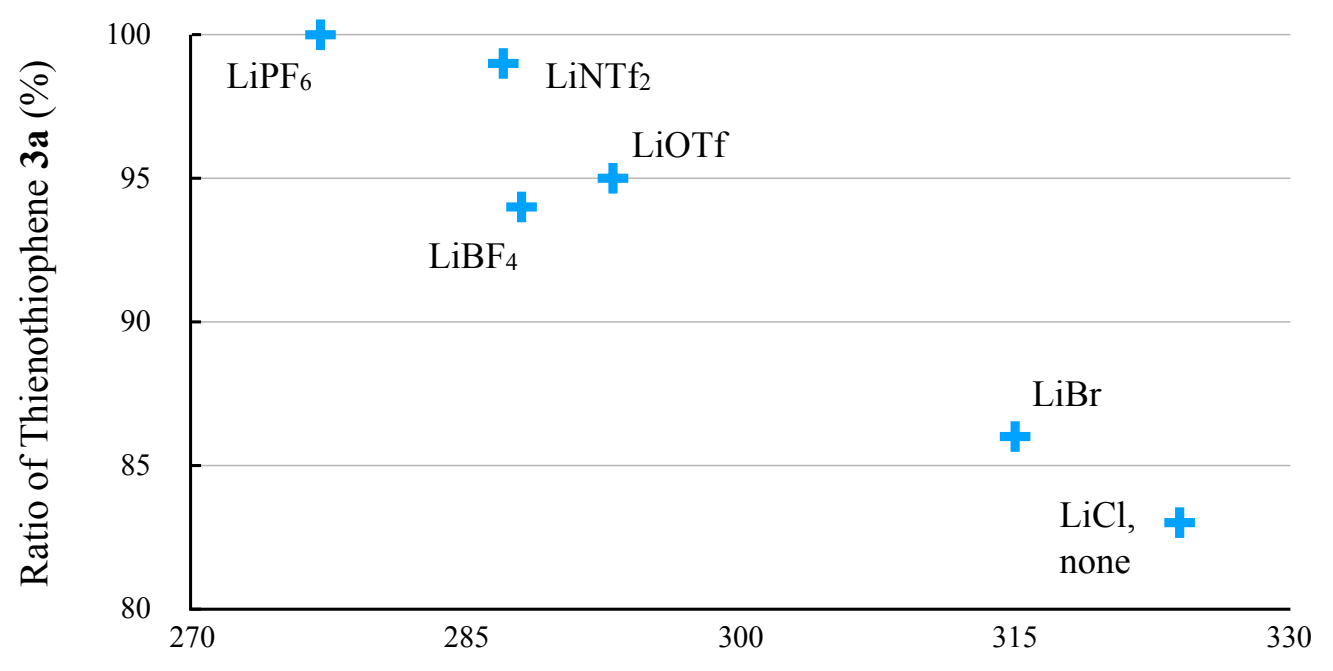

Gas Phase Acidity of HX / kcal mol-1 
6-2. Effect of Crown Ethers

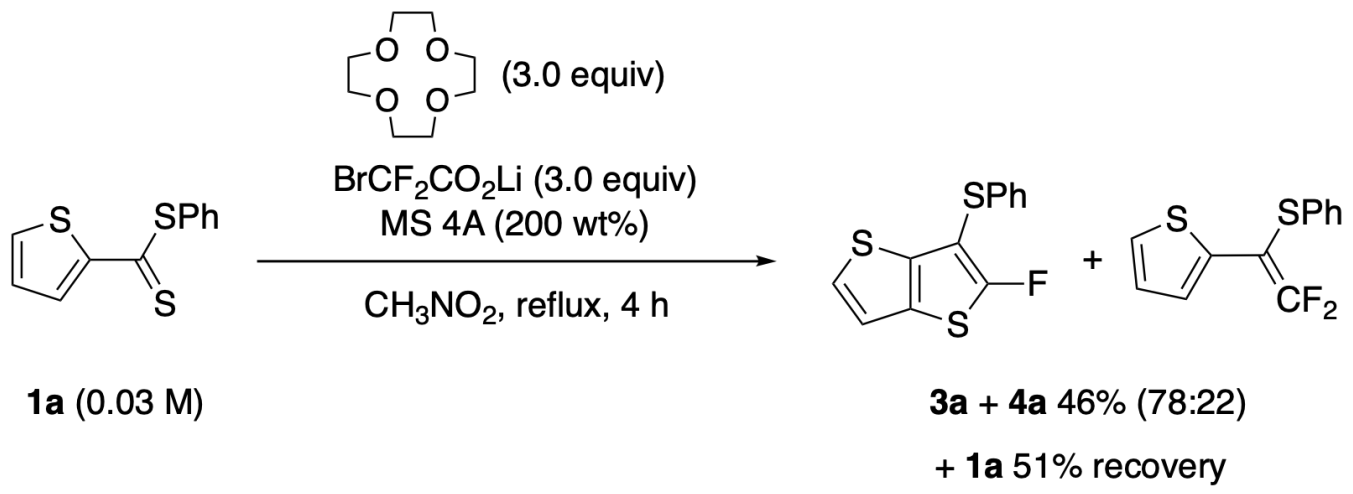

Scheme S2. 


\section{Typical Procedures}

\section{7-1. Synthesis of Sulfanylated Fluorothienothiophenes (Method A)}

Synthesis of 3a is described as a typical procedure. A two-necked flask containing MS 4A (141 mg, powder) was flame dried under vacuum. After cooling, thiophenecarbodithioate 1a (71 mg, $0.30 \mathrm{mmol})$, lithium bromodifluoroacetate $(163 \mathrm{mg}, 0.90 \mathrm{mmol})$, and nitromethane $(9.0$ $\mathrm{mL}$ ) was added. The resulting suspension was refluxed for $7 \mathrm{~h}$ (an oil bath). Phosphate buffer ( $\mathrm{pH}$ $=7$ ) was added to quench the reaction at room temperature. Organic materials were extracted with dichloromethane three times. The combined extracts were washed with brine and dried over anhydrous $\mathrm{Na}_{2} \mathrm{SO}_{4}$. After removal of the solvent under reduced pressure, the residue was purified by column chromatography on silica gel (hexane) to give thienothiophene $\mathbf{3 a}$ (65 mg, $81 \%$ yield) as colorless crystals.

\section{7-2. Synthesis of Sulfanylated Fluorothienothiophenes and -furans (Method B)}

Synthesis of $\mathbf{3 d}$ is described as a typical procedure. A test tube (microwave vessel) containing MS 4A (86 mg, powder) was flame dried under vacuum. After cooling, thiophenecarbodithioate $1 \mathbf{d}(86 \mathrm{mg}, 0.30 \mathrm{mmol})$, lithium bromodifluoroacetate $(163 \mathrm{mg}, 0.90$ $\mathrm{mmol})$, and nitromethane $(3.0 \mathrm{~mL})$ was added, and the mixture was irradiated with microwave $(90 \mathrm{~W})$ at $140{ }^{\circ} \mathrm{C}$ for $1 \mathrm{~h}$ (sealed). Phosphate buffer $(\mathrm{pH}=7)$ was added to quench the reaction at room temperature. Organic materials were extracted with dichloromethane three times. The combined extracts were washed with brine and dried over anhydrous $\mathrm{Na}_{2} \mathrm{SO}_{4}$. After removal of the solvent under reduced pressure, the residue was purified by column chromatography on silica gel (hexane) to give thienothiophene 3d (69 mg, 73\% yield) as colorless crystals.

\section{7-3. Synthesis of Pentacyclic Thienothienofurans}

Synthesis of $\mathbf{8 b}$ is described as a typical procedure. To a THF solution $(0.8 \mathrm{~mL})$ of fluorothienofuran $7 \mathbf{b}(52 \mathrm{mg}, 0.14 \mathrm{mmol})$ was added a pentane solution $(1.8 \mathrm{M}, 0.15 \mathrm{~mL})$ of tert-butyllithium $(0.27 \mathrm{mmol})$ at $-78{ }^{\circ} \mathrm{C}$. The resulting solution was slowly warmed to room temperature and stirred for 2 days. Phosphate buffer $(\mathrm{pH}=7)$ was added to quench the reaction at room temperature. Organic materials were extracted with chloroform three times. The combined extracts were washed with brine and dried over anhydrous $\mathrm{Na}_{2} \mathrm{SO}_{4}$. After removal of the solvent under reduced pressure, the residue was purified by column chromatography on silica gel (hexane/toluene 30:1) to give thienothienofuran $\mathbf{8 b}$ (26 mg, 67\% yield) as colorless crystals. 


\section{Spectral Data of Thiophene- and Furancarbodithioates}

8-1. Thiophenecarbodithioates $\mathbf{1}$

8-1-1. Phenyl thiophene-2-carbodithioate 1a

Red crystals (Mp. $36-37^{\circ} \mathrm{C}$ ), $2.10 \mathrm{~g}, 90 \%$ yield.

${ }^{1} \mathrm{H}$ NMR: $\delta=7.96(\mathrm{dd}, J=4.0,1.1 \mathrm{~Hz}, 1 \mathrm{H}), 7.67(\mathrm{dd}, J=5.1,1.1$

$\mathrm{Hz}, 1 \mathrm{H}), 7.52-7.48$ (m, 5H), $7.16(\mathrm{dd}, J=5.1,4.0 \mathrm{~Hz}, 1 \mathrm{H})$.

${ }^{13} \mathrm{C}$ NMR: $\delta=213.8,151.2,135.8,135.5,130.43,130.41,129.5$, 128.6, 127.1 .

IR (neat): $\tilde{v}=1500,1404,1350,1252,1049,989,773,717 \mathrm{~cm}^{-1}$.

HRMS (EI): $m / z$ calcd. for $\mathrm{C}_{11} \mathrm{H}_{8} \mathrm{~S}_{3}[\mathrm{M}]^{+}: 235.9788$; found: 235.9791

8-1-2. Phenyl 5-methylthiophene-2-carbodithioate 1b

Red crystals (Mp. $\left.73-74{ }^{\circ} \mathrm{C}\right), 2.77 \mathrm{~g}, 90 \%$ yield.

${ }^{1} \mathrm{H}$ NMR: $\delta=7.80(\mathrm{~d}, J=3.9 \mathrm{~Hz}, 1 \mathrm{H}), 7.48(\mathrm{~m}, 5 \mathrm{H}), 6.84(\mathrm{dq}, J$

$=3.9,0.9 \mathrm{~Hz}, 1 \mathrm{H}), 2.49(\mathrm{~s}, 3 \mathrm{H})$.

${ }^{13}$ C NMR: $\delta=212.7,152.3,149.1,135.9,130.5,130.3,129.4$, $127.7,127.5,16.2$.

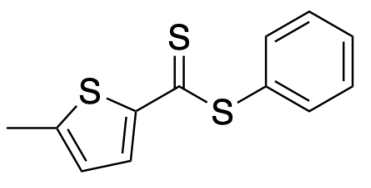

1b

IR (neat): $\tilde{v}=2910,1439,1338,1255,1163,1039,795,750 \mathrm{~cm}^{-1}$.

HRMS (EI): $m / z$ calcd. for $\mathrm{C}_{12} \mathrm{H}_{10} \mathrm{~S}_{3}[\mathrm{M}]^{+}:$249.9945; found: 249.9943.

8-1-3. Phenyl 5-phenylthiophene-2-carbodithioate 1c

Red crystals (Mp. $118-119^{\circ} \mathrm{C}$ ), 2.05 g, 85\% yield.

${ }^{1} \mathrm{H}$ NMR: $\delta=7.93(\mathrm{~d}, J=4.1 \mathrm{~Hz}, 1 \mathrm{H}), 7.67(\mathrm{dd}, J=8.1,1.6 \mathrm{~Hz}$, 2H), 7.52-7.48 (m, 5H), 7.42-7.35 (m, 4H).

${ }^{13} \mathrm{C}$ NMR: $\delta=212.4,154.8,149.8,135.8,133.2,130.4,129.5$,

129.2, 129.1, 128.1, 126.1, 124.5.

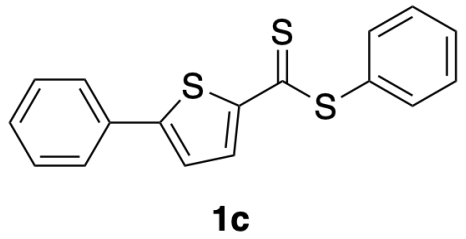

IR (neat): $\tilde{v}=1346,1261,1045,993,804,687 \mathrm{~cm}^{-1}$.

HRMS (EI): $m / z$ calcd. for $\mathrm{C}_{17} \mathrm{H}_{12} \mathrm{~S}_{3}[\mathrm{M}]^{+}:$312.0101; found: 312.0114 .

8-1-4. Phenyl benzothiophene-2-carbodithioate 1d

Red crystals (Mp. $142-143{ }^{\circ} \mathrm{C}$ ), 3.06 g, 59\% yield.

${ }^{1} \mathrm{H}$ NMR: $\delta=8.23(\mathrm{~s}, 1 \mathrm{H}), 7.92(\mathrm{~d}, J=8.0 \mathrm{~Hz}, 1 \mathrm{H}), 7.81(\mathrm{~d}, J=$ $8.0 \mathrm{~Hz}, 1 \mathrm{H}), 7.52(\mathrm{~m}, 5 \mathrm{H}), 7.46(\mathrm{dd}, J=8.0,8.0 \mathrm{~Hz}, 1 \mathrm{H}), 7.40$ $(\mathrm{dd}, J=8.0,8.0 \mathrm{~Hz}, 1 \mathrm{H})$.

${ }^{13} \mathrm{C}$ NMR: $\delta=214.9,150.0,143.7,139.6,135.7,130.5,130.3$,

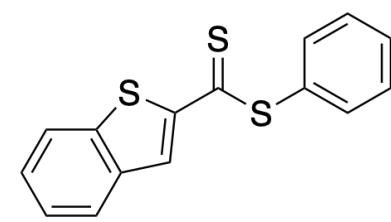

$1 d$ $129.6,127.4,126.5,125.3,123.4,122.4$. 
IR (neat): $\tilde{v}=1493,1335,1219,1176,1039,866,768,687 \mathrm{~cm}^{-1}$.

HRMS (EI): $m / z$ calcd. for $\mathrm{C}_{15} \mathrm{H}_{10} \mathrm{~S}_{3}[\mathrm{M}]^{+}$: 285.9945; found: 285.9944.

8-1-5. Phenyl 5-methylbenzothiophene-2-carbodithioate 1e

Red crystals (Mp. $142-142{ }^{\circ} \mathrm{C}$ ), 1.16 g, 88\% yield.

${ }^{1} \mathrm{H}$ NMR: $\delta=8.12(\mathrm{~s}, 1 \mathrm{H}), 7.67(\mathrm{~d}, J=8.4 \mathrm{~Hz}, 1 \mathrm{H}), 7.66(\mathrm{~s}, 1 \mathrm{H})$, 7.51-7.48 (m, 5H), $7.26(\mathrm{dd}, J=8.4,1.5 \mathrm{~Hz}, 1 \mathrm{H}), 2.45$ (s, 3H).

${ }^{13} \mathrm{C}$ NMR: $\delta=214.8,150.0,141.1,139.8,135.7,135.1,130.4$, $130.3,129.5,129.3,126.1,123.2,122.0,21.3$.

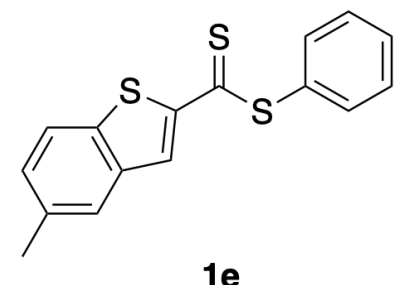

IR (neat): $\tilde{v}=1498,1201,1036,783,729,685 \mathrm{~cm}^{-1}$.

HRMS (EI): $m / z$ Calcd. for $\mathrm{C}_{16} \mathrm{H}_{12} \mathrm{~S}_{3}[\mathrm{M}]^{+}:$300.0101; found: 300.0113 .

8-1-6. Phenyl 5-chlorobenzothiophene-2-carbodithioate 1f

Red crystals (Mp. 109-110 ${ }^{\circ} \mathrm{C}$ ), $853 \mathrm{mg}, 76 \%$ yield.

${ }^{1} \mathrm{H}$ NMR: $\delta=8.12(\mathrm{~s}, 1 \mathrm{H}), 7.88(\mathrm{~d}, J=2.0 \mathrm{~Hz}, 1 \mathrm{H}), 7.73(\mathrm{~d}, J=$ $8.6 \mathrm{~Hz}, 1 \mathrm{H}), 7.55-7.49(\mathrm{~m}, 5 \mathrm{H}), 7.41(\mathrm{dd}, J=8.6,2.0 \mathrm{~Hz}, 1 \mathrm{H})$.

${ }^{13} \mathrm{C}$ NMR: $\delta=214.7,151.6,141.6,140.5,135.6,131.4,130.7$, $130.0,129.7,127.7,125.5,123.5,122.1$.

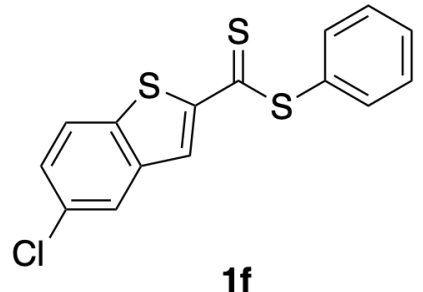

IR (neat): $\tilde{v}=1491,1174,1038,881,771 \mathrm{~cm}^{-1}$.

HRMS (EI): $m / z$ Calcd. for $\mathrm{C}_{15} \mathrm{H}_{9} \mathrm{ClS}_{3}[\mathrm{M}]^{+}: 319.9555$; found: 319.9546 .

8-1-7. 2-Bromophenyl benzothiophene-2-carbodithioate $\mathbf{1 g}$

Red crystals (Mp. 109-109 ${ }^{\circ} \mathrm{C}$ ), 4.71 g, 94\% yield.

${ }^{1} \mathrm{H}$ NMR: $\delta=8.21(\mathrm{~s}, 1 \mathrm{H}), 7.91(\mathrm{~d}, J=8.0 \mathrm{~Hz}, 1 \mathrm{H}), 7.80(\mathrm{br} \mathrm{d}$, $J=8.2 \mathrm{~Hz}, 1 \mathrm{H}), 7.77(\mathrm{dd}, J=8.0,1.4 \mathrm{~Hz}, 1 \mathrm{H}), 7.60(\mathrm{dd}, J=7.7$, $1.8 \mathrm{~Hz}, 1 \mathrm{H}), 7.45(\mathrm{dd}, J=7.3,1.2 \mathrm{~Hz}, 1 \mathrm{H}), 7.42$ (dd, $J=7.5,1.4$ $\mathrm{Hz}, 1 \mathrm{H}), 7.40-7.36(\mathrm{~m}, 2 \mathrm{H})$.

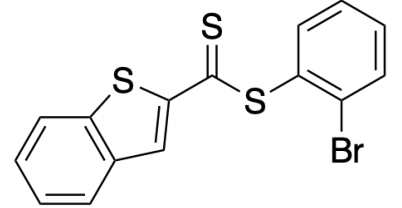

19

${ }^{13} \mathrm{C}$ NMR: $\delta=211.4,149.6,143.8,139.5,138.1,133.8,132.2,131.7,130.8,128.4,127.4$, $126.5,125.3,123.7,122.4$.

IR (neat): $\tilde{v}=1493,1219,1182,1043,771 \mathrm{~cm}^{-1}$.

EA: Calcd. for $\mathrm{C}_{15} \mathrm{H}_{9} \mathrm{BrS}_{3}$ : C, 49.32; H, 2.48\%, found: C, 49.12; H, 2.21\%.

8-1-8. Phenyl thiophene-3-carbodithioate $\mathbf{1 h}$

Red crystals, (Mp. $36-37^{\circ} \mathrm{C}$ ), $1.82 \mathrm{~g}, 86 \%$ yield.

${ }^{1} \mathrm{H}$ NMR: $\delta=8.27(\mathrm{dd}, J=3.1,1.4 \mathrm{~Hz}, 1 \mathrm{H}), 7.77(\mathrm{dd}, J=5.1,1.4$ $\mathrm{Hz}, 1 \mathrm{H}), 7.51-7.46$ (m, 5H), $7.31(\mathrm{dd}, J=5.1,3.1 \mathrm{~Hz}, 1 \mathrm{H})$.

${ }^{13} \mathrm{C}$ NMR: $\delta=217.8,147.5,135.6,130.6,130.3,129.5,127.6$,<smiles>S=C(Sc1ccccc1)c1ccsc1</smiles>
127.3, 126.0. 
IR (neat): $\tilde{v}=1496,1406,1196,1041,933,835,735 \mathrm{~cm}^{-1}$.

HRMS (EI): $m / z$ Calcd. for $\mathrm{C}_{11} \mathrm{H}_{8} \mathrm{~S}_{3}[\mathrm{M}]^{+}$: 235.9788; found: 235.9784 .

8-1-9. Phenyl 5-methylthiophene-3-carbodithioate 1i

Red crystals (Mp. $\left.39-40{ }^{\circ} \mathrm{C}\right), 1.05 \mathrm{~g}, 82 \%$ yield.

${ }^{1} \mathrm{H}$ NMR: $\delta=8.06(\mathrm{~d}, J=1.1 \mathrm{~Hz}, 1 \mathrm{H}), 7.51-7.45(\mathrm{~m}, 5 \mathrm{H}), 7.44$

$(\mathrm{dq}, J=1.1,1.1 \mathrm{~Hz}, 1 \mathrm{H}), 2.49(\mathrm{~d}, J=1.1 \mathrm{~Hz}, 3 \mathrm{H})$.

${ }^{13} \mathrm{C}$ NMR: $\delta=217.9,147.3,140.5,135.6,130.7,130.3,129.5$,

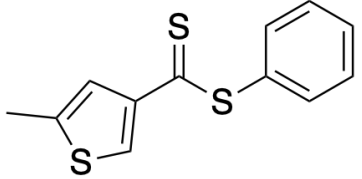

$1 \mathbf{i}$ $125.8,125.3,15.3$.

IR (neat): $\tilde{v}=3095,2918,1431,1194,1059,949,737,685 \mathrm{~cm}^{-1}$.

HRMS (EI): $m / z$ Calcd. for $\mathrm{C}_{12} \mathrm{H}_{10} \mathrm{~S}_{3}[\mathrm{M}]^{+}:$249.9945; found: 249.9949.

8-1-10.Phenyl 5-phenylthiophene-3-carbodithioate $\mathbf{1 j}$

Red crystals (Mp. 68-69 ${ }^{\circ} \mathrm{C}$ ), $812 \mathrm{mg}, 69 \%$ yield.

${ }^{1} \mathrm{H}$ NMR: $\delta=8.22(\mathrm{~d}, J=1.4 \mathrm{~Hz}, 1 \mathrm{H}), 7.96(\mathrm{~d}, J=1.4 \mathrm{~Hz}, 1 \mathrm{H})$, $7.63(\mathrm{~d}, J=7.4 \mathrm{~Hz}, 2 \mathrm{H}), 7.52-7.46(\mathrm{~m}, 5 \mathrm{H}), 7.41(\mathrm{dd}, J=7.4,7.4$ $\mathrm{Hz}, 2 \mathrm{H}), 7.33$ (t, $J=7.4 \mathrm{~Hz}, 1 \mathrm{H})$.

${ }^{13} \mathrm{C}$ NMR: $\delta=217.6,148.0,144.9,135.6,133.3,130.6,130.4$,<smiles>S=C(Sc1ccccc1)c1csc(-c2ccccc2)c1</smiles>

$1 \mathrm{j}$ $129.6,129.0,128.3,126.6,126.0,122.8$.

IR (neat): $\tilde{v}=1439,1203,1063,970,883,742,685 \mathrm{~cm}^{-1}$.

HRMS (EI): $m / z$ Calcd. for $\mathrm{C}_{17} \mathrm{H}_{12} \mathrm{~S}_{3}[\mathrm{M}]^{+}:$312.0101; found: 312.0105 .

8-1-11. Phenyl 5-bromothiophene-3-carbodithioate 1k

Red crystals (Mp. $\left.86-87^{\circ} \mathrm{C}\right), 1.24$ g, 77\% yield.

${ }^{1} \mathrm{H}$ NMR: $\delta=8.14(\mathrm{~d}, J=1.6 \mathrm{~Hz}, 1 \mathrm{H}), 7.73(\mathrm{~d}, J=1.6 \mathrm{~Hz}, 1 \mathrm{H})$, 7.54-7.48 (m, 3H), 7.47-7.44 (m, 2H).

${ }^{13} \mathrm{C}$ NMR: $\delta=216.3,147.2,135.5,130.5,130.2,129.8,129.6$, 128.1, 113.4 .

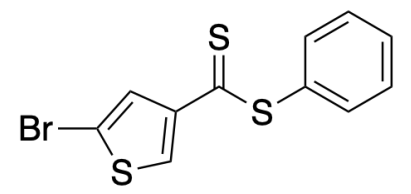

IR (neat): $\tilde{v}=1414,1198,1059,972,850,746,692 \mathrm{~cm}^{-1}$.

HRMS (EI): $m / z$ Calcd. for $\mathrm{C}_{11} \mathrm{H}_{7} \mathrm{BrS}_{3}[\mathrm{M}]^{+}:$313.8893; found: 313.8892 .

8-1-12.4-Methoxyphenyl thiophene-3-carbodithioate 11

Red crystals (Mp. $\left.111-112{ }^{\circ} \mathrm{C}\right), 616$ g, 84\% yield.

${ }^{1} \mathrm{H}$ NMR: $\delta=8.27(\mathrm{dd}, J=3.0,1.3 \mathrm{~Hz}, 1 \mathrm{H}), 7.77(\mathrm{dd}, J=5.1,1.3$

$\mathrm{Hz}, 1 \mathrm{H}), 7.45(\mathrm{~d}, J=8.9 \mathrm{~Hz}, 2 \mathrm{H}), 7.32(\mathrm{dd}, J=5.1,3.0 \mathrm{~Hz}, 1 \mathrm{H})$,

$7.02(\mathrm{~d}, J=8.9 \mathrm{~Hz}, 2 \mathrm{H}), 3.87$ (s, 3H).

${ }^{13} \mathrm{C}$ NMR: $\delta=219.1,161.3,147.4,137.0,127.5,127.3,126.0$,<smiles>COc1ccc(SC(=S)c2ccsc2)cc1</smiles>

11

$121.4,115.1,55.3$. 
IR (neat): $\tilde{v}=3093,2960,1589,1491,1398,1244,1022,802 \mathrm{~cm}^{-1}$.

HRMS (EI): $m / z$ Calcd. for $\mathrm{C}_{12} \mathrm{H}_{10} \mathrm{OS}_{3}[\mathrm{M}]^{+}:$265.9894; found: 265.9892 .

8-1-13.4-Chlorophenyl thiophene-3-carbodithioate $\mathbf{1 m}$

Red crystals (Mp. $\left.80-81{ }^{\circ} \mathrm{C}\right), 838 \mathrm{mg}, 77 \%$ yield.

${ }^{1} \mathrm{H}$ NMR: $\delta=8.27(\mathrm{~d}, J=3.1 \mathrm{~Hz}, 1 \mathrm{H}), 7.76(\mathrm{~d}, J=5.1 \mathrm{~Hz}, 1 \mathrm{H})$, 7.47 (d, $J=8.4 \mathrm{~Hz}, 2 \mathrm{H}), 7.39$ (d, $J=8.4 \mathrm{~Hz}, 2 \mathrm{H}), 7.32$ (dd, $J=$ 5.1, 3.1 Hz, 1H).

${ }^{13} \mathrm{C}$ NMR: $\delta=216.9,147.3,136.9,136.9,129.9,128.9,127.8$, 127.2, 126.2 .

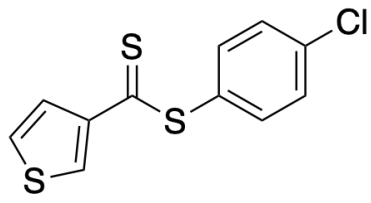

$1 \mathrm{~m}$

IR (neat): $\tilde{v}=1473,1248,1200,1039,930,785,638 \mathrm{~cm}^{-1}$.

HRMS (EI): $m / z$ Calcd. for $\mathrm{C}_{11} \mathrm{H}_{7} \mathrm{ClS}_{3}[\mathrm{M}]^{+}:$269.9398; found: 269.9395.

8-1-14. Phenyl benzothiophene-3-carbodithioate 1n

Red crystals (Mp. 90-91 ${ }^{\circ} \mathrm{C}$ ), 1.13 g, 88\% yield.

${ }^{1} \mathrm{H}$ NMR: $\delta=8.60(\mathrm{~d}, J=7.8 \mathrm{~Hz}, 1 \mathrm{H}), 8.40(\mathrm{~s}, 1 \mathrm{H}), 7.85(\mathrm{~d}, J$ $=7.8 \mathrm{~Hz}, 1 \mathrm{H}), 7.54-7.49(\mathrm{~m}, 5 \mathrm{H}), 7.45(\mathrm{td}, J=7.8,1.2 \mathrm{~Hz}, 1 \mathrm{H})$, $7.39(\mathrm{td}, J=7.8,1.2 \mathrm{~Hz}, 1 \mathrm{H})$.

${ }^{13} \mathrm{C}$ NMR: $\delta=218.8,141.8,140.5,136.3,135.5,130.6,130.4$,<smiles>S=C(Sc1ccccc1)c1csc2ccccc12</smiles>
$130.2,129.6,125.5,124.8,122.5$.

IR (neat): $\tilde{v}=1454,1411,1201,1012,997,729,685 \mathrm{~cm}^{-1}$.

HRMS (EI): $m / z$ calcd. for $\mathrm{C}_{15} \mathrm{H}_{10} \mathrm{~S}_{3}[\mathrm{M}]^{+}: 285.9945$; found: 285.9949 .

8-1-15.2-Bromophenyl benzothiophene-3-carbodithioate 10

Red crystals (Mp. $88-89^{\circ} \mathrm{C}$ ), 1.64 g, 51\% yield.

${ }^{1} \mathrm{H}$ NMR: $\delta=8.63(\mathrm{~d}, J=7.4 \mathrm{~Hz}, 1 \mathrm{H}), 8.47(\mathrm{~s}, 1 \mathrm{H}), 7.88(\mathrm{~d}, J=$

$7.4 \mathrm{~Hz}, 1 \mathrm{H}), 7.80(\mathrm{dd}, J=7.4,1.1 \mathrm{~Hz}, 1 \mathrm{H}), 7.62(\mathrm{dd}, J=7.4,1.6$

$\mathrm{Hz}, 1 \mathrm{H}), 7.46$ (br dd, $J=7.4,7.4 \mathrm{~Hz}, 2 \mathrm{H}), 7.41$ (dd, $J=7.4,7.4$

$\mathrm{Hz}, 1 \mathrm{H}), 7.40$ (ddd, $J=7.4,7.4,1.6 \mathrm{~Hz}, 1 \mathrm{H})$.

${ }^{13} \mathrm{C}$ NMR: $\delta=215.4,141.6,140.6,137.9,136.3,133.9,132.1$,<smiles>S=C(Sc1ccccc1Br)c1csc2ccccc12</smiles>

$130.8,130.6,128.5,125.59,125.57,124.8,122.6$.

IR (neat): $\tilde{v}=1412,1203,1009,746 \mathrm{~cm}^{-1}$.

EA: Calcd. for $\mathrm{C}_{15} \mathrm{H}_{9} \mathrm{BrS}_{3}$ : C, 49.32; H, 2.48\%, found: C, 49.05; H, 2.37\%.

8-2. Furancarbodithioates 6

8-2-1. Phenyl benzofuran-2-carbodithioate 6a

Red crystals (Mp. $78-79{ }^{\circ} \mathrm{C}$ ), $4.3 \mathrm{~g}, 80 \%$ yield.

${ }^{1} \mathrm{H}$ NMR: $\delta=7.71(\mathrm{~d}, J=0.9 \mathrm{~Hz}, 1 \mathrm{H}), 7.66($ br d, $J=7.9 \mathrm{~Hz}$,

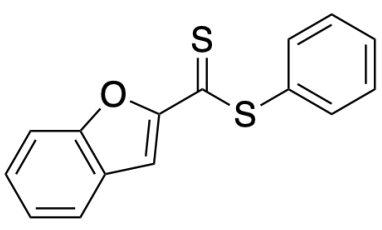

$6 a$ 
1H), $7.55(\mathrm{dd}, J=7.9,0.7 \mathrm{~Hz}, 1 \mathrm{H}), 7.50-7.43(\mathrm{~m}, 6 \mathrm{H}), 7.26(\mathrm{ddd}, J=7.9,7.9,0.9 \mathrm{~Hz}, 1 \mathrm{H})$.

${ }^{13} \mathrm{C}$ NMR: $\delta=208.6,157.1,155.9,135.8,130.5,129.6,129.2,128.5,127.8,124.1,123.2$, 112.4, 111.3.

IR (neat): $\tilde{v}=1529,1281,1130,1047,739 \mathrm{~cm}^{-1}$.

HRMS (EI): $m / z$ Calcd. for $\mathrm{C}_{15} \mathrm{H}_{10} \mathrm{OS}_{2}[\mathrm{M}]^{+}:$270.0173; found: 270.0180 .

8-2-2. 2-Bromophenyl benzofuran-2-carbodithioate $\mathbf{6 b}$

Red crystals (Mp. $114-116{ }^{\circ} \mathrm{C}$ ), 8.6 g, 82\% yield.

${ }^{1} \mathrm{H}$ NMR: $\delta=7.79(\mathrm{dd}, J=7.8,1.4 \mathrm{~Hz}, 1 \mathrm{H}), 7.79(\mathrm{~d}, J=1.0 \mathrm{~Hz}$, 1H), 7.73 (br d, $J=7.8 \mathrm{~Hz}, 1 \mathrm{H}), 7.62-7.58$ (m, 2H), 7.52 (ddd, $J$ $=7.2,7.2,1.2 \mathrm{~Hz}, 1 \mathrm{H}), 7.46(\mathrm{ddd}, J=7.8,7.8,1.8 \mathrm{~Hz}, 1 \mathrm{H}), 7.40$ (ddd, $J=7.8,7.8,1.8 \mathrm{~Hz}, 1 \mathrm{H}), 7.32$ (ddd, $J=7.2,7.2,0.9 \mathrm{~Hz}$, $1 \mathrm{H})$.

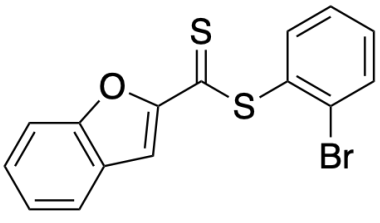

6b

${ }^{13} \mathrm{C}$ NMR: $\delta=205.1,157.0,155.9,138.1,133.9,132.2,131.0,130.8,128.7,128.5,127.9$, $124.2,123.2,112.5,111.7$.

IR (neat): $\tilde{v}=1529,1284,1057,741 \mathrm{~cm}^{-1}$.

EA: Calcd. for $\mathrm{C}_{15} \mathrm{H}_{9} \mathrm{BrOS}_{2}:$ C, 51.58; H, 2.60\%, found: C, 51.48; H, 2.38\%. 


\section{Spectral Data of Thienothiophenes, Difluoroalkenes, Thienofurans, and Thienothienofurans}

\section{9-1. Thienothiophenes 3}

9-1-1. 2-Fluoro-3-(phenylsulfanyl)thieno[3,2-b]thiophene 3a

Colorless crystals (Mp. $\left.<30{ }^{\circ} \mathrm{C}\right), 65 \mathrm{mg}, 81 \%$ yield.

${ }^{1} \mathrm{H}$ NMR: $\delta=7.32-7.24(\mathrm{~m}, 5 \mathrm{H}), 7.21-7.16(\mathrm{~m}, 2 \mathrm{H})$.

${ }^{13} \mathrm{C}$ NMR: $\delta=165.3\left(\mathrm{~d}, J_{\mathrm{CF}}=297 \mathrm{~Hz}\right), 137.3,134.0,129.1,128.9$,

$126.7,125.1\left(\mathrm{~d}, J_{\mathrm{CF}}=5 \mathrm{~Hz}\right), 124.1\left(\mathrm{~d}, J_{\mathrm{CF}}=3 \mathrm{~Hz}\right), 119.8,103.9(\mathrm{~d}$,

$\left.J_{\mathrm{CF}}=14 \mathrm{~Hz}\right)$.

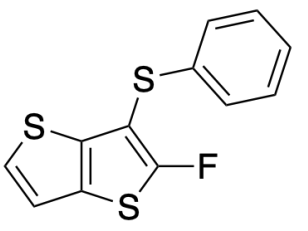

${ }^{19}$ F NMR: $\delta=47.0(\mathrm{~s})$.

IR (neat): $\tilde{v}=1523,1479,1348,1219,1084,1024,941,771 \mathrm{~cm}^{-1}$.

HRMS (EI): $m / z$ calcd. for $\mathrm{C}_{12} \mathrm{H}_{7} \mathrm{FS}_{3}[\mathrm{M}]^{+}:$265.9694; found: 265.9687.

9-1-2. 2-Fluoro-5-methyl-3-(phenylsulfanyl)thieno[3,2-b]thiophene 3b

Colorless crystals (Mp. $40-41{ }^{\circ} \mathrm{C}$ ), $67 \mathrm{mg}, 80 \%$ yield.

${ }^{1} \mathrm{H}$ NMR: $\delta=7.28-7.21(\mathrm{~m}, 4 \mathrm{H}), 7.17(\mathrm{tt}, J=6.8,1.7 \mathrm{~Hz}, 1 \mathrm{H})$,

$6.80(\mathrm{q}, J=1.2 \mathrm{~Hz}, 1 \mathrm{H}), 2.50(\mathrm{~s}, 3 \mathrm{H})$.

${ }^{13} \mathrm{C}$ NMR: $\delta=164.4\left(\mathrm{~d}, J_{\mathrm{CF}}=296 \mathrm{~Hz}\right), 140.2\left(\mathrm{~d}, J_{\mathrm{CF}}=5 \mathrm{~Hz}\right)$,

$135.3,134.2,129.0,128.7,126.6,123.6\left(\mathrm{~d}, J_{\mathrm{CF}}=4 \mathrm{~Hz}\right), 117.5$,

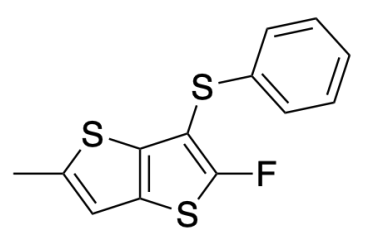

$103.6\left(\mathrm{~d}, J_{\mathrm{CF}}=14 \mathrm{~Hz}\right), 16.0$.

${ }^{19}$ F NMR: $\delta=43.4(\mathrm{~s})$.

IR (neat): $\tilde{v}=2918,1581,1512,1477,1207,935,737,688 \mathrm{~cm}^{-1}$.

HRMS (EI): $m / z$ Calcd. for $\mathrm{C}_{13} \mathrm{H}_{9} \mathrm{FS}_{3}[\mathrm{M}]^{+}:$279.9850; found: 279.9851.

9-1-3. 2-Fluoro-5-phenyl-3-(phenylsulfanyl)thieno[3,2-b]thiophene 3c

Colorless crystals (Mp. 102-103 ${ }^{\circ} \mathrm{C}$ ), $67 \mathrm{mg}, 65 \%$ yield.

${ }^{1} \mathrm{H}$ NMR: $\delta=7.53-7.51(\mathrm{~m}, 2 \mathrm{H}), 7.37-7.33(\mathrm{~m}, 3 \mathrm{H}), 7.32-2.29$

(m, 2H), 7.29-7.24 (m, 3H), 7.19 (tt, $J=7.1,1.4 \mathrm{~Hz}, 1 \mathrm{H})$.

${ }^{13} \mathrm{C}$ NMR: $\delta=165.1\left(\mathrm{~d}, J_{\mathrm{CF}}=297 \mathrm{~Hz}\right), 144.4\left(\mathrm{~d}, J_{\mathrm{CF}}=5 \mathrm{~Hz}\right)$,

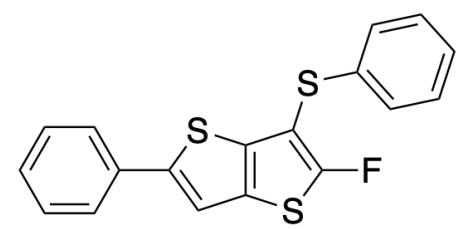

$136.7,134.0,133.9,129.1,129.0,128.8,127.9,126.8,125.7,125.1$

$\left(\mathrm{d}, J_{\mathrm{CF}}=4 \mathrm{~Hz}\right), 115.6,104.0$.

3c

${ }^{19}$ F NMR: $\delta=46.0(\mathrm{~s})$.

IR (neat): $\tilde{v}=1518,1477,1441,1207,737,687 \mathrm{~cm}^{-1}$.

HRMS (EI): $m / z$ calcd. for $\mathrm{C}_{18} \mathrm{H}_{11} \mathrm{FS}_{3}[\mathrm{M}]^{+}:$342.0007; found: 342.0006 . 
9-1-4. 2-Fluoro-3-(phenylsulfanyl)thieno[3,2-b][f]benzothiophene 3d

Colorless crystals (Mp. 94-95 ${ }^{\circ} \mathrm{C}$ ), $69 \mathrm{mg}, 73 \%$ yield.

${ }^{1} \mathrm{H}$ NMR: $\delta=7.79(\mathrm{~d}, J=8.0 \mathrm{~Hz}, 1 \mathrm{H}), 7.71(\mathrm{~d}, J=8.0 \mathrm{~Hz}, 1 \mathrm{H})$, $7.40(\mathrm{dd}, J=8.0,8.0 \mathrm{~Hz}, 1 \mathrm{H}), 7.33-7.30$ (m, 3H), 7.25 (t, $J=7.4$ $\mathrm{Hz}, 2 \mathrm{H}), 7.20(\mathrm{t}, J=7.4 \mathrm{~Hz}, 1 \mathrm{H})$.

${ }^{13} \mathrm{C}$ NMR: $\delta=165.7\left(\mathrm{~d}, J_{\mathrm{CF}}=299 \mathrm{~Hz}\right), 139.9\left(\mathrm{~d}, J_{\mathrm{CF}}=3 \mathrm{~Hz}\right)$, $137.1,134.0,133.1,129.2,128.9,126.8,125.0,124.3,123.6$,

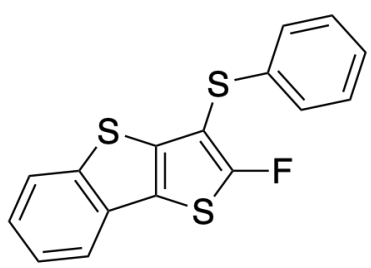

3d $120.0,119.4\left(\mathrm{~d}, J_{\mathrm{CF}}=4 \mathrm{~Hz}\right), 105.1\left(\mathrm{~d}, J_{\mathrm{CF}}=14 \mathrm{~Hz}\right)$.

${ }^{19}$ F NMR: $\delta=48.2$ (s).

IR (neat): $\tilde{v}=1508,1475,1331,1207,1022,928,829,735 \mathrm{~cm}^{-1}$. HRMS (EI): $m / z$ Calcd. for $\mathrm{C}_{16} \mathrm{H}_{9} \mathrm{FS}_{3}[\mathrm{M}]^{+}: 315.9850$; found: 315.9850 .

9-1-5. 2-Fluoro-7-methyl-3-(phenylsulfanyl)benzo[b]thieno[2,3-d] thiophene 3e

Colorless crystals (Mp. 143-143 ${ }^{\circ} \mathrm{C}$ ), 70 mg, 78\% yield.

${ }^{1} \mathrm{H}$ NMR: $\delta=7.64(\mathrm{~d}, J=8.2 \mathrm{~Hz}, 1 \mathrm{H}), 7.50(\mathrm{~s}, 1 \mathrm{H}), 7.29(\mathrm{~d}, J=$ $7.6 \mathrm{~Hz}, 2 \mathrm{H}), 7.24$ (dd, $J=7.3,7.3 \mathrm{~Hz}, 2 \mathrm{H}), 7.18$ (t, $J=7.3 \mathrm{~Hz}$, $1 \mathrm{H}), 7.12(\mathrm{~d}, J=8.2 \mathrm{~Hz}, 1 \mathrm{H}), 2.46(\mathrm{~s}, 3 \mathrm{H})$.

${ }^{13} \mathrm{C}$ NMR: $\delta=165.6\left(\mathrm{~d}, J_{\mathrm{CF}}=298 \mathrm{~Hz}\right), 137.2,136.9\left(\mathrm{~d}, J_{\mathrm{CF}}=3\right.$ Hz), 134.9, 134.0, 133.3, 129.1, 128.8, 126.8, 125.9, 123.2, 120.1,

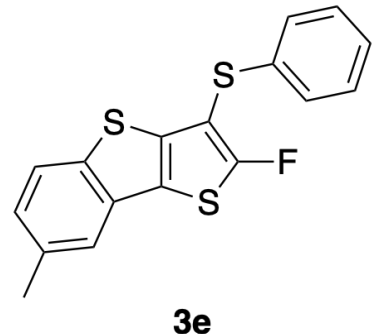
$119.1\left(\mathrm{~d}, J_{\mathrm{CF}}=4 \mathrm{~Hz}\right), 104.9\left(\mathrm{~d}, J_{\mathrm{CF}}=14 \mathrm{~Hz}\right), 21.4$.

${ }^{19}$ F NMR: $\delta=47.9(\mathrm{~s})$.

IR (neat): $\tilde{v}=2916,1477,1441,1209,1024,804 \mathrm{~cm}^{-1}$.

HRMS (EI): $m / z$ calcd. for $\mathrm{C}_{17} \mathrm{H}_{11} \mathrm{FS}_{3}[\mathrm{M}]^{+}: 330.0007$; found: 330.0008 .

9-1-6. 7-Chloro-2-fluoro-3-(phenylsulfanyl)benzo[b]thieno[2,3-d] thiophene 3f

Colorless crystals, $58 \mathrm{mg}, 55 \%$ yield.

${ }^{1} \mathrm{H}$ NMR: $\delta=7.71-7.68(\mathrm{~m}, 2 \mathrm{H}), 7.33-7.30(\mathrm{~m}, 2 \mathrm{H}), 7.28-7.25$ (m, 3H), 7.23-7.19 (m, 1H).

${ }^{13} \mathrm{C}$ NMR: $\delta=166.0\left(\mathrm{~d}, J_{\mathrm{CF}}=300 \mathrm{~Hz}\right), 138.8,138.0\left(\mathrm{~d}, J_{\mathrm{CF}}=3\right.$ $\mathrm{Hz}), 134.3,133.6\left(\mathrm{~d}, J_{\mathrm{CF}}=2 \mathrm{~Hz}\right), 131.3,129.2,129.1,127.1,124.6$ $\left(\mathrm{d}, J_{\mathrm{CF}}=1 \mathrm{~Hz}\right), 124.5,119.7,118.6\left(\mathrm{~d}, J_{\mathrm{CF}}=4 \mathrm{~Hz}\right), 105.4\left(\mathrm{~d}, J_{\mathrm{CF}}=\right.$

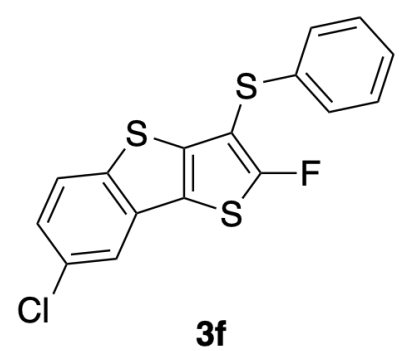
$14 \mathrm{~Hz})$.

${ }^{19}$ F NMR: $\delta=49.5$ (s).

IR (neat): $\tilde{v}=1516,1439,1205,1076,997,802,737,687 \mathrm{~cm}^{-1}$. HRMS (EI): $m / z$ calcd. for $\mathrm{C}_{16} \mathrm{H}_{8} \mathrm{ClFS}_{3}[\mathrm{M}]^{+}: 349.9461$; found: 349.9453 .

9-1-7. 3-[(2-Bromophenyl)sulfanyl]-2-fluorobenzo[b]thieno[2,3-d]thiophene $\mathbf{3 g}$ Colorless crystals (Mp. $\left.117-118{ }^{\circ} \mathrm{C}\right), 35 \mathrm{mg}, 79 \%$ yield. 
${ }^{1} \mathrm{H}$ NMR: $\delta=7.82(\mathrm{brd}, J=7.8 \mathrm{~Hz}, 1 \mathrm{H}), 7.76(\mathrm{brd}, J=7.8 \mathrm{~Hz}$, 1H), $7.56(\mathrm{dd}, J=7.9,1.3 \mathrm{~Hz}, 1 \mathrm{H}), 7.43(\mathrm{ddd}, J=7.8,7.8,1.1$ $\mathrm{Hz}, 1 \mathrm{H}), 7.35$ (ddd, $J=7.8,7.8,1.1 \mathrm{~Hz}, 1 \mathrm{H}), 7.11$ (ddd, $J=7.8$, 7.8, $1.4 \mathrm{~Hz}, 1 \mathrm{H}), 7.03$ (ddd, $J=7.9,7.9,1.6 \mathrm{~Hz}, 1 \mathrm{H}), 6.89$ (dd, $J$ $=7.9,1.4 \mathrm{~Hz}, 1 \mathrm{H})$.

${ }^{13} \mathrm{C}$ NMR: $\delta=166.8\left(\mathrm{~d}, J_{\mathrm{CF}}=300 \mathrm{~Hz}\right), 139.8,136.9,135.6,133.1$,

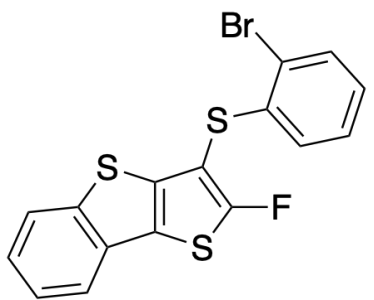

$3 g$ $133.0,127.9,127.7,127.3,125.0,124.4,123.6,121.6,120.0,119.8\left(\mathrm{~d}, J_{\mathrm{CF}}=4 \mathrm{~Hz}\right), 103.4(\mathrm{~d}$, $\left.J_{\mathrm{CF}}=14 \mathrm{~Hz}\right)$.

${ }^{19}$ F NMR: $\delta=50.6(\mathrm{~s})$.

IR (neat): $\tilde{v}=1518,1444,1335,1209,1018,930,742 \mathrm{~cm}^{-1}$.

HRMS (EI): $m / z$ calcd. for $\mathrm{C}_{16} \mathrm{H}_{8} \mathrm{BrFS}_{3}[\mathrm{M}]^{+}$: 395.8935 ; found: 395.8937 .

9-1-8. 2-Fluoro-3-(phenylsulfanyl)thieno[2,3-b]thiophene $\mathbf{3 h}$

Colorless crystals (Mp. $46-47^{\circ} \mathrm{C}$ ), $65 \mathrm{mg}, 81 \%$ yield.

${ }^{1} \mathrm{H}$ NMR: $\delta=7.28\left(\mathrm{dd}, J=5.3 \mathrm{~Hz}, J_{\mathrm{HF}}=2.5 \mathrm{~Hz}, 1 \mathrm{H}\right), 7.23-7.19$ (m, 4H), 7.16-7.12 (m, 1H), $7.03(\mathrm{~d}, J=5.3 \mathrm{~Hz}, 1 \mathrm{H})$.

${ }^{13} \mathrm{C}$ NMR: $\delta=165.6\left(\mathrm{~d}, J_{\mathrm{CF}}=296 \mathrm{~Hz}\right), 141.9,135.4,129.0,127.7$, $126.8,126.2,123.1\left(\mathrm{~d}, J_{\mathrm{CF}}=6 \mathrm{~Hz}\right), 120.5\left(\mathrm{~d}, J_{\mathrm{CF}}=4 \mathrm{~Hz}\right), 103.0$

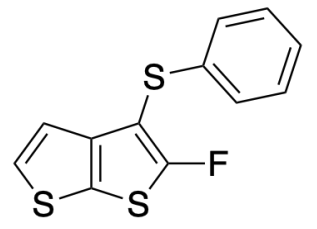

3h $\left(\mathrm{d}, J_{\mathrm{CF}}=11 \mathrm{~Hz}\right)$.

${ }^{19}$ F NMR: $\delta=48.5\left(\mathrm{~d}, J_{\mathrm{FH}}=2 \mathrm{~Hz}\right)$.

IR (neat): $\tilde{v}=1581,1539,1477,1198,1082,858,735,685 \mathrm{~cm}^{-1}$.

HRMS (EI): $m / z$ calcd. for $\mathrm{C}_{12} \mathrm{H}_{7} \mathrm{FS}_{3}[\mathrm{M}]^{+}: 265.9694$; found: 265.9698 .

9-1-9. 2-Fluoro-5-methyl-3-(phenylsulfanyl)thieno[2,3-b]thiophene 3i

Colorless crystals (Mp. $52-53{ }^{\circ} \mathrm{C}$ ), $60 \mathrm{mg}, 71 \%$ yield.

${ }^{1} \mathrm{H}$ NMR: $\delta=7.23(\mathrm{dd}, J=7.6,7.6 \mathrm{~Hz}, 2 \mathrm{H}), 7.18(\mathrm{dd}, J=7.6,1.5$ $\mathrm{Hz}, 2 \mathrm{H}), 7.14$ (tt, $J=7.6,1.5 \mathrm{~Hz}, 1 \mathrm{H}), 6.72$ (q, $J=1.2 \mathrm{~Hz}, 1 \mathrm{H})$, $2.49(\mathrm{~d}, J=1.2 \mathrm{~Hz}, 3 \mathrm{H})$.

${ }^{13}$ C NMR: $\delta=165.5\left(\mathrm{~d}, J_{\mathrm{CF}}=296 \mathrm{~Hz}\right), 141.9,141.8,135.7\left(\mathrm{~d}, J_{\mathrm{CF}}\right.$ $=2 \mathrm{~Hz}), 129.0,127.5,126.0,120.5\left(\mathrm{~d}, J_{\mathrm{CF}}=6 \mathrm{~Hz}\right), 118.5\left(\mathrm{~d}, J_{\mathrm{CF}}\right.$

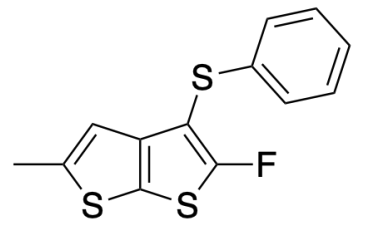

3i $=3 \mathrm{~Hz}), 102.5\left(\mathrm{~d}, J_{\mathrm{CF}}=11 \mathrm{~Hz}\right), 15.9$.

${ }^{19}$ F NMR: $\delta=46.2$ (s).

IR (neat): $\tilde{v}=2912,1579,1519,1477,1200,1068,737,688 \mathrm{~cm}^{-1}$. HRMS (EI): $m / z$ Calcd. for $\mathrm{C}_{13} \mathrm{H}_{9} \mathrm{FS}_{3}[\mathrm{M}]^{+}:$279.9850; found: 279.9851.

9-1-10.2-Fluoro-5-phenyl-3-(phenylsulfanyl)thieno[2,3-b]thiophene 3j

Colorless crystals (Mp. 79-80 ${ }^{\circ} \mathrm{C}$ ), $80 \mathrm{mg}, 78 \%$ yield.

${ }^{1} \mathrm{H}$ NMR: $\delta=7.52(\mathrm{~d}, J=7.3 \mathrm{~Hz}, 2 \mathrm{H}), 7.35(\mathrm{dd}, J=7.3,7.3 \mathrm{~Hz}, 2 \mathrm{H}), 7.28(\mathrm{t}, J=7.3 \mathrm{~Hz}$, $1 \mathrm{H}), 7.24-7.23(\mathrm{~m}, 5 \mathrm{H}), 7.13-7.17(\mathrm{~m}, 1 \mathrm{H})$. 
${ }^{13} \mathrm{C}$ NMR: $\delta=165.7\left(\mathrm{~d}, J_{\mathrm{CF}}=297 \mathrm{~Hz}\right), 146.3,142.7,135.4\left(\mathrm{~d}, J_{\mathrm{CF}}\right.$ $=2 \mathrm{~Hz}), 133.9,129.1,129.0,128.0,127.6,126.2,125.9,122.5(\mathrm{~d}$, $\left.J_{\mathrm{CF}}=6 \mathrm{~Hz}\right), 116.3\left(\mathrm{~d}, J_{\mathrm{CF}}=4 \mathrm{~Hz}\right), 103.0\left(\mathrm{~d}, J_{\mathrm{CF}}=11 \mathrm{~Hz}\right)$.

${ }^{19}$ F NMR: $\delta=47.6(\mathrm{~s})$.

IR (neat): $\tilde{v}=1545,1477,1439,1192,1072,756,688 \mathrm{~cm}^{-1}$.

HRMS (EI): $\mathrm{m} / z$ Calcd. for $\mathrm{C}_{18} \mathrm{H}_{11} \mathrm{FS}_{3}[\mathrm{M}]^{+}:$342.0007; found:

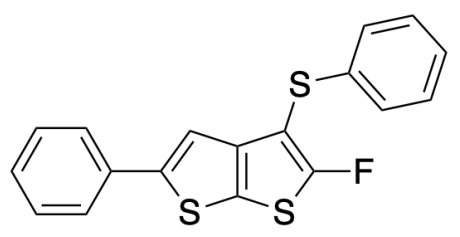

342.0008 .

9-1-11.5-Bromo-2-fluoro-3-(phenylsulfanyl)thieno[2,3-b]thiophene 3k

Colorless crystals (Mp. $76-77{ }^{\circ} \mathrm{C}$ ), $72 \mathrm{mg}, 69 \%$ yield.

${ }^{1} \mathrm{H}$ NMR: $\delta=7.26(\mathrm{t}, J=6.2 \mathrm{~Hz}, 2 \mathrm{H}), 7.20-7.16(\mathrm{~m}, 3 \mathrm{H}), 7.04$ (s, $1 \mathrm{H})$.

${ }^{13}$ C NMR: $\delta=166.1\left(\mathrm{~d}, J_{\mathrm{CF}}=297 \mathrm{~Hz}\right), 141.7\left(\mathrm{~d}, J_{\mathrm{CF}}=2 \mathrm{~Hz}\right)$, $135.0,129.2,127.8,126.4,123.5\left(\mathrm{~d}, J_{\mathrm{CF}}=4 \mathrm{~Hz}\right), 122.8\left(\mathrm{~d}, J_{\mathrm{CF}}=\right.$ $7 \mathrm{~Hz}), 112.8,103.2\left(\mathrm{~d}, J_{\mathrm{CF}}=12 \mathrm{~Hz}\right)$.

3j

${ }^{19}$ F NMR: $\delta=46.4(\mathrm{~s})$.

IR (neat): $\tilde{v}=1541,1477,1439,1219,1186,1066,737,688 \mathrm{~cm}^{-1}$.

HRMS (EI): $m / z$ Calcd. for $\mathrm{C}_{12} \mathrm{H}_{6} \mathrm{BrFS}_{3}[\mathrm{M}]^{+}: 343.8799$; found: 343.8800 .

9-1-12.2-Fluoro-3-[(4-methoxyphenyl)sulfanyl] thieno[2,3-b]thiophene 31

Colorless crystals (Mp. 57-58 ${ }^{\circ} \mathrm{C}$ ), $64 \mathrm{mg}, 72 \%$ yield.

${ }^{1} \mathrm{H}$ NMR: $\delta=7.28(\mathrm{~d}, J=8.9 \mathrm{~Hz}, 2 \mathrm{H}), 7.28-7.26(\mathrm{~m}, 1 \mathrm{H}), 7.04$

(d, $J=5.3 \mathrm{~Hz}, 1 \mathrm{H}), 6.79$ (d, $J=8.9 \mathrm{~Hz}, 2 \mathrm{H}), 3.75$ (s, 3H).

${ }^{13} \mathrm{C}$ NMR: $\delta=164.4\left(\mathrm{~d}, J_{\mathrm{CF}}=295 \mathrm{~Hz}\right), 159.0,141.8,131.5$, $126.6,125.3\left(\mathrm{~d}, J_{\mathrm{CF}}=1 \mathrm{~Hz}\right), 123.0\left(\mathrm{~d}, J_{\mathrm{CF}}=6 \mathrm{~Hz}\right), 120.5\left(\mathrm{~d}, J_{\mathrm{CF}}\right.$ $=4 \mathrm{~Hz}), 114.7,105.0\left(\mathrm{~d}, J_{\mathrm{CF}}=11 \mathrm{~Hz}\right), 55.3$.

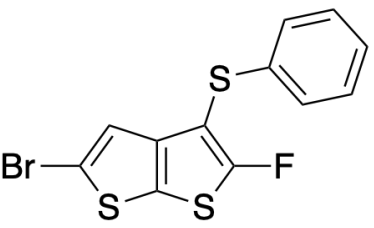

3k

${ }^{19}$ F NMR: $\delta=46.9(\mathrm{~s})$.

IR (neat): $\tilde{v}=2835,1541,1489,1242,1198,1028,822,719 \mathrm{~cm}^{-1}$.

HRMS (EI): $m / z$ Calcd. for $\mathrm{C}_{13} \mathrm{H}_{9} \mathrm{FOS}_{3}[\mathrm{M}]^{+}:$295.9800; found: 295.9800 .

9-1-13.2-Fluoro-3-[(4-chlorophenyl)sulfanyl]thieno[2,3-b]thiophene 3m

Colorless crystals (Mp. 60-61 ${ }^{\circ} \mathrm{C}$ ), $54 \mathrm{mg}, 60 \%$ yield .

${ }^{1} \mathrm{H}$ NMR: $\delta=7.33\left(\mathrm{dd}, J=5.3 \mathrm{~Hz}, J_{\mathrm{HF}}=2.5 \mathrm{~Hz}, 1 \mathrm{H}\right), 7.20(\mathrm{~d}, J$ $=8.6 \mathrm{~Hz}, 2 \mathrm{H}), 7.13(\mathrm{~d}, J=8.6 \mathrm{~Hz}, 2 \mathrm{H}), 7.03(\mathrm{~d}, J=5.3 \mathrm{~Hz}, 1 \mathrm{H})$.

${ }^{13} \mathrm{C}$ NMR: $\delta=165.8\left(\mathrm{~d}, J_{\mathrm{CF}}=297 \mathrm{~Hz}\right), 141.6,133.9\left(\mathrm{~d}, J_{\mathrm{CF}}=2\right.$ $\mathrm{Hz}), 132.2,129.2,129.0,127.0,123.3\left(\mathrm{~d}, J_{\mathrm{CF}}=6 \mathrm{~Hz}\right), 120.3(\mathrm{~d}$, $\left.J_{\mathrm{CF}}=4 \mathrm{~Hz}\right), 102.6\left(\mathrm{~d}, J_{\mathrm{CF}}=11 \mathrm{~Hz}\right)$.

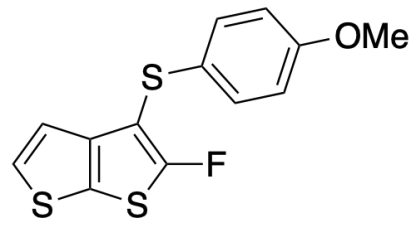

31

${ }^{19}$ F NMR: $\delta=48.9\left(\mathrm{~d}, J_{\mathrm{FH}}=2 \mathrm{~Hz}\right)$.

IR (neat): $\tilde{v}=1539,1473,1385,1203,1092,1011,812,719 \mathrm{~cm}^{-1}$. 
HRMS (EI): $m / z$ Calcd. for $\mathrm{C}_{12} \mathrm{H}_{6} \mathrm{ClFS}_{3}[\mathrm{M}]^{+}:$299.9304; found: 299.9305.

9-1-14.2-Fluoro-3-(phenylsulfanyl)benzo[b]thieno[3,2- $d]$ thiophene 3n

Colorless crystals (Mp. 94-95 ${ }^{\circ} \mathrm{C}$ ), $69 \mathrm{mg}, 73 \%$ yield.

${ }^{1} \mathrm{H}$ NMR: $\delta=8.34-8.32(\mathrm{~m}, 1 \mathrm{H}), 7.83-7.81(\mathrm{~m}, 1 \mathrm{H}), 7.33(\mathrm{ddd}$, $J=7.2,7.2,1.4 \mathrm{~Hz}, 2 \mathrm{H}), 7.22-7.20$ (m, 4H), 7.14-7.10 (m, 1H).

${ }^{13} \mathrm{C}$ NMR: $\delta=166.1\left(\mathrm{~d}, J_{\mathrm{CF}}=296 \mathrm{~Hz}\right), 141.7,136.0,134.9$, $133.50,133.48,129.2,126.8,126.0,124.69,124.65,122.7,121.9$,

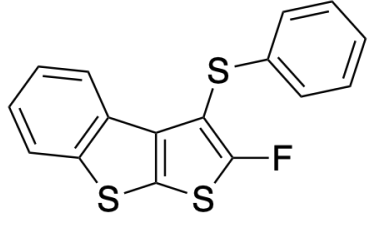

$3 n$

$104.1\left(\mathrm{~d}, J_{\mathrm{CF}}=12 \mathrm{~Hz}\right)$.

${ }^{19}$ F NMR: $\delta=48.1$ (s).

IR (neat): $\tilde{v}=1527,1477,1398,1215,910,739 \mathrm{~cm}^{-1}$.

HRMS (EI): $m / z$ Calcd. for $\mathrm{C}_{16} \mathrm{H}_{9} \mathrm{FS}_{3}[\mathrm{M}]^{+}:$315.9850; found: 315.9844 .

9-1-15.2-Fluoro-3-(2-bromophenylsulfanyl)benzo[b]thieno[3,2- $d]$ thiophene 30

Colorless crystals (Mp. $123-125^{\circ} \mathrm{C}$ ), $30 \mathrm{mg}, 76 \%$ yield.

${ }^{1} \mathrm{H}$ NMR: $\delta=8.17(\mathrm{br} \mathrm{d}, J=8.1 \mathrm{~Hz}, 1 \mathrm{H}), 7.82(\mathrm{~d}, J=8.1 \mathrm{~Hz}$, $1 \mathrm{H}), 7.56(\mathrm{dd}, J=8.1,1.5 \mathrm{~Hz}, 1 \mathrm{H}), 7.33(\mathrm{ddd}, J=8.1,8.1,1.0$ $\mathrm{Hz}, 1 \mathrm{H}), 7.30$ (br dd, $J=8.1,8.1 \mathrm{~Hz}, 1 \mathrm{H}), 7.03$ (ddd, $J=8.1,8.1$, $1.1 \mathrm{~Hz}, 1 \mathrm{H}), 6.97(\mathrm{ddd}, J=8.1,8.1,1.3 \mathrm{~Hz}, 1 \mathrm{H}), 6.75(\mathrm{dd}, J=$ $8.1,1.5 \mathrm{~Hz}, 1 \mathrm{H})$.

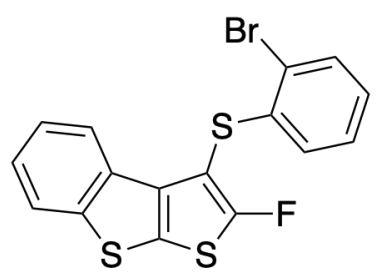

30

${ }^{13} \mathrm{C}$ NMR: $\delta=166.7\left(\mathrm{~d}, J_{\mathrm{CF}}=298 \mathrm{~Hz}\right), 141.8,137.3\left(\mathrm{~d}, J_{\mathrm{CF}}=2 \mathrm{~Hz}\right), 134.5,133.3\left(\mathrm{~d}, J_{\mathrm{CF}}=3\right.$ $\mathrm{Hz}), 133.0,128.1,126.7,126.5,124.9\left(\mathrm{~d}, J_{\mathrm{CF}}=8 \mathrm{~Hz}\right), 122.7,121.8,120.2,103.0\left(\mathrm{~d}, J_{\mathrm{CF}}=11\right.$ $\mathrm{Hz})$.

${ }^{19}$ F NMR: $\delta=49.7(\mathrm{~s})$.

IR (neat): $\tilde{v}=1527,1446,1217,1018,744 \mathrm{~cm}^{-1}$.

HRMS (EI): $m / z$ Calcd. for $\mathrm{C}_{16} \mathrm{H}_{8} \mathrm{BrFS}_{3}[\mathrm{M}]^{+}$: 393.8956; found: 393.8936 .

\section{9-2. Difluoroalkenes 4}

9-2-1. 1,1-Difluoro-2-(phenylsulfanyl)-2-(2-thienyl)ethene 4a

A colorless liquid, $61 \mathrm{mg}, 47 \%$ yield.

${ }^{1} \mathrm{H}$ NMR: $\delta=7.29(\mathrm{~d}, J=8.2 \mathrm{~Hz}, 2 \mathrm{H}), 7.26-7.22(\mathrm{~m}, 4 \mathrm{H}), 7.16(\mathrm{t}$, $J=7.4 \mathrm{~Hz}, 1 \mathrm{H}), 6.97(\mathrm{dd}, J=4.4 \mathrm{~Hz}, 1 \mathrm{H})$.

${ }^{13} \mathrm{C}$ NMR: $\delta=156.9\left(\mathrm{dd}, J_{\mathrm{CF}}=309,291 \mathrm{~Hz}\right), 136.0\left(\mathrm{~d}, J_{\mathrm{CF}}=8\right.$

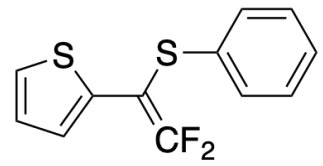

$\mathrm{Hz}), 134.4\left(\mathrm{dd}, J_{\mathrm{CF}}=2,2 \mathrm{~Hz}\right), 129.1,128.0,127.4,127.0\left(\mathrm{dd}, J_{\mathrm{CF}}\right.$

$=6,6 \mathrm{~Hz}), 126.6,126.3\left(\mathrm{dd}, J_{\mathrm{CF}}=6,3 \mathrm{~Hz}\right), 85.1\left(\mathrm{dd}, J_{\mathrm{CF}}=26,21 \mathrm{~Hz}\right)$.

${ }^{19}$ F NMR: $\delta=86.2(\mathrm{~d}, J=6 \mathrm{~Hz}, 1 \mathrm{~F}), 91.0(\mathrm{~d}, J=6 \mathrm{~Hz}, 1 \mathrm{~F})$.

IR (neat): $\tilde{v}=1678,1479,1281,1234,1201,1007,737,687 \mathrm{~cm}^{-1}$.

HRMS (EI): $m / z$ calcd. for $\mathrm{C}_{12} \mathrm{H}_{8} \mathrm{~F}_{2} \mathrm{~S}_{2}[\mathrm{M}]^{+}:$254.0036; found: 254.0039 . 


\section{9-3. Thienofurans 7}

9-3-1. 2-Fluoro-3-(phenylsulfanyl)thieno[3,2-b]benzofuran 7a

Colorless crystals (Mp. 84-85 ${ }^{\circ} \mathrm{C}$ ), $22 \mathrm{mg}, 72 \%$ yield.

${ }^{1} \mathrm{H}$ NMR: $\delta=7.61-7.55(\mathrm{~m}, 2 \mathrm{H}), 7.33-7.29$ (m, 4H), 7.25 (dd, $J$ $=7.3,7.3 \mathrm{~Hz}, 2 \mathrm{H}), 7.18(\mathrm{tt}, J=7.3,1.3 \mathrm{~Hz}, 1 \mathrm{H})$.

${ }^{13} \mathrm{C}$ NMR: $\delta=167.1\left(\mathrm{~d}, J_{\mathrm{CF}}=298 \mathrm{~Hz}\right), 156.7\left(\mathrm{~d}, J_{\mathrm{CF}}=3 \mathrm{~Hz}\right)$, $152.6\left(\mathrm{~d}, J_{\mathrm{CF}}=5 \mathrm{~Hz}\right), 134.7\left(\mathrm{~d}, J_{\mathrm{CF}}=2 \mathrm{~Hz}\right), 129.2,128.3,126.7$, 124.2, 123.6, 123.4, 118.4, 112.5, 104.9 (d, $\left.J_{\mathrm{CF}}=4 \mathrm{~Hz}\right), 98.3$ (d,

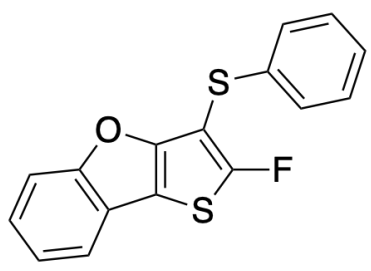

$7 a$ $J_{\mathrm{CF}}=15 \mathrm{~Hz}$ ).

${ }^{19}$ F NMR: $\delta=48.6(\mathrm{~s})$.

IR (neat): $\tilde{v}=1489,1442,1259,1217,1065,737 \mathrm{~cm}^{-1}$.

HRMS (EI): $m / z$ calcd. for $\mathrm{C}_{16} \mathrm{H}_{9} \mathrm{FOS}_{2}[\mathrm{M}]^{+}: 300.0079$; found: 300.0083 .

9-3-2. 2-Fluoro-3-[(2-bromophenyl)sulfanyl)]thieno[3,2-b]benzofuran 7b

Colorless crystals (Mp. 97-98 ${ }^{\circ} \mathrm{C}$ ), $24 \mathrm{mg}, 62 \%$ yield.

${ }^{1} \mathrm{H}$ NMR: $\delta=7.64-7.62(\mathrm{~m}, 1 \mathrm{H}), 7.58-7.55(\mathrm{~m}, 2 \mathrm{H}), 7.36-7.31$ $(\mathrm{m}, 2 \mathrm{H}), 7.12(\mathrm{dd}, J=7.9,7.9 \mathrm{~Hz}, 1 \mathrm{H}), 7.02(\mathrm{dd}, J=7.9,7.9 \mathrm{~Hz}$, $1 \mathrm{H}), 6.85(\mathrm{~d}, J=7.9 \mathrm{~Hz}, 1 \mathrm{H})$.

${ }^{13} \mathrm{C}$ NMR: $\delta=167.9\left(\mathrm{~d}, J_{\mathrm{CF}}=301 \mathrm{~Hz}\right), 156.8\left(\mathrm{~d}, J_{\mathrm{CF}}=3 \mathrm{~Hz}\right)$, $152.3\left(\mathrm{~d}, J_{\mathrm{CF}}=5 \mathrm{~Hz}\right), 136.3\left(\mathrm{~d}, J_{\mathrm{CF}}=2 \mathrm{~Hz}\right), 133.1,128.0,127.1$, $124.5,123.7,123.3,121.0,118.5,112.6,111.2,105.3\left(\mathrm{~d}, J_{\mathrm{CF}}=4\right.$

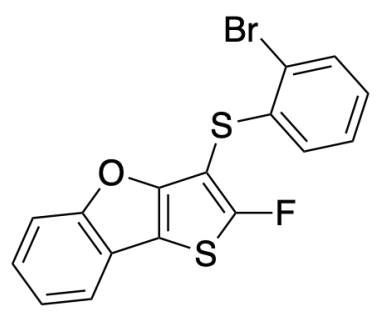

$7 b$

$\mathrm{Hz}), 96.7\left(\mathrm{~d}, J_{\mathrm{CF}}=15 \mathrm{~Hz}\right)$.

${ }^{19}$ F NMR: $\delta=50.6(\mathrm{~s})$.

IR (neat): $\tilde{v}=1489,1442,1217,1065,739 \mathrm{~cm}^{-1}$.

HRMS (EI): $m / z$ calcd. for $\mathrm{C}_{16} \mathrm{H}_{8} \mathrm{BrFOS}_{2}[\mathrm{M}]^{+}$: 377.9184 ; found: 377.9195 .

\section{9-4. Thienothienofurans 8}

9-4-1. Benzo[4',5'] thieno[2',3':4,5] thieno[3,2-b]benzofuran $\mathbf{8 b}$

Colorless crystals (Mp. 205-206 ${ }^{\circ} \mathrm{C}$ ), $26 \mathrm{mg}, 67 \%$ yield.

${ }^{1} \mathrm{H}$ NMR: $\delta=7.92-7.88(\mathrm{~m}, 2 \mathrm{H}), 7.75-7.71(\mathrm{~m}, 1 \mathrm{H}), 7.66-7.62$

$(\mathrm{m}, 1 \mathrm{H}), 7.46(\mathrm{dd}, J=7.6,7.6 \mathrm{~Hz}, 1 \mathrm{H}), 7.42-7.36(\mathrm{~m}, 3 \mathrm{H})$.

${ }^{13} \mathrm{C}$ NMR: $\delta=158.7,151.0,141.6,136.7,133.5,125.0,124.9$,

$124.8,124.5,124.1,123.5,121.5,121.2,120.4,119.0,112.6$.

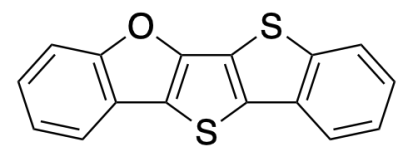

$8 b$

IR (neat): $\tilde{v}=1444,1378,1306,1263,738 \mathrm{~cm}^{-1}$.

HRMS (EI): $m / z$ calcd. for $\mathrm{C}_{16} \mathrm{H}_{8} \mathrm{OS}_{2}[\mathrm{M}]^{+}:$280.0016; found: 280.0029 . 


\section{ORTEP Diagrams of Products}

CCDC 2070421 (3d, Figure S5), 2070420 (3k, Figure S6), and 2070415 (3n, Figure S7) contain the supplementary crystallographic data. These data can be obtained free of charge from The Cambridge Crystallographic Data Centre via www.ccdc.cam.ac.uk/data_request/ cif.

\begin{tabular}{|c|c|c|c|}
\hline Identification code & mi004 & $\rho_{\text {calc }} \mathrm{g} / \mathrm{cm}^{3}$ & 1.575 \\
\hline Empirical formula & $\mathrm{C}_{16} \mathrm{H}_{9} \mathrm{FS}_{3}$ & $\mu / \mathrm{mm}^{-1}$ & 0.551 \\
\hline Formula weight & 316.41 & $\mathrm{~F}(000)$ & 648 \\
\hline Temperature/K & $120(2)$ & Crystal size $/ \mathrm{mm}^{3}$ & $0.65 \times 0.26 \times 0.25$ \\
\hline Crystal system & monoclinic & Radiation & $\operatorname{MoK} \alpha(\lambda=0.71073)$ \\
\hline Space group & $\mathrm{P} 2{ }_{1} / \mathrm{c}$ & $2 \theta$ range for data collection ${ }^{\circ}$ & 5.16 to 55.10 \\
\hline $\mathrm{a} / \AA$ & $14.1839(12)$ & Index ranges & $-18 \leq \mathrm{h} \leq 10,-7 \leq \mathrm{k} \leq 7,-20 \leq 1 \leq 20$ \\
\hline $\mathrm{b} / \AA$ & $5.9642(5)$ & Reflections collected & 3052 \\
\hline$c / \AA$ & $15.8359(13)$ & Independent reflections & 2753 \\
\hline$\alpha /{ }^{\circ}$ & 90.00 & & {$\left[\mathrm{R}_{\text {int }}=0.0165, \mathrm{R}_{\text {sigma }}=0.0199\right]$} \\
\hline$\beta /{ }^{\circ}$ & $94.8859(10)$ & Data/restraints/parameters & $3052 / 0 / 182$ \\
\hline$\gamma^{\circ}$ & 90.00 & Goodness-of-fit on $\mathrm{F}^{2}$ & 1.041 \\
\hline Volume $/ \AA^{3}$ & $1334.78(19)$ & Final $R$ indexes $[I \geq 2 \sigma(I)]$ & $\mathrm{R}_{1}=0.0259, \mathrm{wR}_{2}=0.0661$ \\
\hline Z & 4 & Final $\mathrm{R}$ indexes [all data] & $\mathrm{R}_{1}=0.0292, \mathrm{wR}_{2}=0.0687$ \\
\hline
\end{tabular}

Table S8.

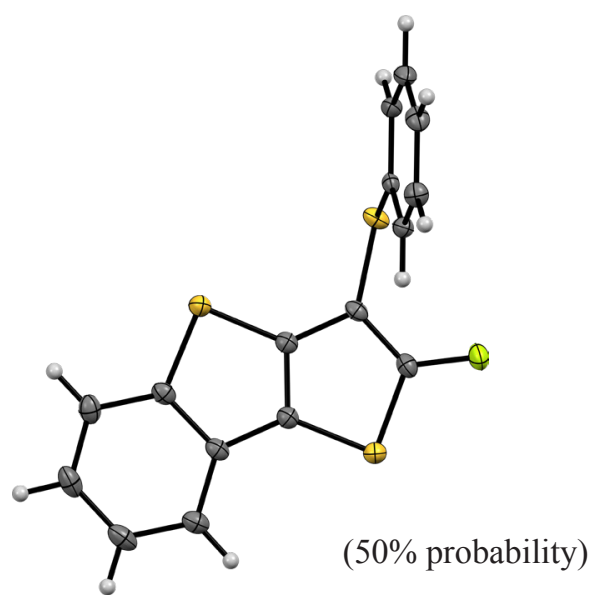

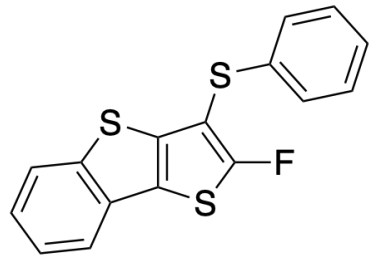

3d

Figure S5. 
Crystal data and structural refinement for $\mathbf{3 k}$

\begin{tabular}{|c|c|c|c|}
\hline Identification code & mi002 & $\rho_{\text {calc }} \mathrm{g} / \mathrm{cm}^{3}$ & 1.855 \\
\hline Empirical formula & $\mathrm{C}_{12} \mathrm{H}_{6} \mathrm{BrFS}_{3}$ & $\mu / \mathrm{mm}^{-1}$ & 3.814 \\
\hline Formula weight & 345.26 & $F(000)$ & 680 \\
\hline Temperature/K & $120(2)$ & Crystal size $/ \mathrm{mm}^{3}$ & $0.20 \times 0.07 \times 0.03$ \\
\hline Crystal system & monoclinic & Radiation & $\operatorname{MoK} \alpha(\lambda=0.71073)$ \\
\hline Space group & $\mathrm{P} 2 / 1 / n$ & $2 \theta$ range for data collection $/{ }^{\circ}$ & 6.34 to 54.78 \\
\hline $\mathrm{a} / \AA$ & $6.509(2)$ & Index ranges & $-7 \leq \mathrm{h} \leq 5,-30 \leq \mathrm{k} \leq 29,-9 \leq 1 \leq 8$ \\
\hline $\mathrm{b} / \AA$ & $25.547(9)$ & Reflections collected & 2170 \\
\hline$c / \AA$ & $7.603(3)$ & Independent reflections & 1747 \\
\hline$\alpha / 0$ & 90.00 & & {$\left[\mathrm{R}_{\text {int }}=0.0565, \mathrm{R}_{\text {sigma }}=0.0663\right]$} \\
\hline$\beta /{ }^{\circ}$ & $102.090(6)$ & Data/restraints/parameters & $2170 / 0 / 178$ \\
\hline$\gamma^{\prime 0}$ & 90.00 & Goodness-of-fit on $\mathrm{F}^{2}$ & 1.013 \\
\hline Volume $/ \AA^{3}$ & $1236.3(8)$ & Final $R$ indexes $[I \geq 2 \sigma(I)]$ & $\mathrm{R}_{1}=0.0365, \mathrm{wR}_{2}=0.0743$ \\
\hline Z & 4 & Final R indexes [all data] & $\mathrm{R}_{1}=0.0503, \mathrm{wR}_{2}=0.0797$ \\
\hline
\end{tabular}

Table S9.

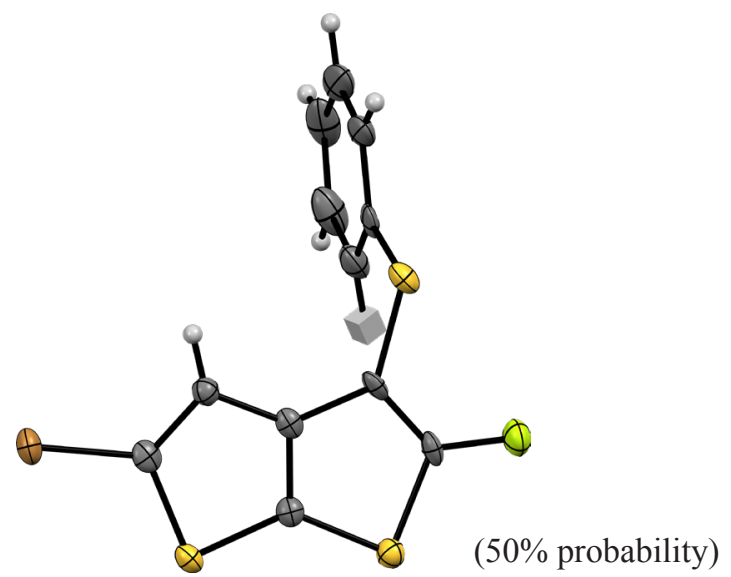

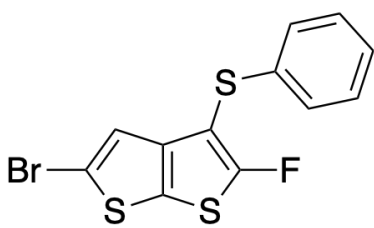

3k

Figure S6. 
Crystal data and structural refinement for $\mathbf{3 n}$

\begin{tabular}{|c|c|c|c|}
\hline Identification code & mi001 & $\rho_{\text {calc }} \mathrm{g} / \mathrm{cm}^{3}$ & 1.578 \\
\hline Empirical formula & $\mathrm{C}_{16} \mathrm{H}_{9} \mathrm{FS}_{3}$ & $\mu / \mathrm{mm}^{-1}$ & 0.552 \\
\hline Formula weight & 316.41 & $F(000)$ & 648 \\
\hline Temperature/K & $120(2)$ & Crystal size $/ \mathrm{mm}^{3}$ & $0.33 \times 0.06 \times 0.02$ \\
\hline Crystal system & monoclinic & Radiation & $\operatorname{MoK} \alpha(\lambda=0.71073)$ \\
\hline Space group & $\mathrm{P} 2 / 1 / n$ & $2 \theta$ range for data collection $/{ }^{\circ}$ & 5.56 to 54.68 \\
\hline $\mathrm{a} / \AA$ & $5.9331(11)$ & Index ranges & $-7 \leq \mathrm{h} \leq 7,-23 \leq \mathrm{k} \leq 16,-15 \leq 1 \leq 15$ \\
\hline $\mathrm{b} / \AA$ & $18.241(4)$ & Reflections collected & 3028 \\
\hline $\mathrm{c} / \AA$ & $12.343(2)$ & Independent reflections & 2515 \\
\hline$\alpha / 0$ & 90.00 & & {$\left[\mathrm{R}_{\text {int }}=0.0295, \mathrm{R}_{\text {sigma }}=0.0376\right]$} \\
\hline$\beta /{ }^{\circ}$ & $94.497(3)$ & Data/restraints/parameters & $3028 / 0 / 217$ \\
\hline$\gamma^{/ 0}$ & 90.00 & Goodness-of-fit on $\mathrm{F}^{2}$ & 1.056 \\
\hline Volume $/ \AA^{3}$ & $1331.7(4)$ & Final $R$ indexes $[I \geq 2 \sigma(I)]$ & $\mathrm{R}_{1}=0.0317, \mathrm{wR}_{2}=0.0717$ \\
\hline Z & 4 & Final R indexes [all data] & $\mathrm{R}_{1}=0.0412, \mathrm{wR}_{2}=0.0767$ \\
\hline
\end{tabular}

Table S10.

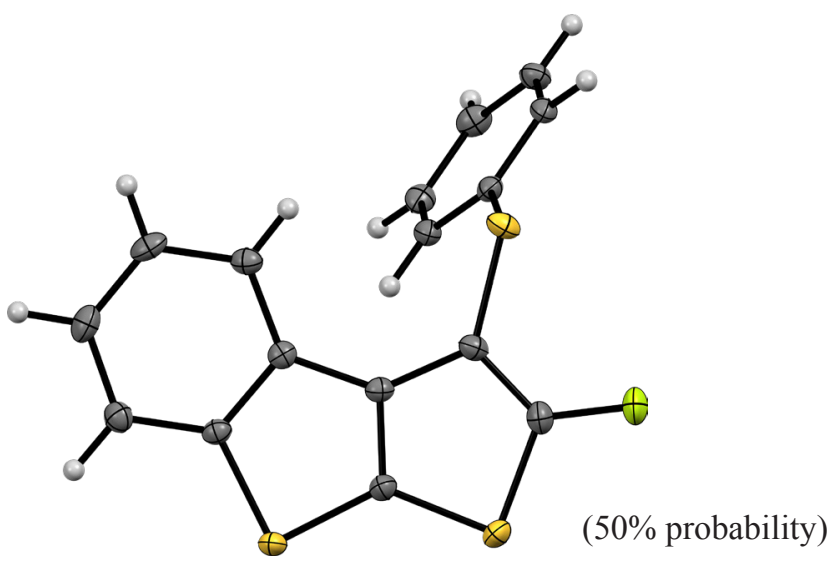

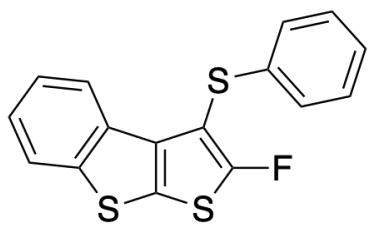

$3 n$

Figure S7. 


\section{NMR Spectra of Substrates and Products}

${ }^{1} \mathrm{H}$ NMR Spectrum of Phenyl thiophene-2-carbodithioate 1a

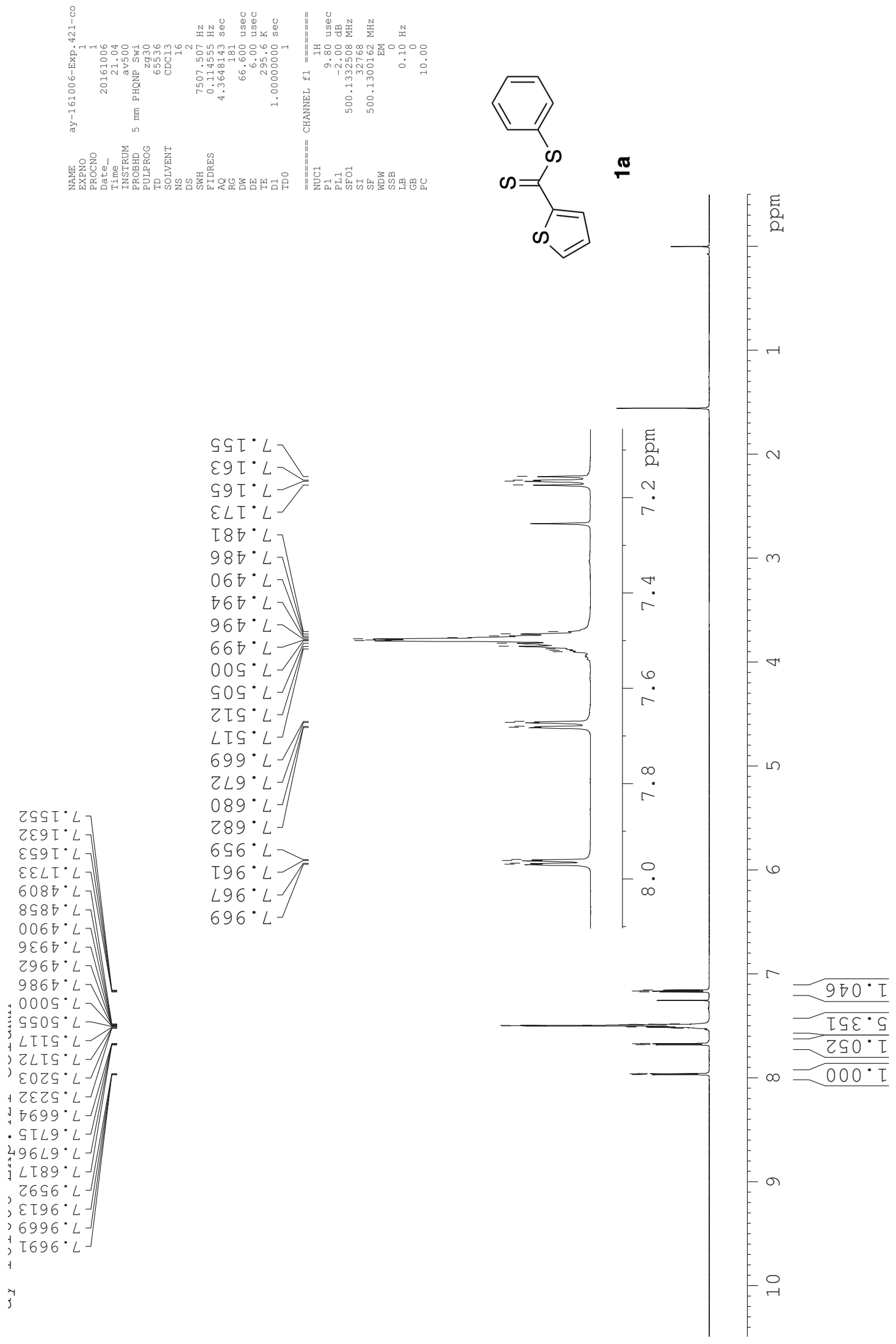


${ }^{13} \mathrm{C}$ NMR Spectrum of Phenyl thiophene-2-carbodithioate 1a
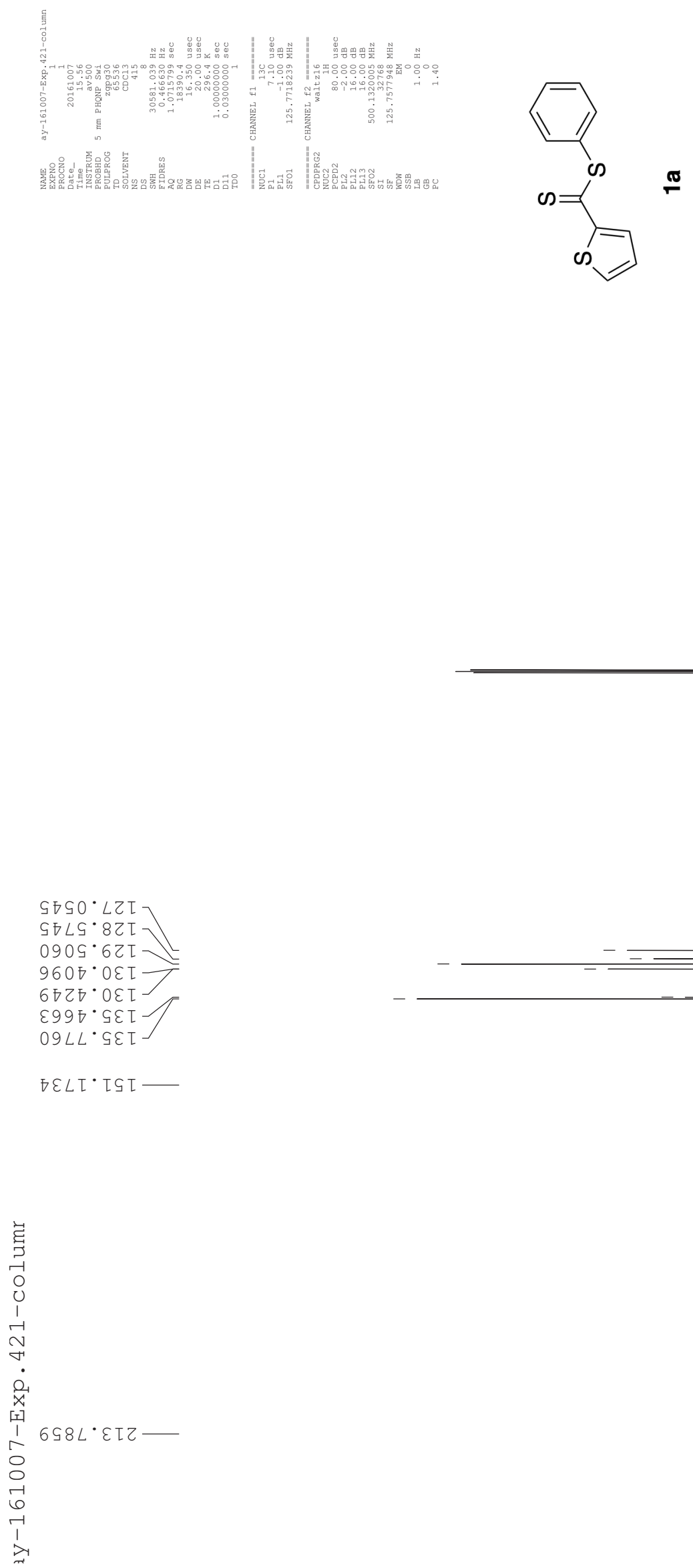
${ }^{1}$ H NMR Spectrum of Phenyl 5-methylthiophene-2-carbodithioate 1b

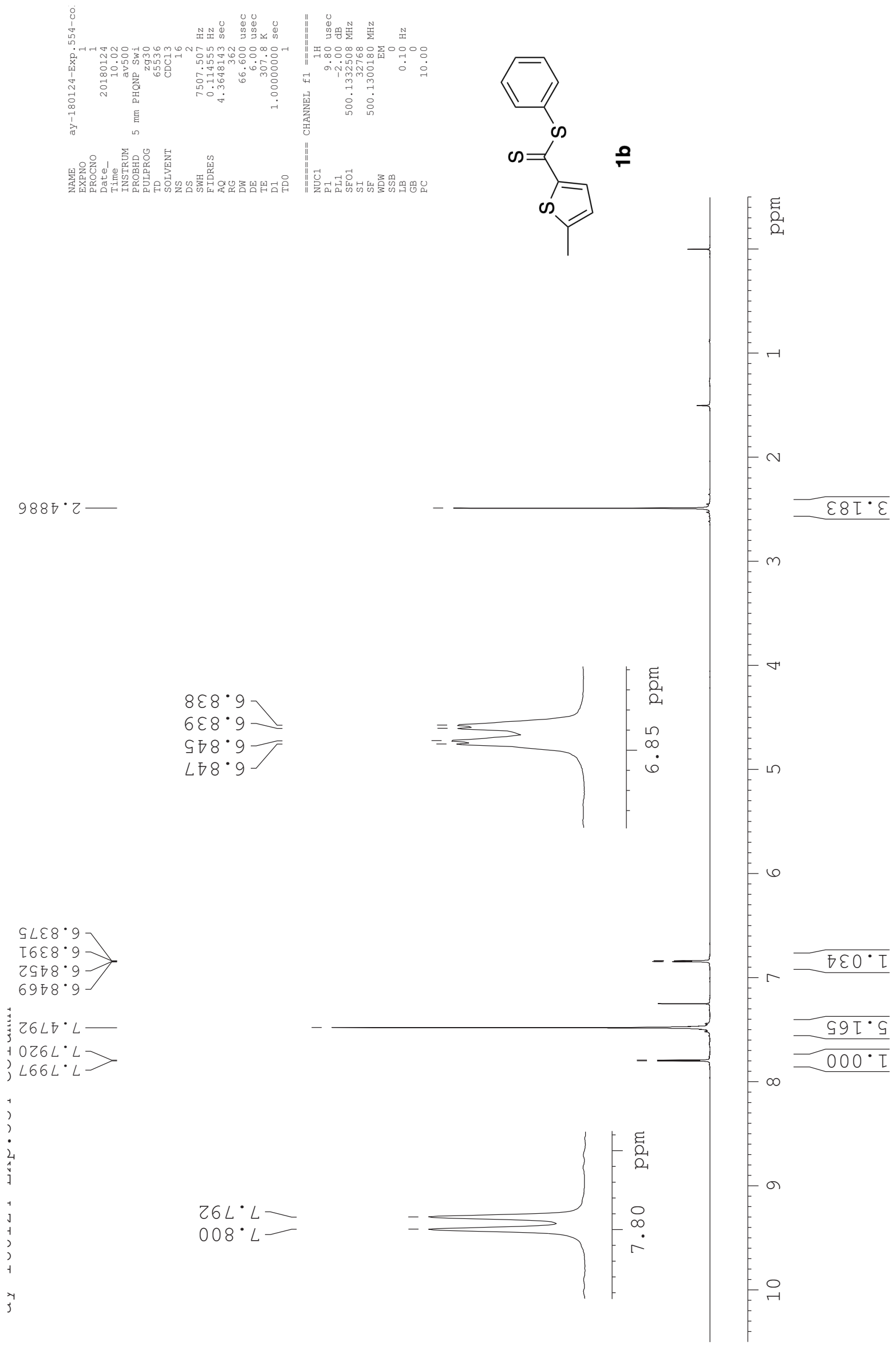


${ }^{13} \mathrm{C}$ NMR Spectrum of Phenyl 5-methylthiophene-2-carbodithioate 1b

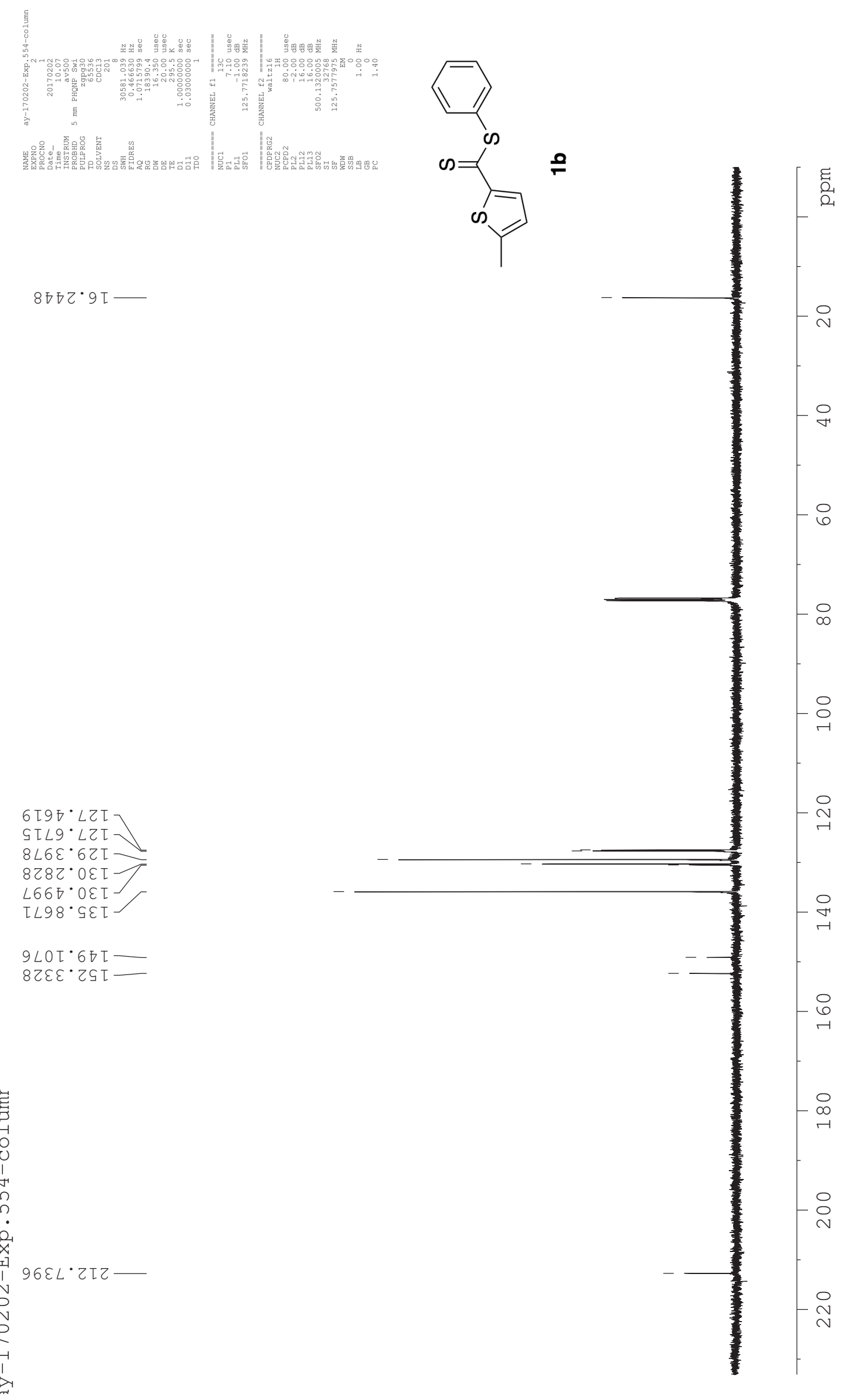


${ }^{1}$ H NMR Spectrum of Phenyl 5-phenylthiophene-2-carbodithioate 1c

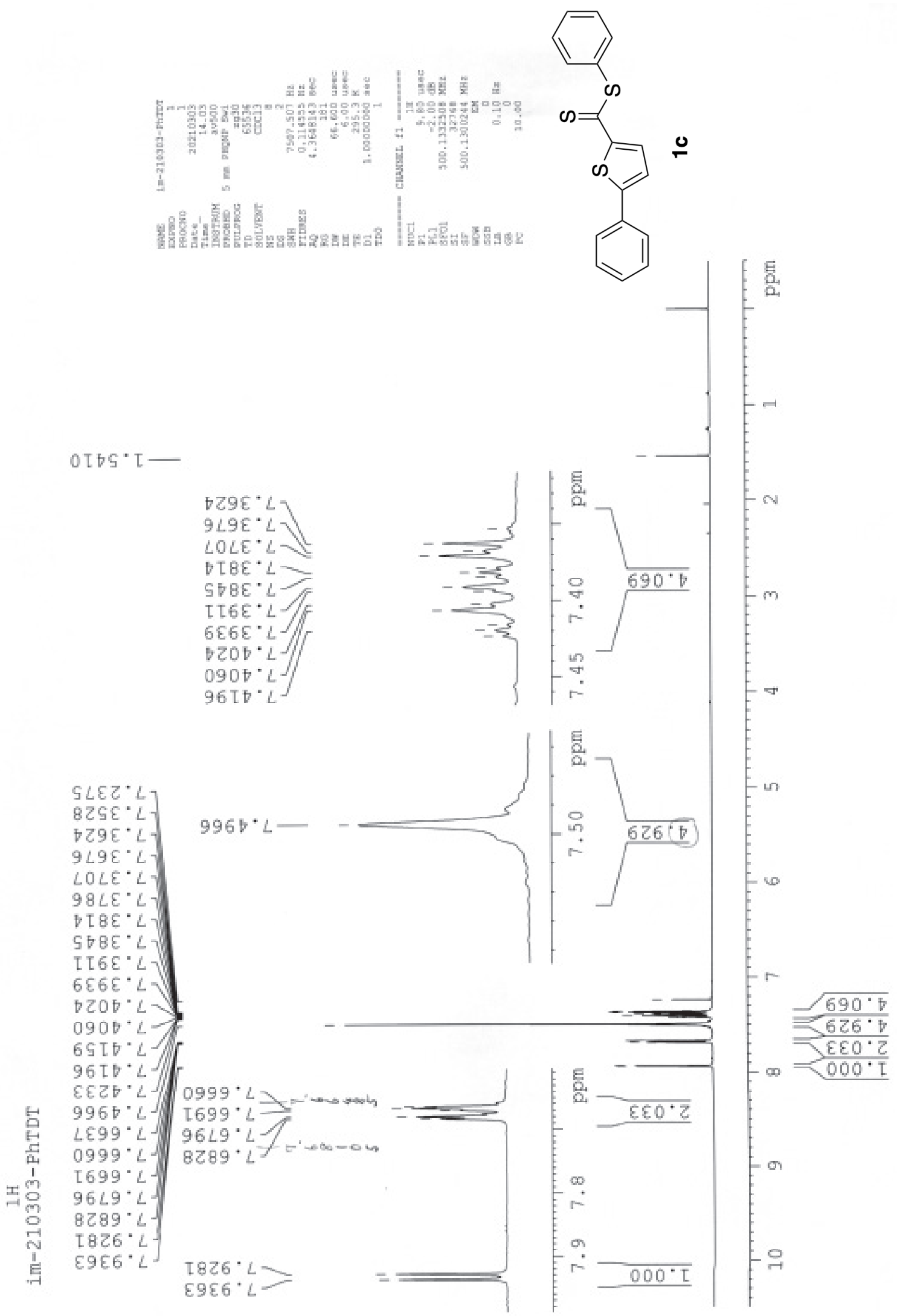


${ }^{13} \mathrm{C}$ NMR Spectrum of Phenyl 5-phenylthiophene-2-carbodithioate 1c
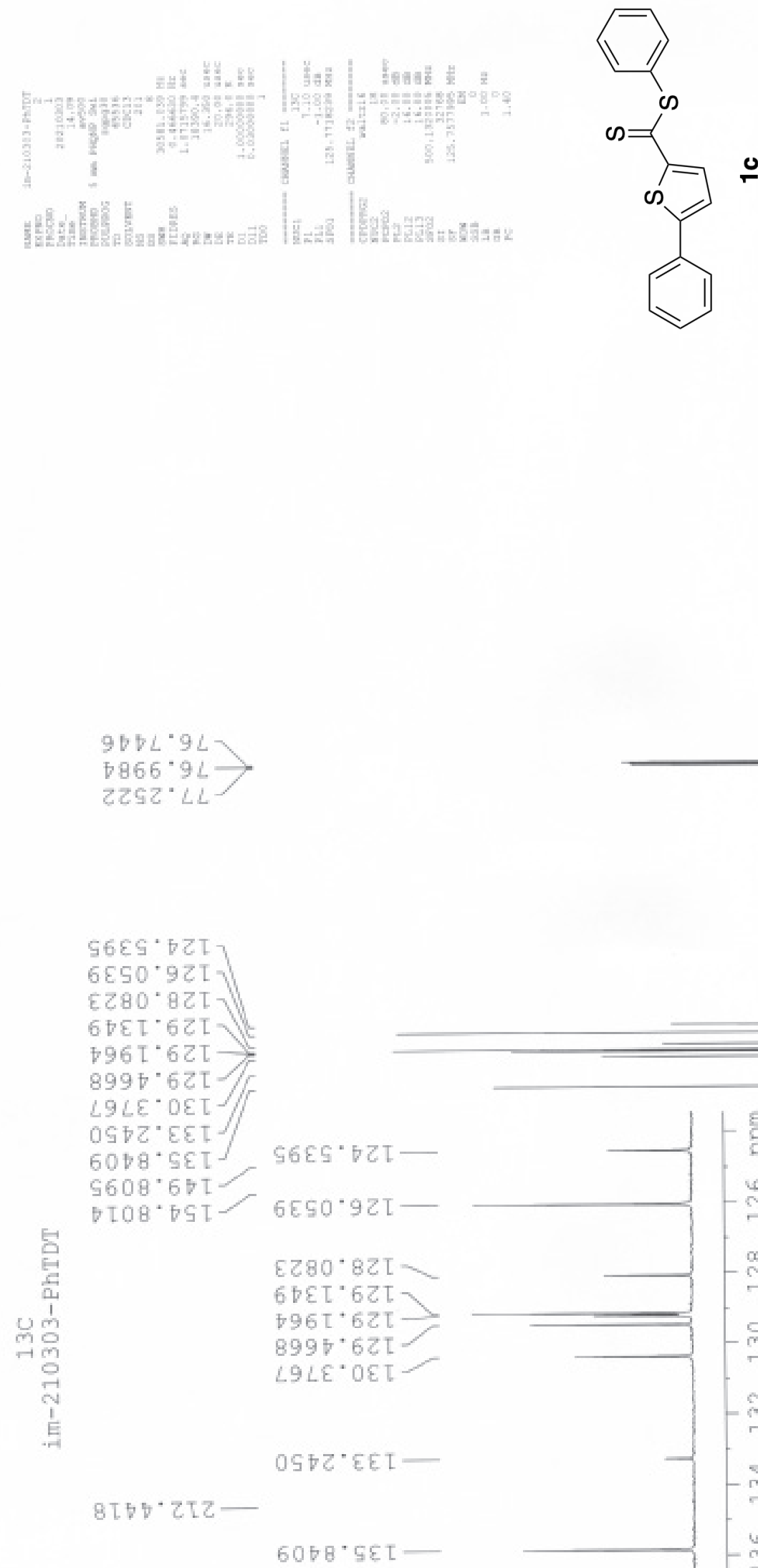

५6हG. б乙T

$6 \varepsilon 50.921$

(2)

L9LE. $0 \varepsilon T$

бobr-set

$6008 \cdot 5 \varepsilon \mathrm{T}$

$5608^{\circ} 6 \mathrm{Bt}$

S6ES ${ }^{\circ}$ DI -

6Еร0.92L-

$2280^{\circ} 82 \mathrm{I}$

$6 \downarrow \varepsilon \tau \cdot 6 Z \tau$

896t 62 t

9LE.0ET

OSฤZ

$6068^{\circ}$ 与ร L - 
${ }^{1}$ H NMR Spectrum of Phenyl benzothiophene-2-carbodithioate 1d

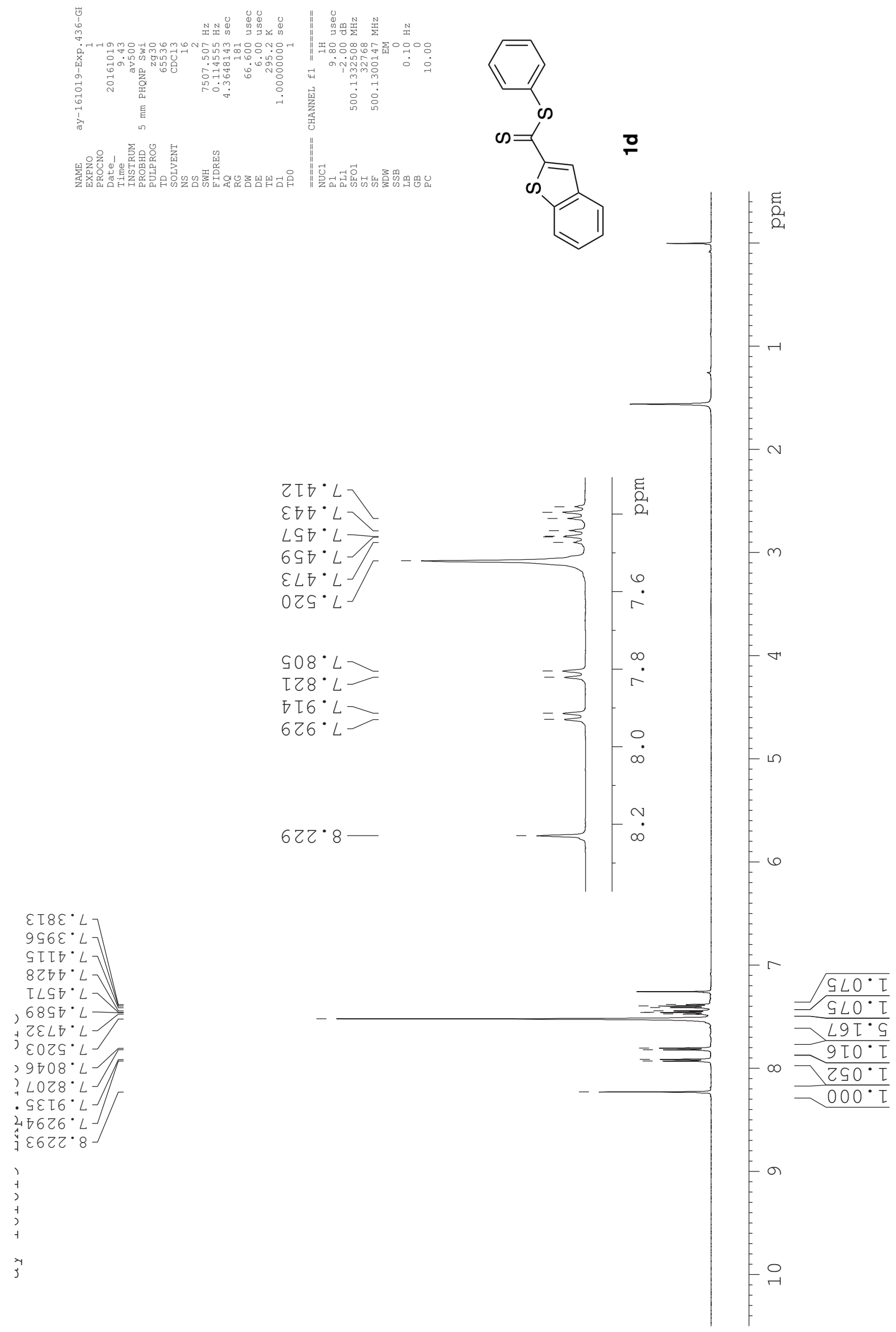


${ }^{13} \mathrm{C}$ NMR Spectrum of Phenyl benzothiophene-2-carbodithioate 1d
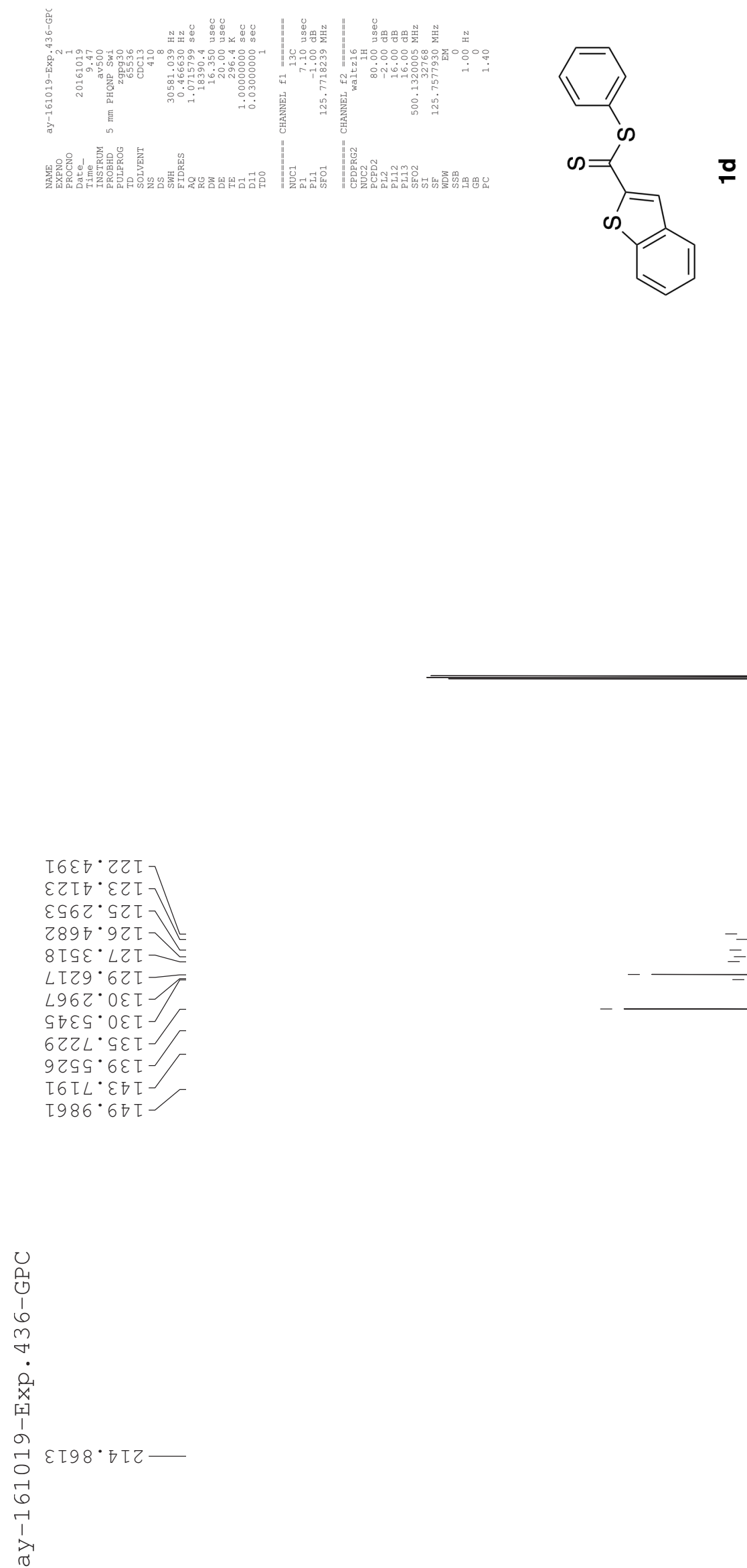
${ }^{1} \mathrm{H}$ NMR Spectrum of Phenyl 5-methylbenzothiophene-2-carbodithioate 1e
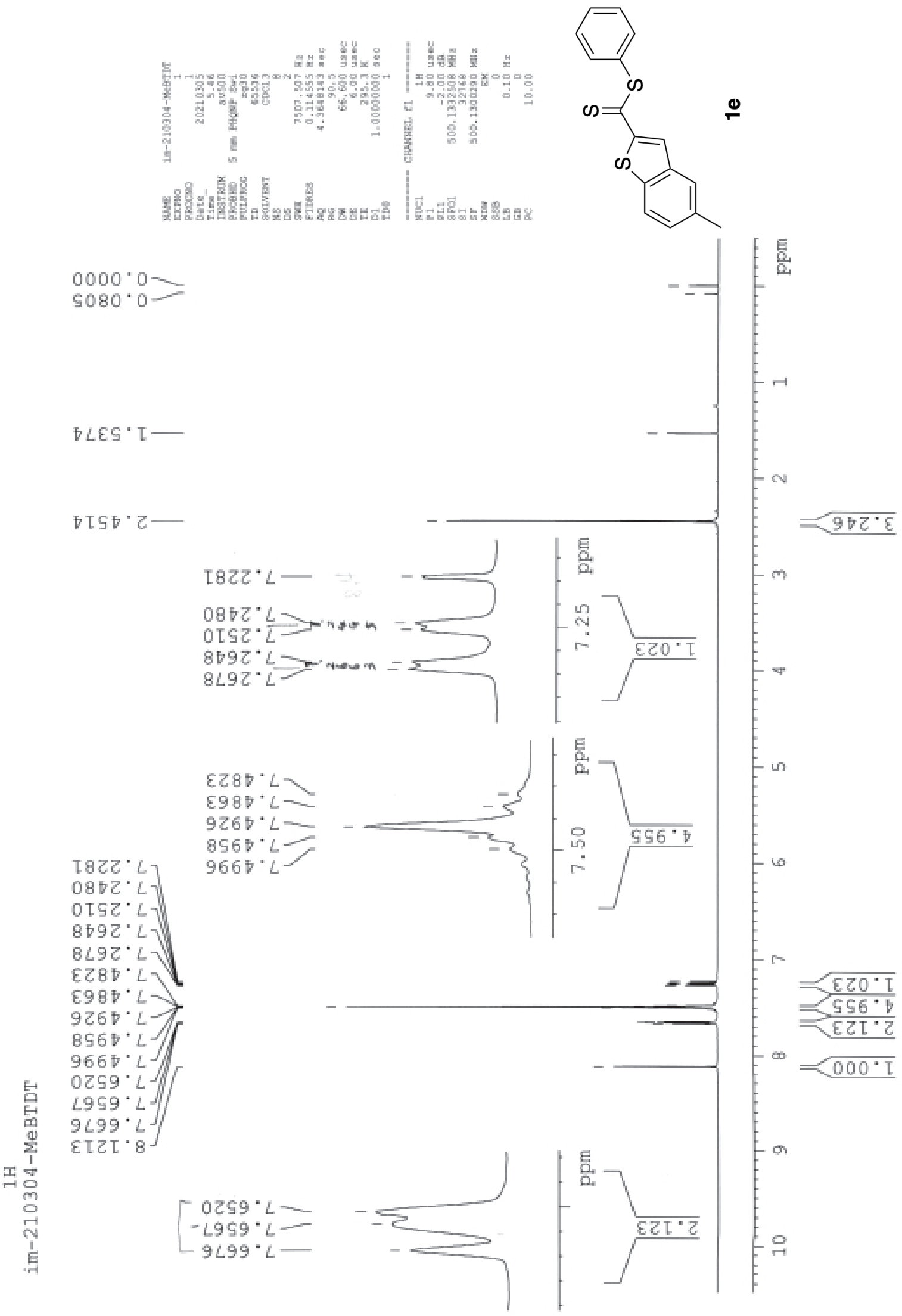
${ }^{13} \mathrm{C}$ NMR Spectrum of Phenyl 5-methylbenzothiophene-2-carbodithioate 1e

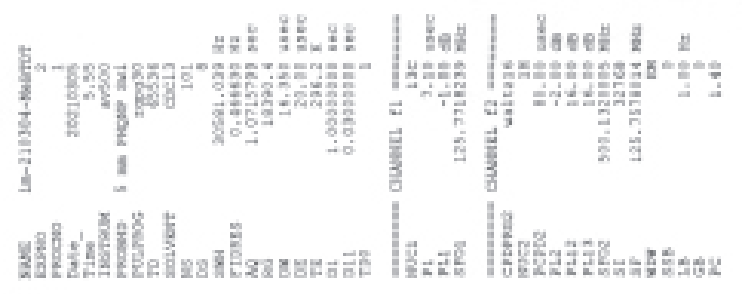

$66 t \varepsilon^{\circ} \tau Z-$

[ $80 L \cdot 9 L$

$6200^{\circ} \angle L$

${ } \angle S Z^{\circ} \angle L{ }^{\prime}$

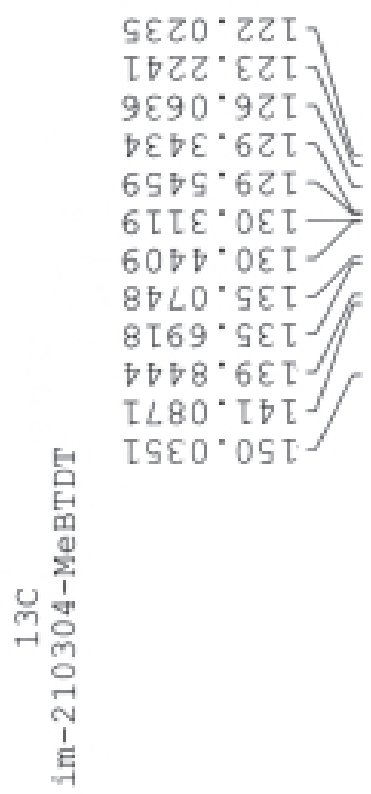

รعzo.zZT—

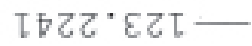

$9 \varepsilon 90 \cdot 9 z \tau-$

$\downarrow \varepsilon \sqcup \varepsilon \cdot 6 Z \tau-$

$6 \mathrm{SbS} \cdot 6 \mathrm{TL}$ -

6 โโ $\varepsilon^{\circ} 0 \varepsilon \tau$

$600 \sigma^{\circ} 0 \varepsilon \tau$

$\angle 89 L^{\circ} \hbar \tau Z-$
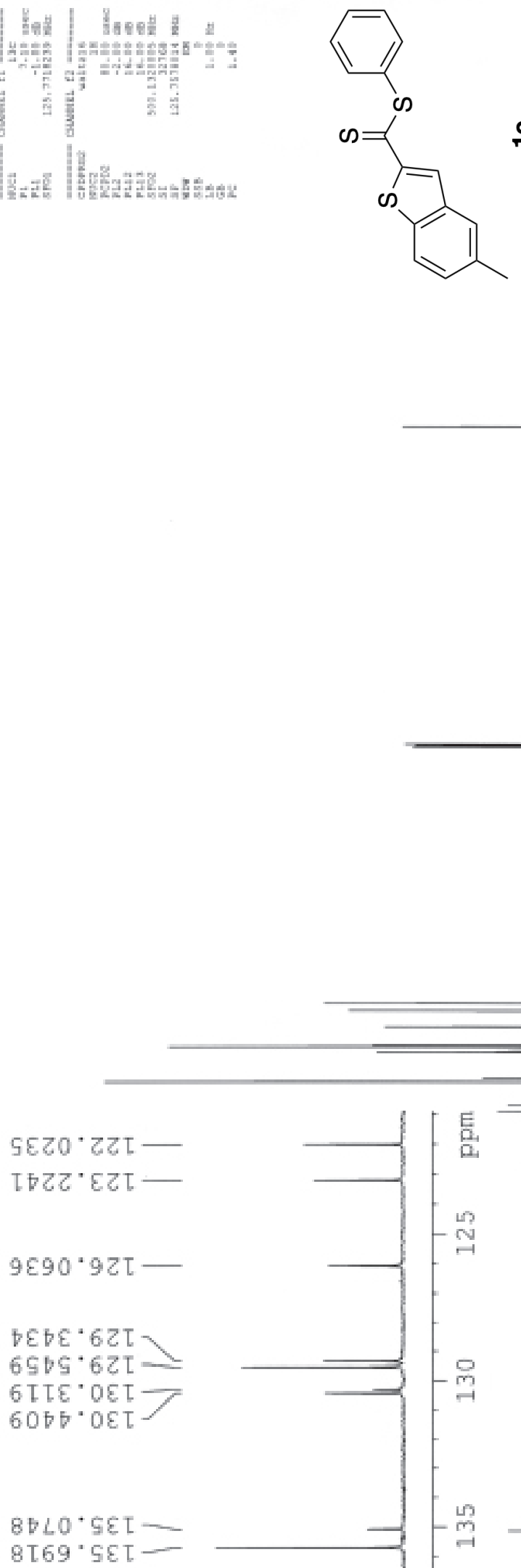

$\stackrel{0}{-}$

果

8

욱 
${ }^{1}$ H NMR Spectrum of Phenyl 5-chlorobenzothiophene-2-carbodithioate $\mathbf{1 f}$
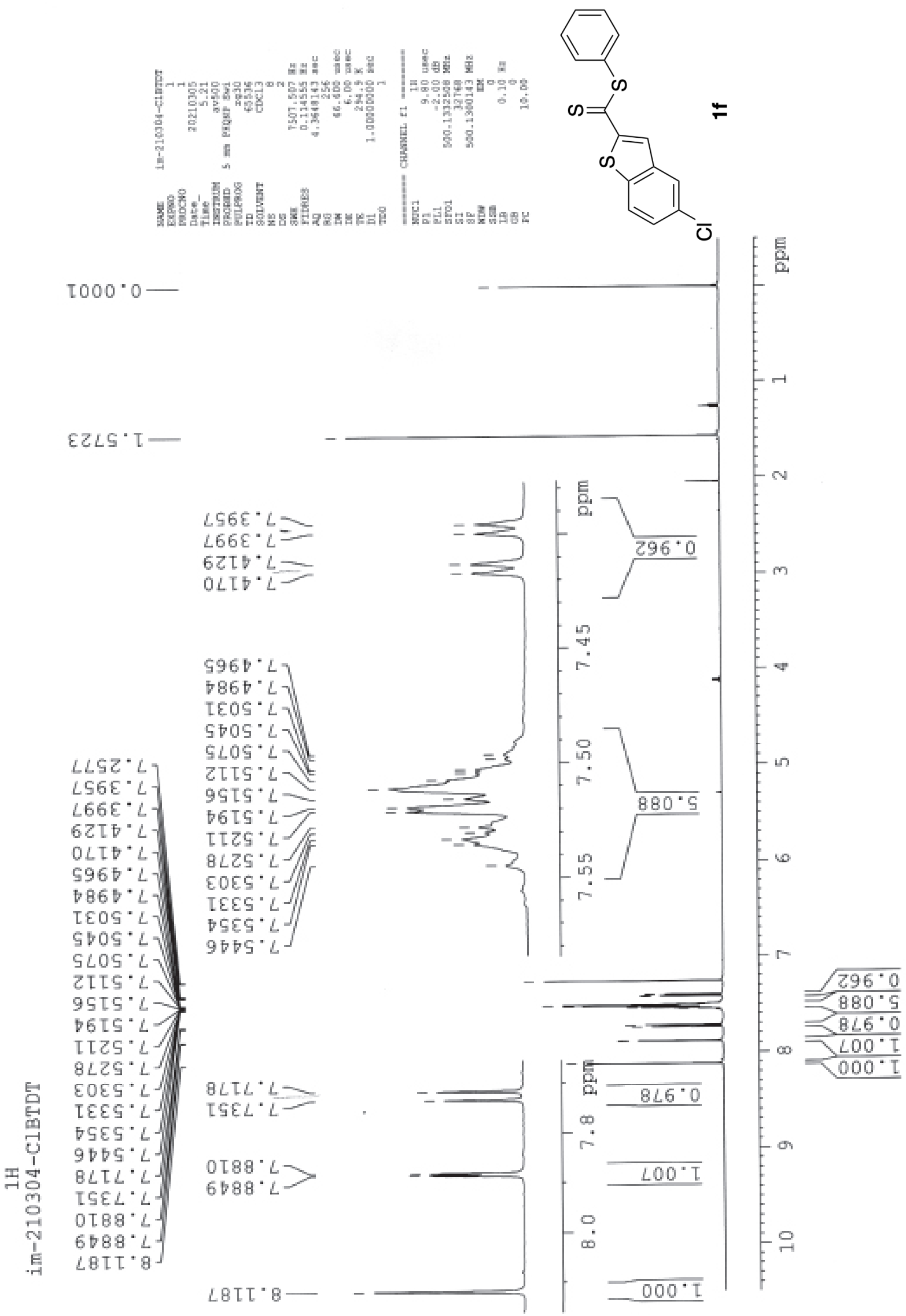
${ }^{13} \mathrm{C}$ NMR Spectrum of Phenyl 5-chlorobenzothiophene-2-carbodithioate $\mathbf{1 f}$
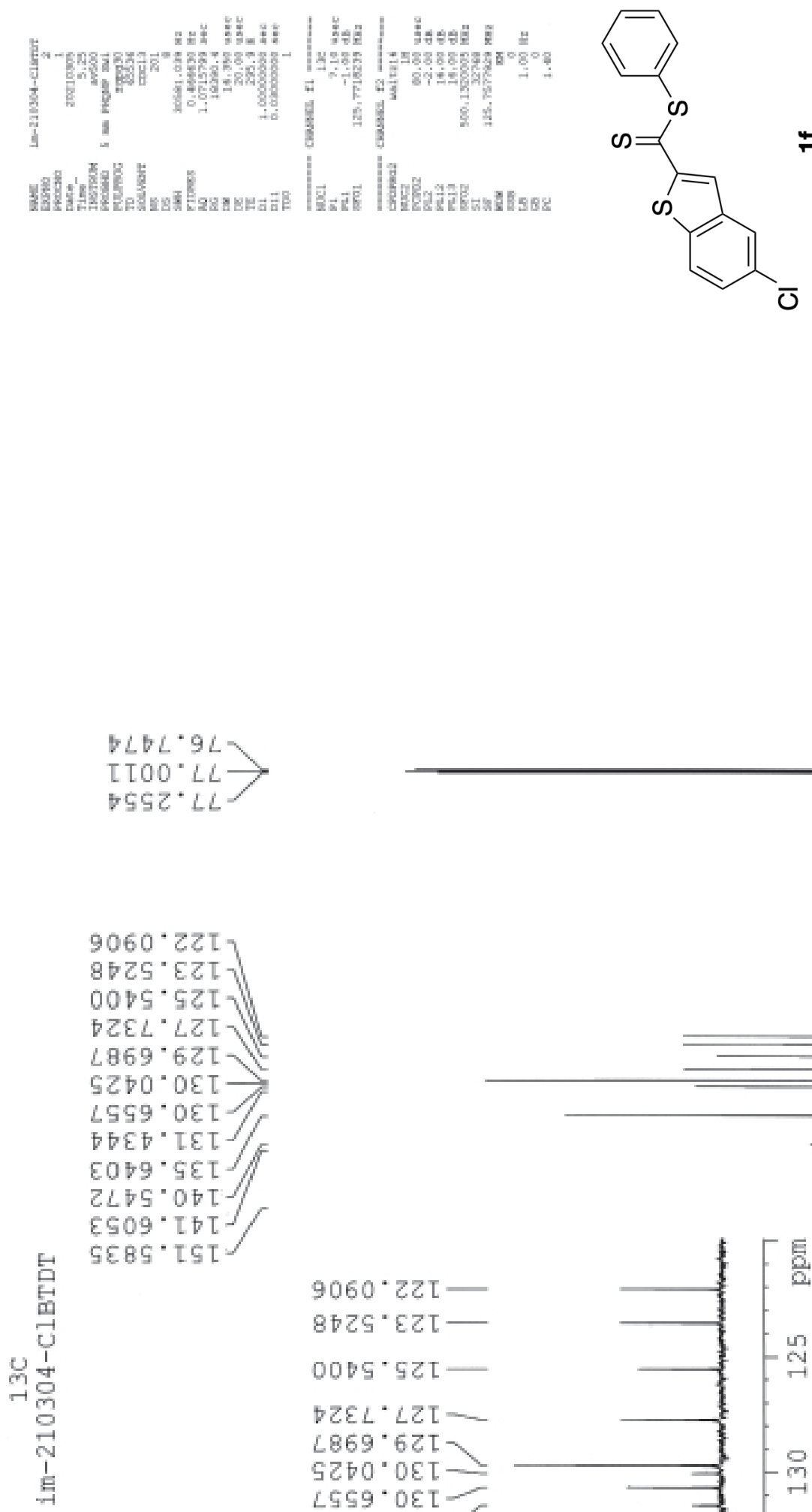

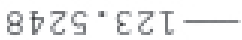

$000 \mathrm{~S}^{\circ} \mathrm{GZT}$

$\nabla Z \varepsilon L^{\circ} \angle Z \mathrm{~L}$ $\angle 869^{\circ} 6 \mathrm{ZT}$ SZ70 $0 E T$ LSS9 ${ }^{\circ}$ OET $\nabla \downarrow \varepsilon \nabla^{\circ} \tau \varepsilon \tau$

$0 \angle S 9^{\circ} \nabla \tau 2-$ $\varepsilon 009^{*} \varsigma \varepsilon \tau-$ 
${ }^{1} \mathrm{H}$ NMR Spectrum of 2-Bromophenyl benzothiophene-2-carbodithioate $\mathbf{1 g}$

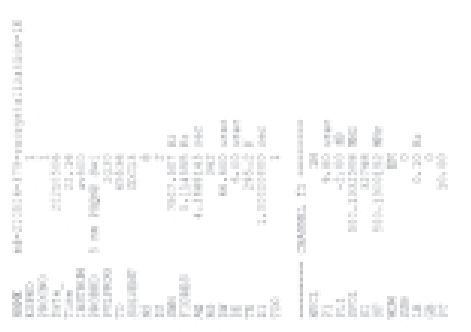
$0000^{\circ} 0$
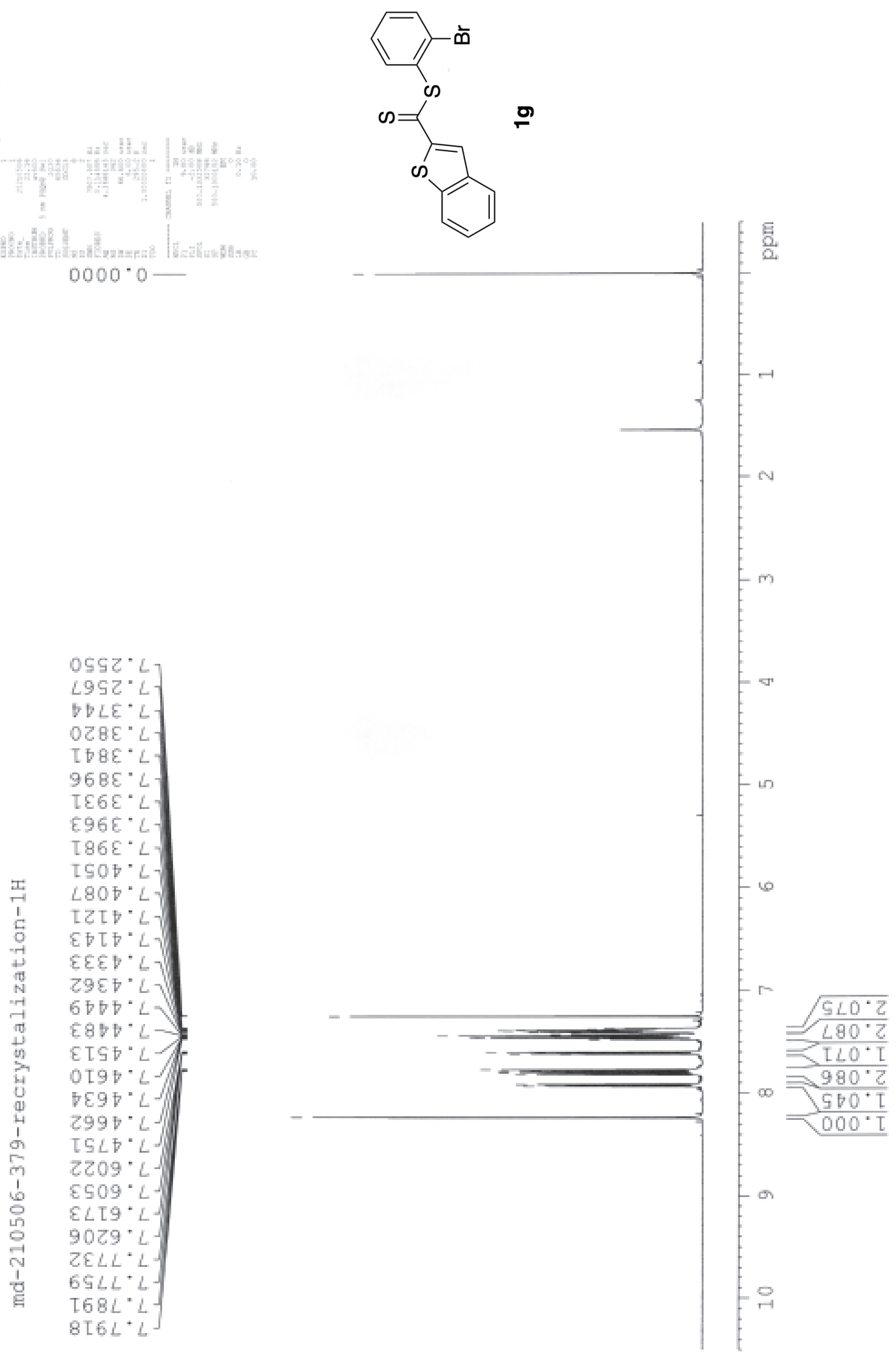
${ }^{13} \mathrm{C}$ NMR Spectrum of 2-Bromophenyl benzothiophene-2-carbodithioate $\mathbf{1 g}$

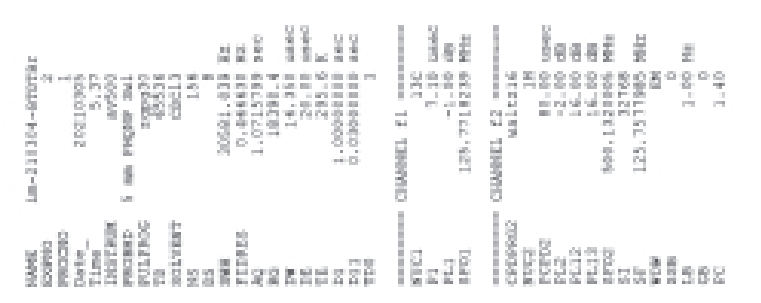<smiles>O=C(Oc1ccccc1Br)c1cc2ccccc2o1</smiles>

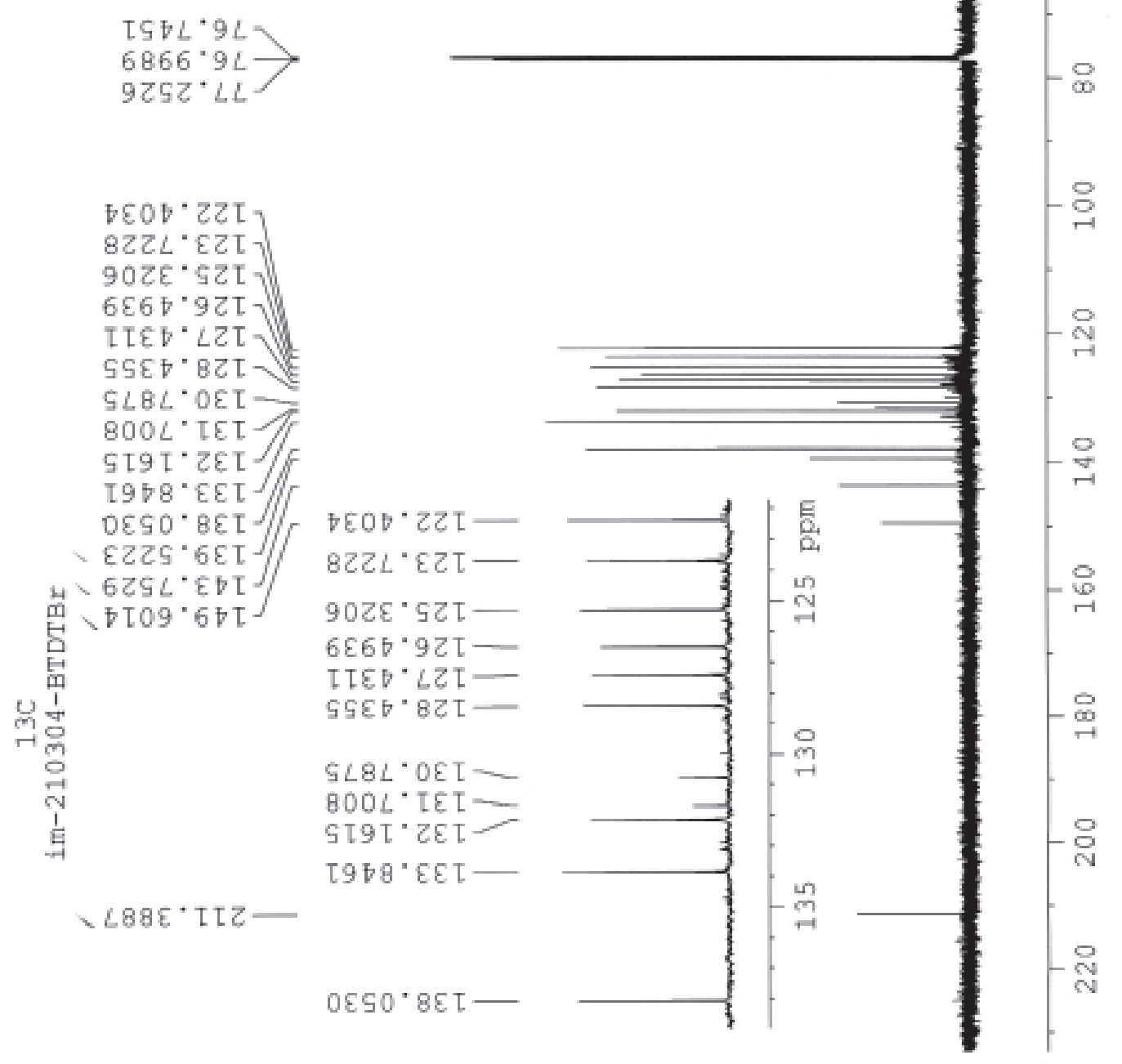


${ }^{1} \mathrm{H}$ NMR Spectrum of Phenyl thiophene-3-carbodithioate $\mathbf{1 h}$
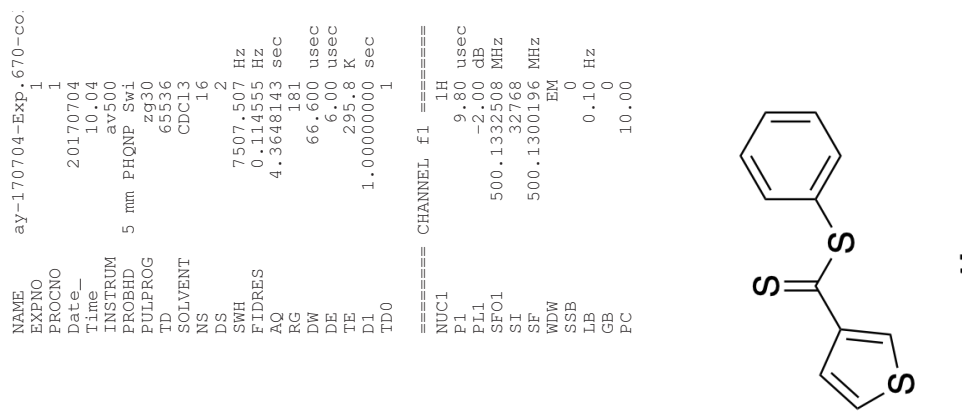

$\mp$
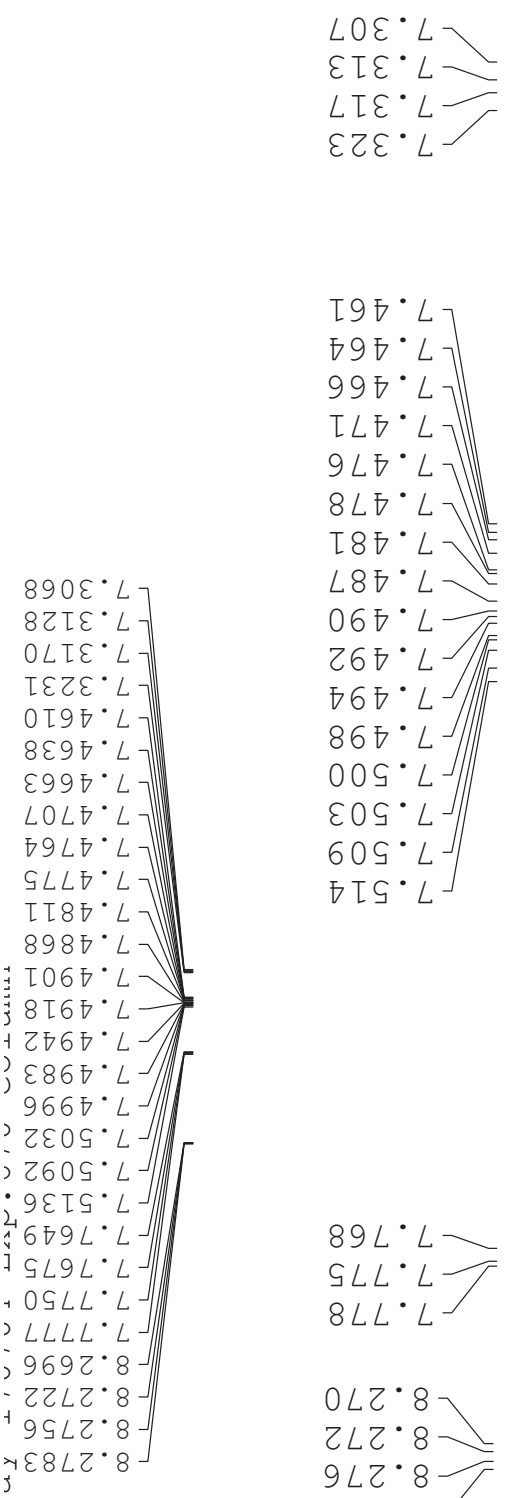

$$
\text { ธโS. L }
$$

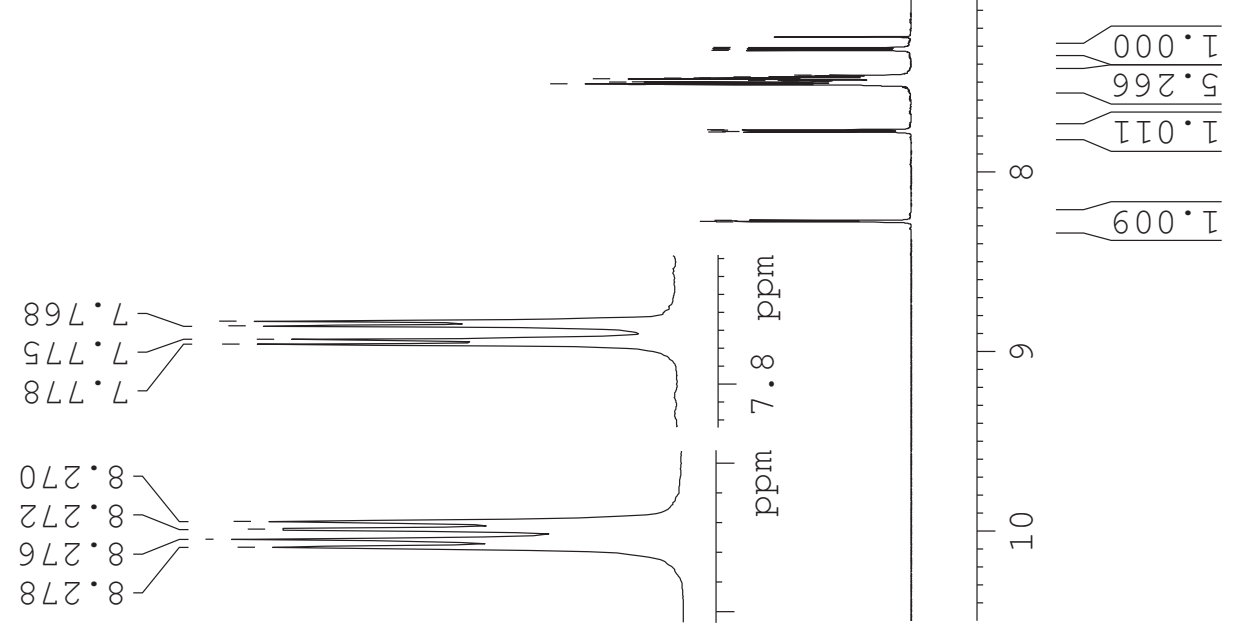


${ }^{13} \mathrm{C}$ NMR Spectrum of Phenyl thiophene-3-carbodithioate $\mathbf{1 h}$
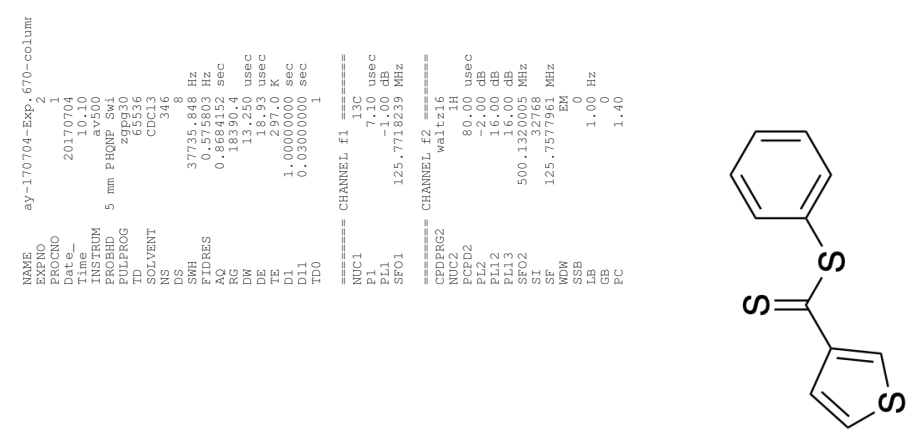

F
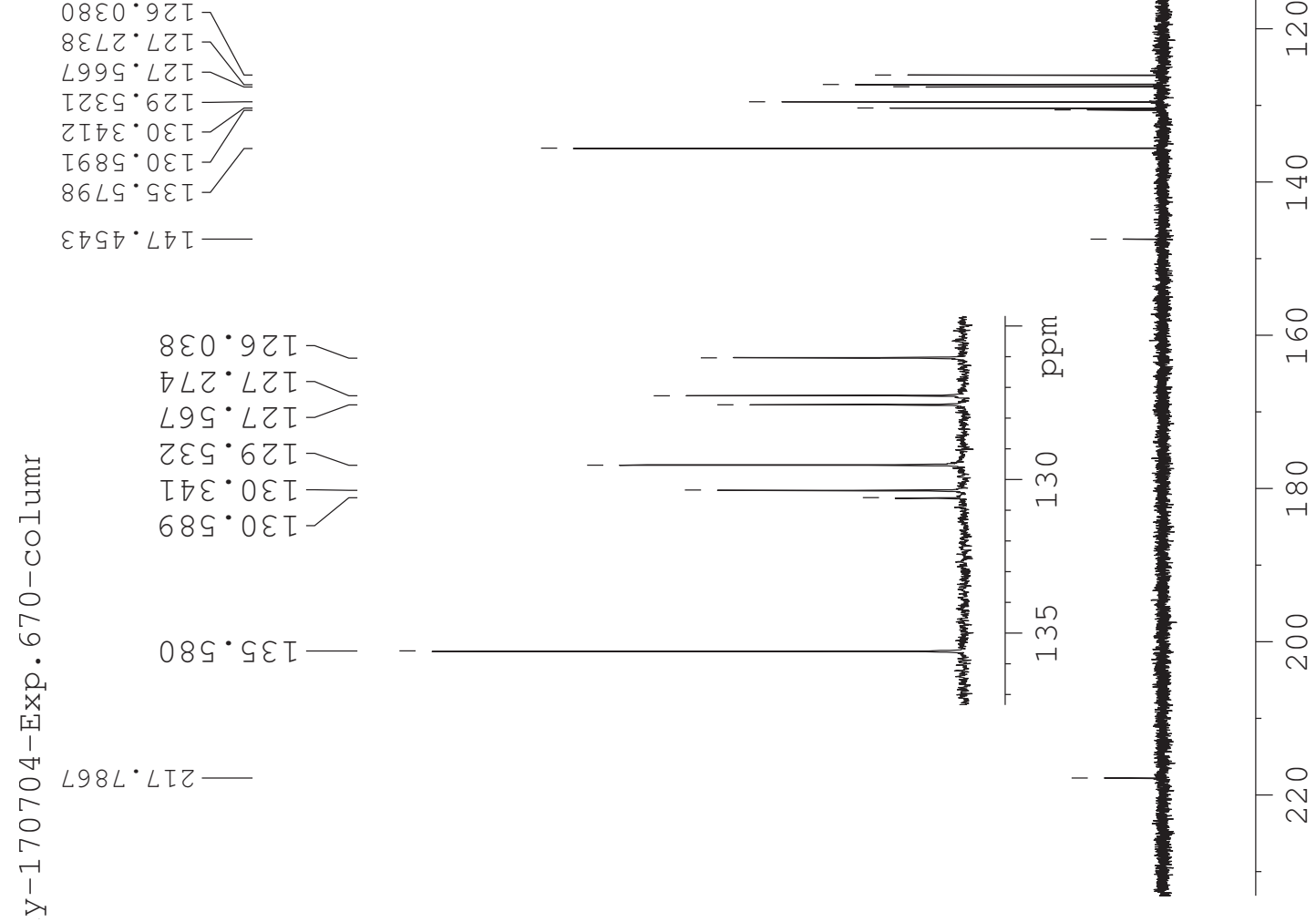
${ }^{1} \mathrm{H}$ NMR Spectrum of Phenyl 5-methylthiophene-3-carbodithioate 1i

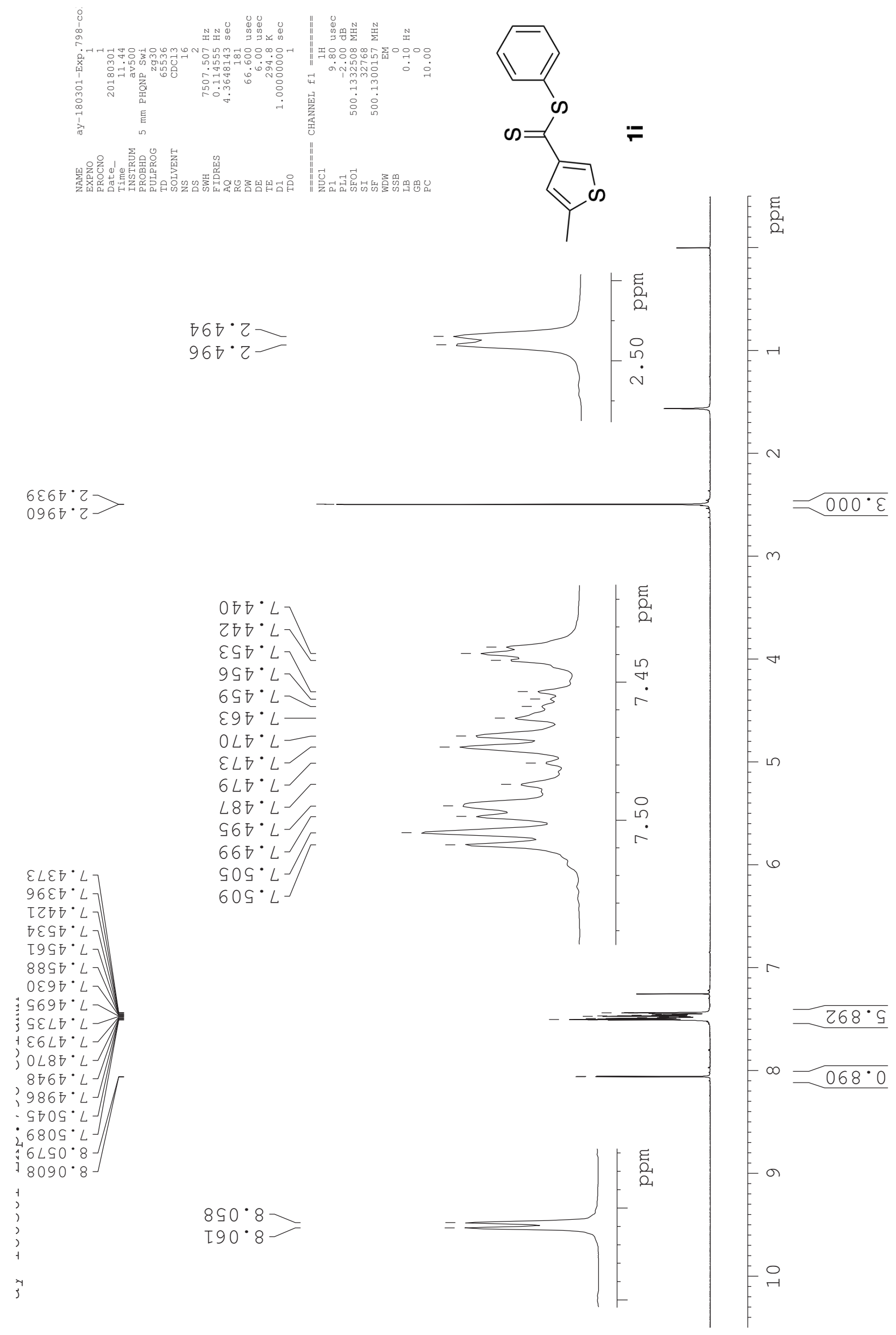


${ }^{13} \mathrm{C}$ NMR Spectrum of Phenyl 5-methylthiophene-3-carbodithioate 1i
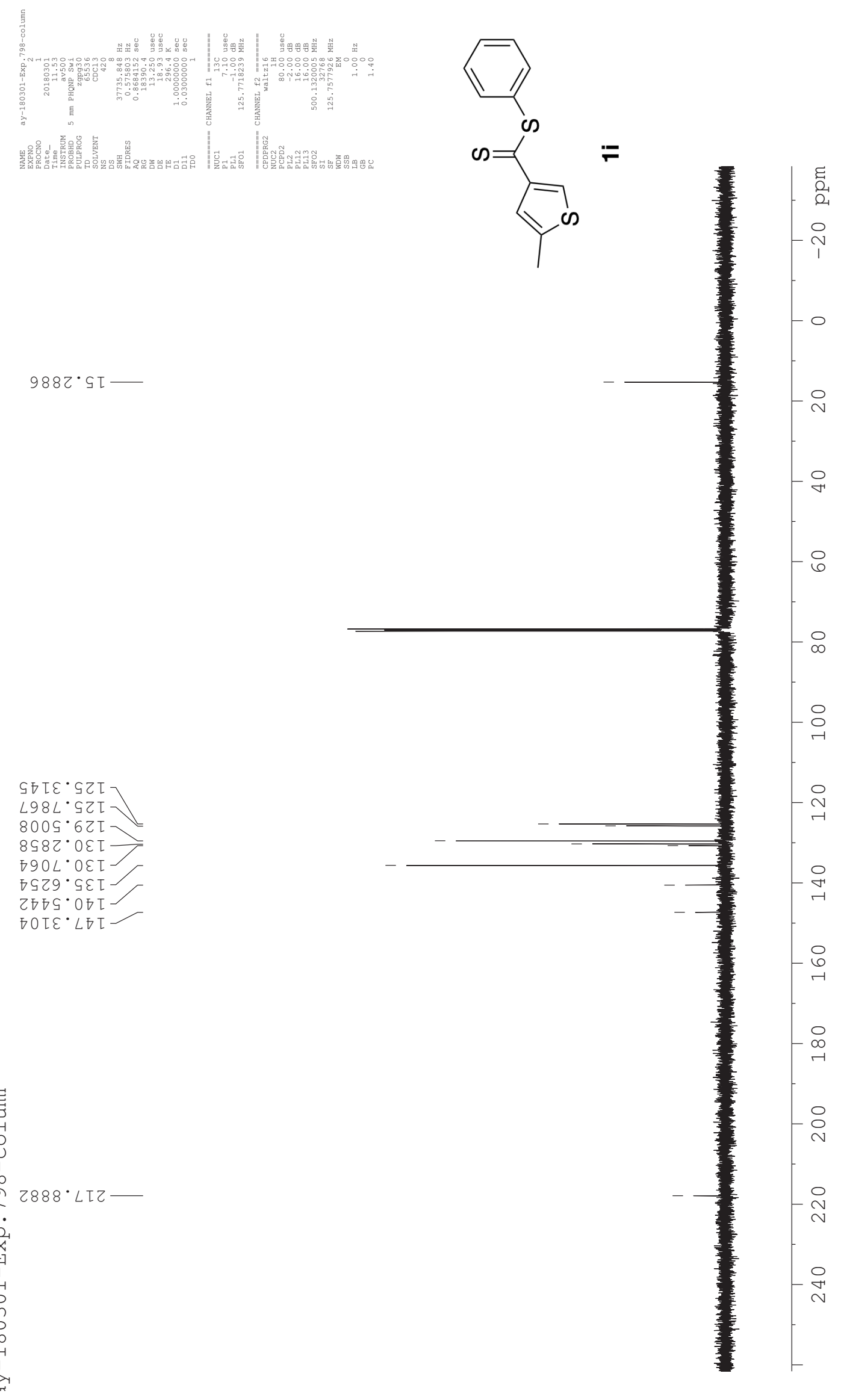
${ }^{1} \mathrm{H}$ NMR Spectrum of Phenyl 5-phenylthiophene-3-carbodithioate $\mathbf{1 j}$

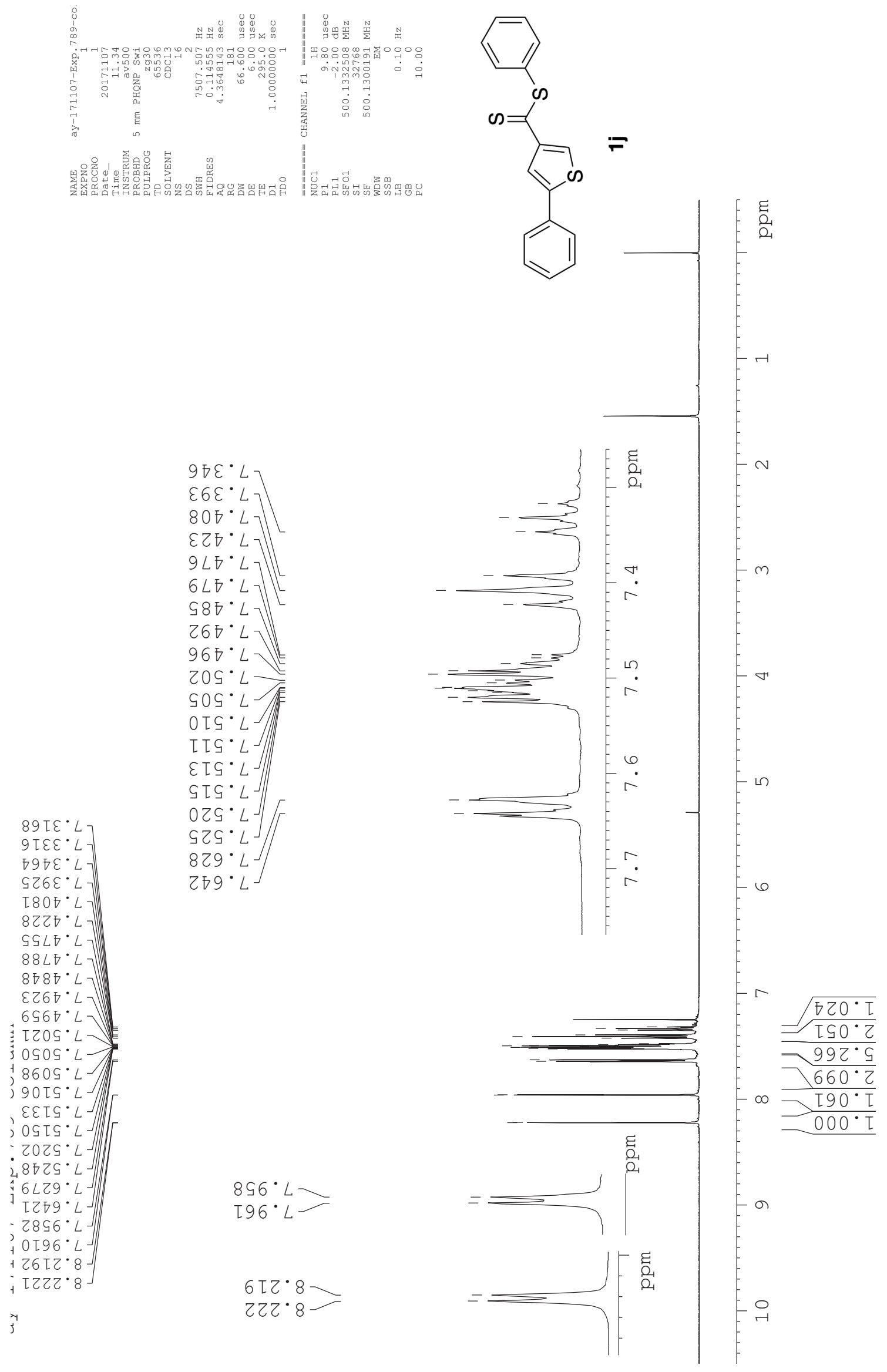


${ }^{13} \mathrm{C}$ NMR Spectrum of Phenyl 5-phenylthiophene-3-carbodithioate $\mathbf{1 j}$
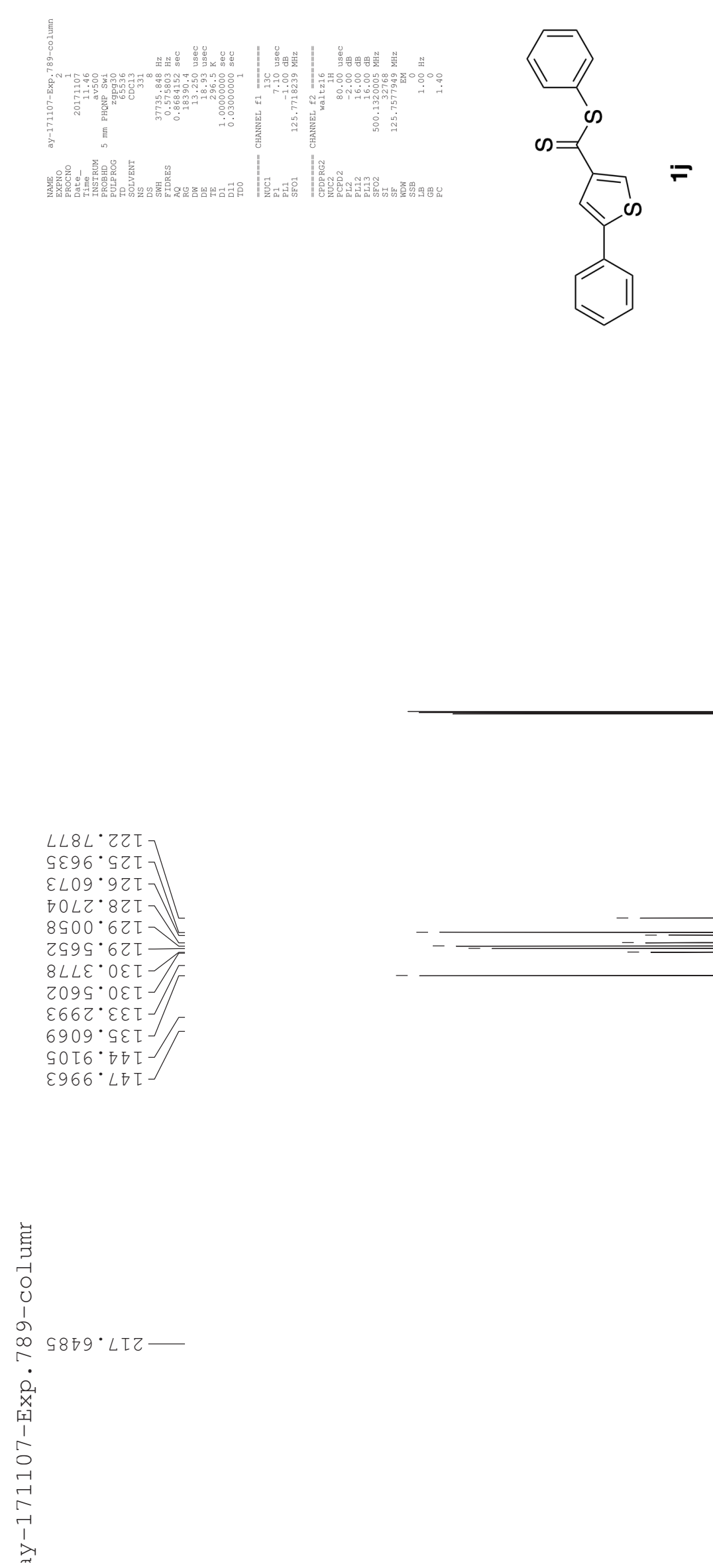
${ }^{1}$ H NMR Spectrum of Phenyl 5-bromothiophene-3-carbodithioate 1k

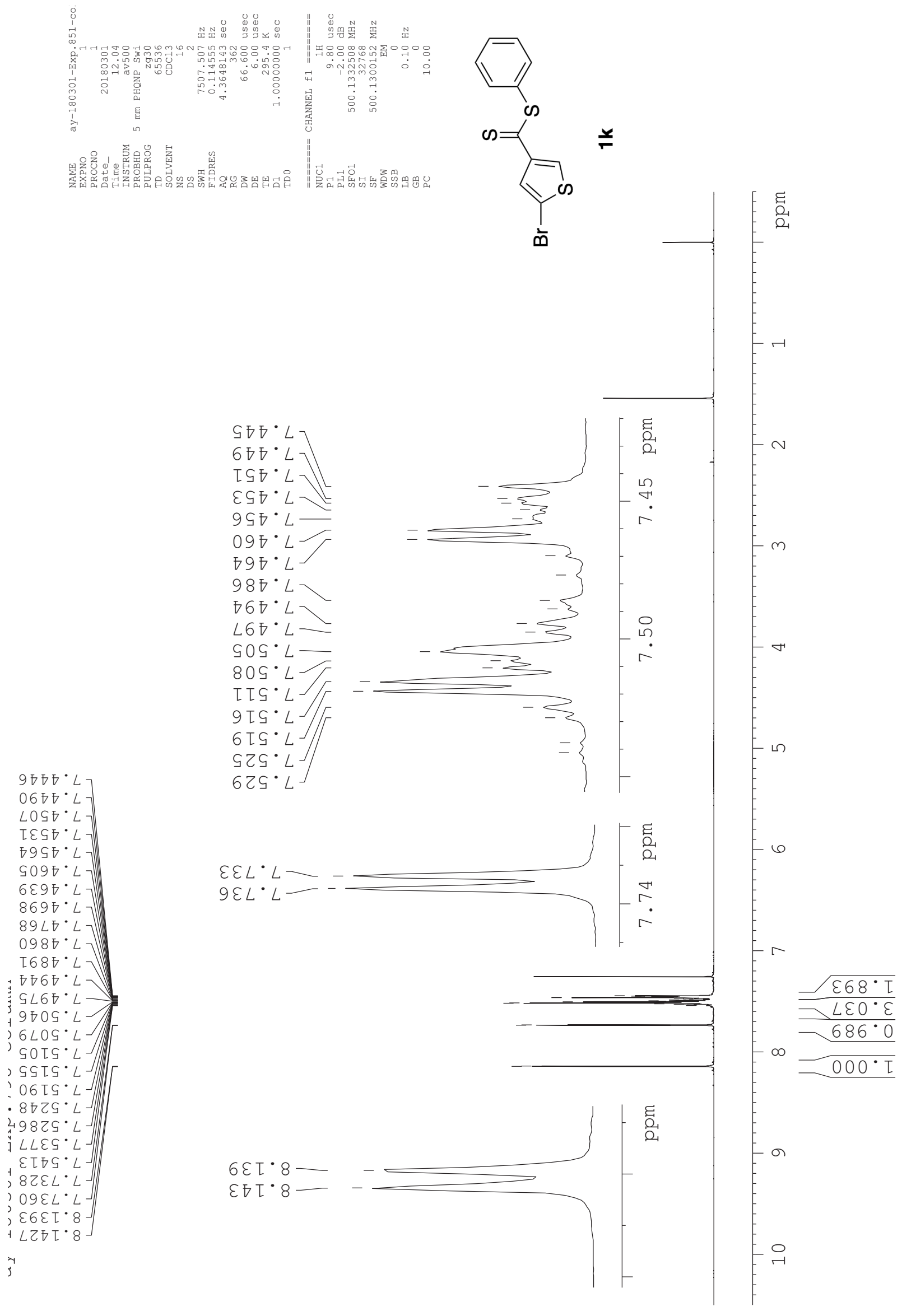


${ }^{13} \mathrm{C}$ NMR Spectrum of Phenyl 5-bromothiophene-3-carbodithioate $\mathbf{1 k}$

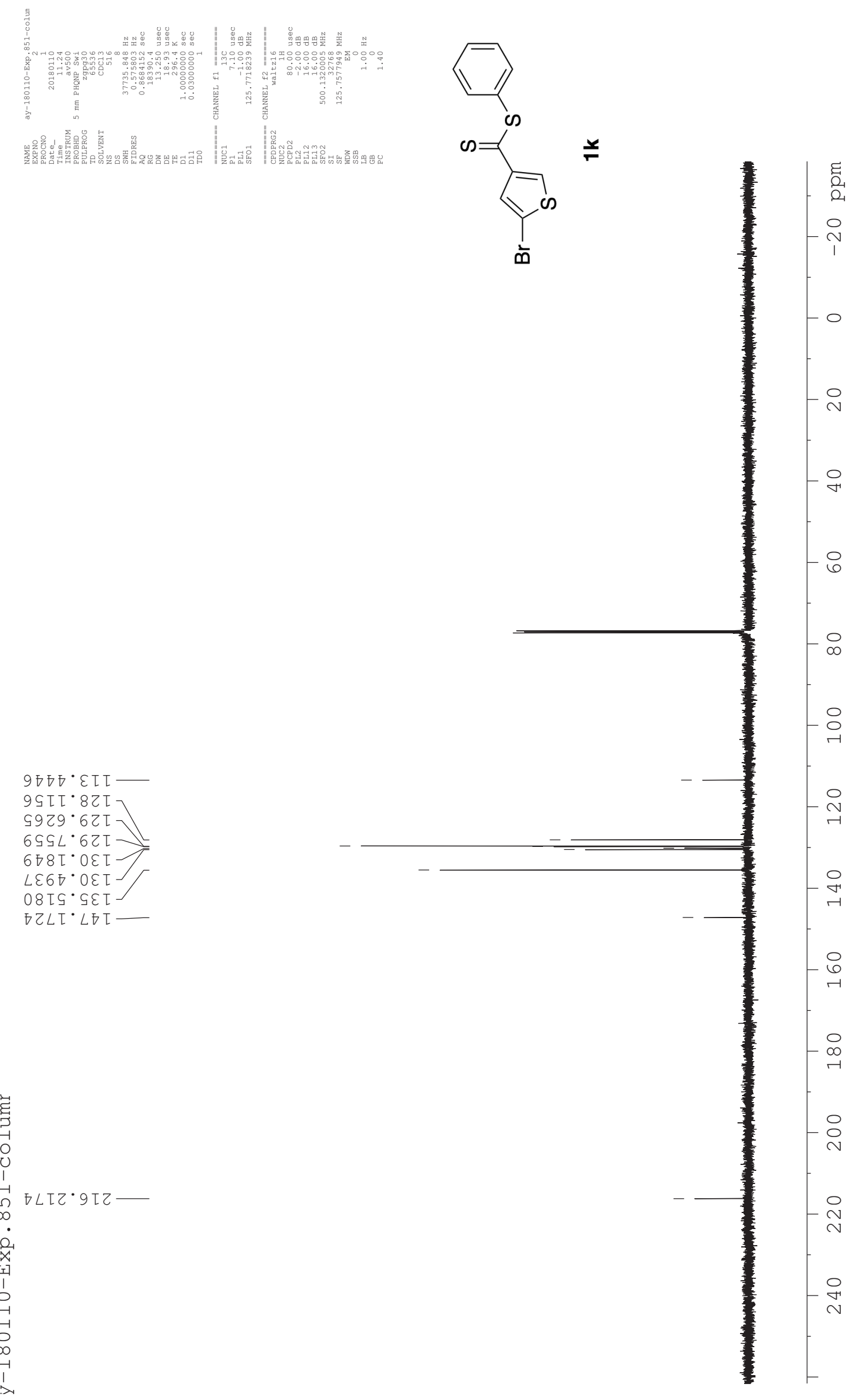


${ }^{1}$ H NMR Spectrum of 4-Methoxyphenyl thiophene-3-carbodithioate $\mathbf{1 l}$

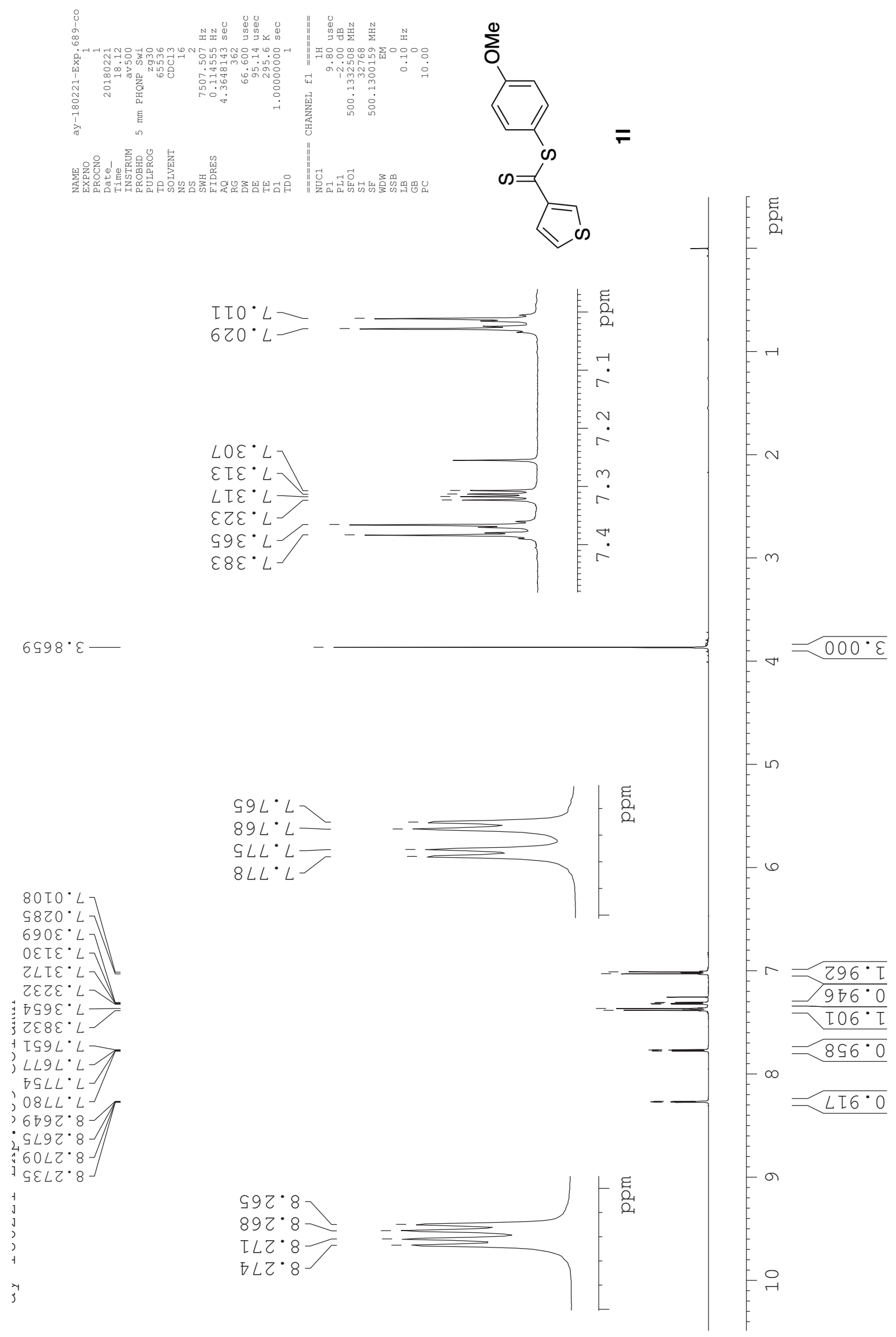


${ }^{13}$ C NMR Spectrum of 4-Methoxyphenyl thiophene-3-carbodithioate $\mathbf{1 I}$

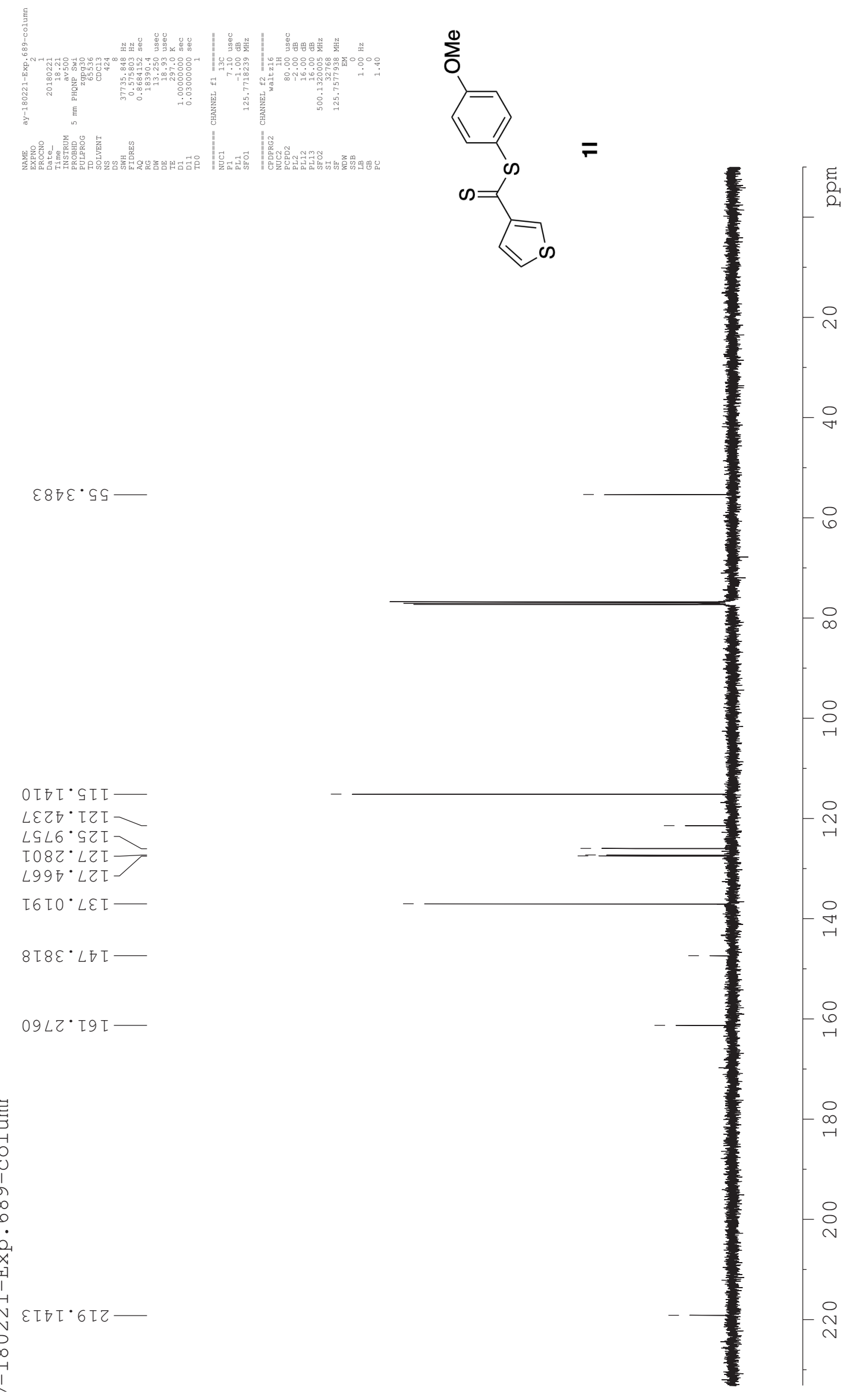


${ }^{1}$ H NMR Spectrum of 4-Chlorophenyl thiophene-3-carbodithioate 1m
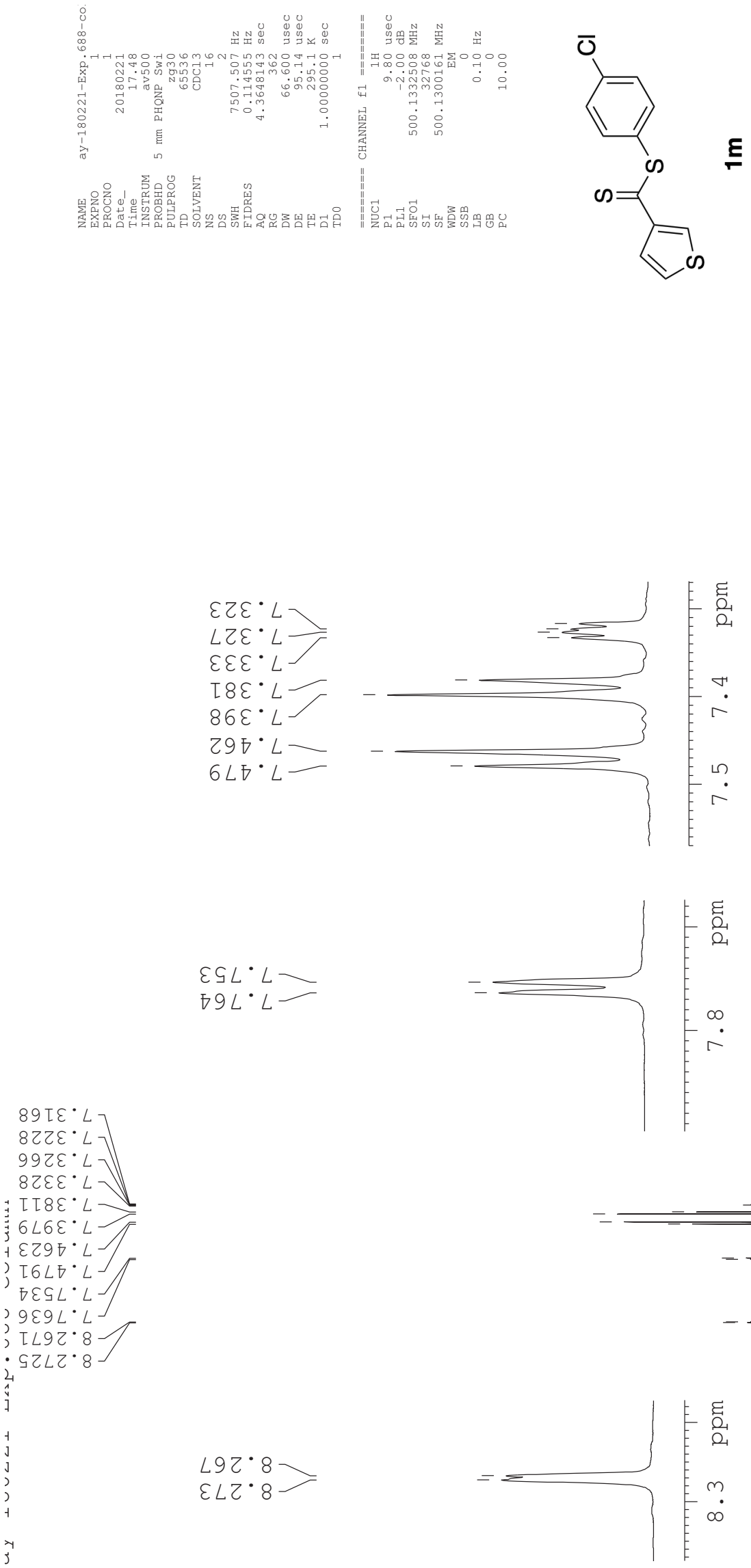

$\angle 92 \cdot 8$

$\varepsilon\llcorner 乙 \cdot 8>$
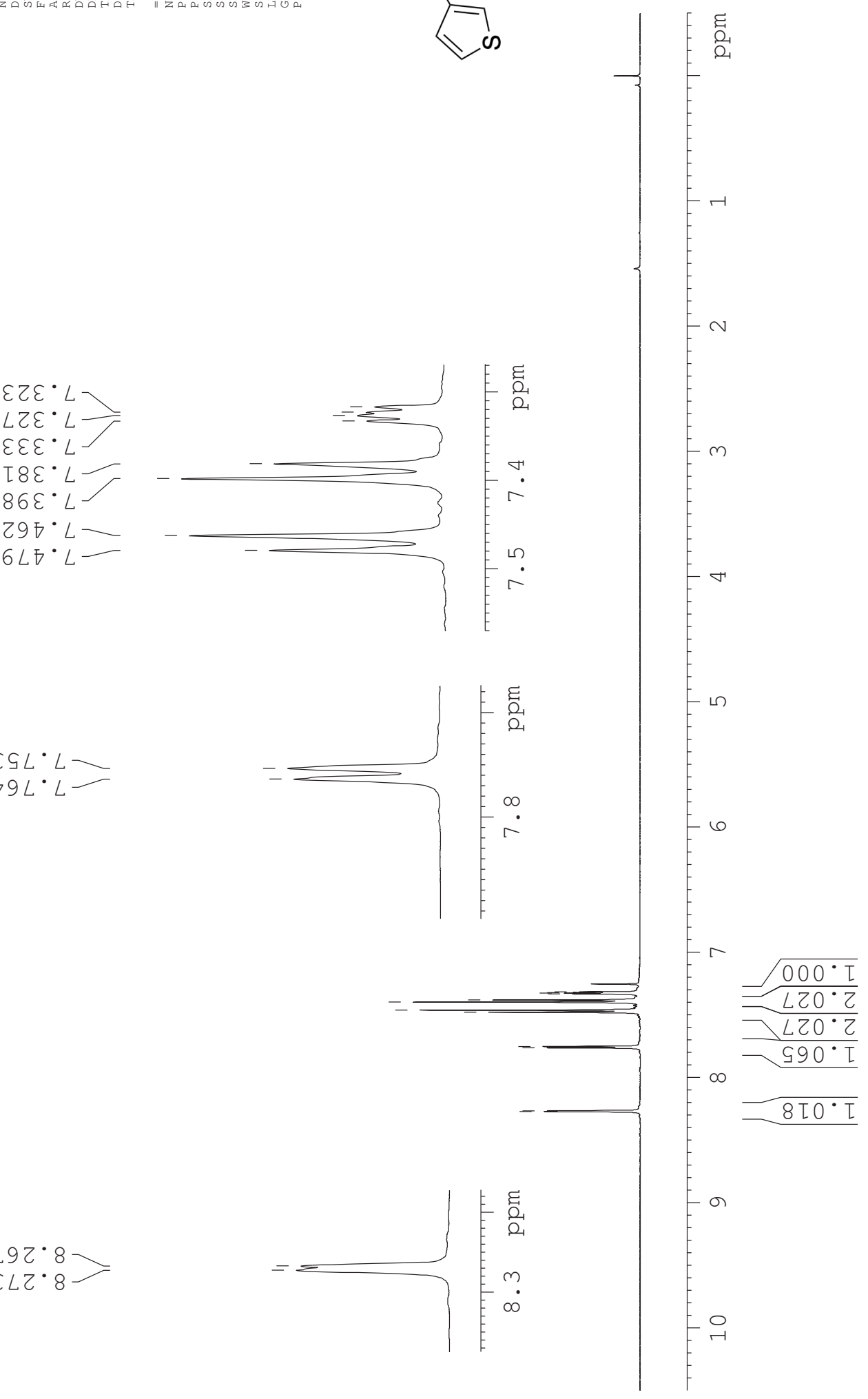
$\varepsilon \varepsilon \varepsilon^{\bullet}\llcorner$

โ8 $\varepsilon^{\circ}$

$86 \varepsilon^{\cdot} L$

$29 \nabla^{\cdot}$

$6 \angle \nabla \cdot L$ 9 $L^{\circ} L$ 
${ }^{13} \mathrm{C}$ NMR Spectrum of 4-Chlorophenyl thiophene-3-carbodithioate $\mathbf{1 m}$
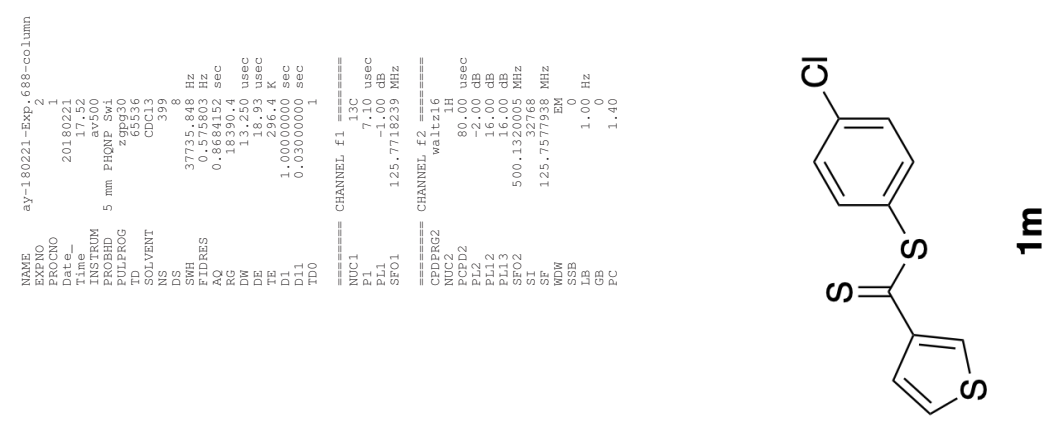

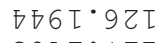

S0万乙・ LZT

$996 L \cdot L Z T$

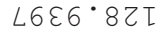

โ098.6ZT

$0<88 \cdot 9 \varepsilon \tau$

$\nabla T O \varepsilon \cdot \operatorname{LT}$
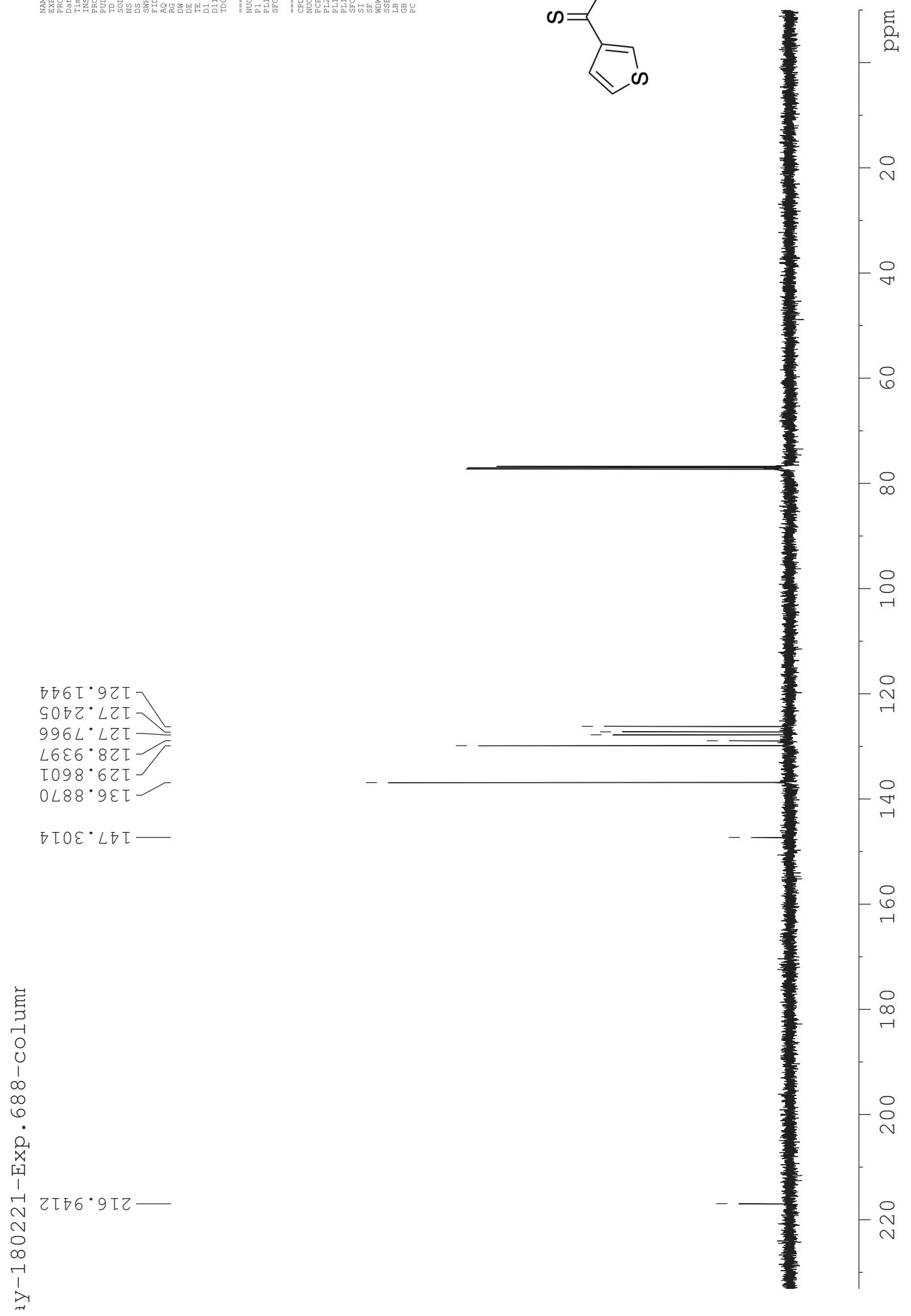
${ }^{1} \mathrm{H}$ NMR Spectrum of Phenyl benzothiophene-3-carbodithioate 1n
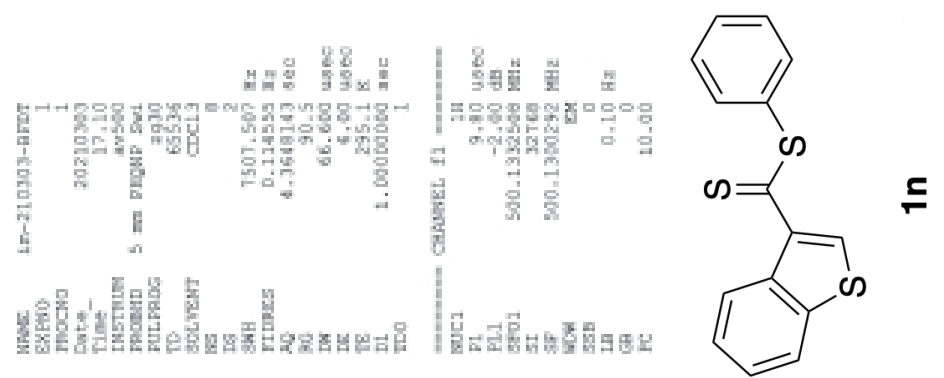

$\tau 000^{\circ} 0-$

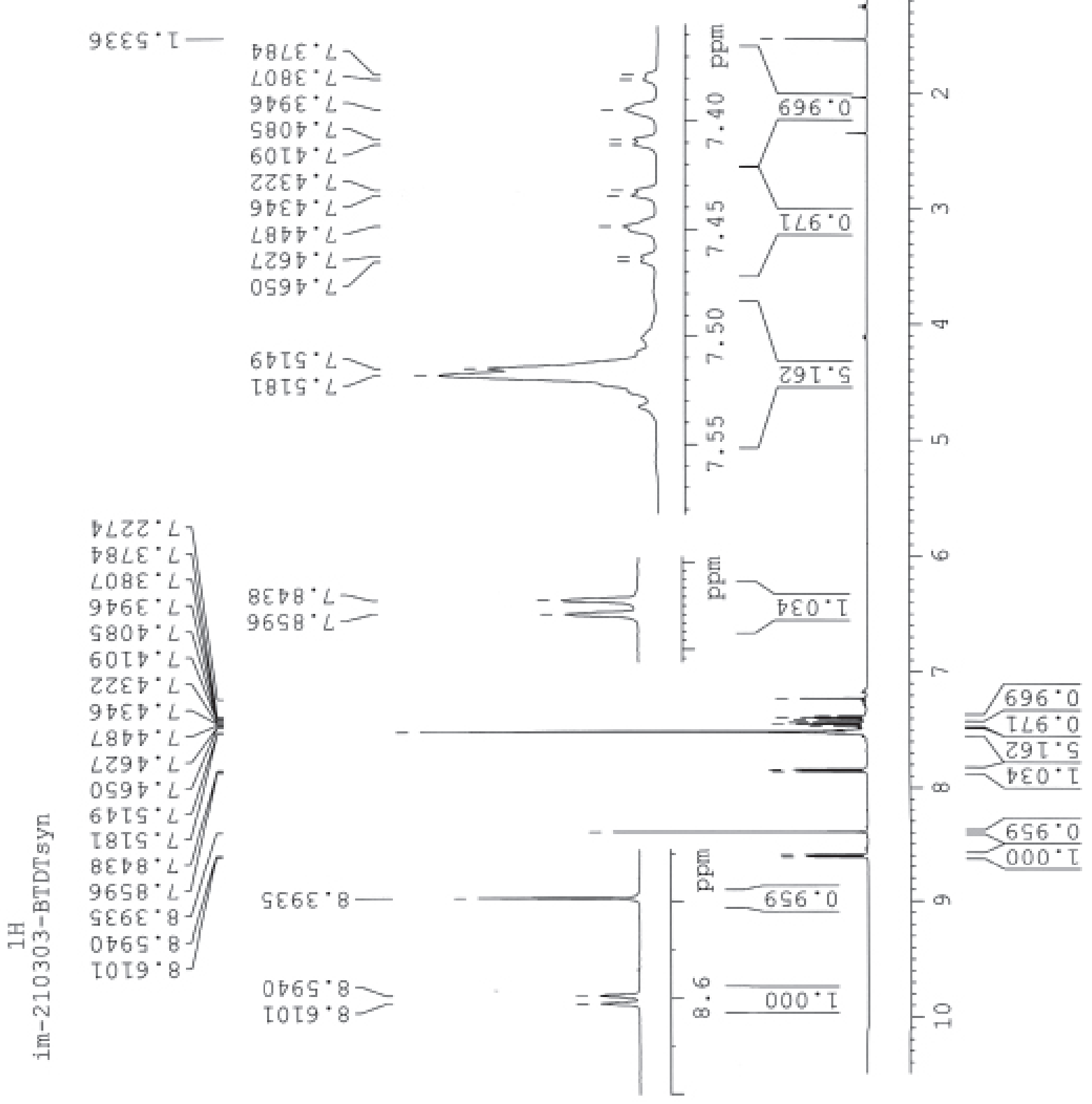


${ }^{13} \mathrm{C}$ NMR Spectrum of Phenyl benzothiophene-3-carbodithioate 1n
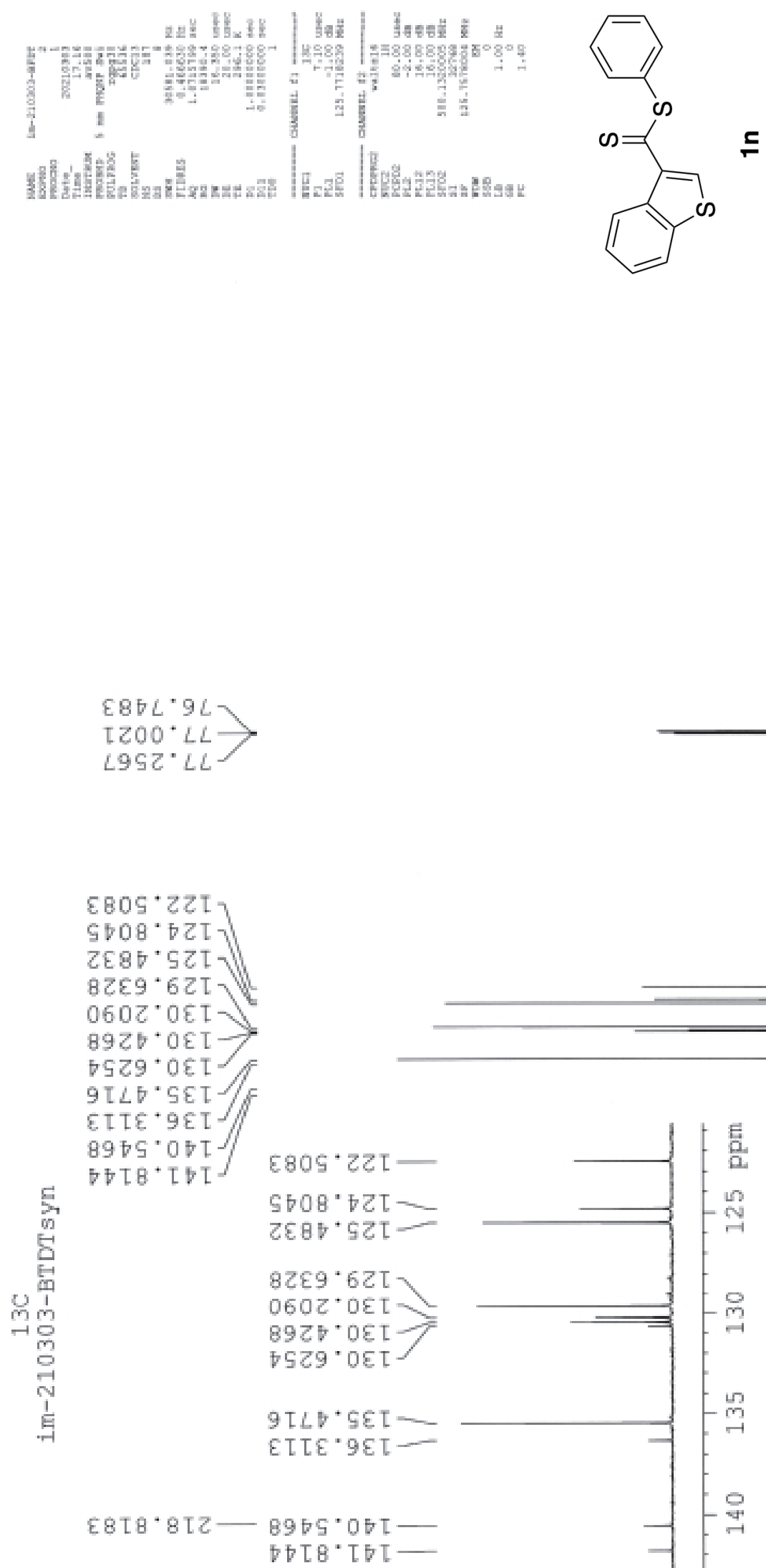

bT $8^{\circ}$ T 6 T 
${ }^{1} \mathrm{H}$ NMR Spectrum of 2-Bromophenyl benzothiophene-3-carbodithioate 10
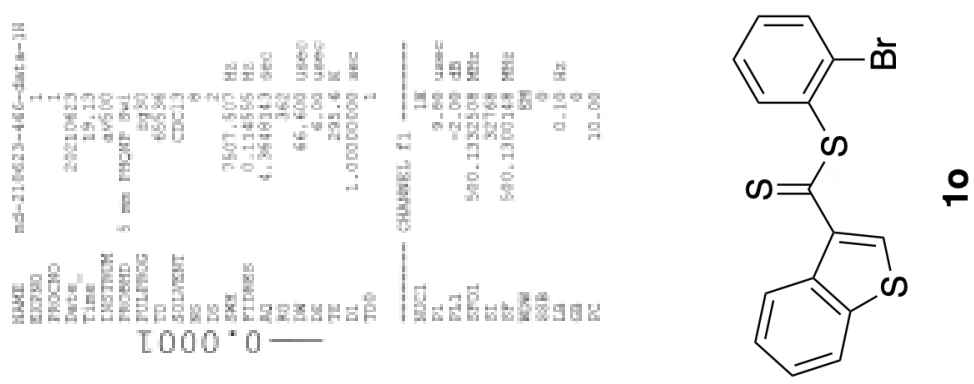

$\operatorname{SESS}^{\circ} \mathrm{T}$
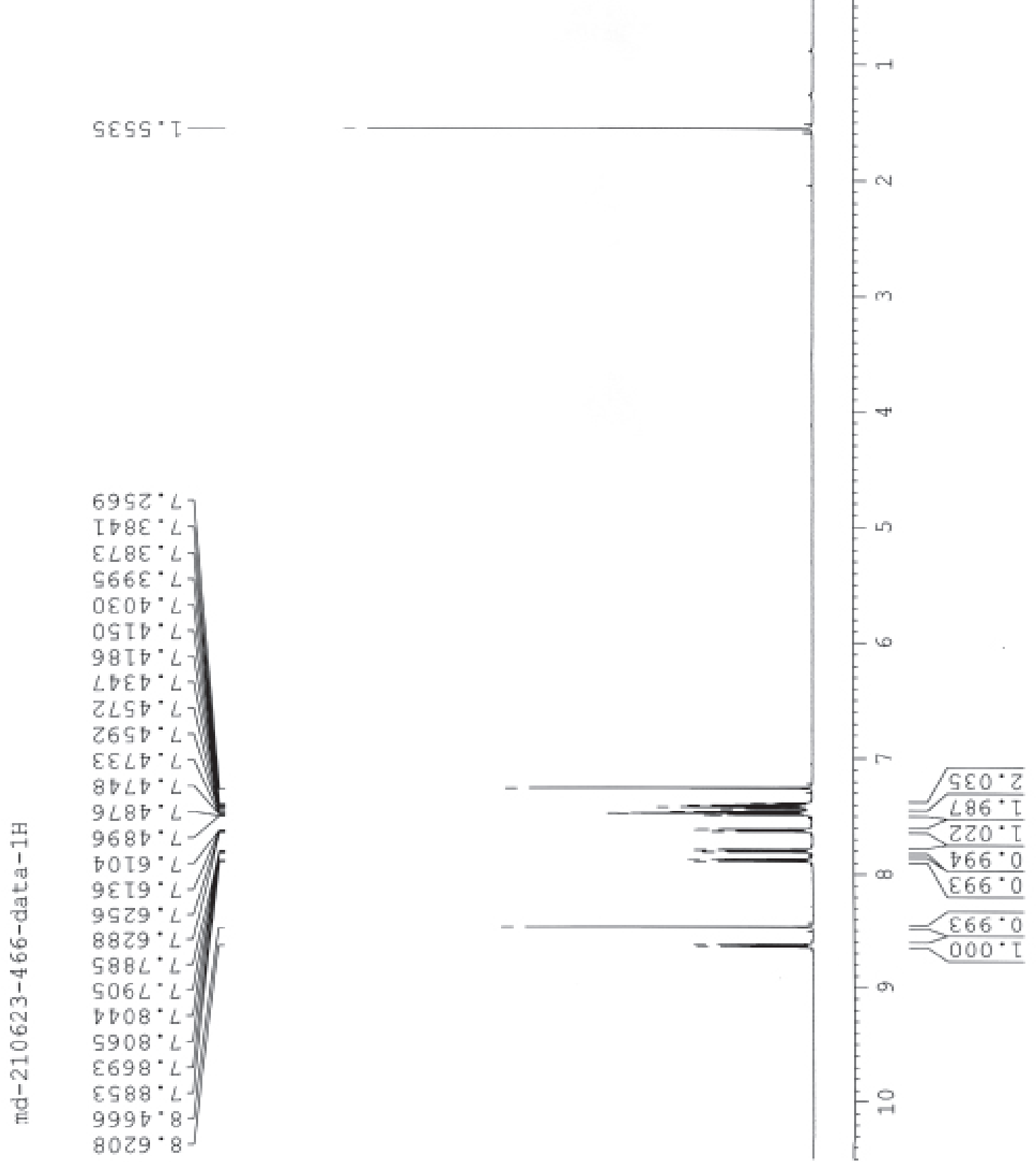
${ }^{13} \mathrm{C}$ NMR Spectrum of 2-Bromophenyl benzothiophene-3-carbodithioate 10
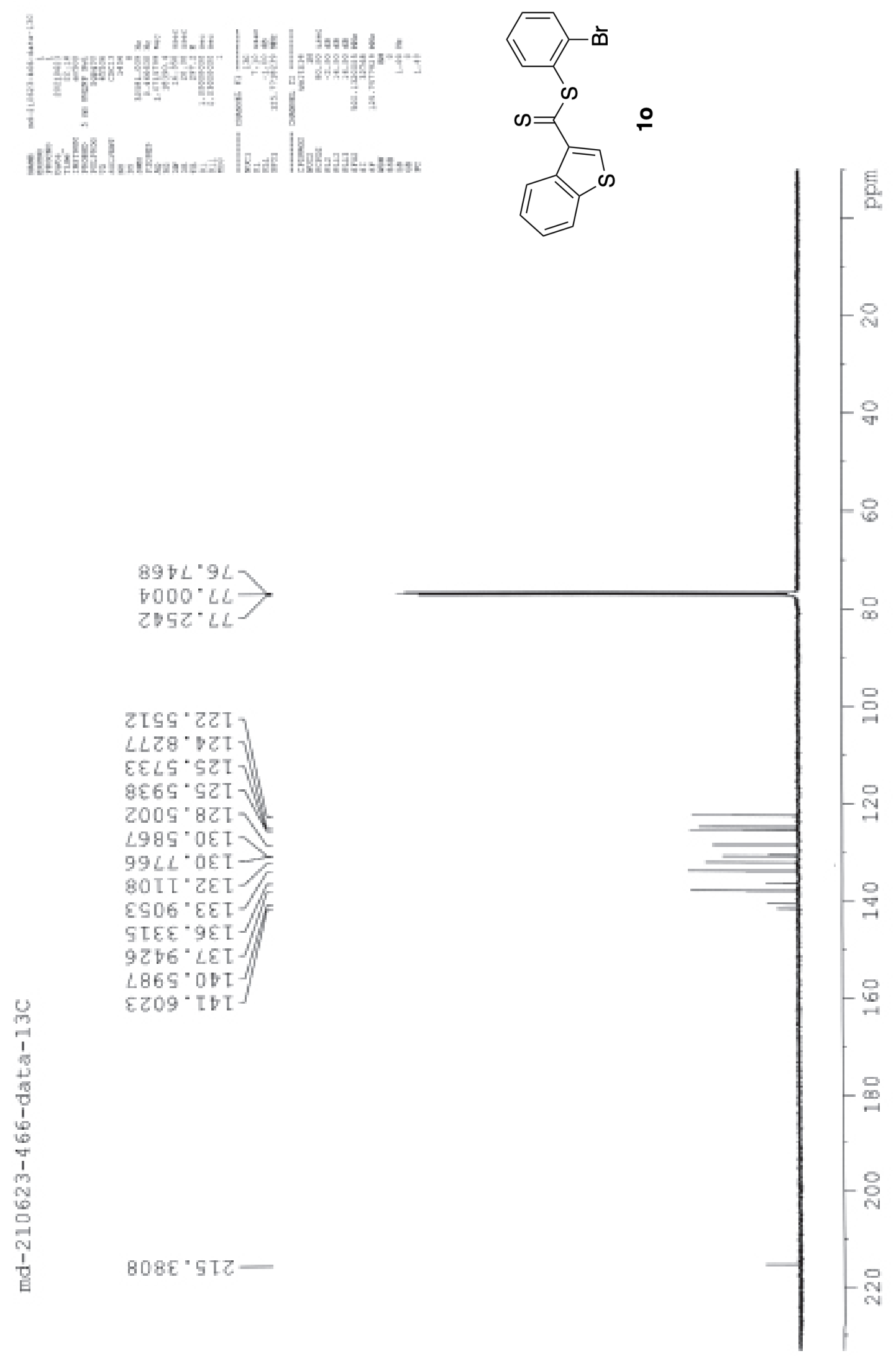
${ }^{1} \mathrm{H}$ NMR Spectrum of 2-Fluoro-3-(phenylsulfanyl)thieno[3,2-b]thiophene 3a
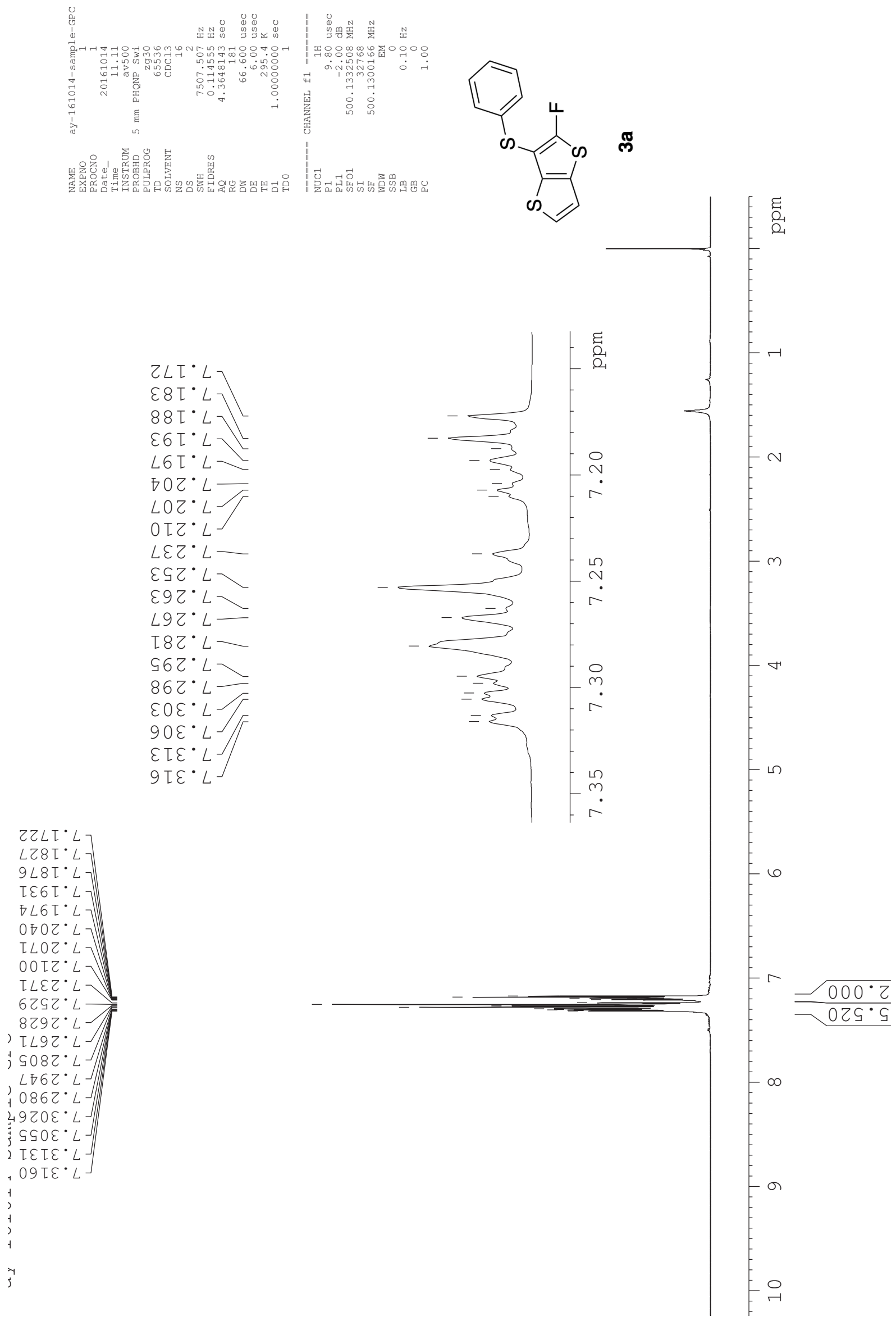

ळ్ల

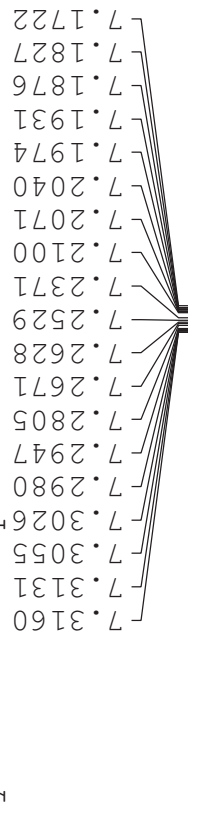


${ }^{13} \mathrm{C}$ NMR Spectrum of 2-Fluoro-3-(phenylsulfanyl)thieno[3,2-b]thiophene 3a

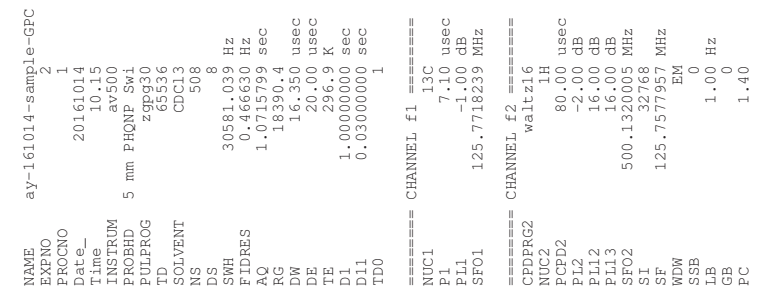

$8928 \cdot \varepsilon 0 \tau \longrightarrow$

乙LE $\sigma^{\circ} \varepsilon O T$

0 ๑ $8 \cdot 6 \tau \tau$

$80[\tau \cdot \bar{\cdot}$ 万I

$9 L \varepsilon[\cdot \nabla Z T$

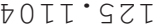

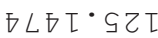

$\varepsilon \varepsilon \nabla L \cdot 92 \mathrm{I}$

$\varepsilon 698^{\circ} 8 Z \mathrm{~T}$

$9 \angle 80^{\circ} 6 Z \mathrm{~T}$

$8 \varepsilon 96^{\circ} \varepsilon \varepsilon T$

ZL6 ${ }^{\circ} \angle \varepsilon T$

8ZSI· $69 I-$

$9 \angle 0 G^{\circ} 99 \mathrm{I}$

$\angle 28 \cdot \varepsilon O T=$

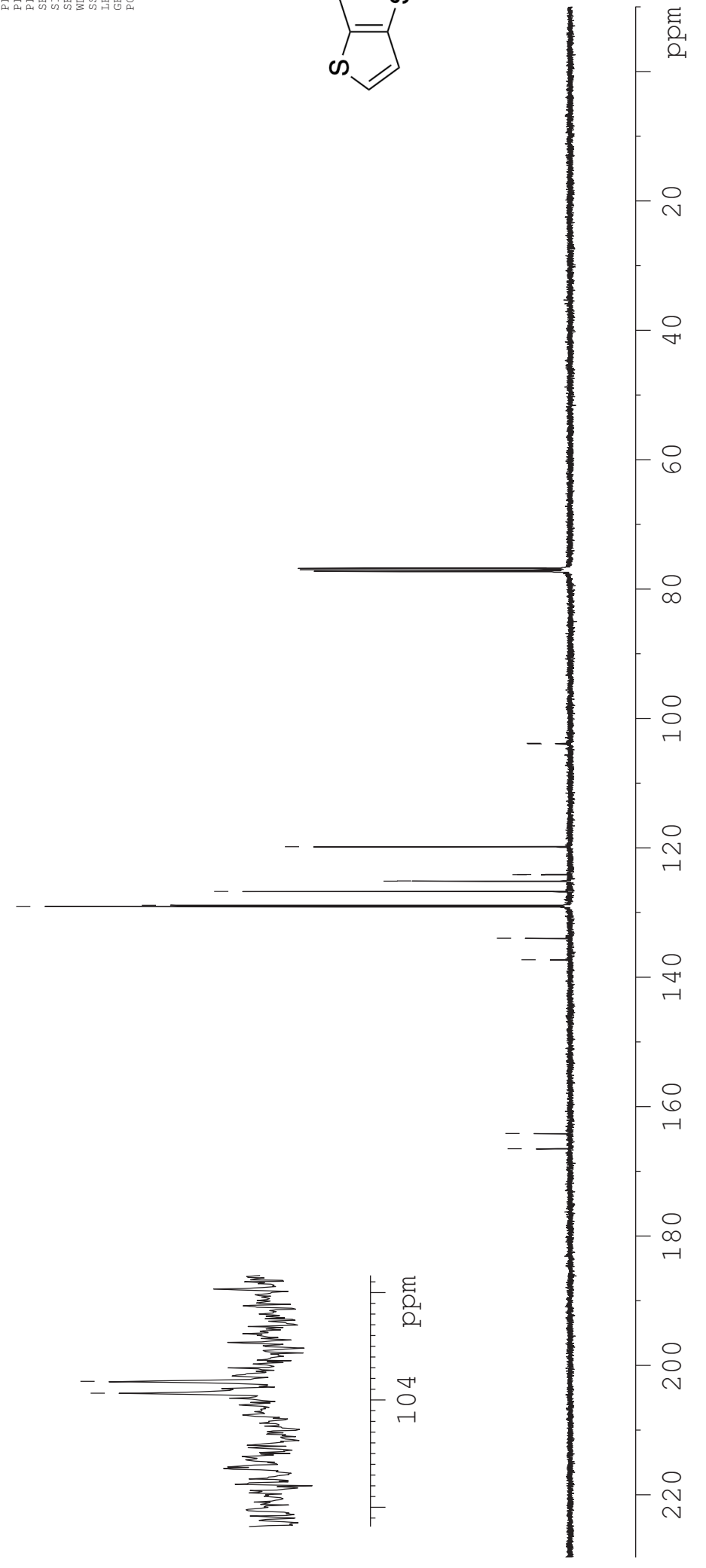


${ }^{19}$ F NMR Spectrum of 2-Fluoro-3-(phenylsulfanyl)thieno[3,2-b]thiophene 3a
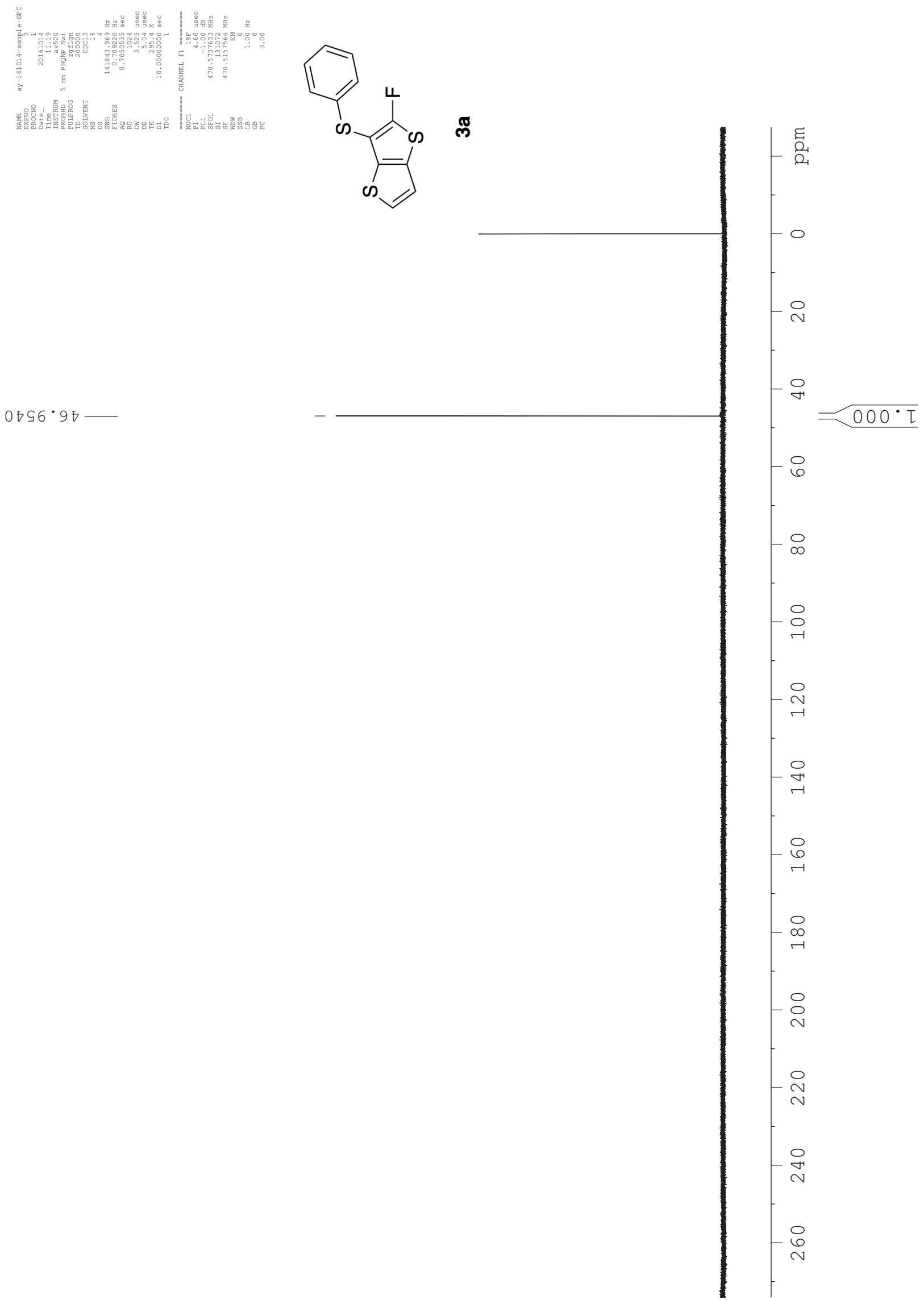

$0796 \cdot 97$ 
${ }^{1}$ H NMR Spectrum of 2-Fluoro-5-methyl-3-(phenylsulfanyl)thieno[3,2-b]thiophene 3b

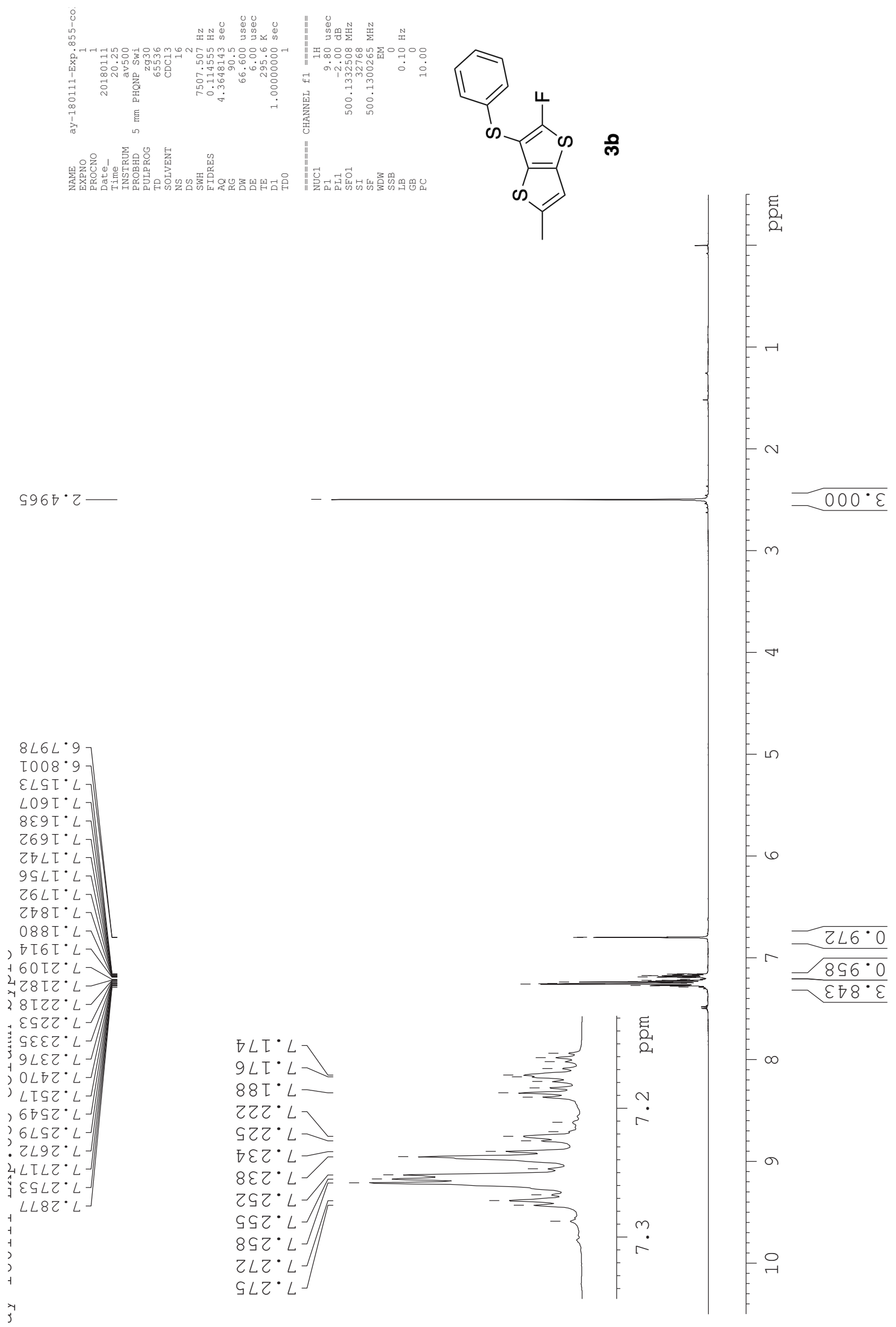


${ }^{13}$ C NMR Spectrum of 2-Fluoro-5-methyl-3-(phenylsulfanyl)thieno[3,2-b]thiophene $\mathbf{3 b}$
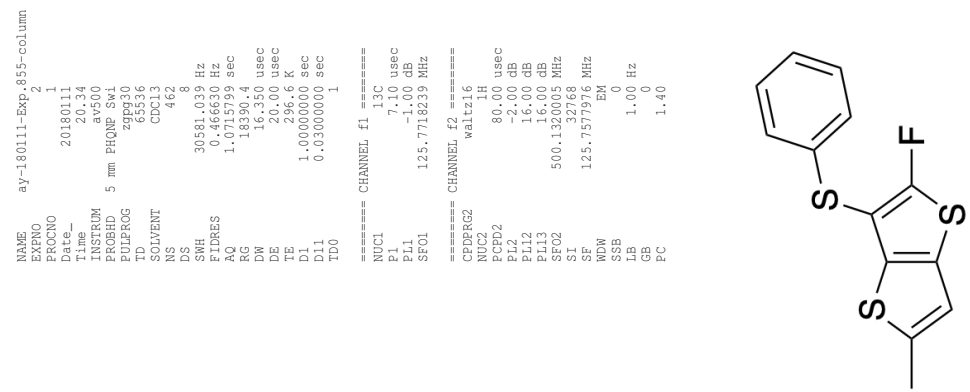

$\varepsilon 020 \cdot 9 I$

б๐ZS $\varepsilon O L$

$6 \varepsilon \varepsilon 9 \cdot \varepsilon 0[>$

$\angle \nabla 8 D^{\circ} \angle I T$

I06 $9^{\circ} \varepsilon Z T$

L8โ9.हटा

$6889^{\circ} \cdot 9 Z I$

$20 \angle 9 \cdot 8 Z I$

ILEO 6 С T -

LEOZ $\nabla \varepsilon I-$

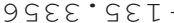

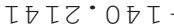

пโSZ・OェT-

$\varepsilon \angle 6 I \cdot \varepsilon 9 T-$

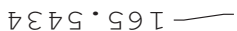

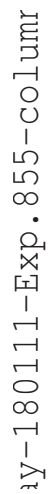
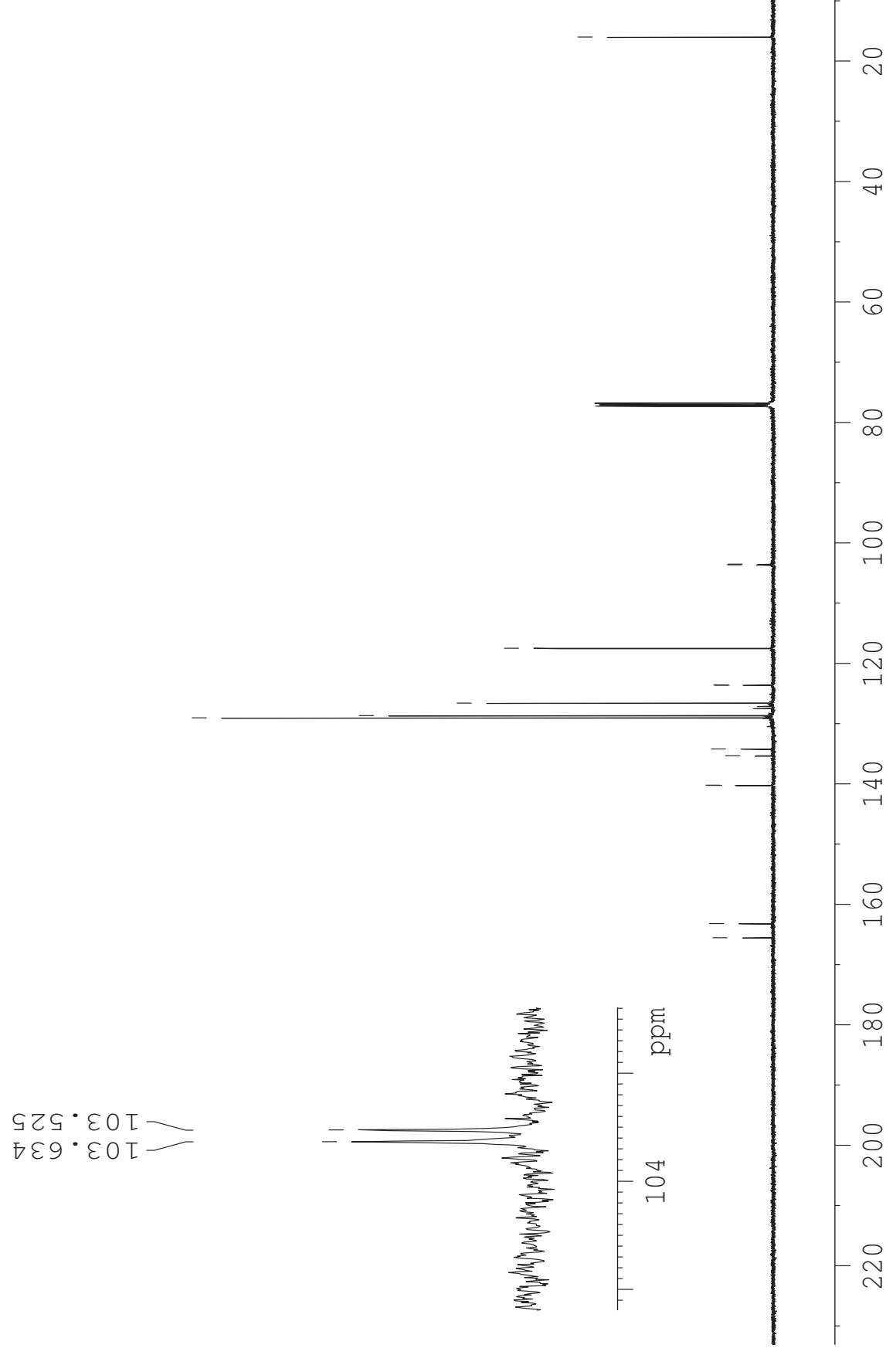
${ }^{19}$ F NMR Spectrum of 2-Fluoro-5-methyl-3-(phenylsulfanyl)thieno[3,2-b]thiophene 3b
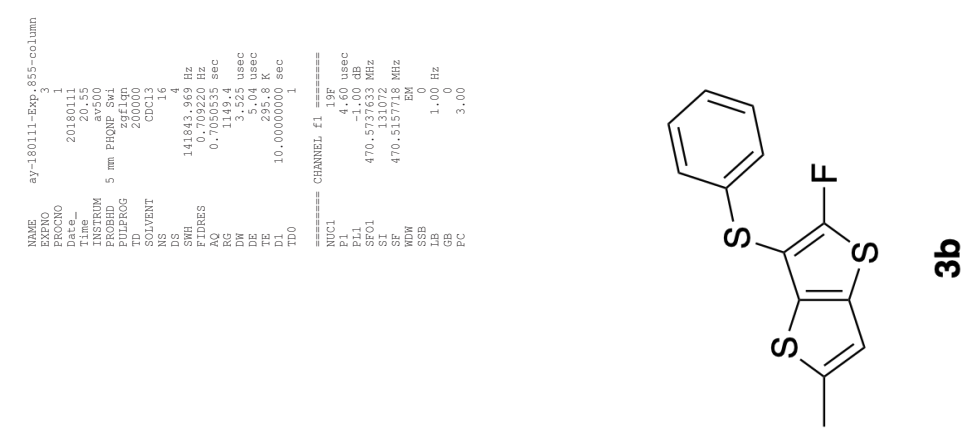

$8 S \tau \nabla \cdot \varepsilon \overline{-}$ 
${ }^{1} \mathrm{H}$ NMR Spectrum of 2-Fluoro-5-phenyl-3-(phenylsulfanyl)thieno[3,2-b]thiophene 3c

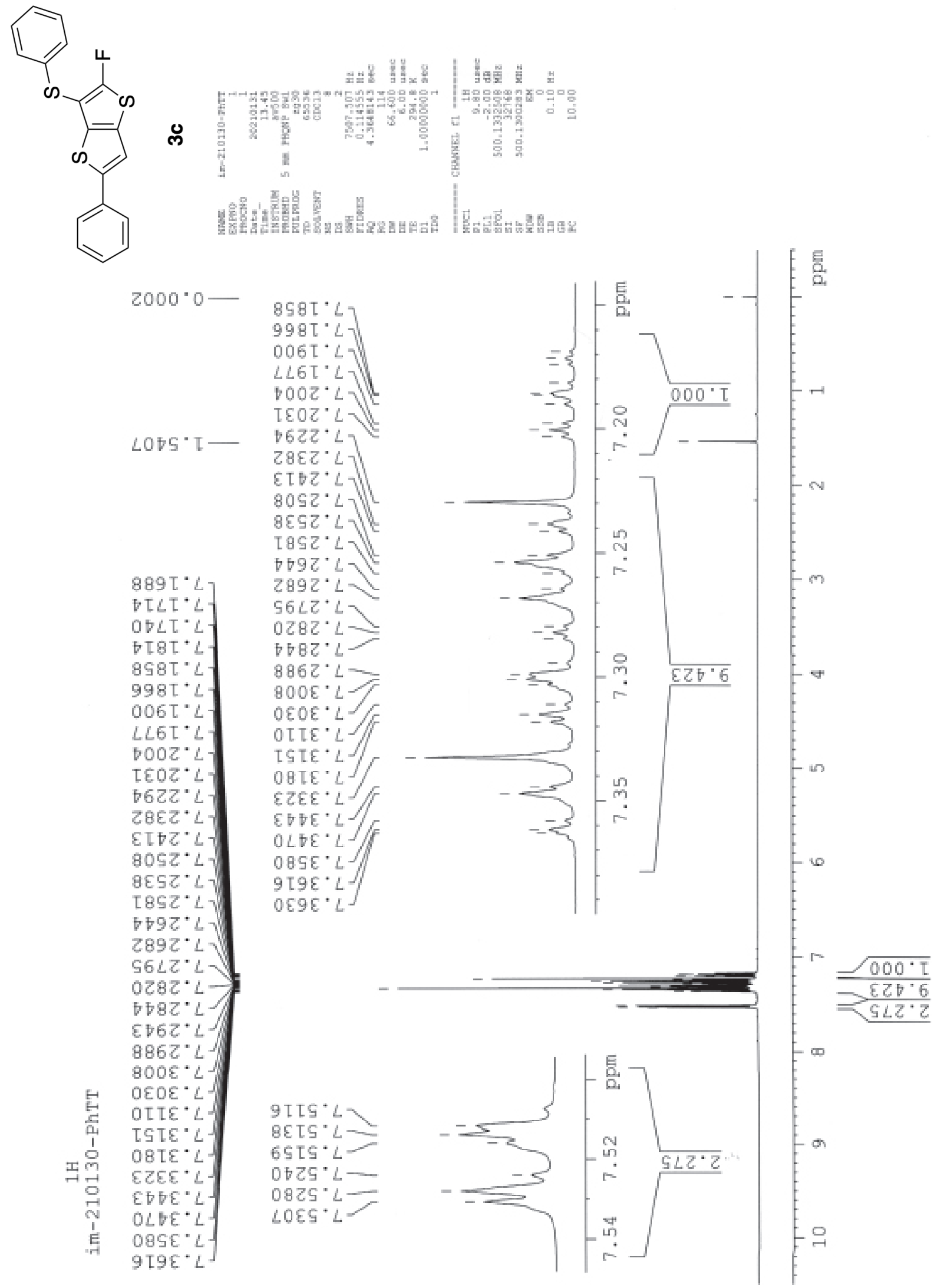


${ }^{13} \mathrm{C}$ NMR Spectrum of 2-Fluoro-5-phenyl-3-(phenylsulfanyl)thieno[3,2-b]thiophene 3c
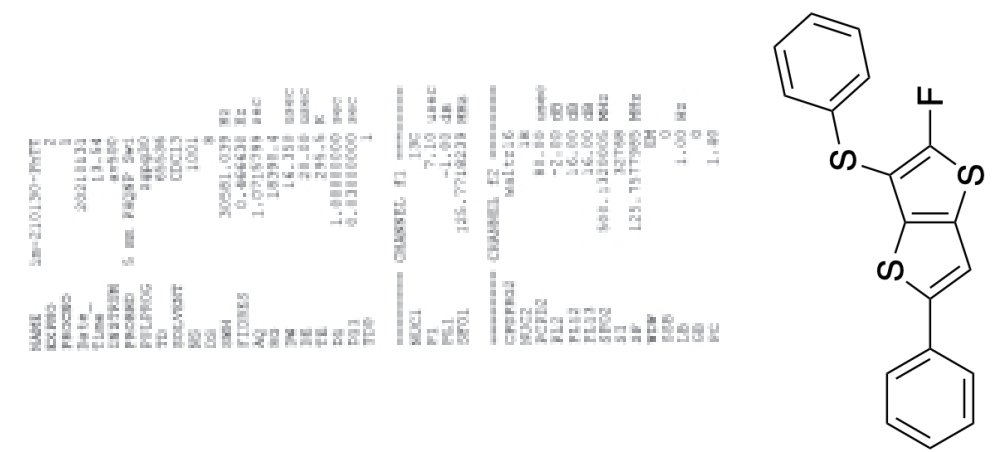

@

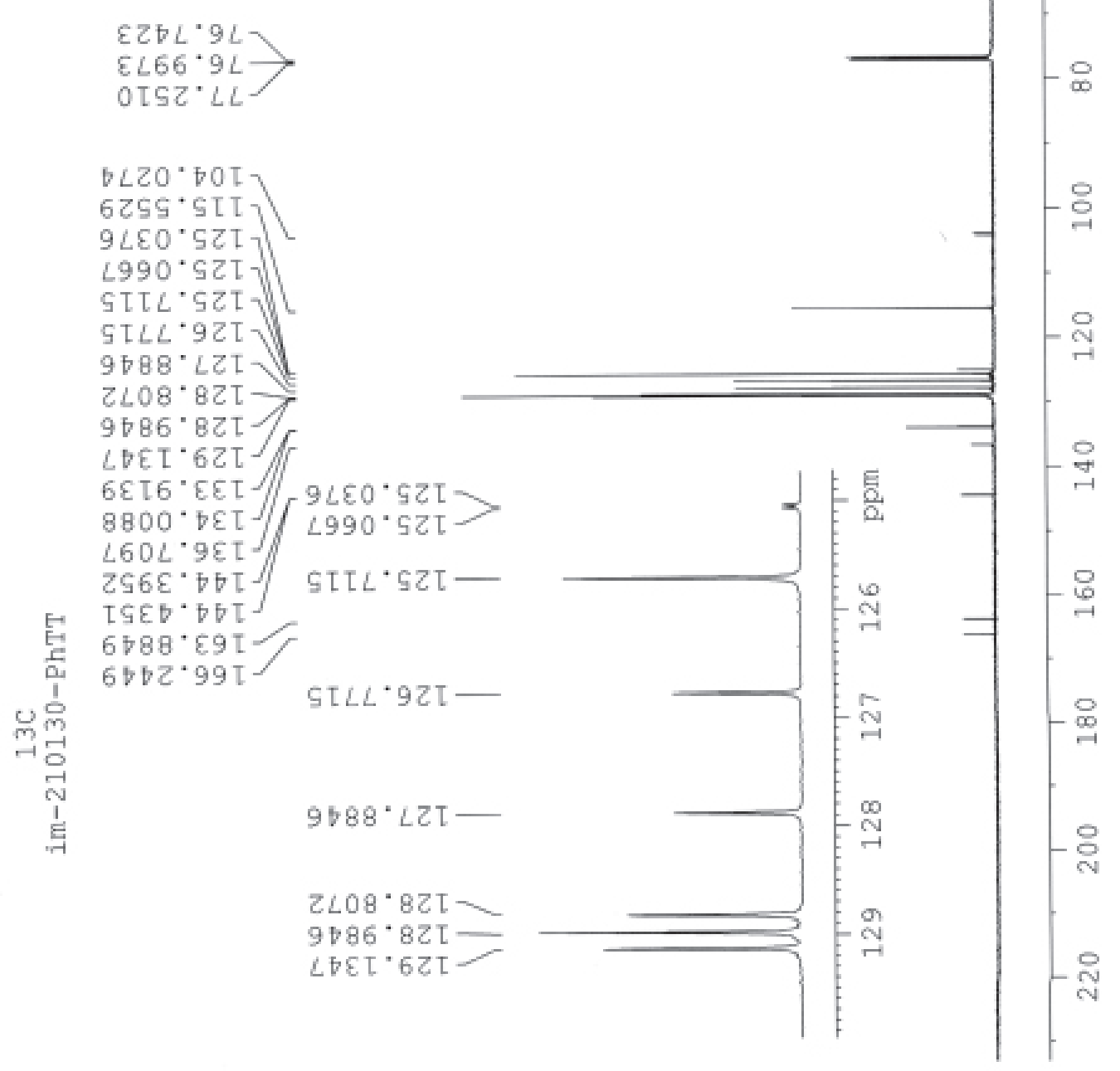


${ }^{19}$ F NMR Spectrum of 2-Fluoro-5-phenyl-3-(phenylsulfanyl)thieno[3,2-b]thiophene 3c
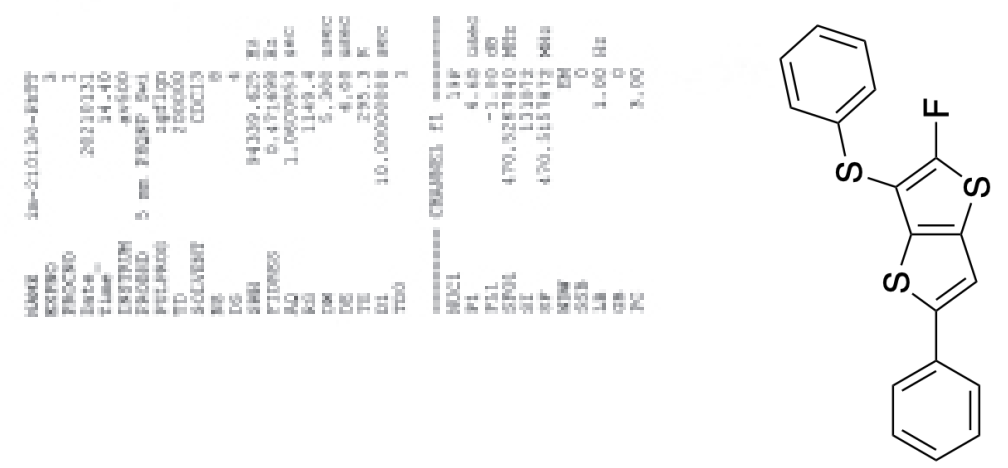

迎

$0000^{\circ} 0$

$2996^{\circ} 56-$
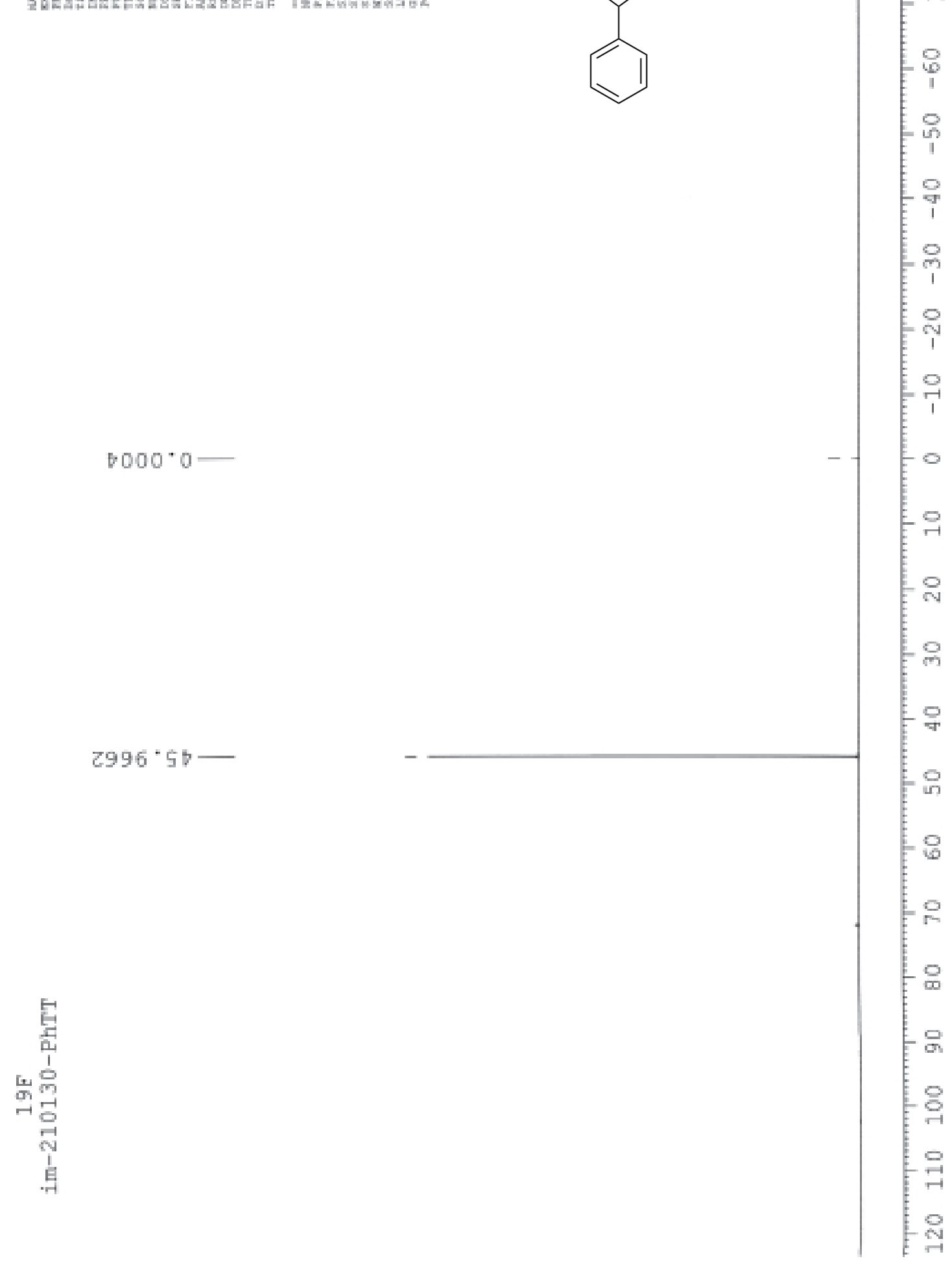
${ }^{1} \mathrm{H}$ NMR Spectrum of 2-Fluoro-3-(phenylsulfanyl)thieno[3,2- $\left.b\right][f]$ benzothiophene 3d
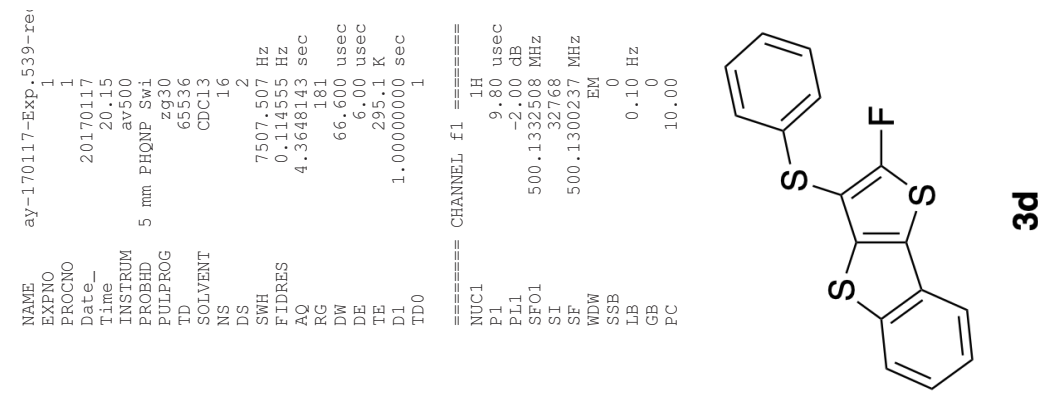

ర్ల
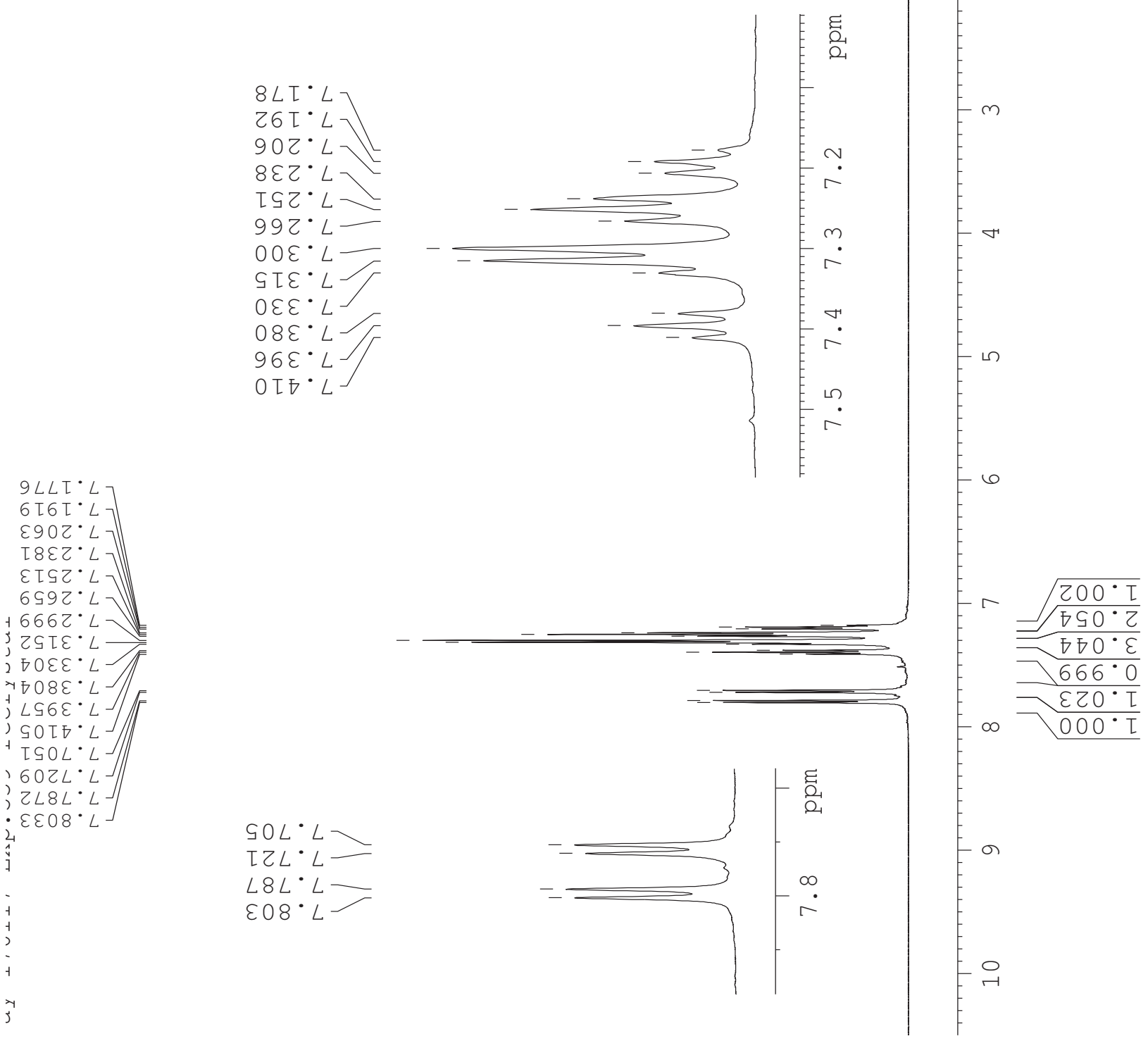
${ }^{13} \mathrm{C}$ NMR Spectrum of 2-Fluoro-3-(phenylsulfanyl)thieno[3,2-b][f]benzothiophene 3d
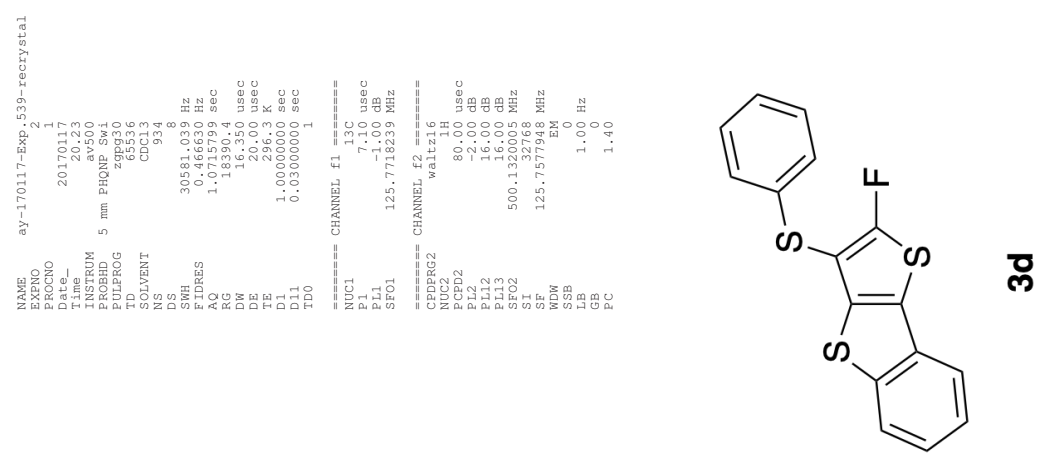

$6 \nabla 00 \cdot S 0 T$

乙ELI. $50 I$

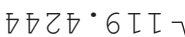

$8 \nabla S \sigma \cdot 6[\tau]$

$0766^{\circ} 6 \tau[$

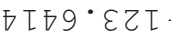

LS92・五乙T

$\varepsilon 6 \varsigma 6^{\circ} \nabla Z T$

$6978 \cdot 92 \tau$

$08 \angle 8 \cdot 8 Z \mathrm{I}$

$899 \tau \cdot 62 \tau]$

与 $6 \tau$ เ $\varepsilon \varepsilon \tau /$

$7086^{\circ} \varepsilon \varepsilon[\rfloor$

$6680^{\circ}$ LEI $]$

$0[\angle 8 \cdot 6 \varepsilon[]$

$6268^{\circ} 6 \varepsilon \mathrm{I}$

60 TS・ 79 L

E†88.99 I

+
0
01
4
0
0
4
1
1
0
$m$
0
$\dot{0}$
0
$x$
0

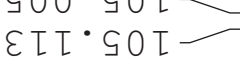

$S 00 \cdot S O L=$
$\varepsilon I T \cdot S O L=$

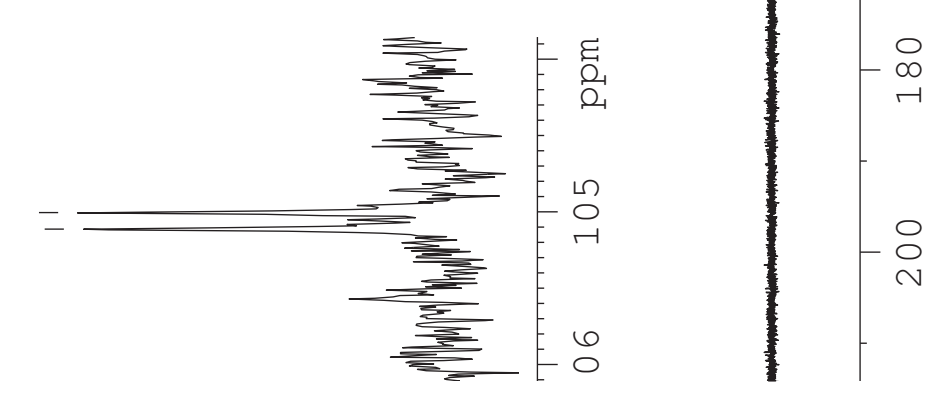


${ }^{19}$ F NMR Spectrum of 2-Fluoro-3-(phenylsulfanyl)thieno[3,2-b][f]benzothiophene 3d
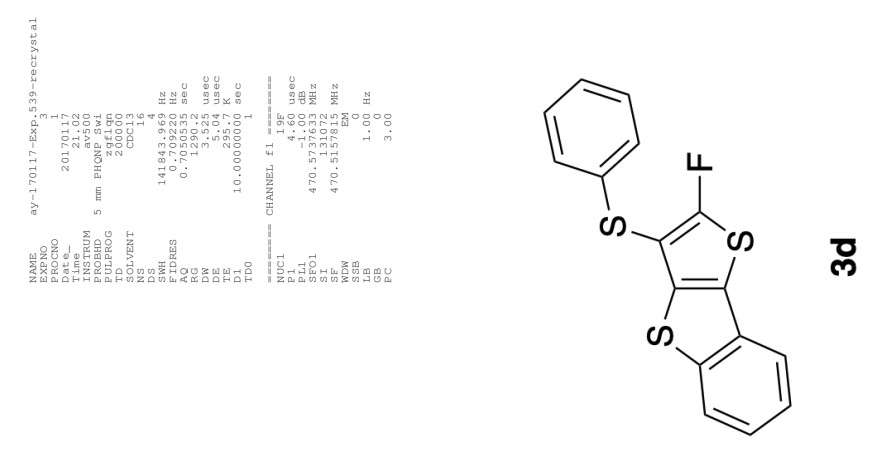

$8 I I Z \cdot 87-$

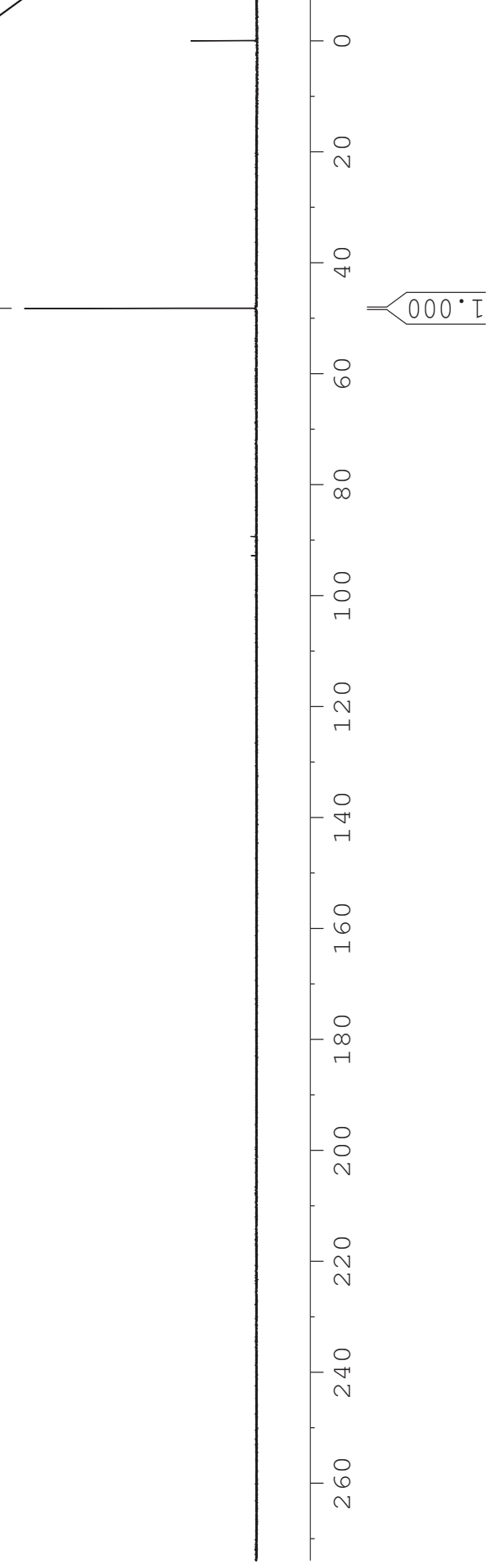


${ }^{1}$ H NMR Spectrum of 2-Fluoro-7-methyl-3-(phenylsulfanyl)benzo[b]thieno[2,3- $\left.d\right]$ thiophene $\mathbf{3 e}$

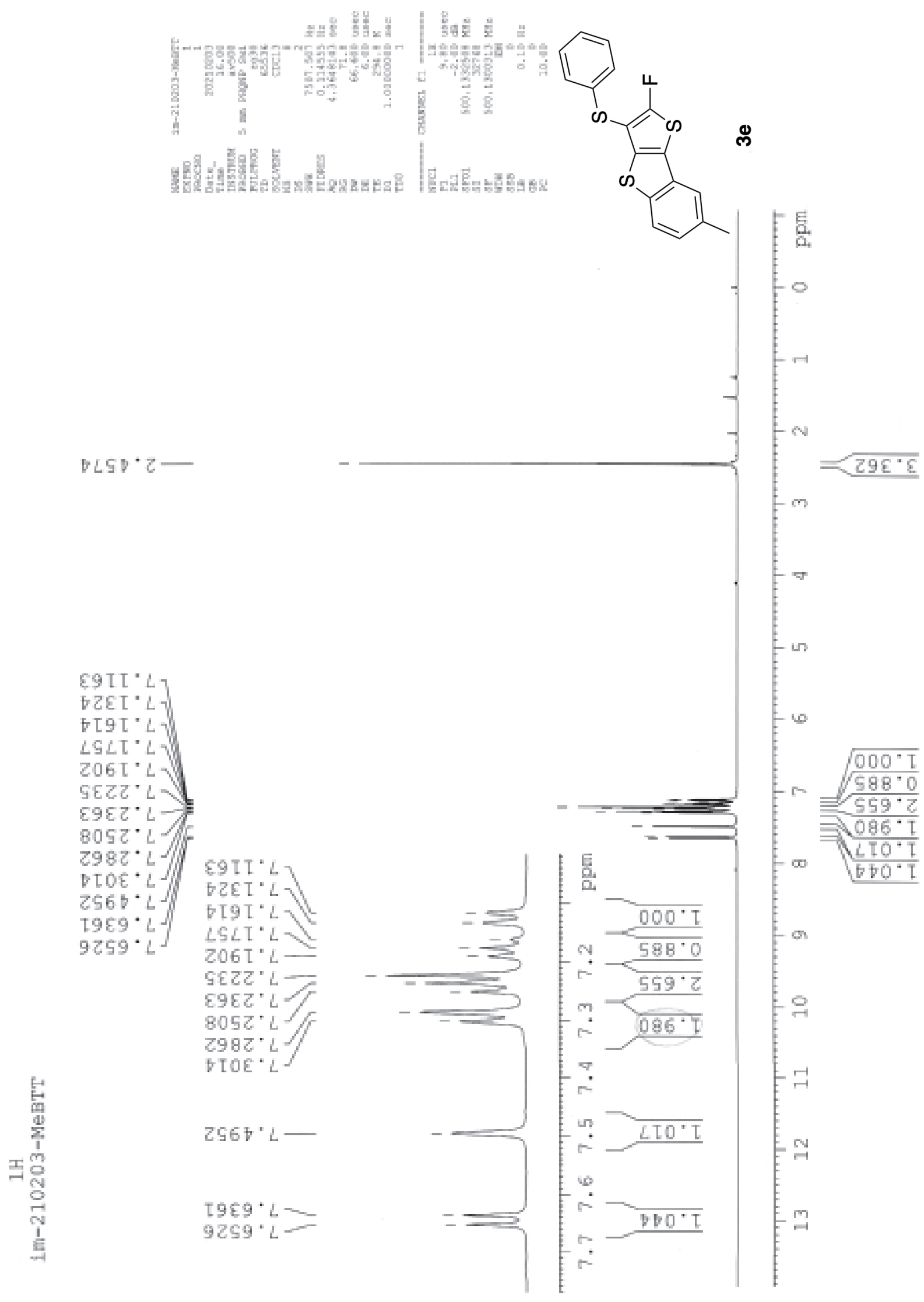


${ }^{13}$ C NMR Spectrum of 2-Fluoro-7-methyl-3-(phenylsulfanyl)benzo[b]thieno[2,3- $\left.d\right]$ thiophene 3e

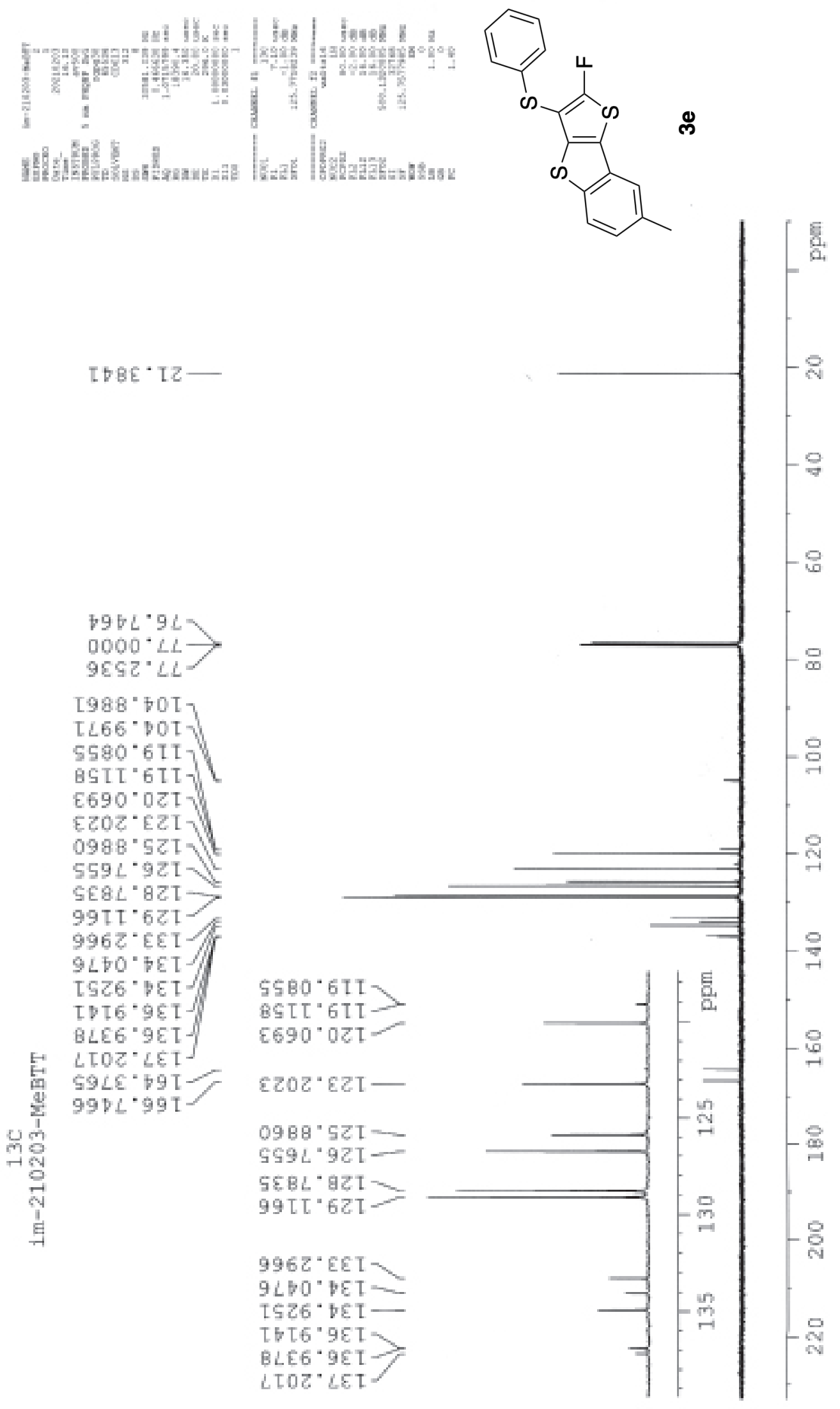


${ }^{19}$ F NMR Spectrum of 2-Fluoro-7-methyl-3-(phenylsulfanyl)benzo[b]thieno[2,3-d] thiophene 3e
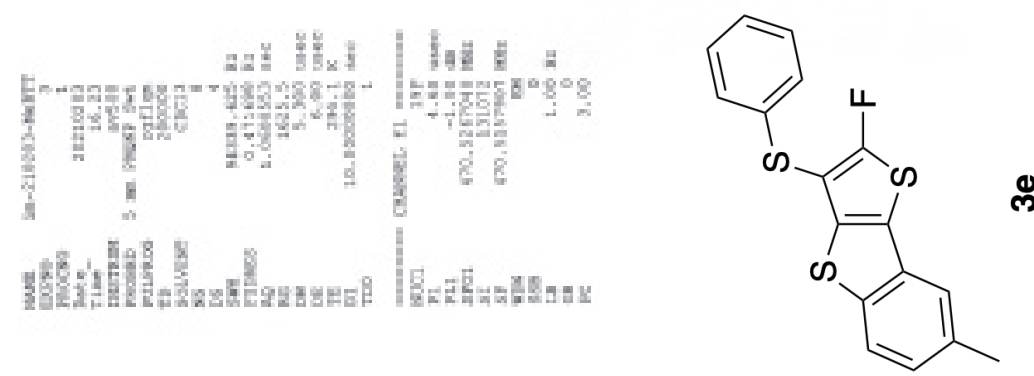

$6000^{\circ} 0-$

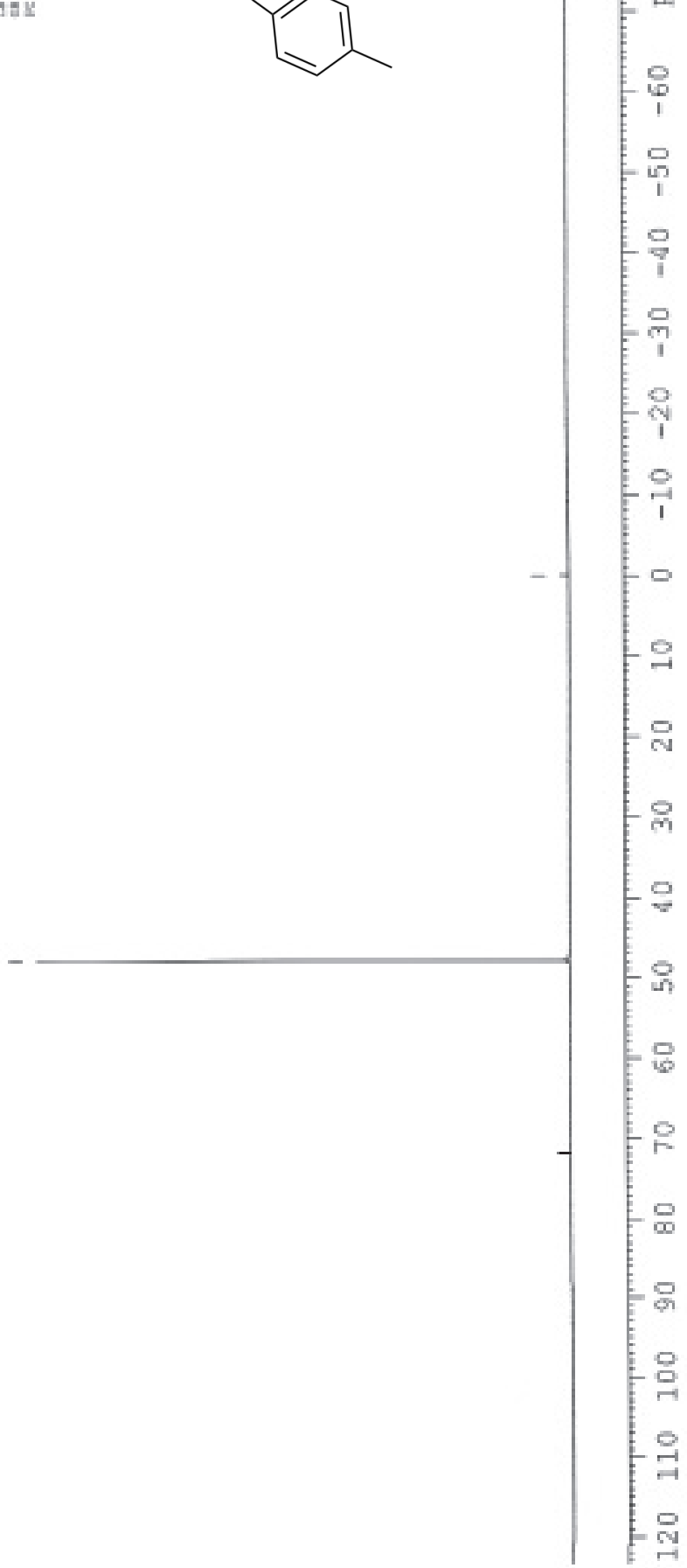

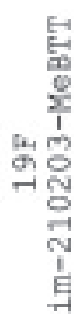

$5598^{\circ} \angle F-$

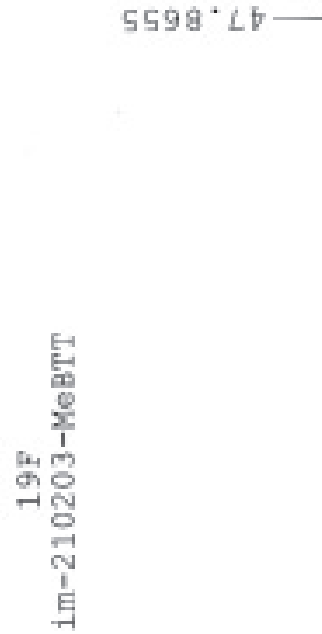


${ }^{1} \mathrm{H}$ NMR Spectrum of 7-Chloro-2-fluoro-3-(phenylsulfanyl)benzo[b]thieno[2,3-d]thiophene 3f<smiles>Cc1oc2c(oc3ccc(O)cc32)c1Oc1ccccc1</smiles>

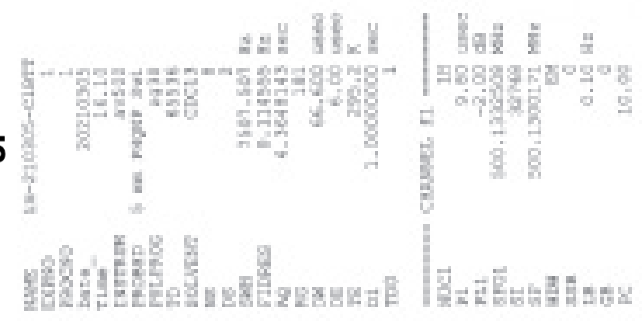

$2000 \cdot 0$
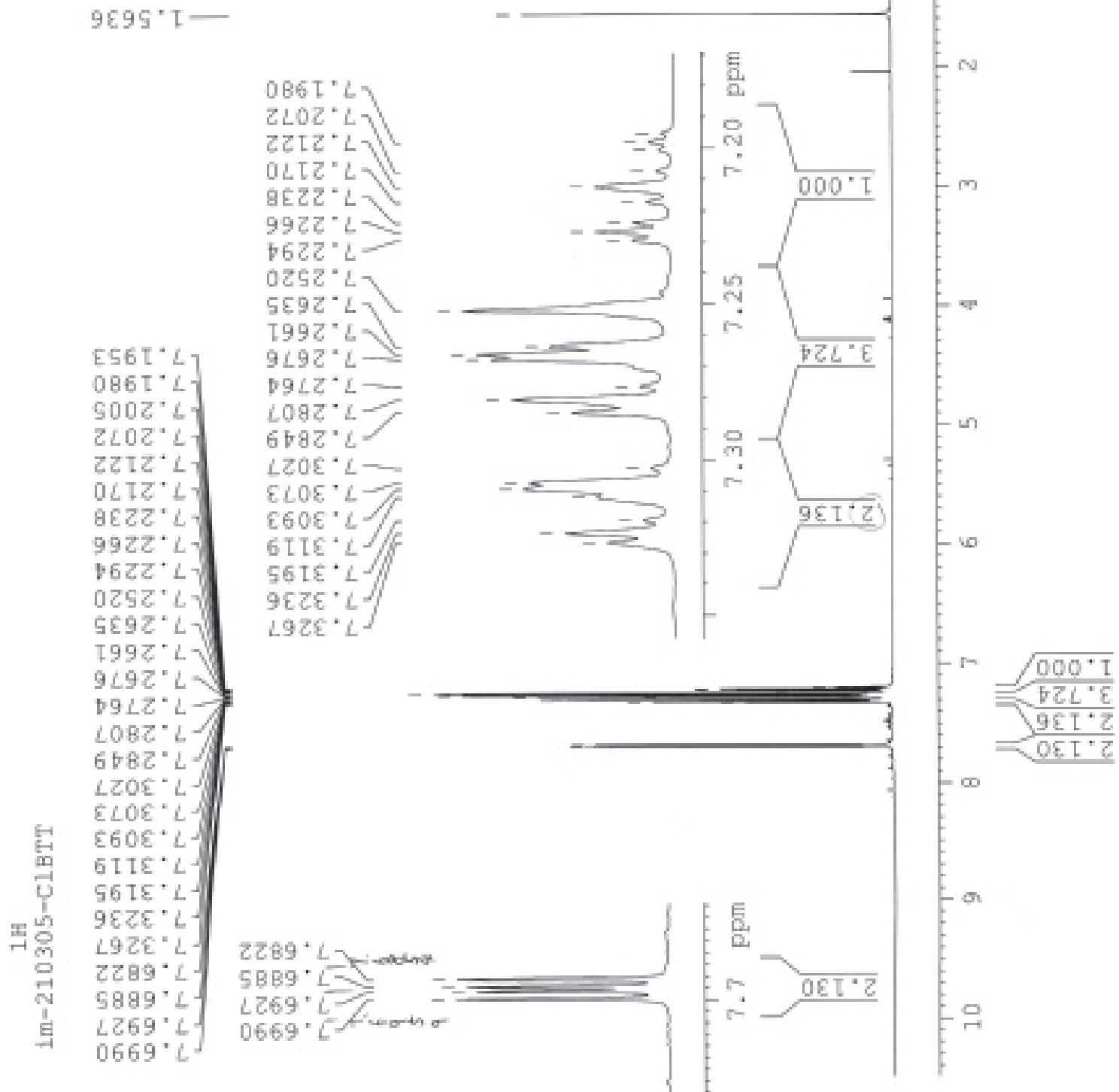
${ }^{13} \mathrm{C}$ NMR Spectrum of 7-Chloro-2-fluoro-3-(phenylsulfanyl)benzo[b]thieno[2,3- $\left.d\right]$ thiophene $\mathbf{3 f}$
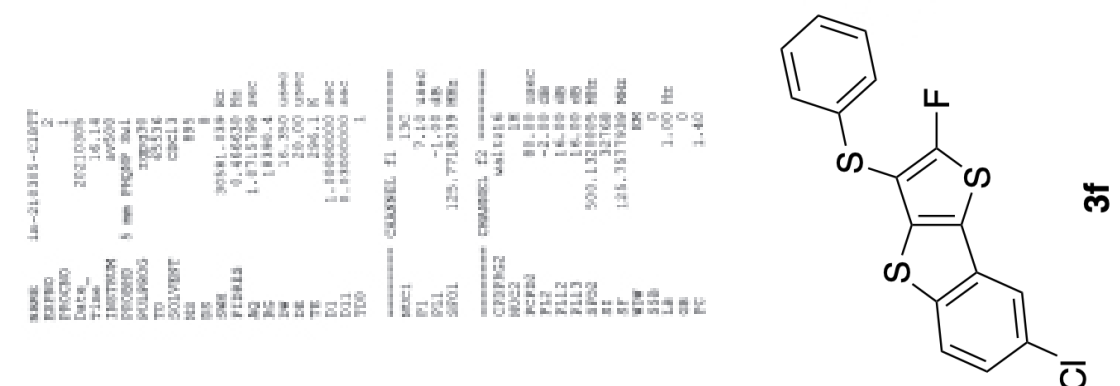

I $60 L \cdot 9 L$

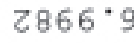

$8 \mathrm{tS} \mathrm{C}^{\circ} \mathrm{L}$

9LBE $\mathrm{GOT}$.

88S . $^{\circ} 0 \mathrm{~L}$

9 โG5.8 8 I ]

$\angle 985^{\circ} 8 \mathrm{IT}$.

จ $\tau L L^{*} 6 \tau \tau$,

$8 \nabla Z S^{\circ} \nabla Z \tau$,

$96 \angle S^{\circ}$ DZT.

S88 ${ }^{\circ} \nabla 2 \mathrm{~T}$

$6750^{\circ} \mathrm{LZI}$

LGZT'6ZT

$89 \tau Z^{*} 6 z t$

$\varepsilon \nabla 6 Z^{*}$ TE T

SLT

$6629^{\circ} \varepsilon \varepsilon[/$

$\left.8 \nabla \varsigma{ }^{*} b \varepsilon \tau\right]$

$\varepsilon 666^{\circ}$ LEL

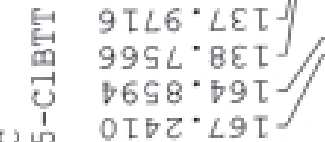

9โรS.8IT

$\angle 985^{\circ} 8$ IT

bIEL.6IT-

8चZS"

$96 \angle S^{\circ} \cdot b Z L$

与88.

$6 \pm 50^{\circ} \angle Z T-$

$\angle S Z L \cdot 6 Z I$

$8 \mathrm{STZ} \cdot 6 \mathrm{ZT}$

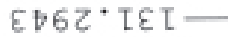

$\varsigma L \tau 9^{\circ} \varepsilon \varepsilon \tau$

ฤ $29^{\circ} \varepsilon \varepsilon \tau$

$8 \sqcup ५{ }^{\circ} \sqcup \varepsilon \tau$

$\varepsilon 676^{\circ} \mathrm{LET}$

$9 \tau \angle 6^{\circ} \angle E T$

$995 L^{\circ} 8 \varepsilon \tau$

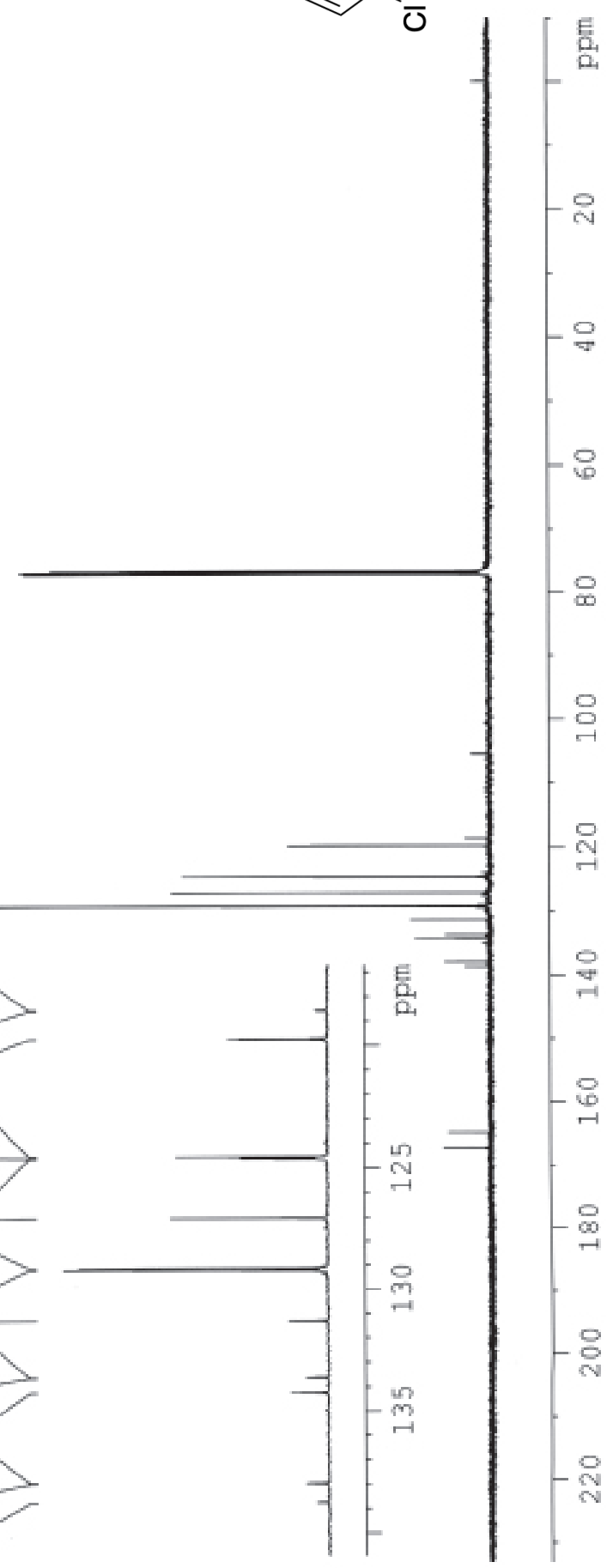


${ }^{19}$ F NMR Spectrum of 7-Chloro-2-fluoro-3-(phenylsulfanyl)benzo[b]thieno[2,3- $\left.d\right]$ thiophene $\mathbf{3 f}$
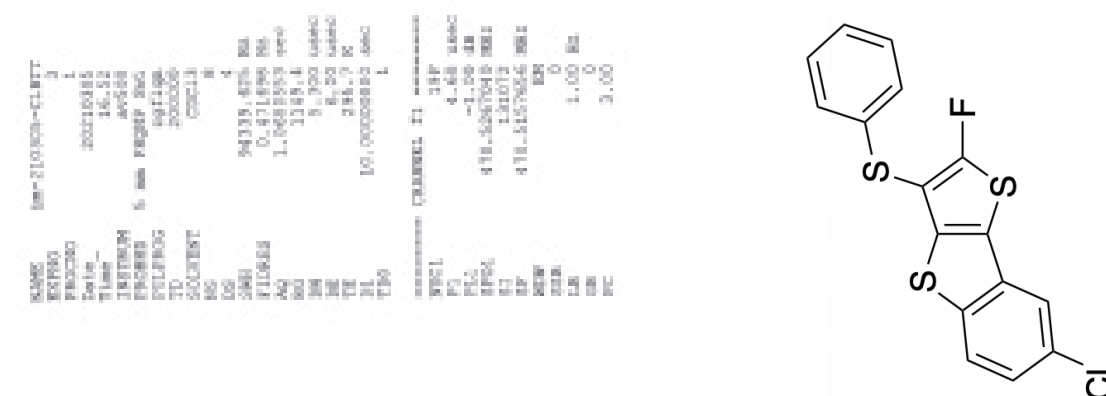

$0000^{\circ} 0-$

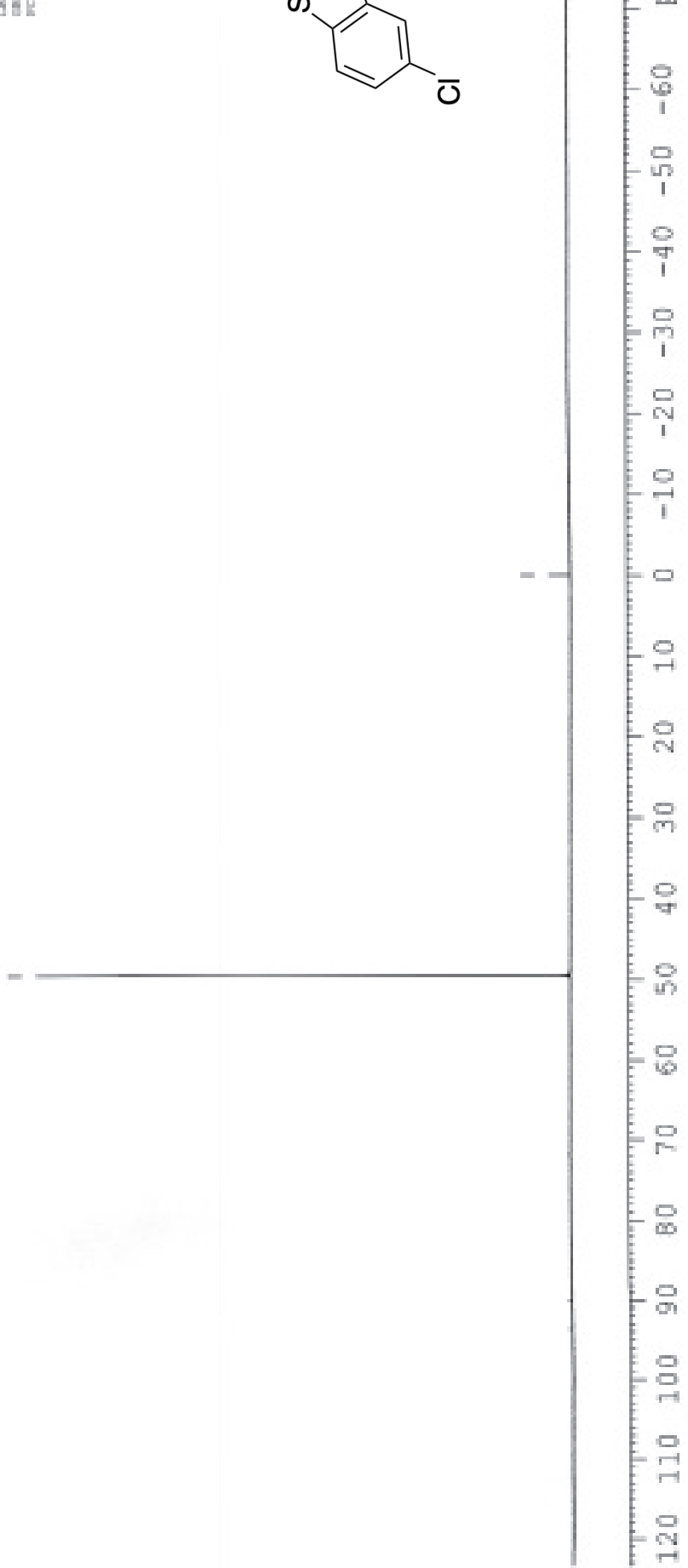

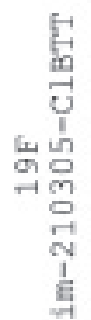

$\cos 5^{\circ} 6 t^{\circ}-$

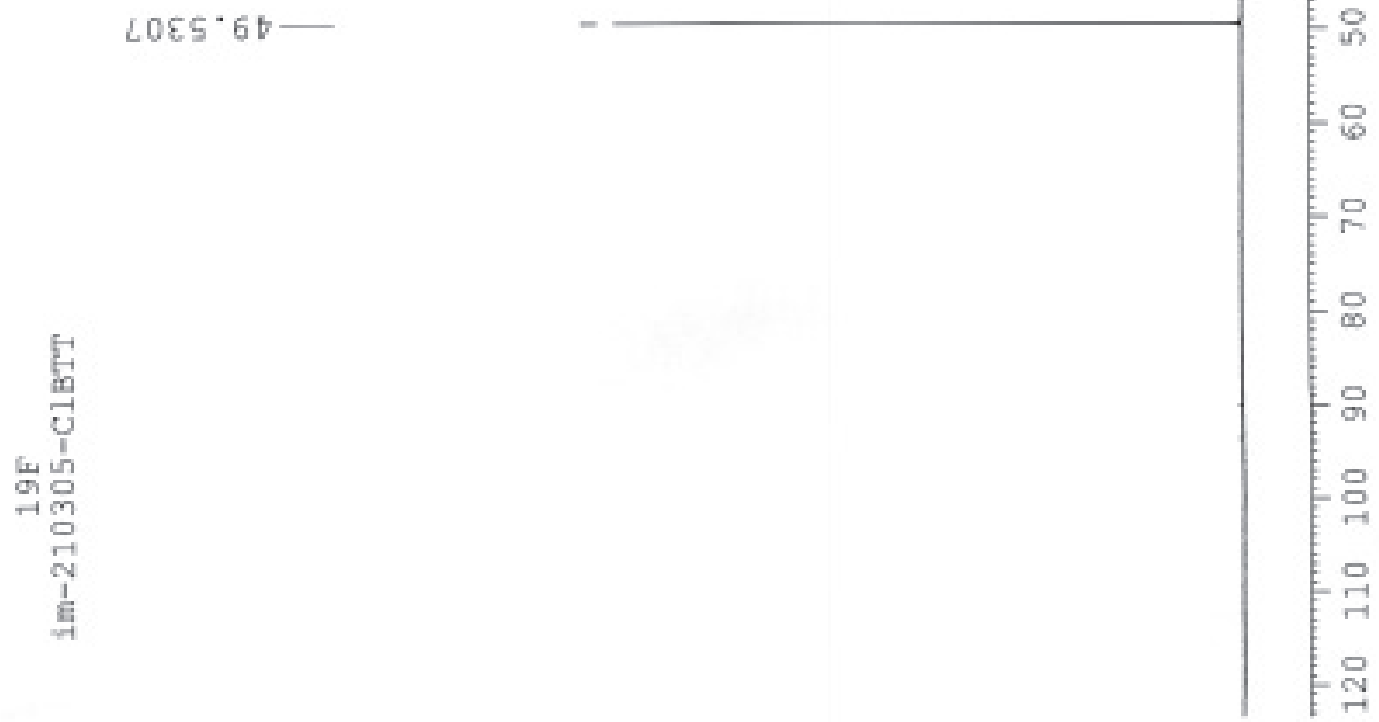




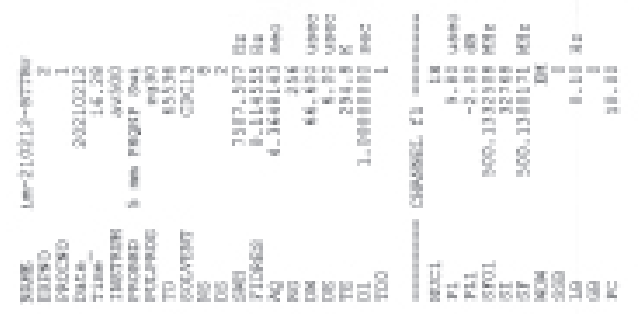<smiles>O=Cc1oc2c(oc3ccccc32)c1Oc1ccccc1Cl</smiles>

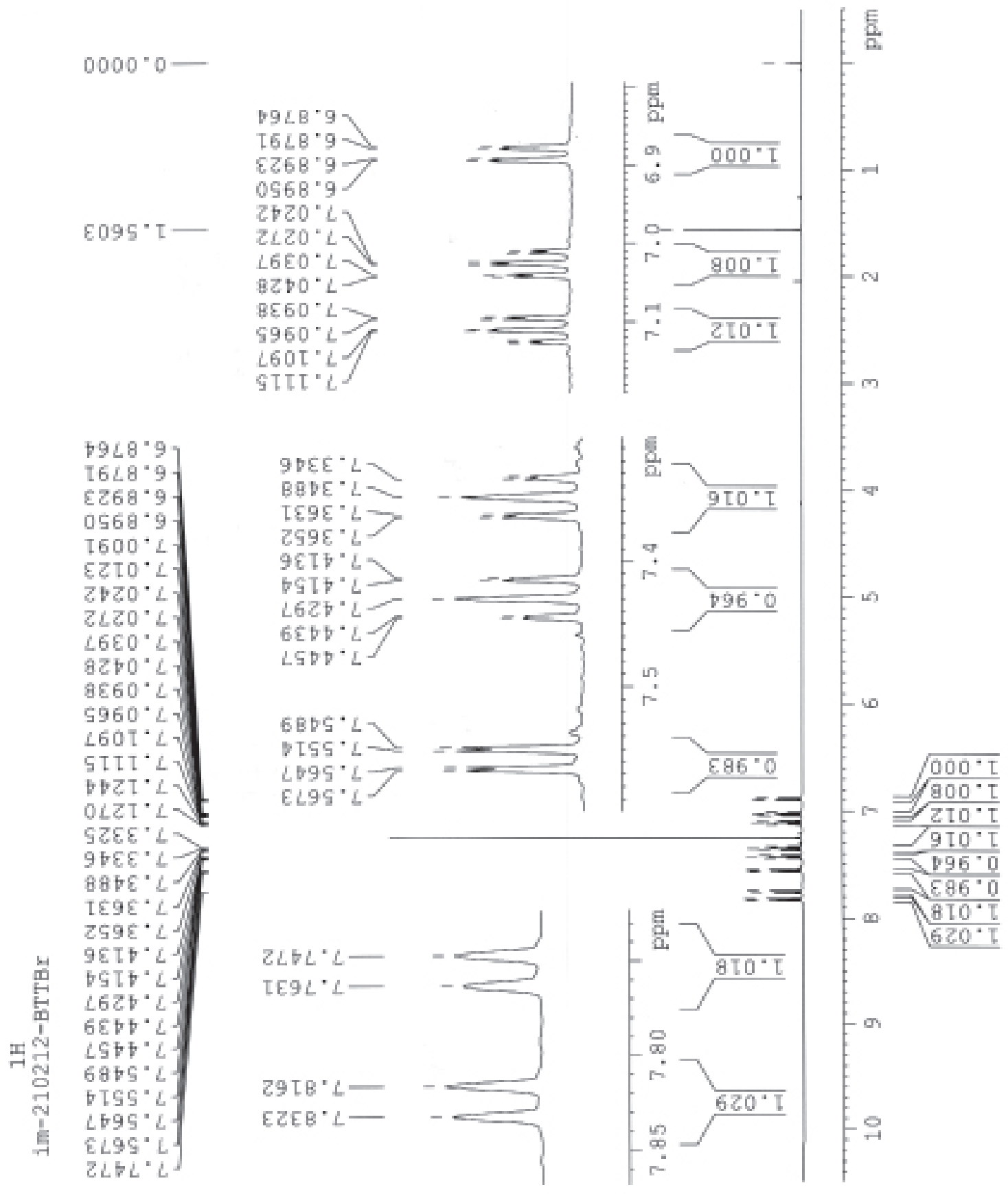


${ }^{13} \mathrm{C}$ NMR Spectrum of 3-[(2-Bromophenyl)sulfanyl]-2-fluorobenzo[ $\left.b\right]$ thieno[2,3-d]thiophene $\mathbf{3 g}$

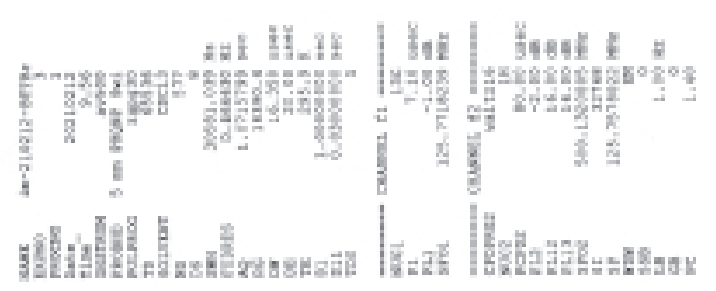<smiles>Cc1oc2c(oc3ccccc32)c1Oc1ccccc1Cl</smiles>

8)
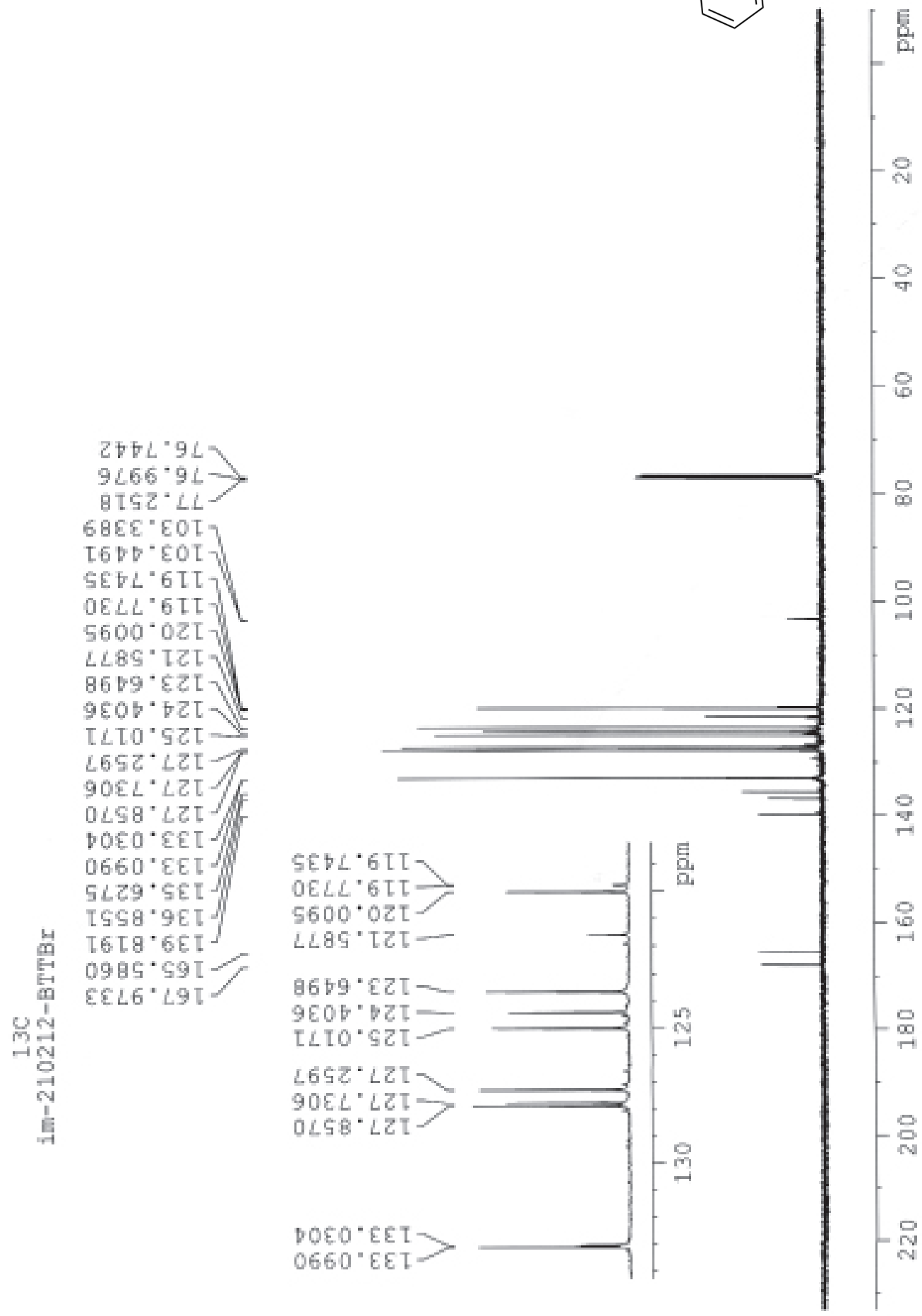
${ }^{19}$ F NMR Spectrum of 3-[(2-Bromophenyl)sulfanyl]-2-fluorobenzo[b]thieno[2,3-d] thiophene 3g
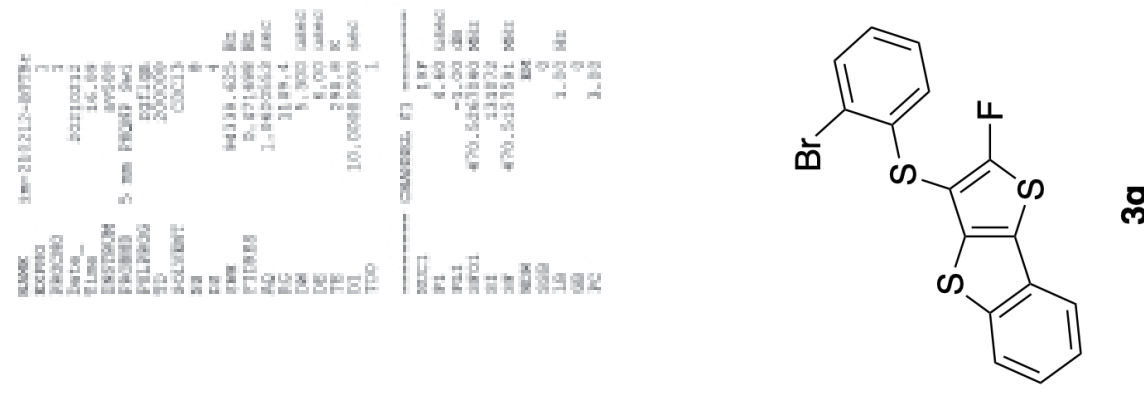

$0000^{\circ} 0-$

$68 L S^{\circ} 0 S_{-}$

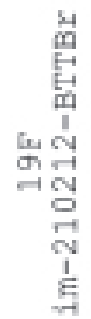

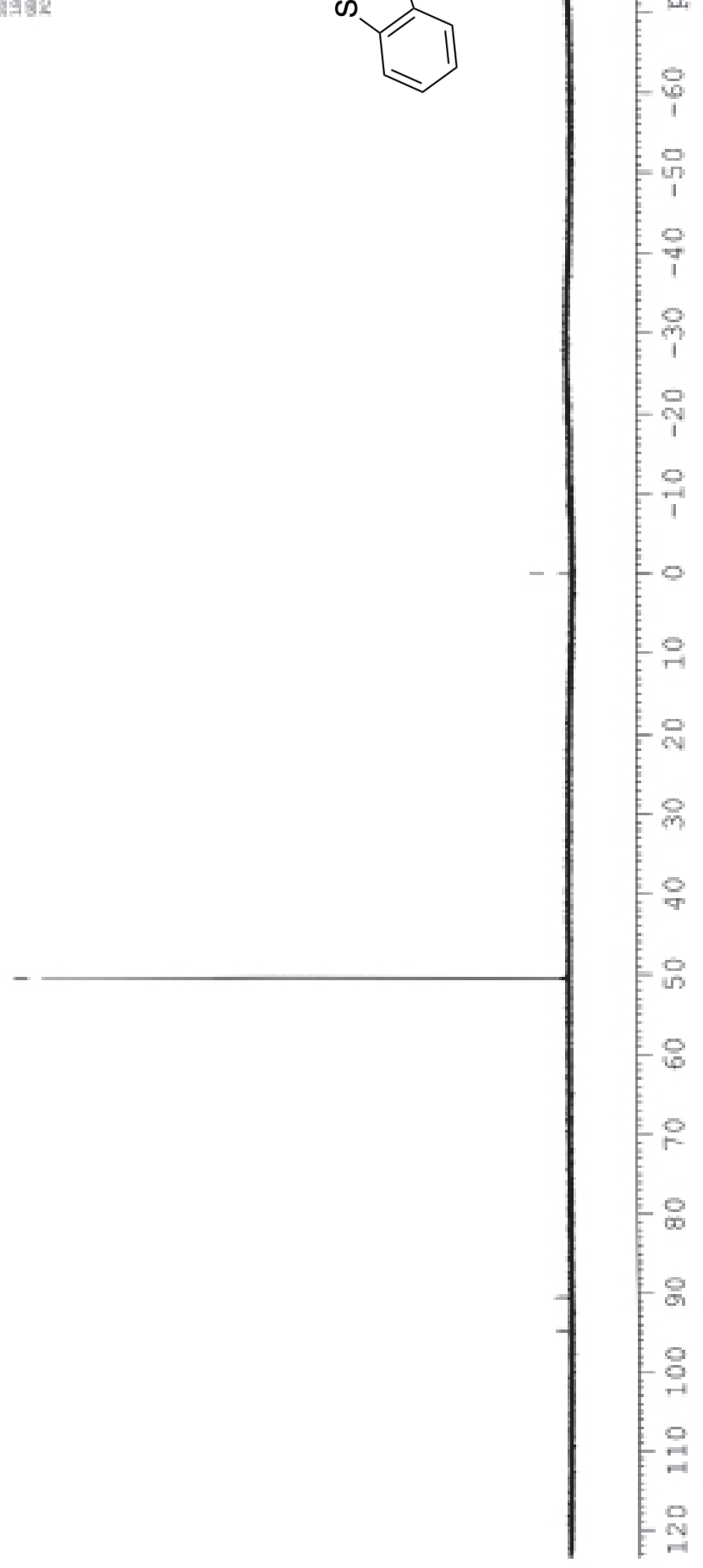


${ }^{1}$ H NMR Spectrum of 2-Fluoro-3-(phenylsulfanyl)thieno[2,3-b]thiophene $\mathbf{3 h}$
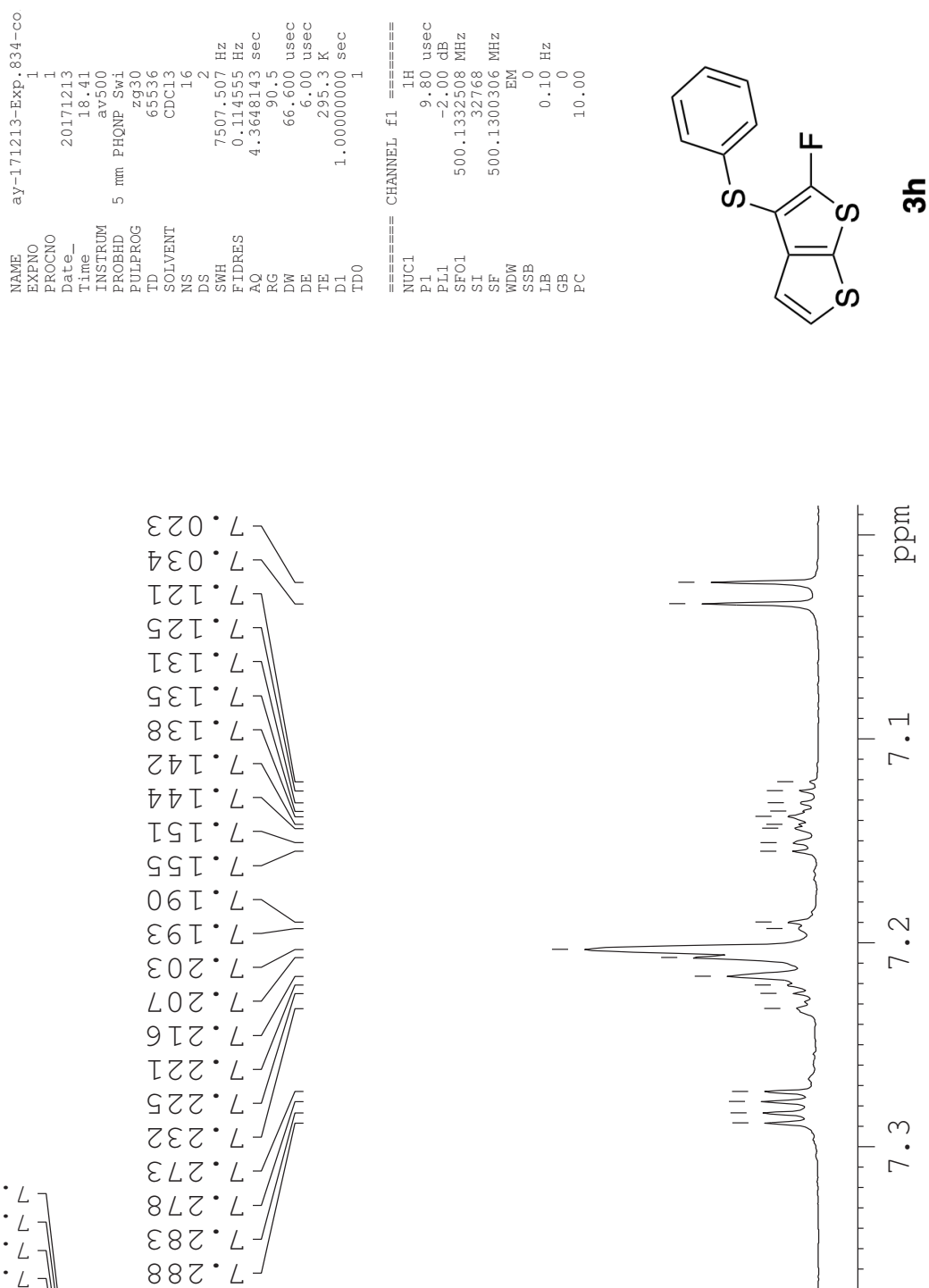

โSZT. $88 \mathrm{Z}^{\circ} \mathrm{L}^{\circ}$

$\varepsilon[\varepsilon \tau \cdot L]$

$\nabla \subseteq \varepsilon[\cdot L-$

$08 \varepsilon[\cdot L]$

$6[\nabla[\cdot L]$

$8 \varepsilon \varpi \tau \cdot L]$

$60 S T \cdot L$

$868 I^{\circ} \cdot L$

$0 \varepsilon 6 \tau \cdot L$

乙ह०२ $L$

$\mathrm{ZLOZ} \cdot \mathrm{LJ}^{\prime}$

79[Z・LJ

$\angle O Z Z \cdot L J$

$8 \pi z Z \cdot L$

乙てとて・L

$6 Z L Z \cdot L$

$8 L L Z \cdot L-$



, $\varepsilon 882^{\circ} L$

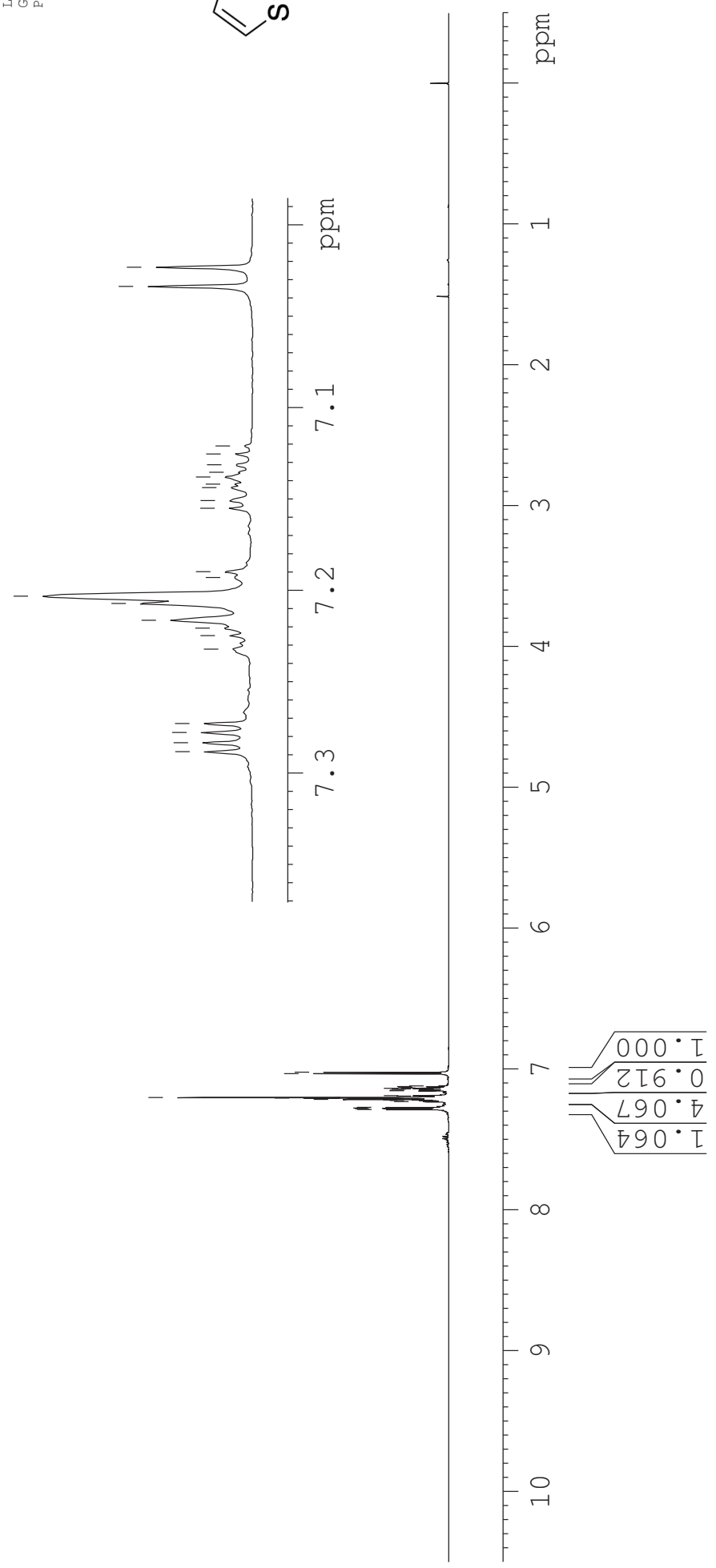


${ }^{13} \mathrm{C}$ NMR Spectrum of 2-Fluoro-3-(phenylsulfanyl)thieno[2,3-b]thiophene $\mathbf{3 h}$

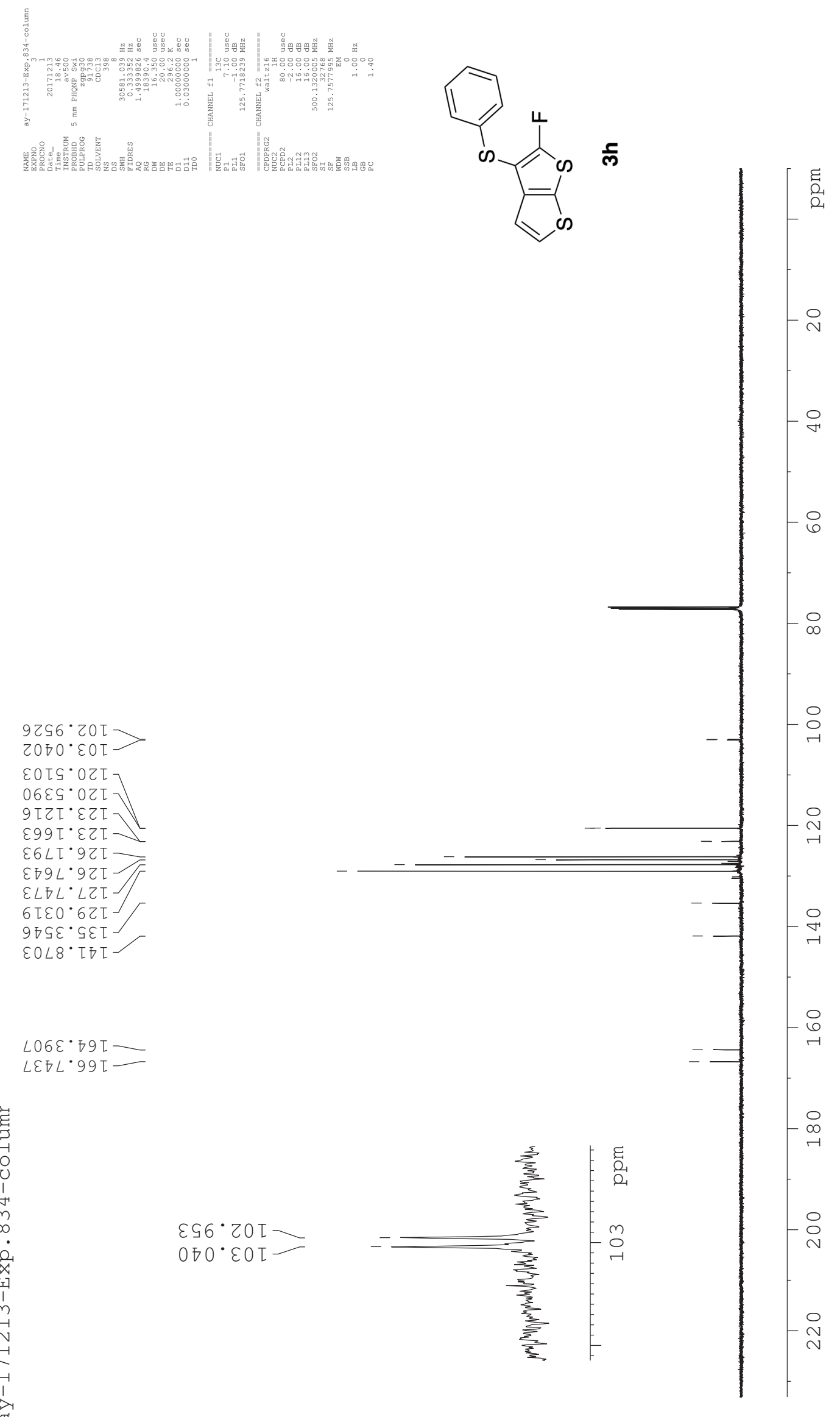


${ }^{19}$ F NMR Spectrum of 2-Fluoro-3-(phenylsulfanyl)thieno[2,3-b]thiophene $\mathbf{3 h}$

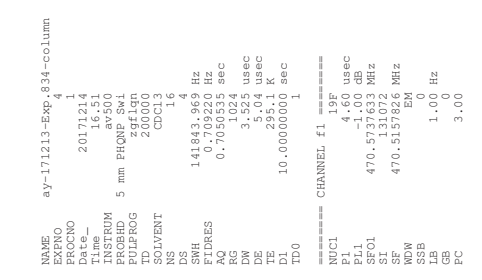

$\varepsilon[2 S \cdot 8 \pi>$

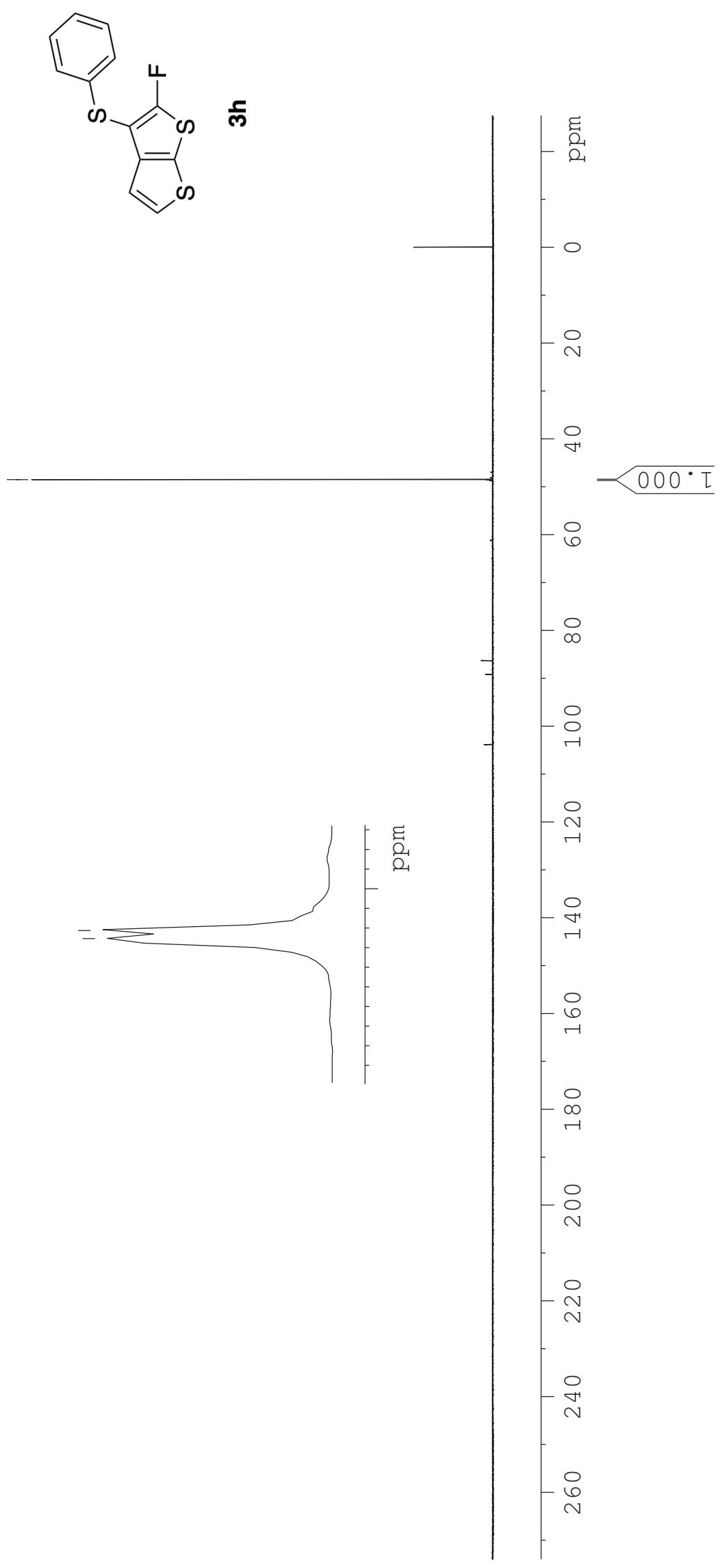

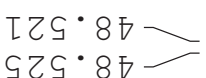


${ }^{1}$ H NMR Spectrum of 2-Fluoro-5-methyl-3-(phenylsulfanyl)thieno[2,3-b]thiophene 3i

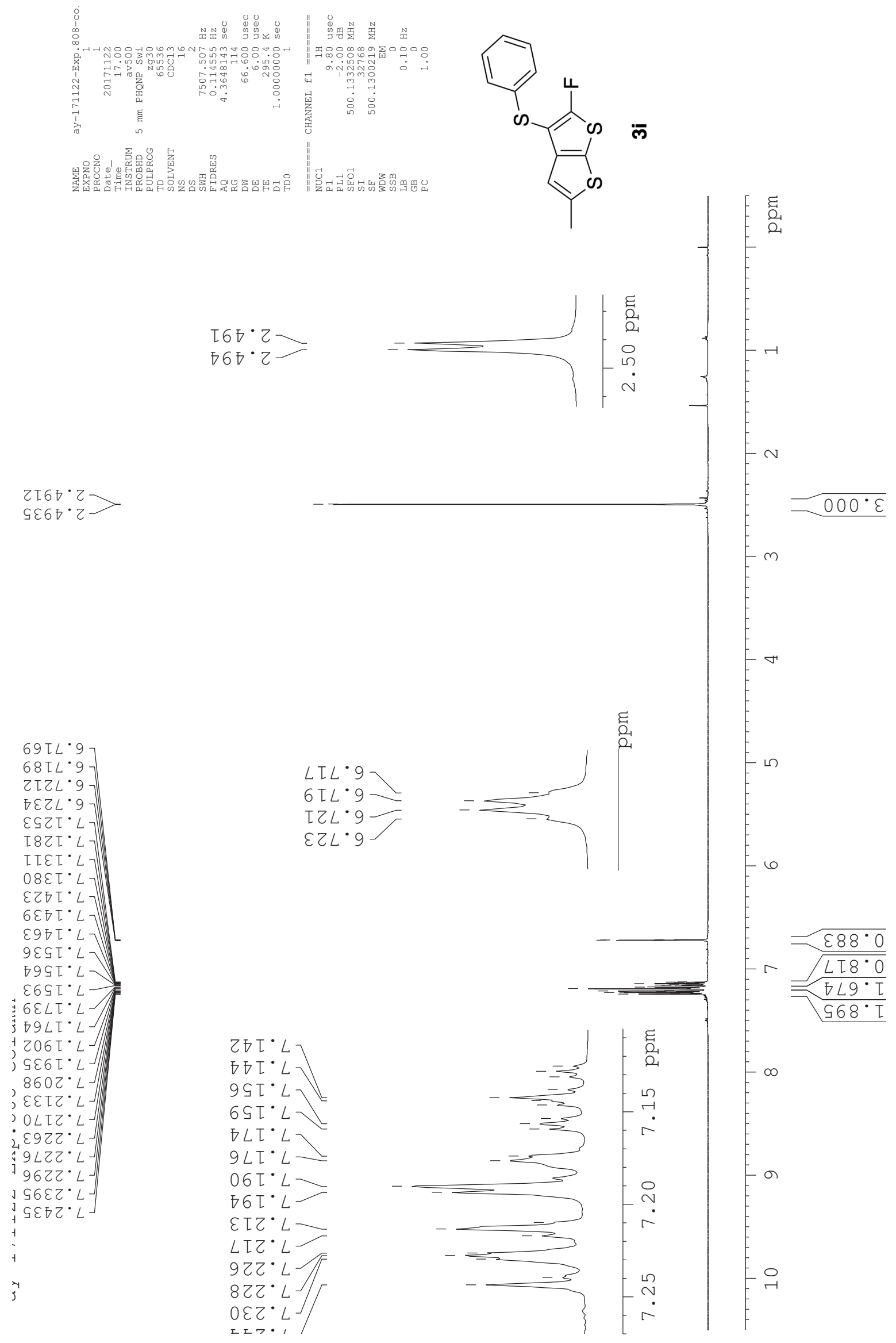


${ }^{13} \mathrm{C}$ NMR Spectrum of 2-Fluoro-5-methyl-3-(phenylsulfanyl)thieno[2,3-b]thiophene 3i
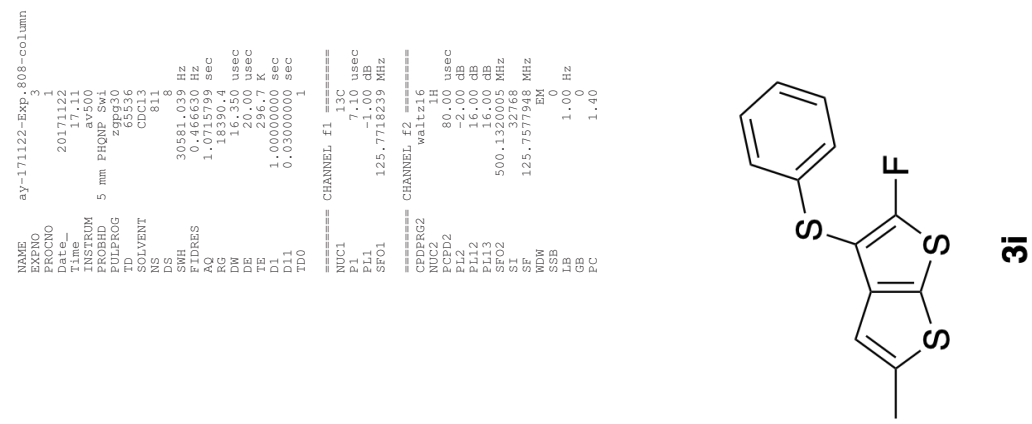

$6 \varepsilon \nabla \sigma^{\circ} \varsigma \tau$

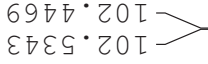

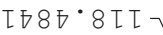

$060 S^{\circ} 8 \mathrm{II}$

OZ๐॰.0ZL

988 ${ }^{\circ} 0 Z \mathrm{~T}$

67t0.9ZT

OLLF' $\angle Z T$

$82200^{\circ} 62 \mathrm{I}$

$2679^{\circ} \mathrm{SEL}$

LZ99. ५Eโ

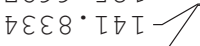

๑0七 $6^{\circ}$ 切

ง9โE. เ9I-

7299.99T -

$\vec{y}$
5
-1
0
0
0
1
0
0
0
0
0
0
$x$
1
1
0
$N$
7
7
7
7
7
1
2
2

$\angle D t \cdot 20 I-$

DEG'ZOI

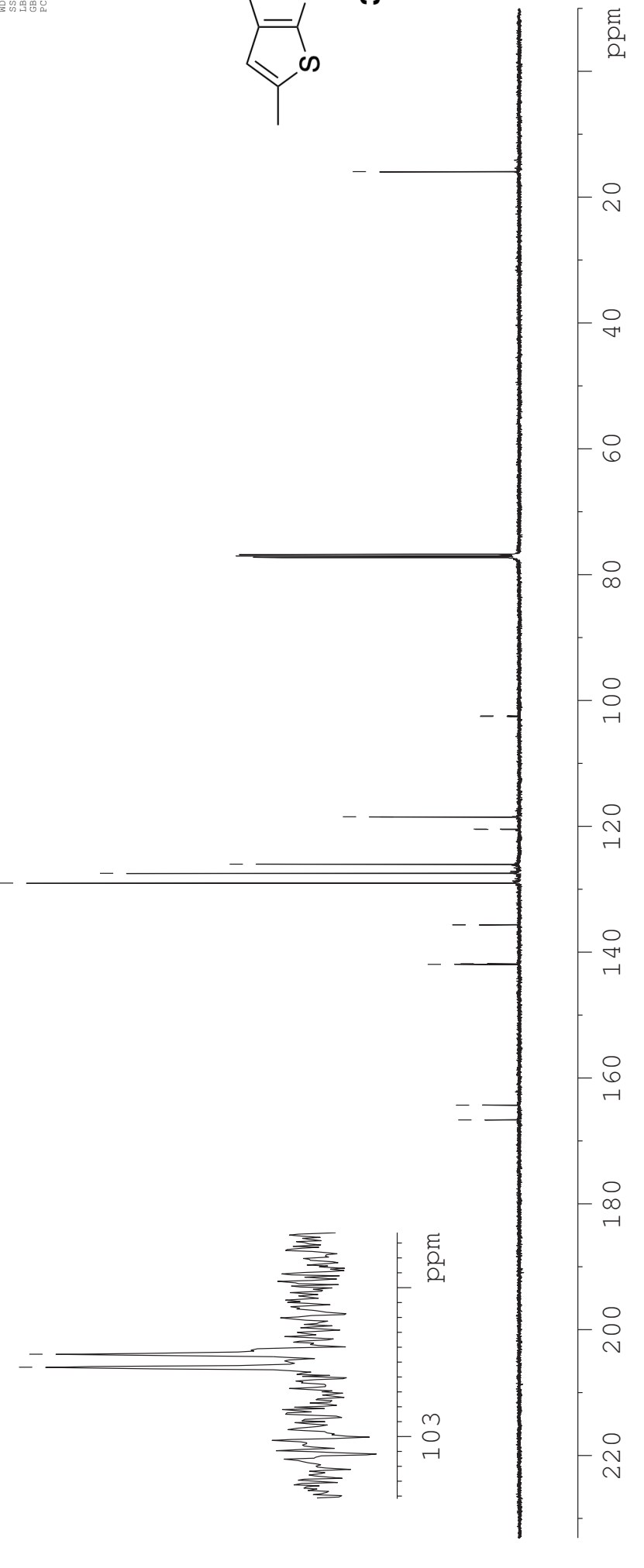


${ }^{19}$ F NMR Spectrum of 2-Fluoro-5-methyl-3-(phenylsulfanyl)thieno[2,3-b]thiophene 3i
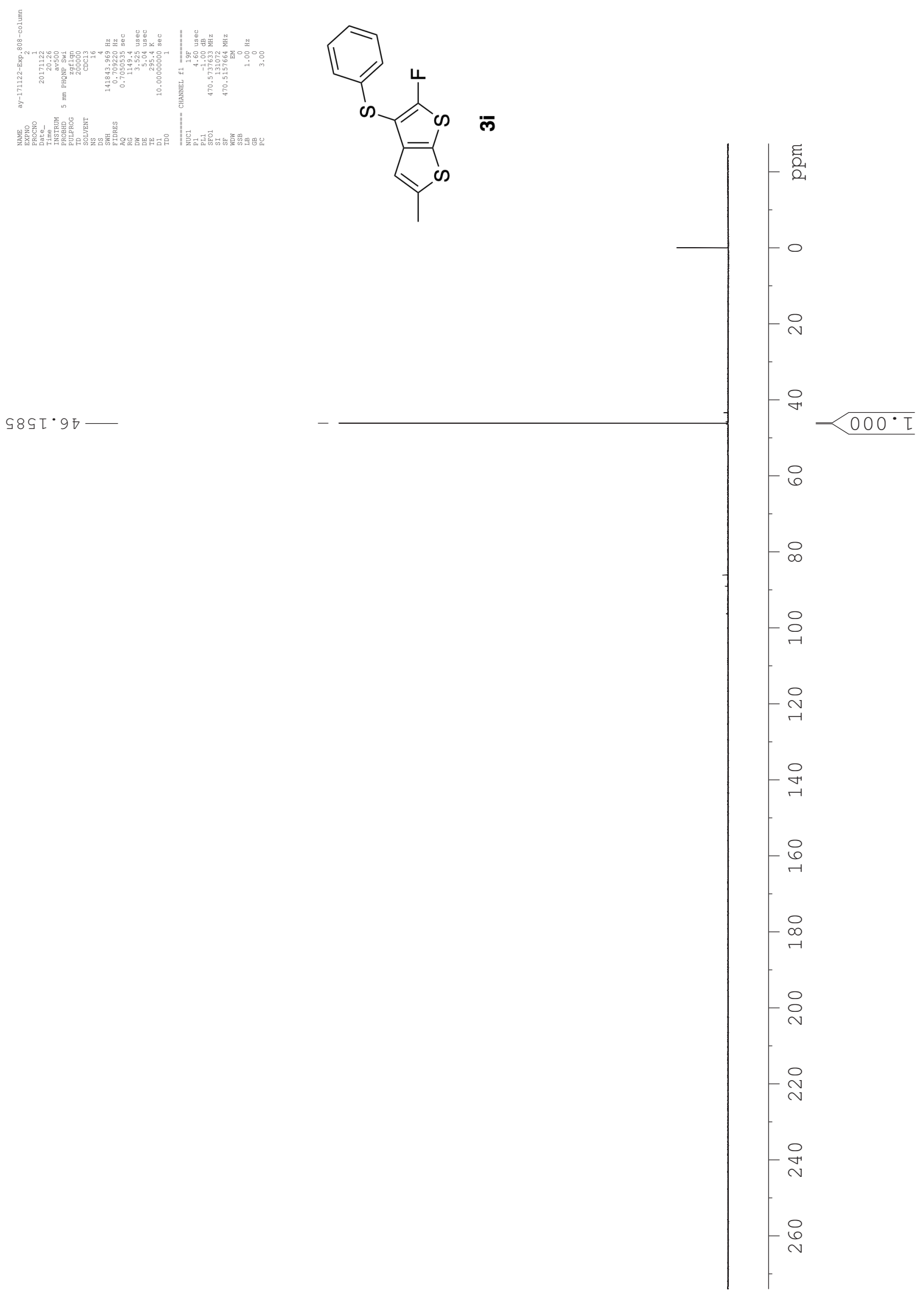
${ }^{1} \mathrm{H}$ NMR Spectrum of 2-Fluoro-5-phenyl-3-(phenylsulfanyl)thieno[2,3-b]thiophene $\mathbf{3 j}$
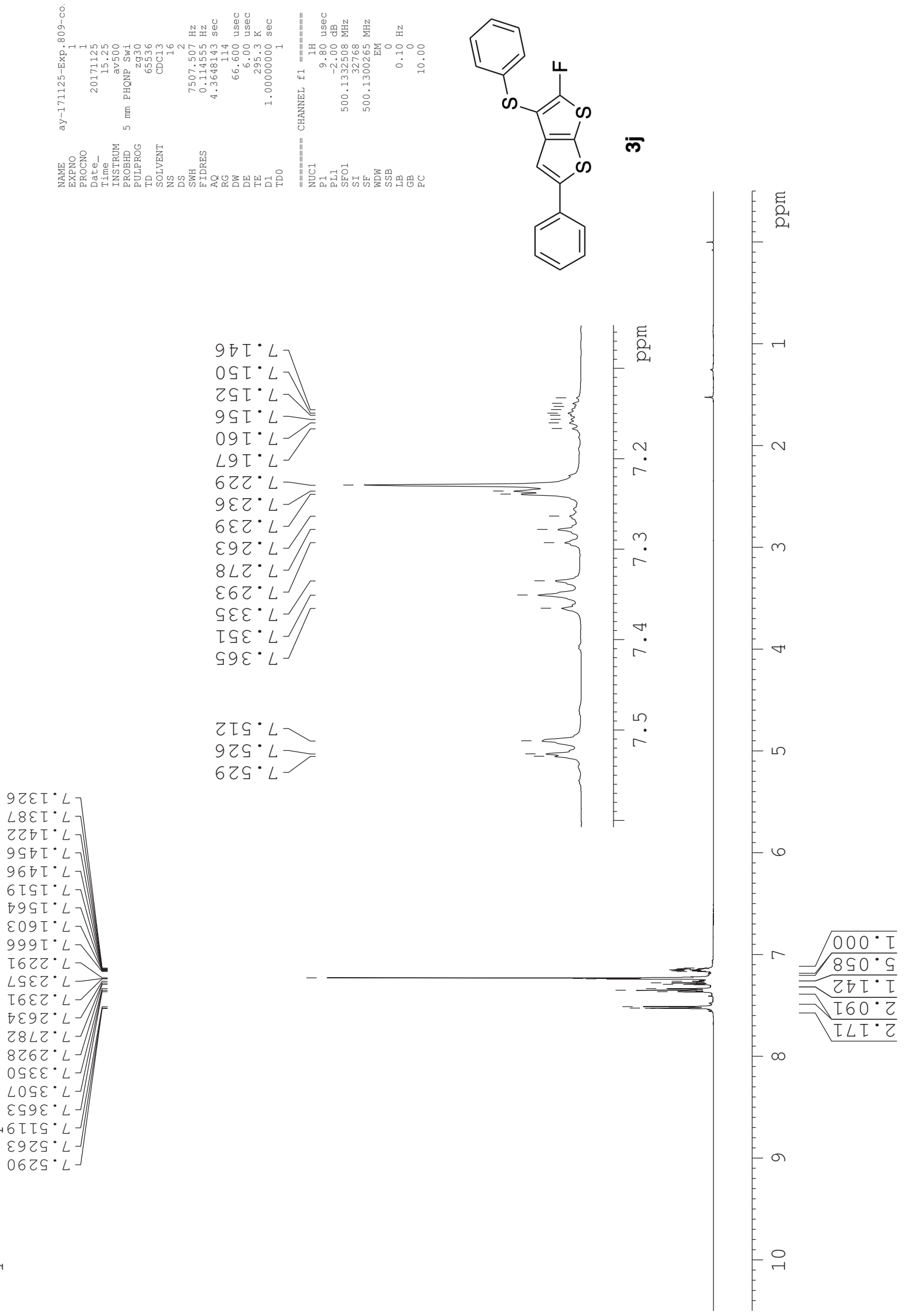
${ }^{13} \mathrm{C}$ NMR Spectrum of 2-Fluoro-5-phenyl-3-(phenylsulfanyl)thieno[2,3-b]thiophene $\mathbf{3 j}$
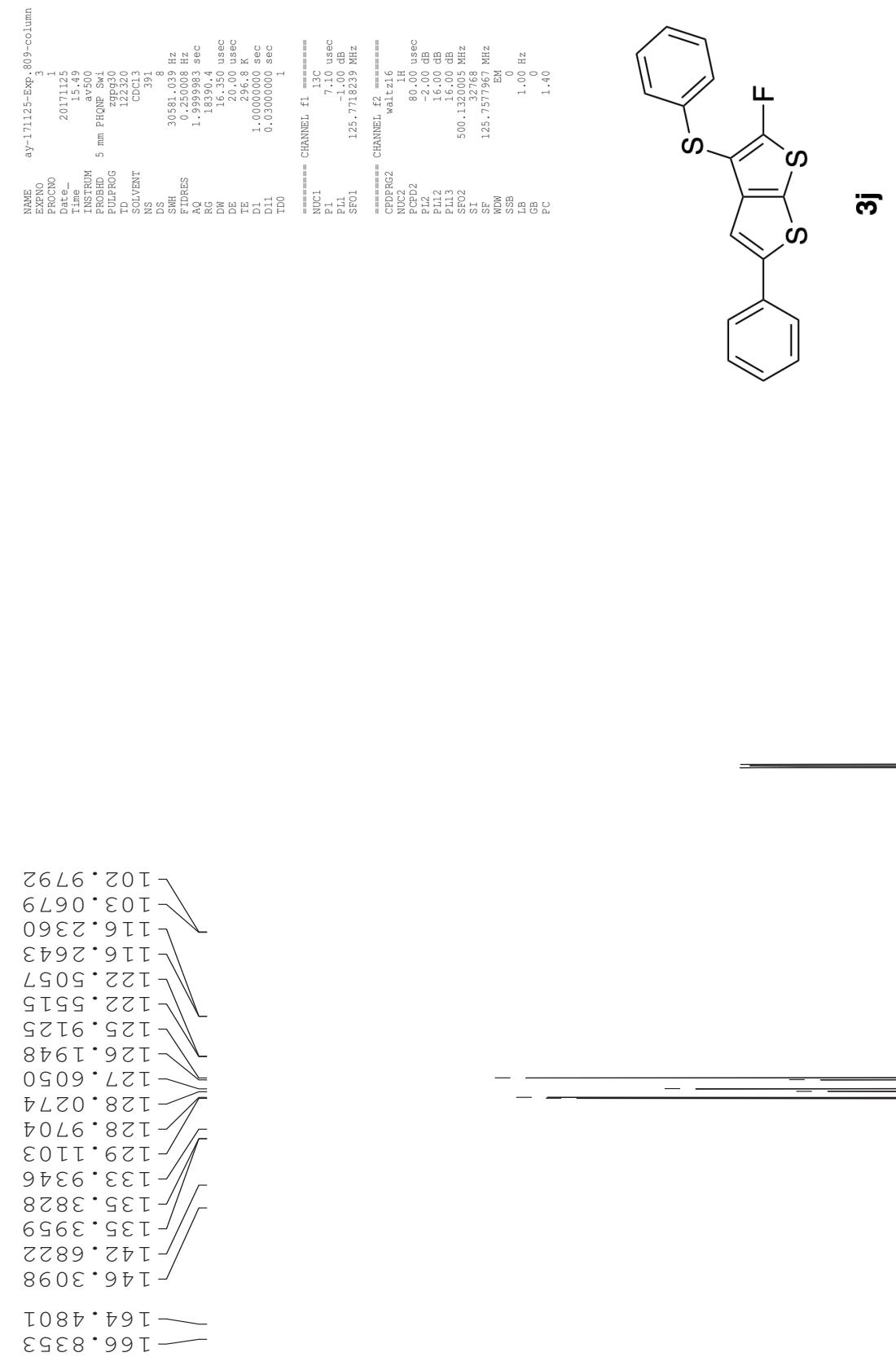

$6 \angle 6^{\circ} 20 T$ $890^{\circ} \varepsilon O T$

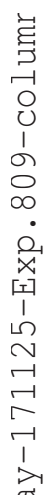

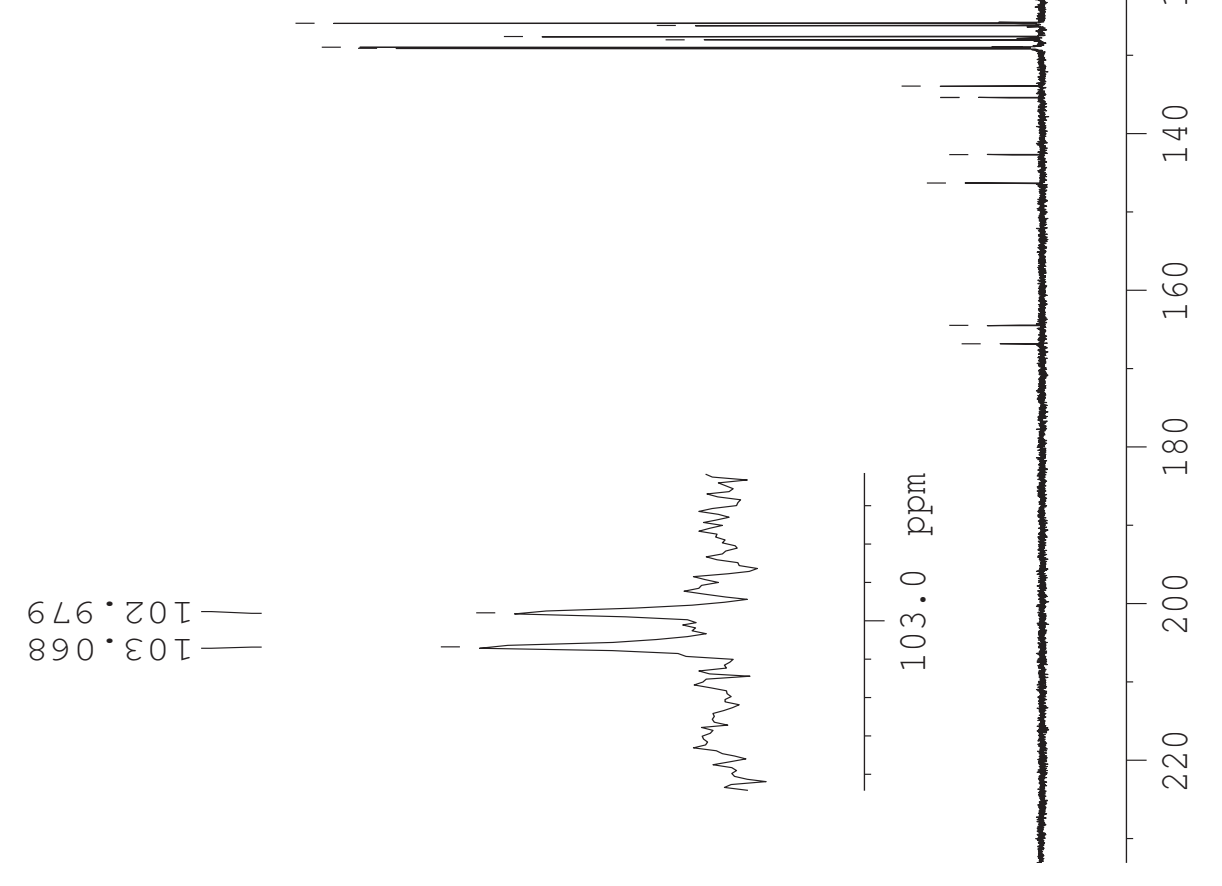


${ }^{19}$ F NMR Spectrum of 2-Fluoro-5-phenyl-3-(phenylsulfanyl)thieno[2,3-b]thiophene 3j
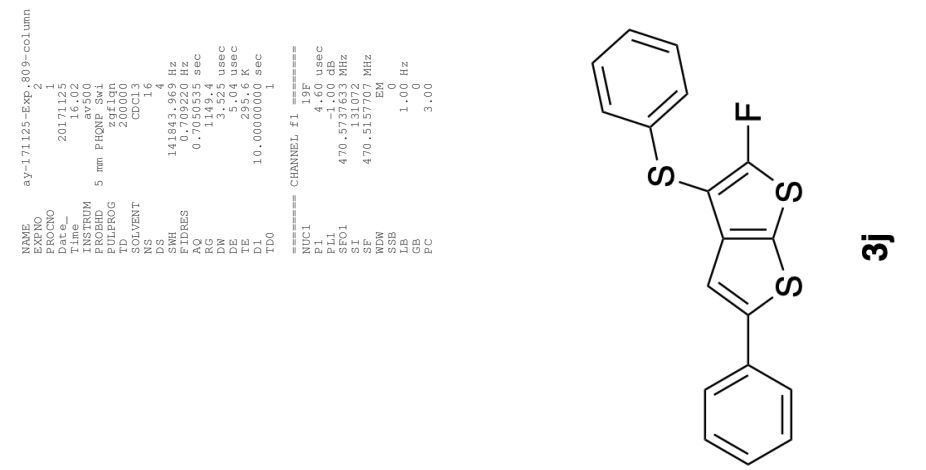

D089 $\angle 7$

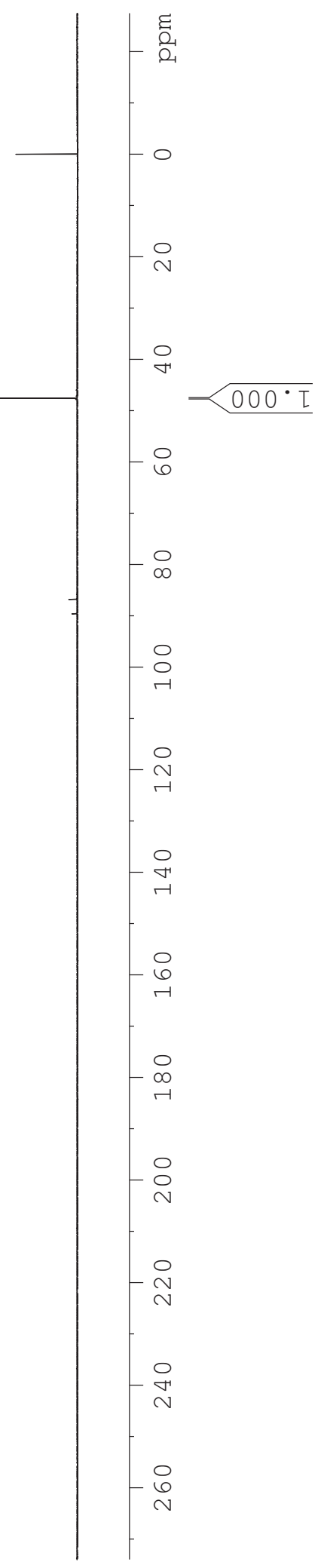


${ }^{1} \mathrm{H}$ NMR Spectrum of 5-Bromo-2-fluoro-3-(phenylsulfanyl)thieno[2,3-b]thiophene 3k
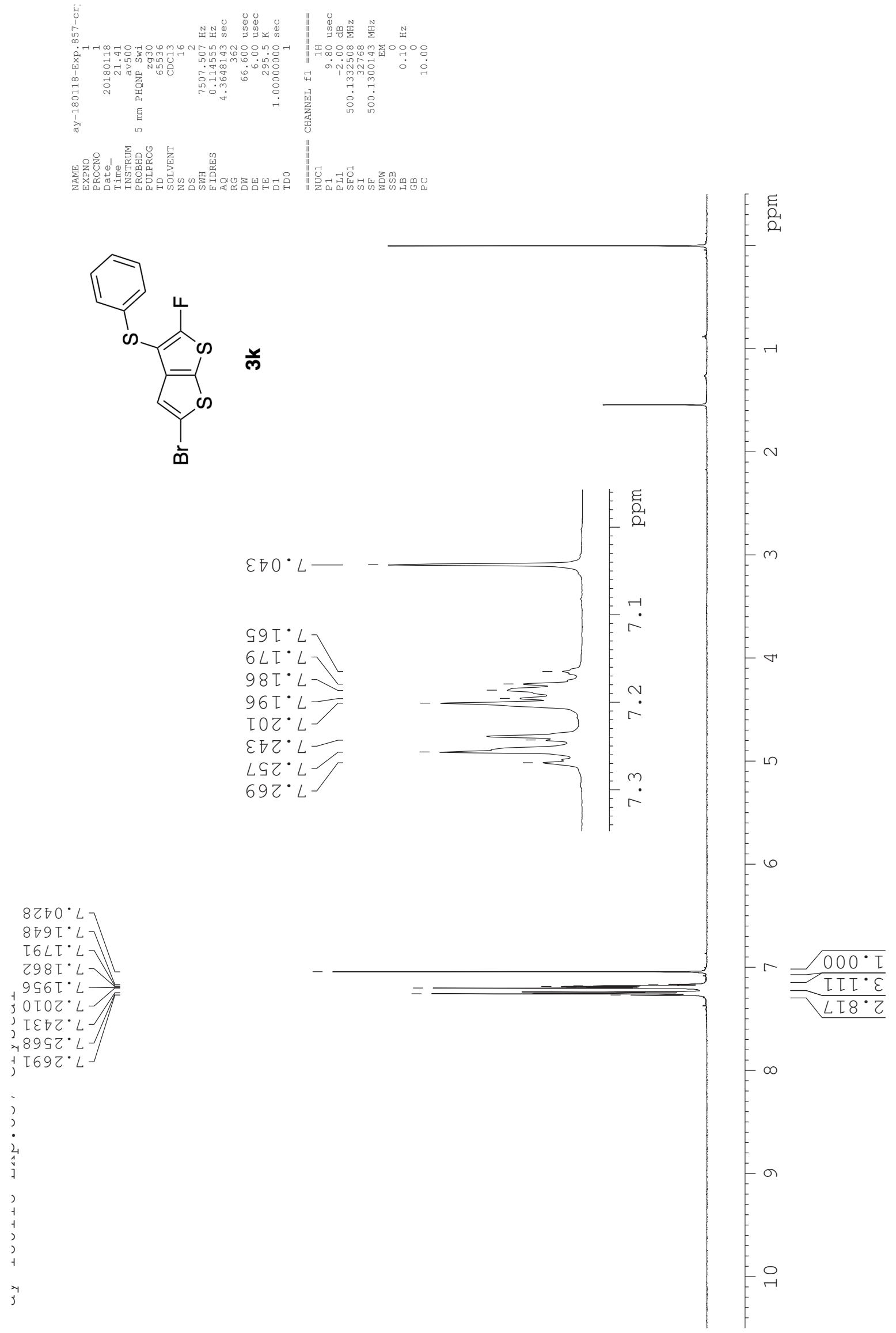
${ }^{13} \mathrm{C}$ NMR Spectrum of 5-Bromo-2-fluoro-3-(phenylsulfanyl)thieno[2,3-b]thiophene 3k

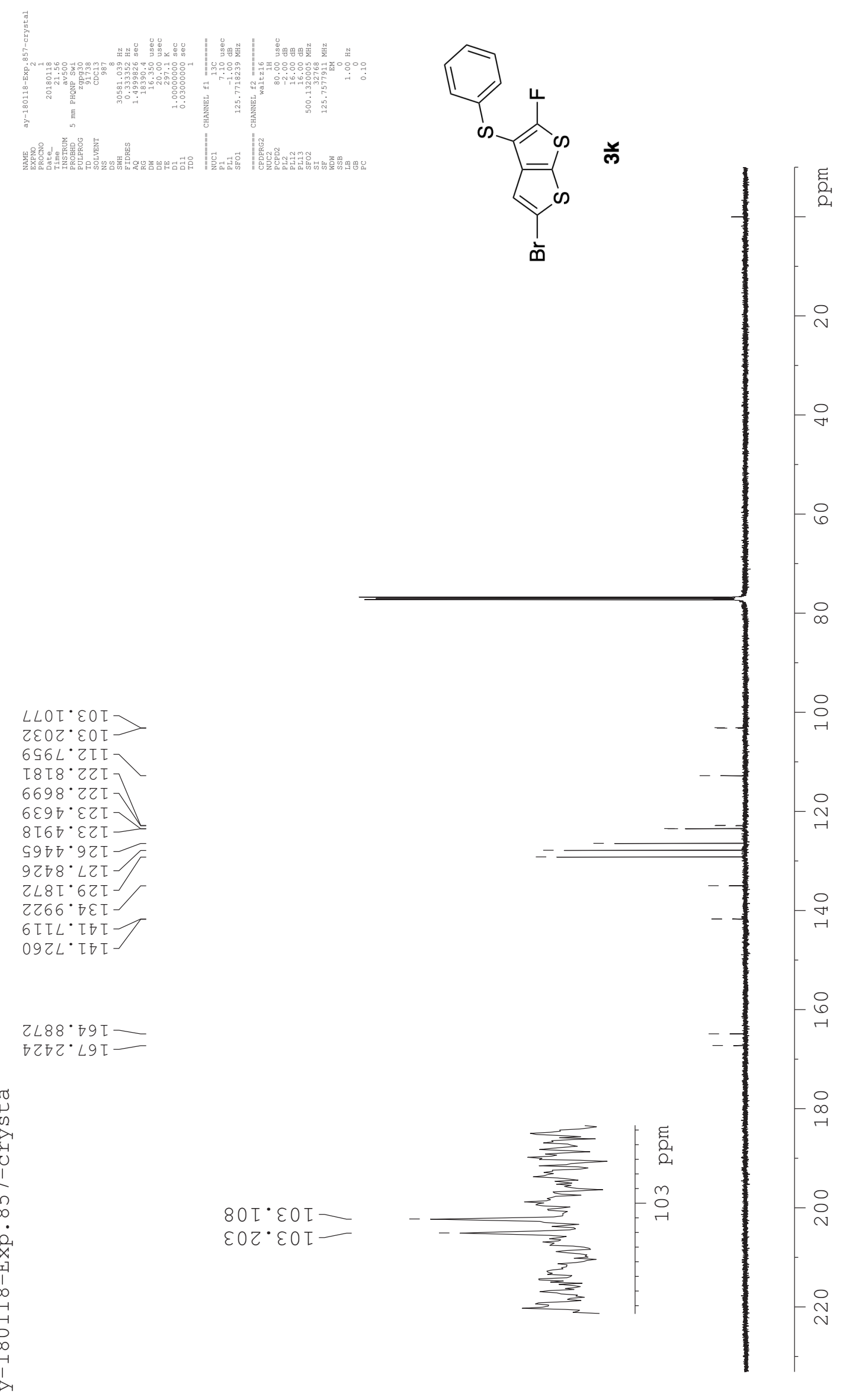


${ }^{19}$ F NMR Spectrum of 5-Bromo-2-fluoro-3-(phenylsulfanyl)thieno[2,3-b]thiophene $\mathbf{3 k}$

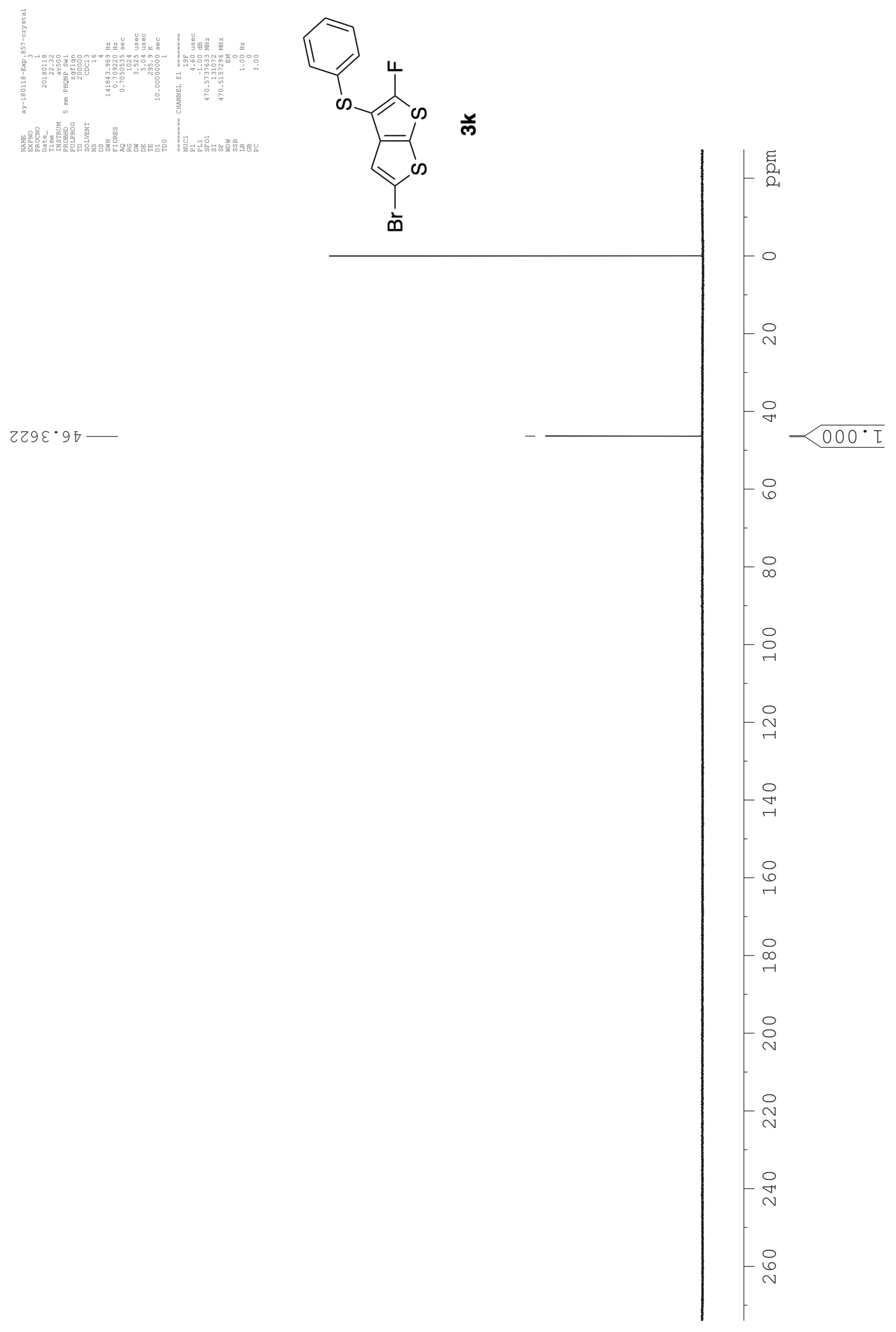


${ }^{1} \mathrm{H}$ NMR Spectrum of 2-Fluoro-3-[(4-methoxyphenyl)sulfanyl] thieno[2,3-b]thiophene $3 \mathbf{l}$
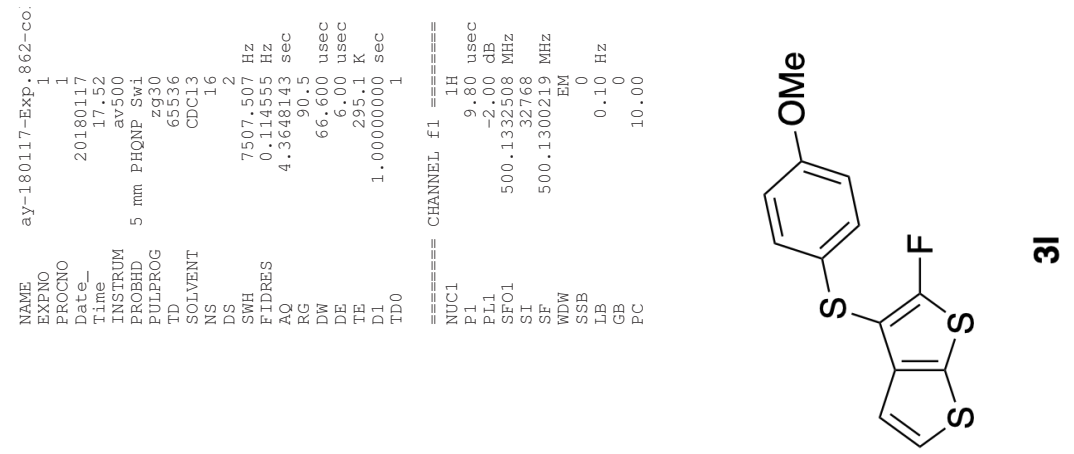

$697 L \cdot \varepsilon-$

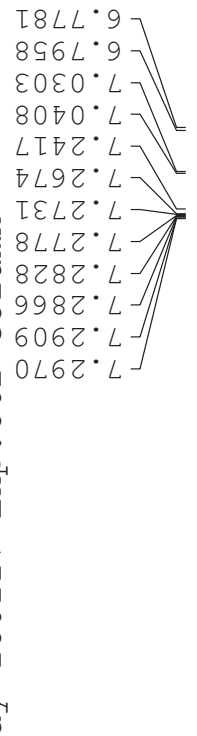

$8 L L \cdot 9=$
$96 L \cdot 9=$

OEO $0^{*} L$

โ两 $0^{\circ} \mathrm{L}$

乙ゅ乙・

$\angle 9 Z^{\cdot} L$

$\varepsilon\left\llcorner Z^{\cdot}\right.$

$8 L Z \cdot L$

$\varepsilon 8 \tau^{\circ}$

$\angle 8 Z^{\circ}$

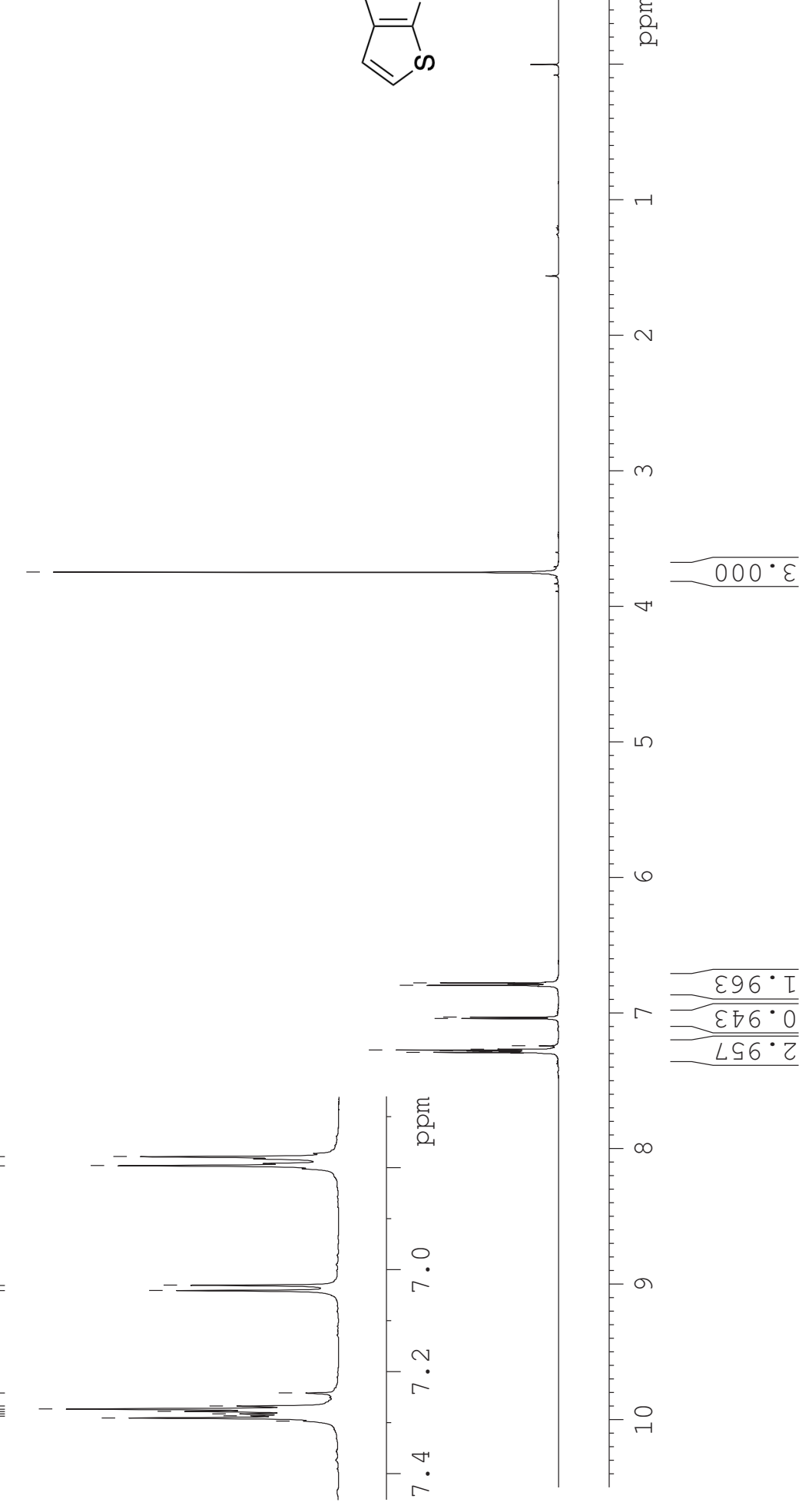


${ }^{13}$ C NMR Spectrum of 2-Fluoro-3-[(4-methoxyphenyl)sulfanyl]thieno[2,3-b] thiophene 3I
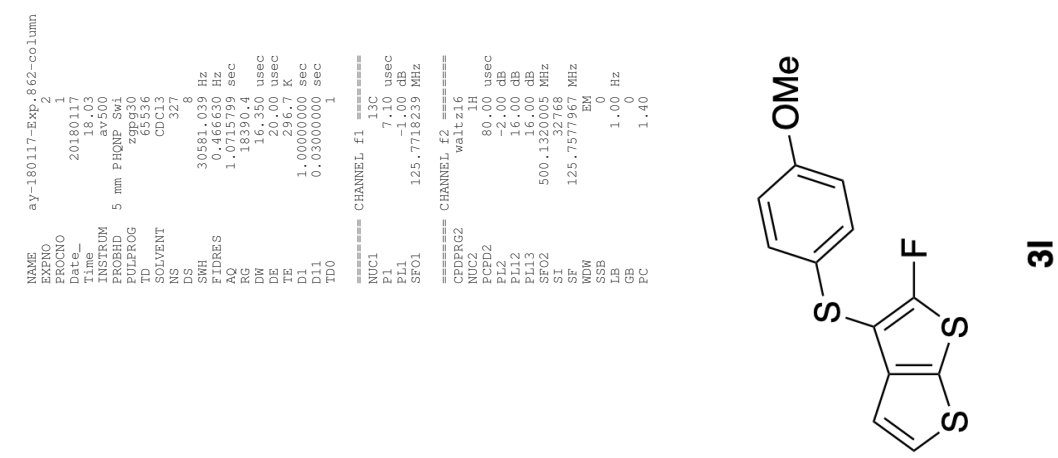

$098 Z$ `S

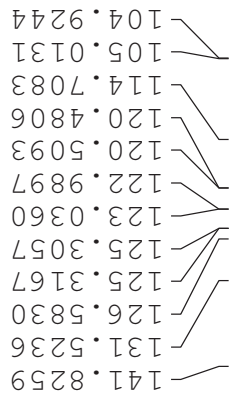

ZLS6.8ST

Ð

$0989^{\circ} 99 \mathrm{I}$

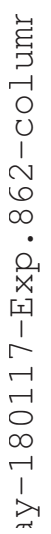

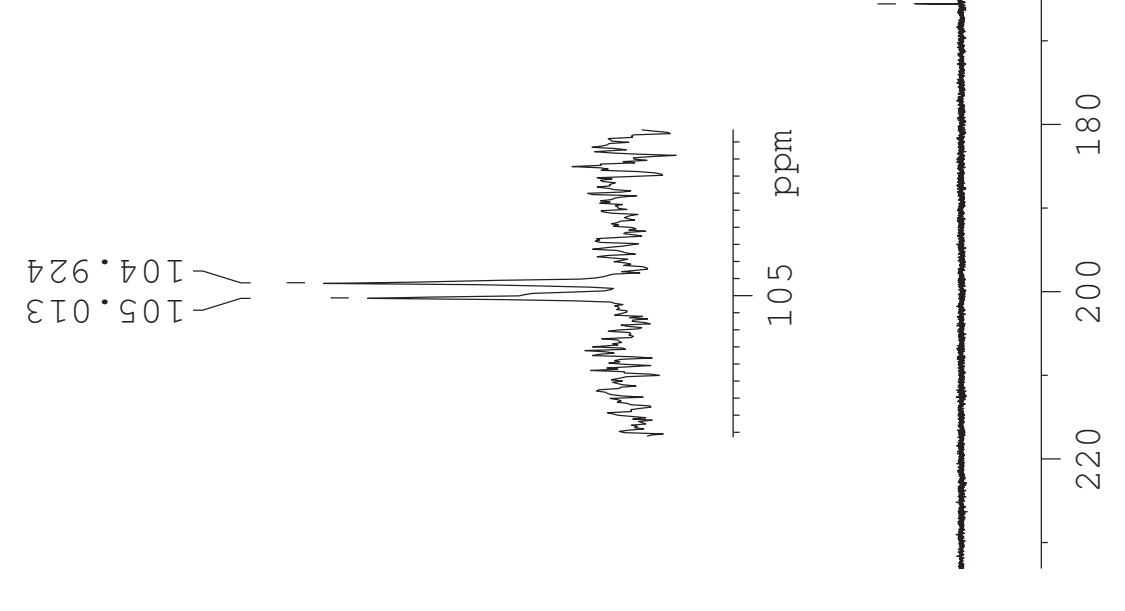


${ }^{19}$ F NMR Spectrum of 2-Fluoro-3-[(4-methoxyphenyl)sulfanyl]thieno[2,3-b]thiophene 31
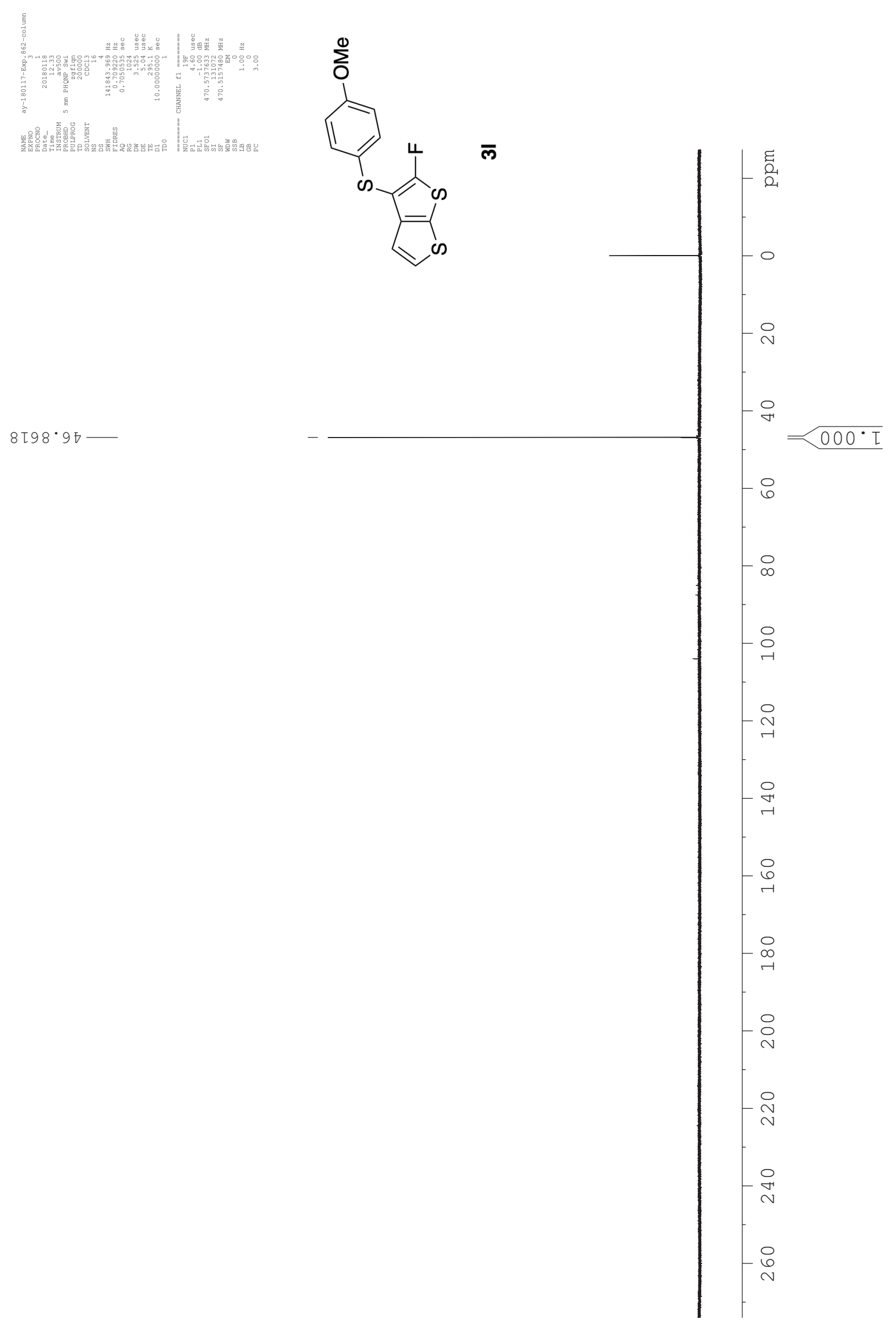

$8 โ 98 \cdot 97-$ 
${ }^{1}$ H NMR Spectrum of 2-Fluoro-3-[(4-chlorophenyl)sulfanyl]thieno[2,3-b]thiophene 3m

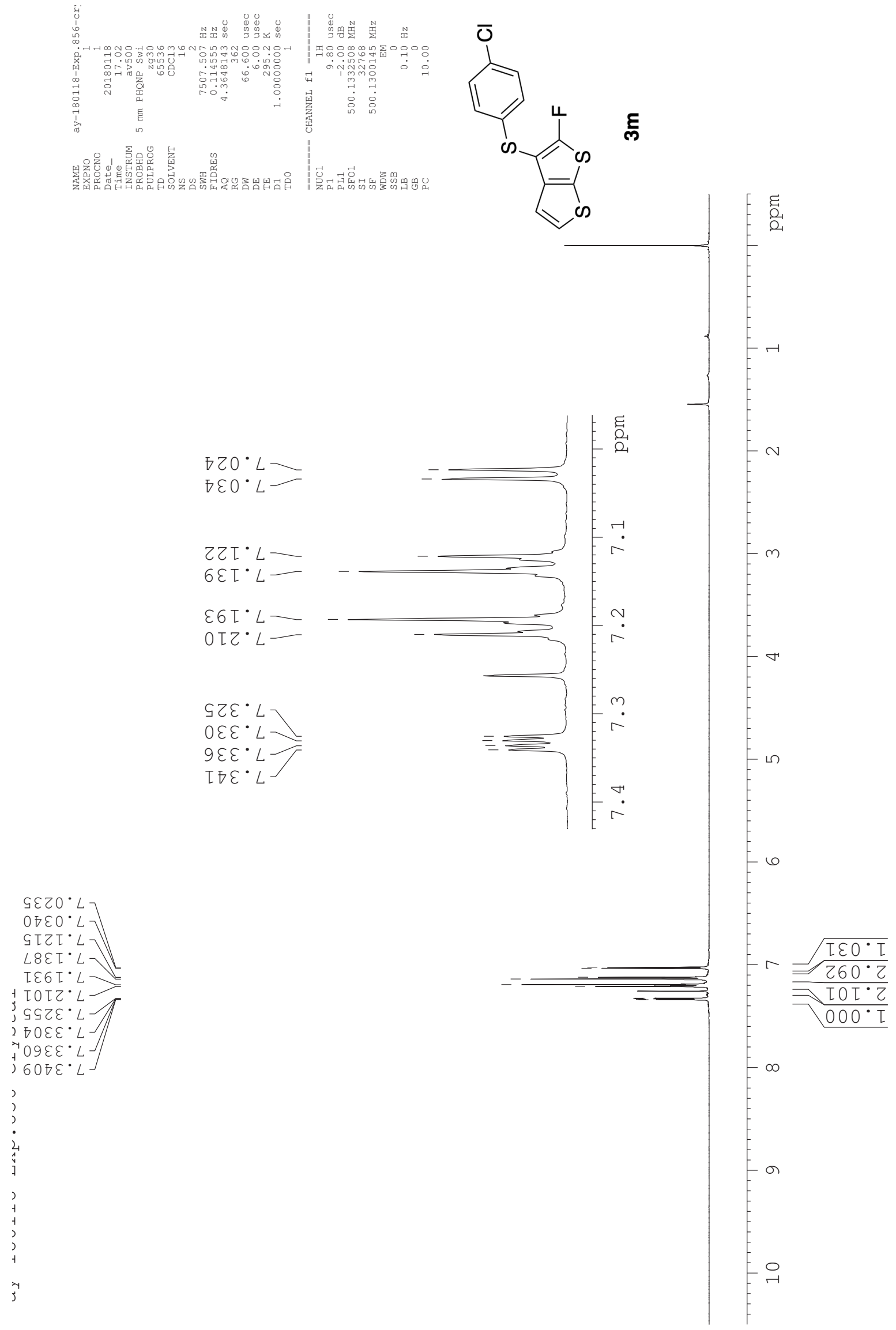


${ }^{13} \mathrm{C}$ NMR Spectrum of 2-Fluoro-3-[(4-chlorophenyl)sulfanyl]thieno[2,3-b]thiophene $\mathbf{3 m}$

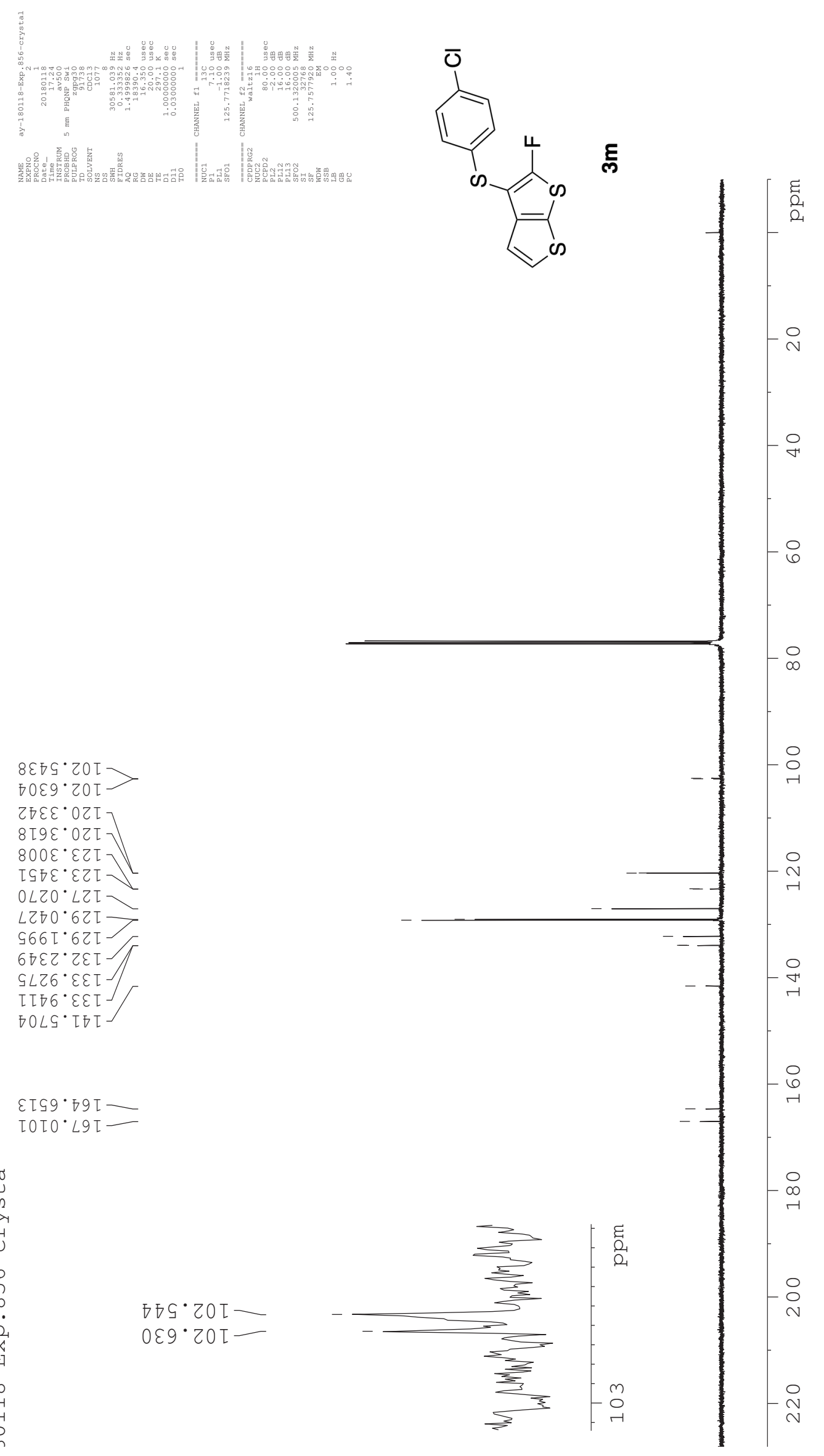


${ }^{19}$ F NMR Spectrum of 2-Fluoro-3-[(4-chlorophenyl)sulfanyl]thieno[2,3-b]thiophene $\mathbf{3 m}$

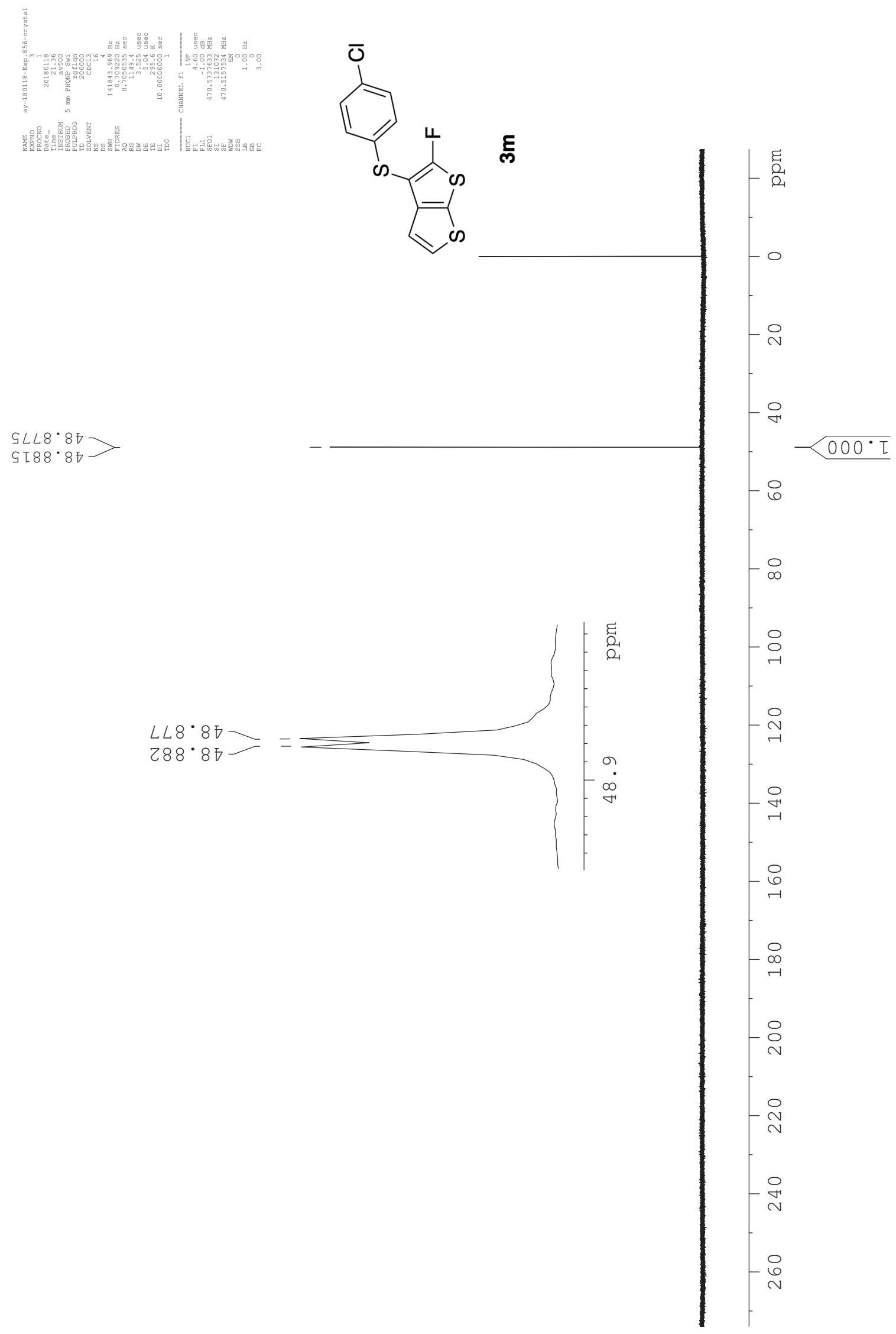


${ }^{1} \mathrm{H}$ NMR Spectrum of 2-Fluoro-3-(phenylsulfanyl)benzo[b]thieno[3,2- $\left.d\right]$ thiophene 3n<smiles>Cc1oc2oc3ccccc3c2c1Oc1ccccc1</smiles>

ฉ్

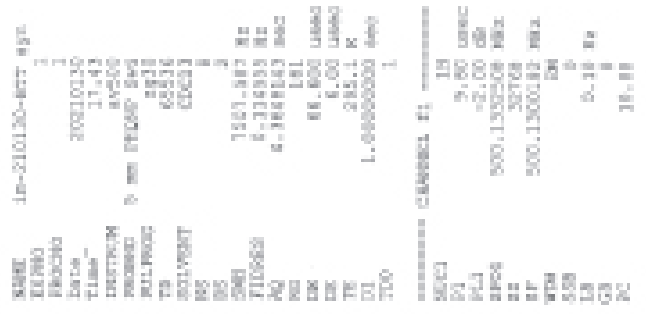

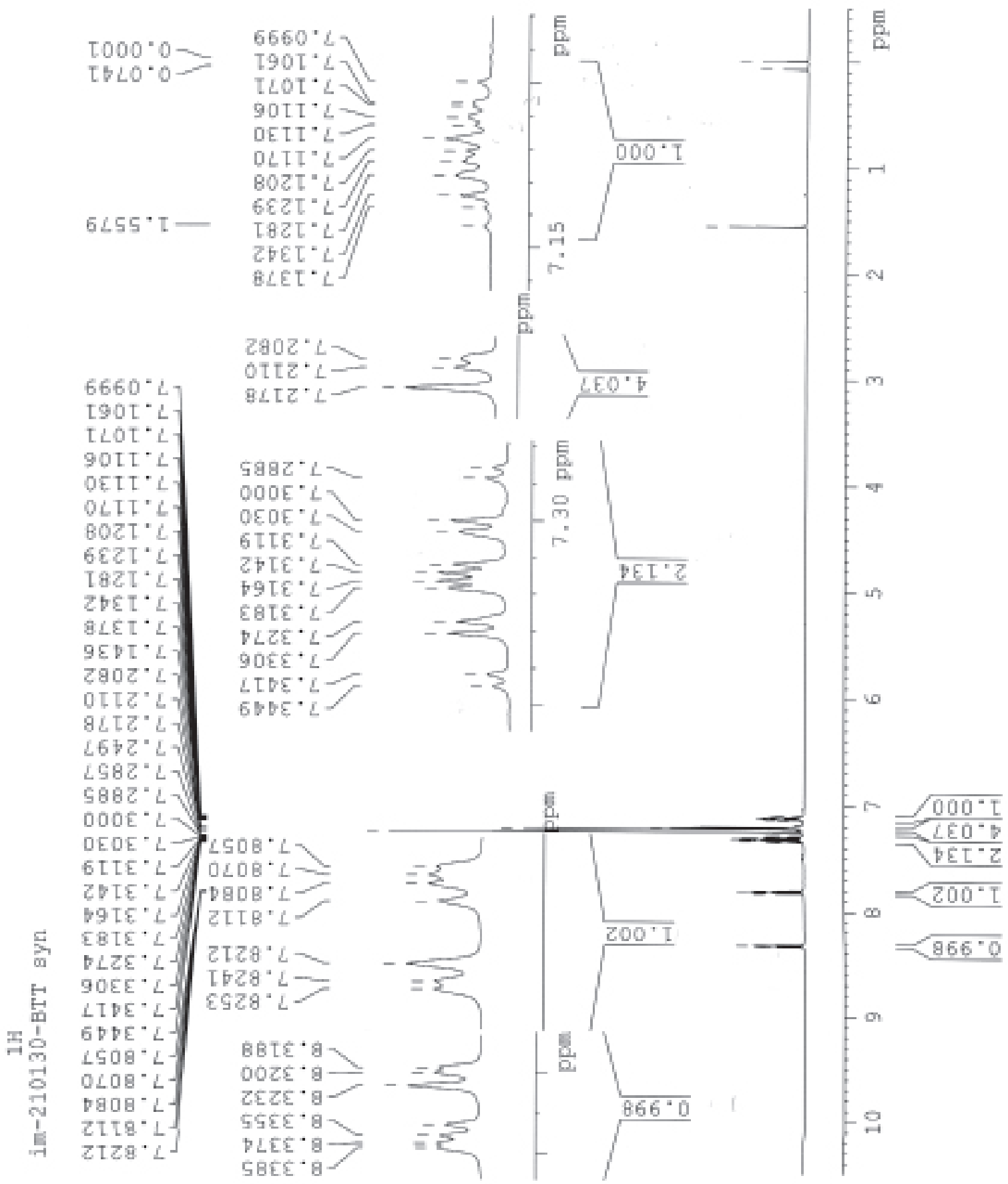


${ }^{13} \mathrm{C}$ NMR Spectrum of 2-Fluoro-3-(phenylsulfanyl)benzo[b]thieno[3,2- $\left.d\right]$ thiophene 3n

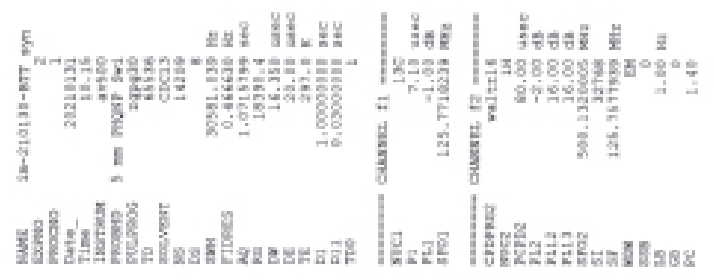<smiles>Cc1oc2oc3ccccc3c2c1Oc1ccccc1</smiles>

ณิ

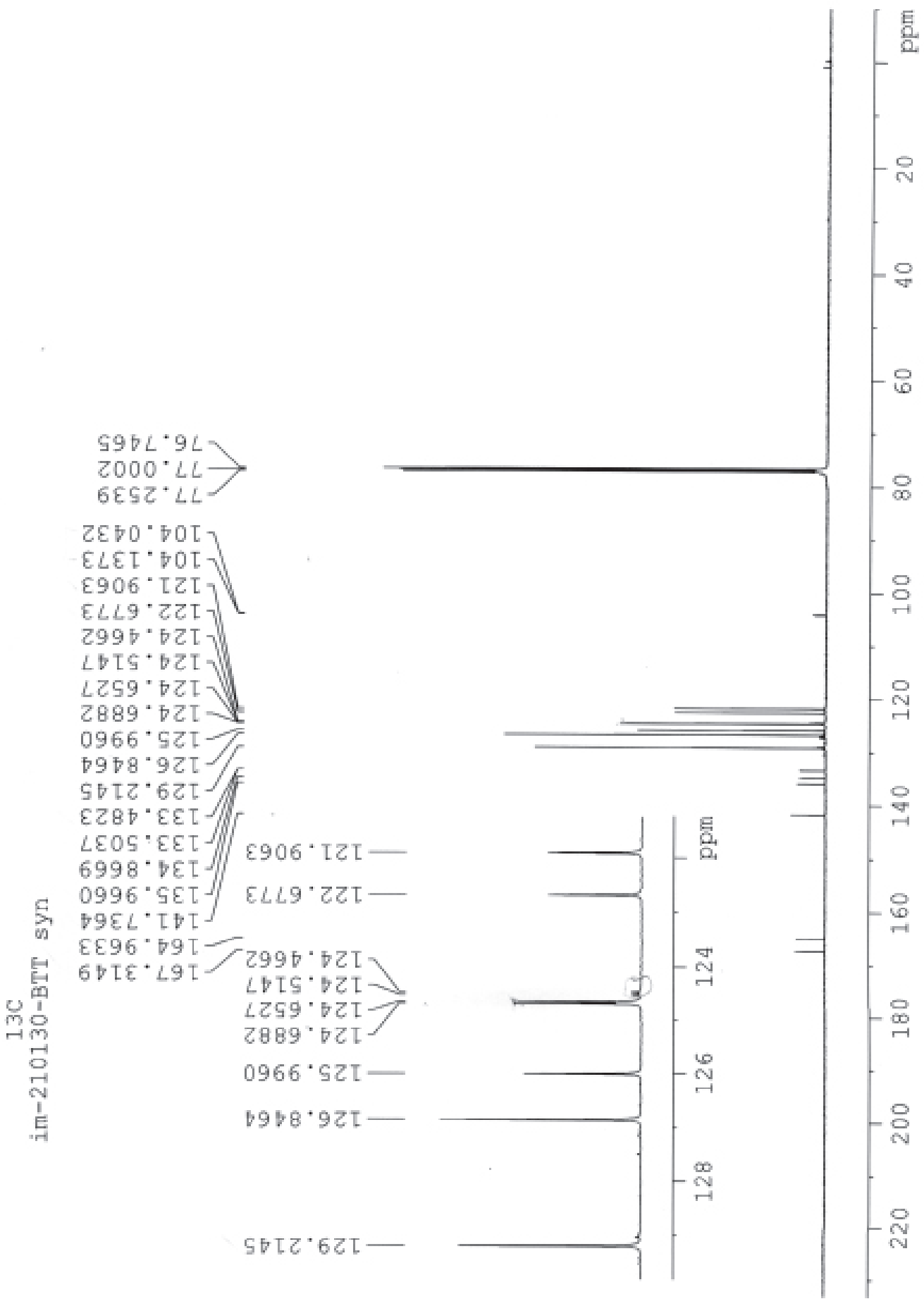


${ }^{19}$ F NMR Spectrum of 2-Fluoro-3-(phenylsulfanyl)benzo[ $\left.b\right]$ thieno[3,2- $\left.d\right]$ thiophene 3n
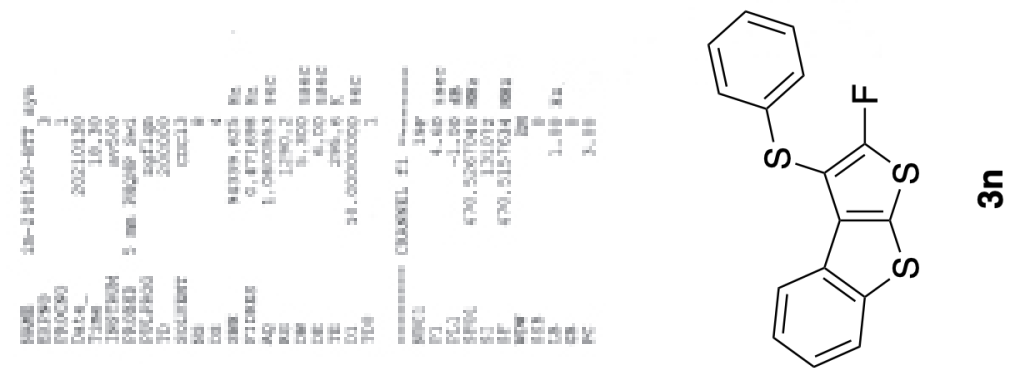

$5000^{\circ} 0=$

$8860^{\circ} 87-$

is

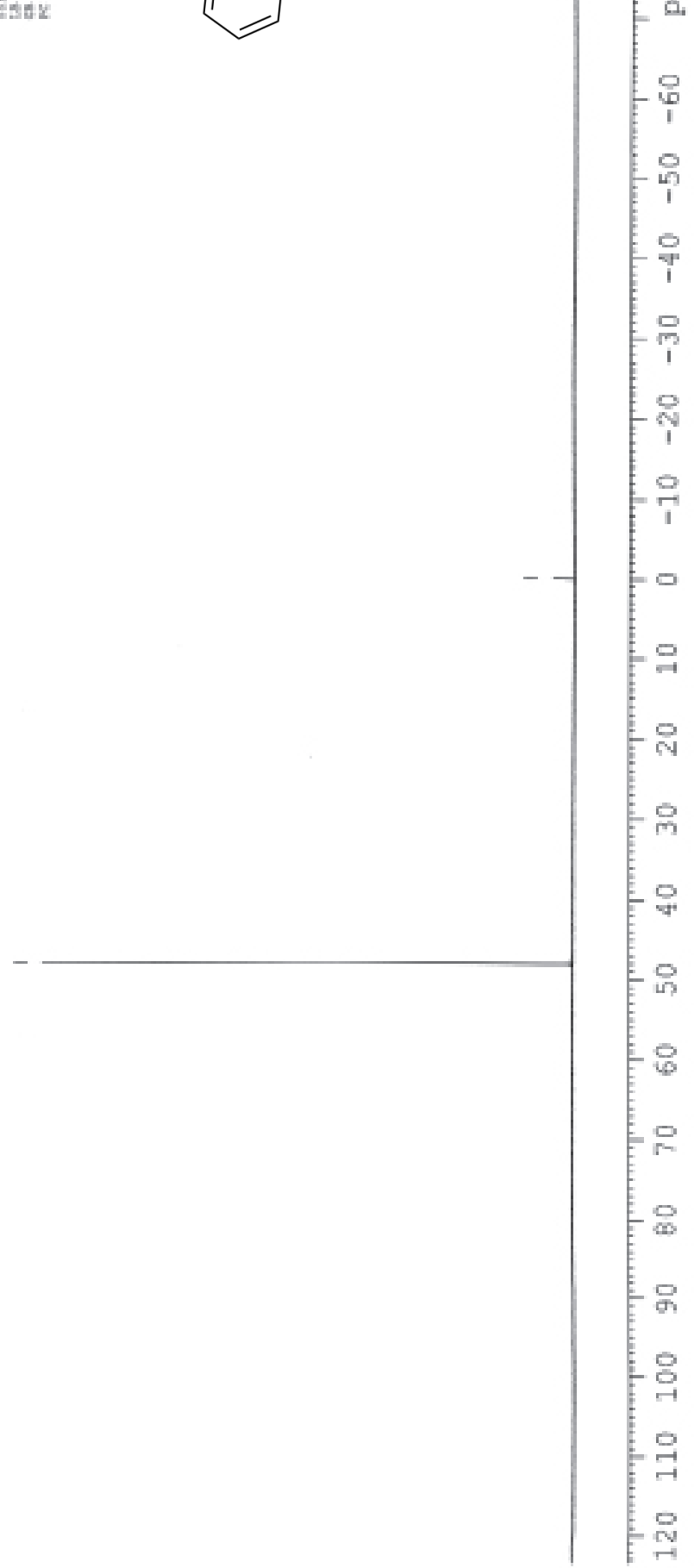


${ }^{1} \mathrm{H}$ NMR Spectrum of 2-Fluoro-3-(2-bromophenylsulfanyl)benzo[b]thieno[3,2- $\left.d\right]$ thiophene 30

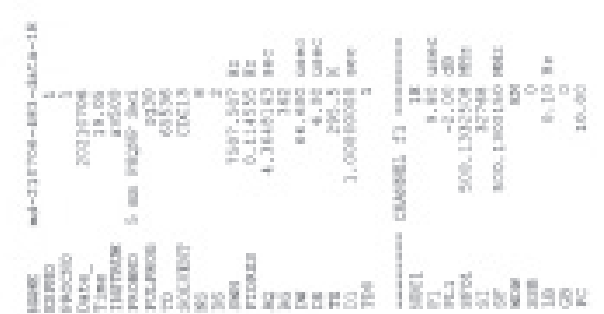<smiles>Cc1oc2oc3ccccc3c2c1Oc1ccccc1Cl</smiles>

웅
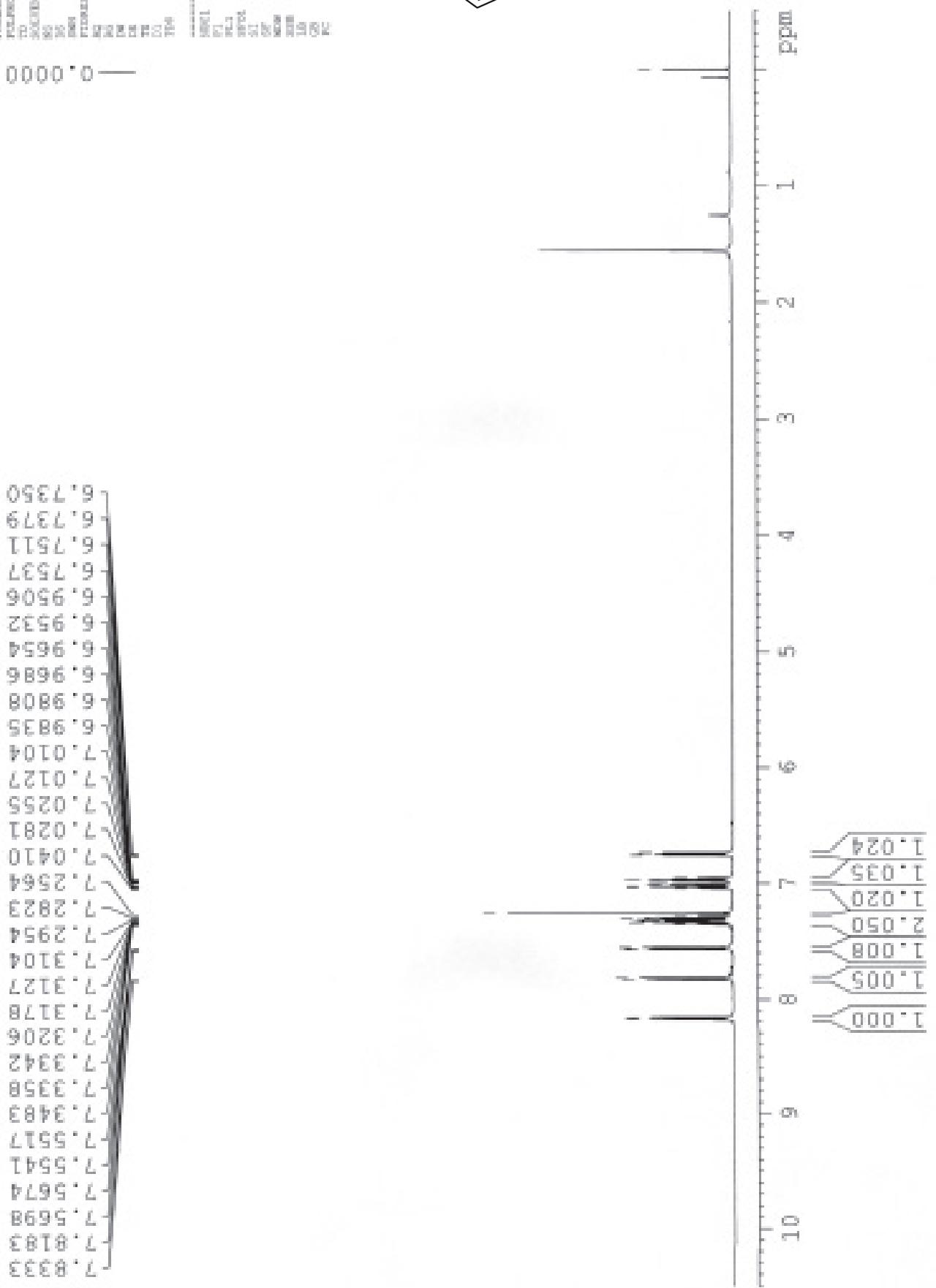
${ }^{13} \mathrm{C}$ NMR Spectrum of 2-Fluoro-3-(2-bromophenylsulfanyl)benzo[ $\left.b\right]$ thieno[3,2- $\left.d\right]$ thiophene 30

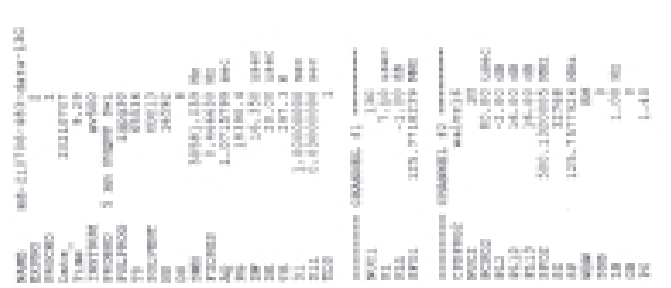<smiles></smiles>

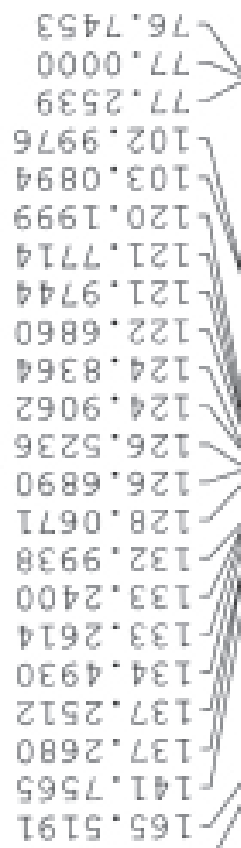<smiles>C1C2CC1[C@H]1CC[C@H]21</smiles>
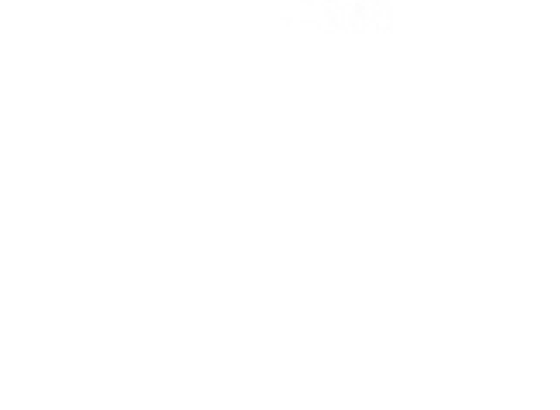
${ }^{19}$ F NMR Spectrum of 2-Fluoro-3-(2-bromophenylsulfanyl)benzo[b]thieno[3,2- $\left.d\right]$ thiophene 30

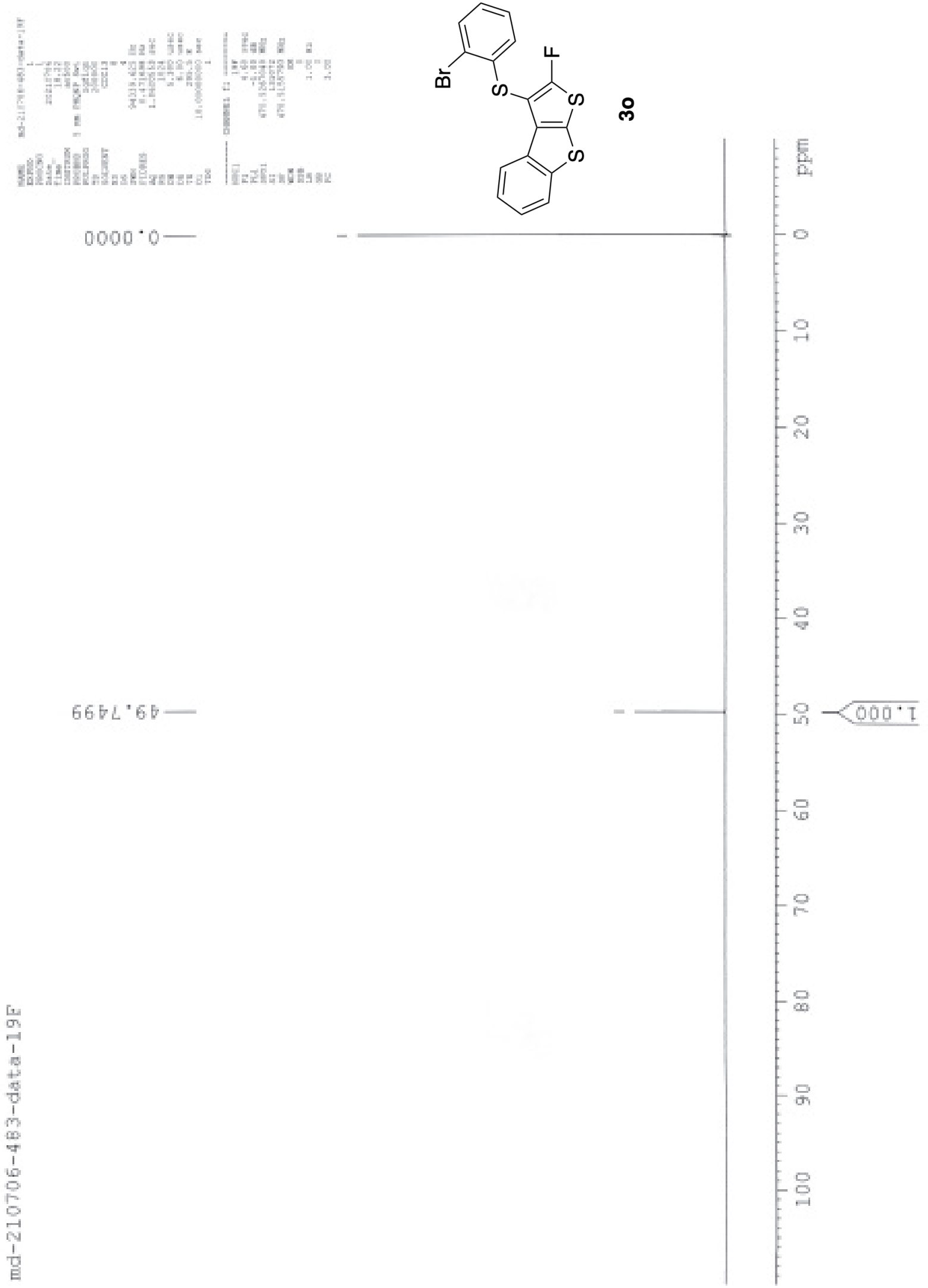


${ }^{1} \mathrm{H}$ NMR Spectrum of 1,1-Difluoro-2-(phenylsulfanyl)-2-(2-thienyl)ethene 4a

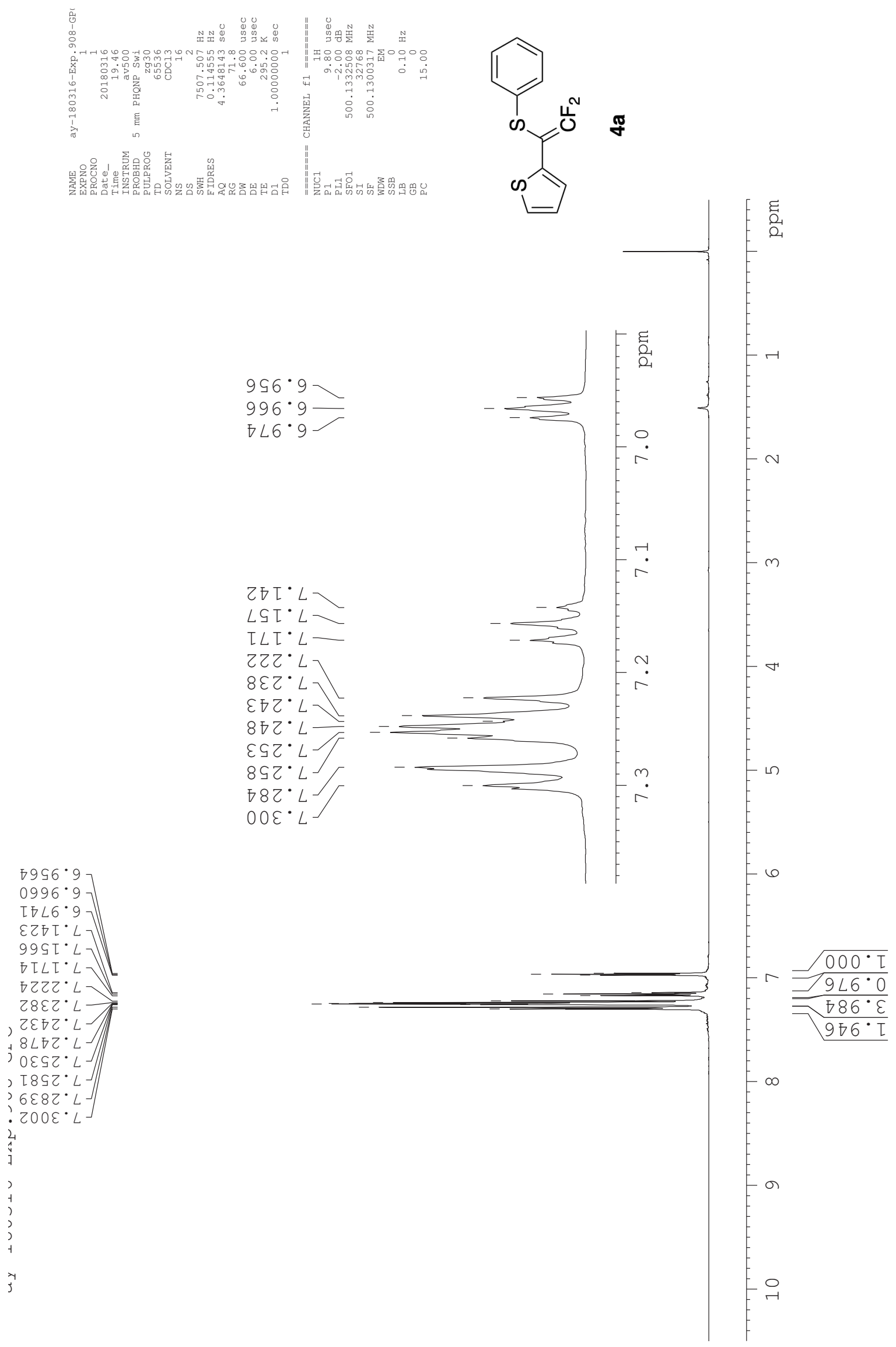


${ }^{13} \mathrm{C}$ NMR Spectrum of 1,1-Difluoro-2-(phenylsulfanyl)-2-(2-thienyl)ethene 4a
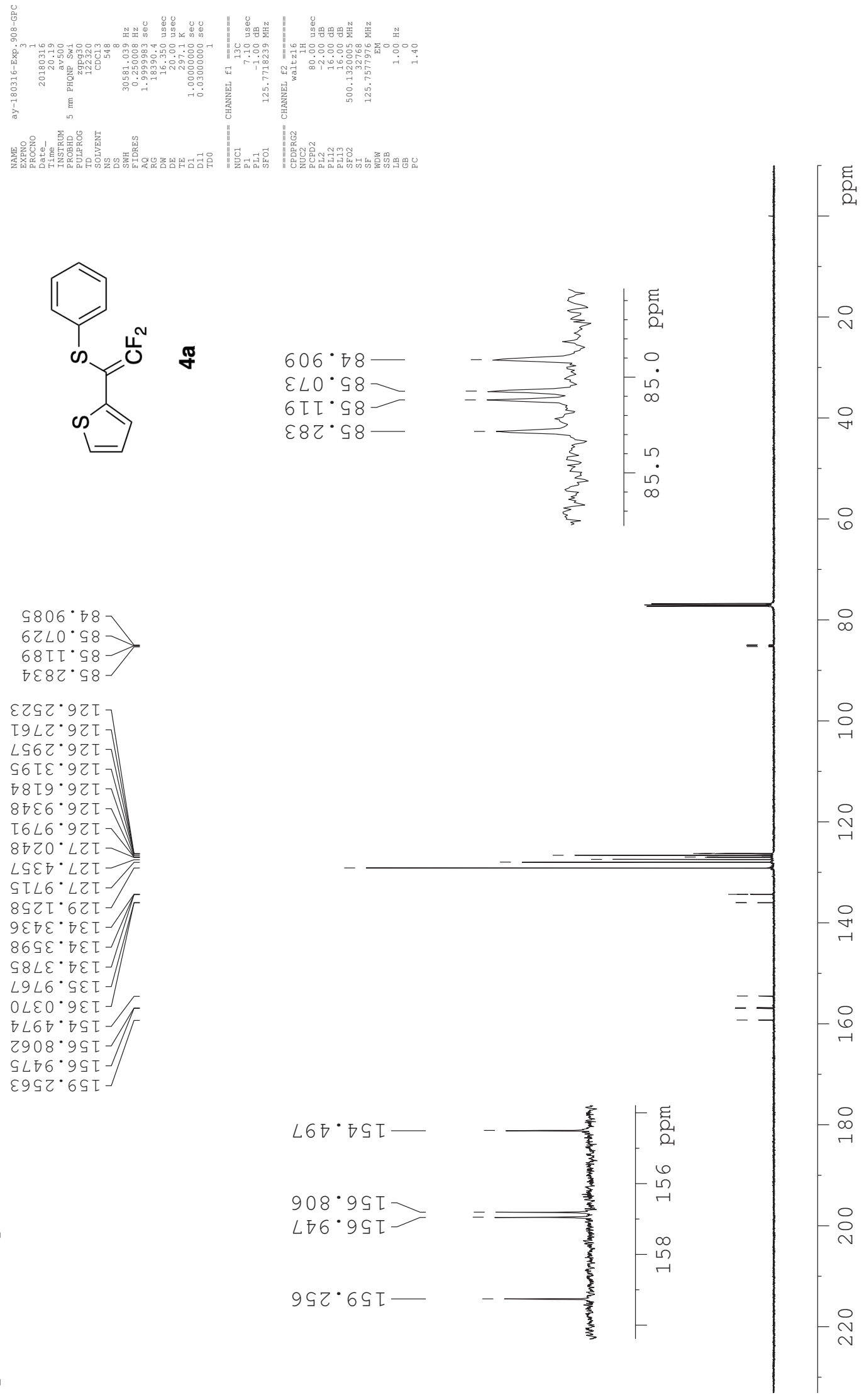

$9806^{\circ}$ เ8

$62 \angle 0 \cdot 98$

$68 \mathrm{II} \cdot \mathrm{s} 8$

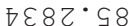

हZSて・9ZT

[9LZ・9ZI]

$\angle S 6 Z \cdot 9 Z[$

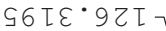

78โ9.9Zโ

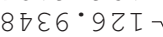

[6L6.9Z[]

$8 \nabla Z 0^{\circ} L Z T$

$\angle S E{ }^{\circ} \angle Z T$

SIL6 $\angle Z T$

8SZI.6ZI

$9 \varepsilon \nabla \varepsilon \cdot \nabla \varepsilon \tau-$

$86 \varsigma \varepsilon^{\circ} \nabla \varepsilon \tau-$

ऽ $L \varepsilon \cdot \nabla \varepsilon \tau-$

$\angle 9 L 6^{\circ} \mathrm{SEL}$

$0\llcorner\varepsilon 0 \cdot 9 \varepsilon T$

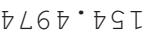

$2908^{\circ} 99$ I

SLD6.9ST

घ9G $\cdot 69 I$.

0
0
0
1
0
0
0
0
0
$x$
1
1
1
6
01
0
0
$\infty$
7
1
2
0
0 
${ }^{19}$ F NMR Spectrum of 1,1-Difluoro-2-(phenylsulfanyl)-2-(2-thienyl)ethene 4a

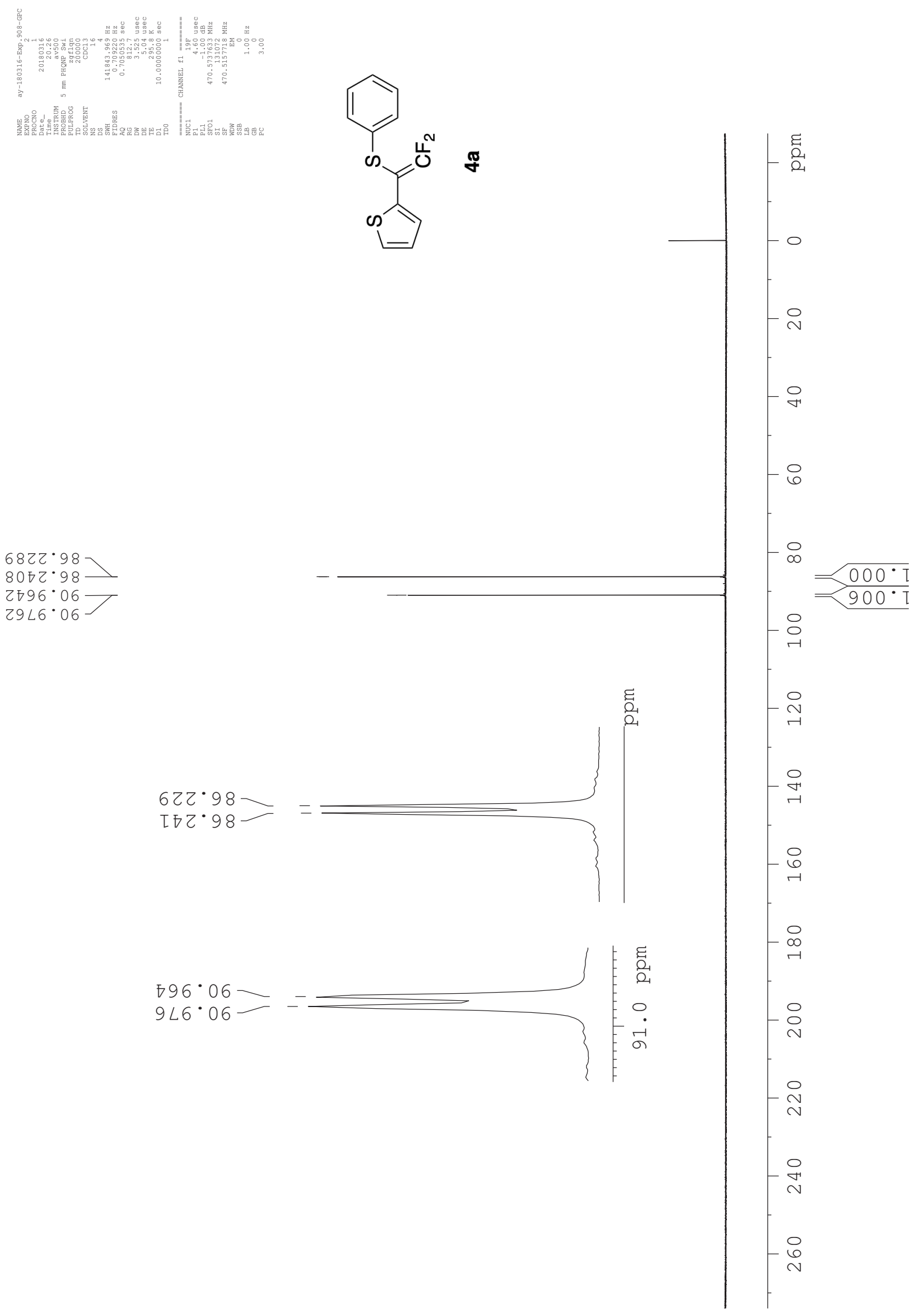


${ }^{1}$ H NMR Spectrum of Phenyl benzofuran-2-carbodithioate $6 \mathbf{a}$
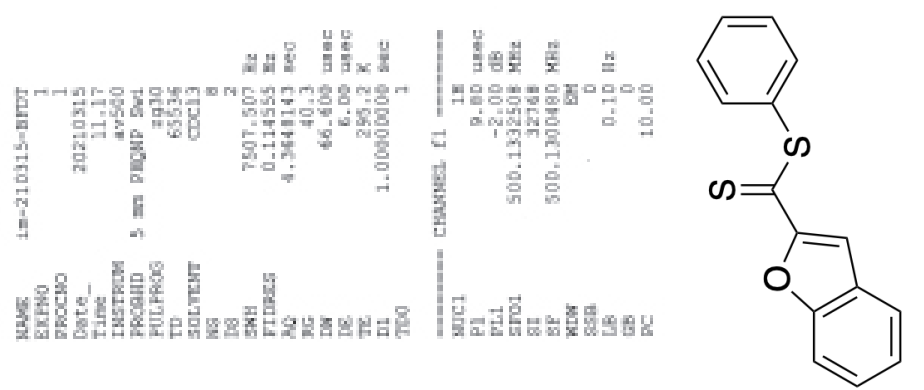

§

$0000^{\circ} 0-$

$\varepsilon 9 \tau G^{*} \tau-$
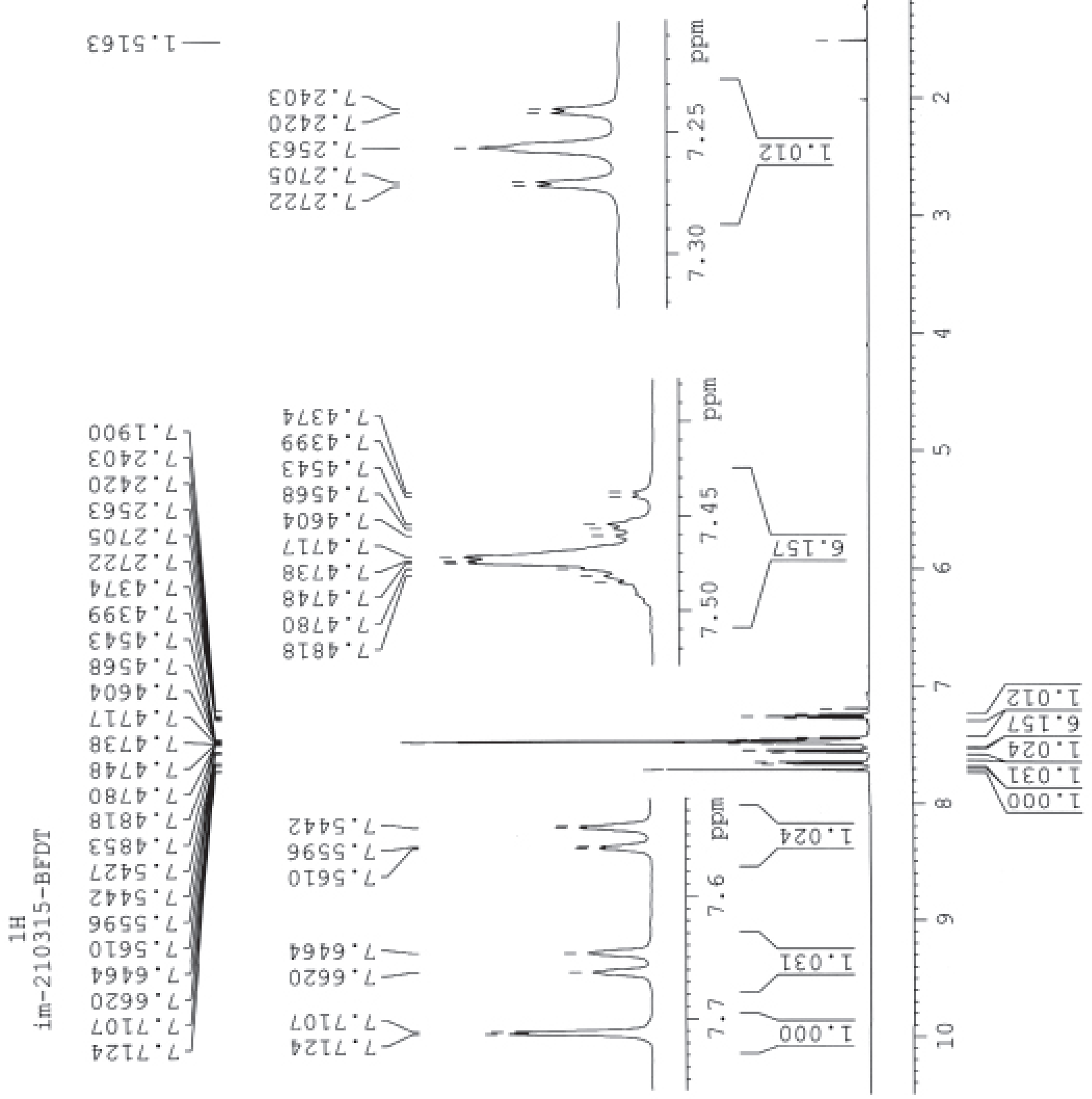
${ }^{13} \mathrm{C}$ NMR Spectrum of Phenyl benzofuran-2-carbodithioate $\mathbf{6 a}$
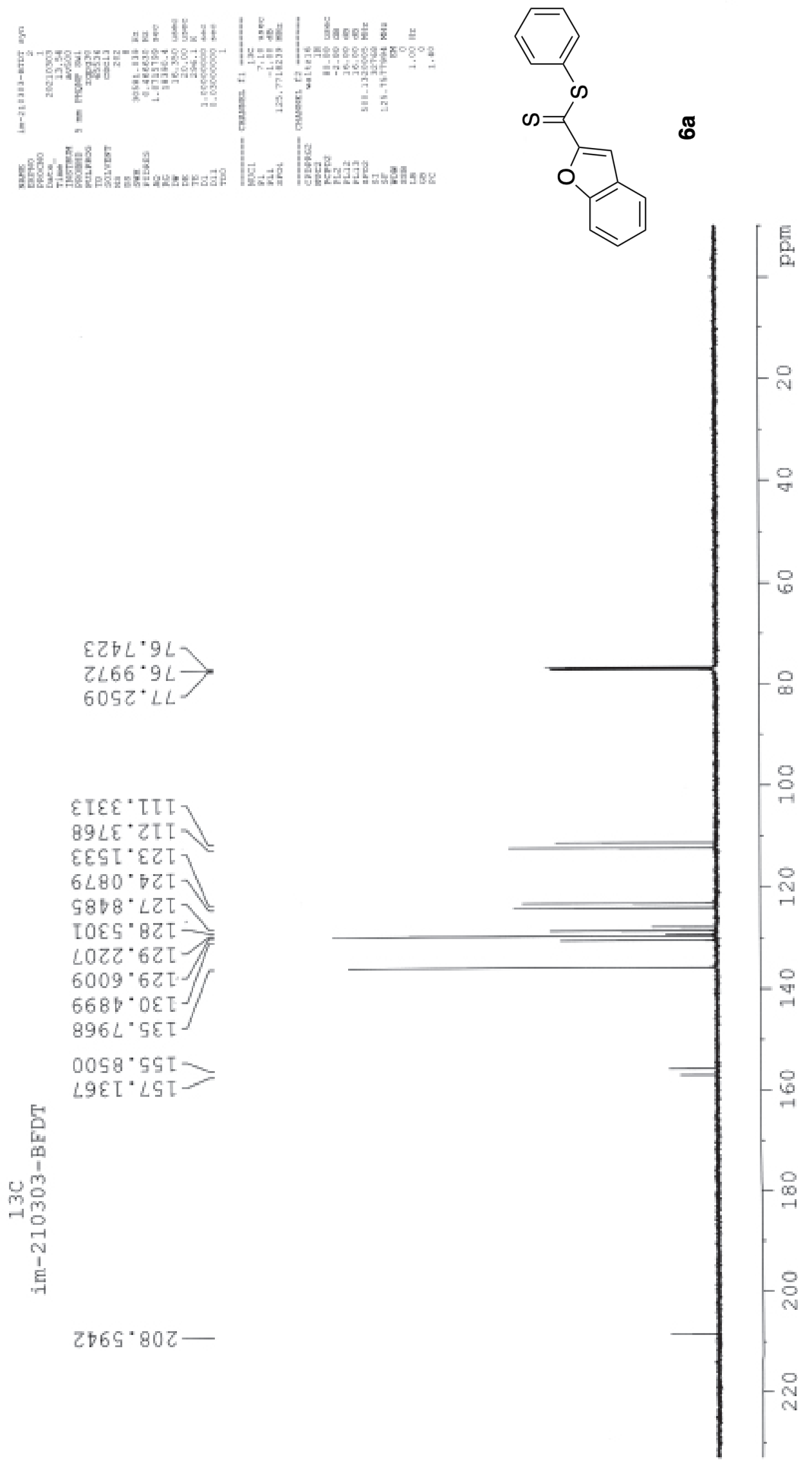
${ }^{1} \mathrm{H}$ NMR Spectrum of 2-Bromophenyl benzofuran-2-carbodithioate $\mathbf{6 b}$

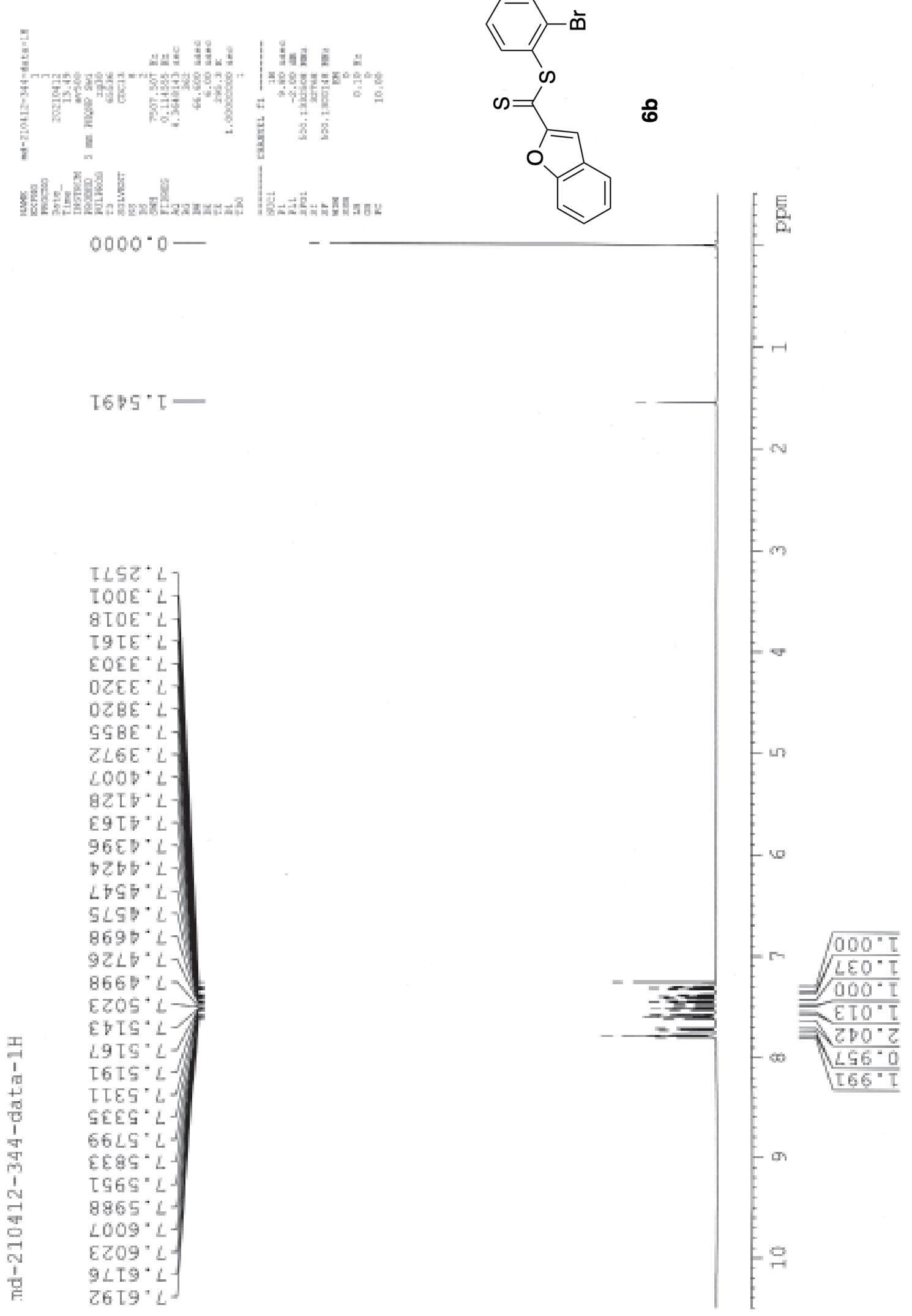


${ }^{13} \mathrm{C}$ NMR Spectrum of 2-Bromophenyl benzofuran-2-carbodithioate $\mathbf{6 b}$

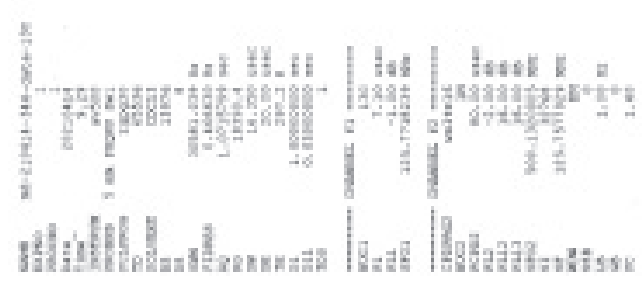<smiles>O=C(Oc1cc2c(o1)C=CCC2)c1ccccc1</smiles>

응

$8510 * 0-$

$8 \angle \forall L L^{\circ} 9 L$

$9 T 00^{\circ} \mathrm{LL}$

$2952 \cdot L L$

$5989^{\circ}$ เIT.

عOSt.ZIL]

BEOZ*EZL,

$\varepsilon 0 \angle T^{\circ} \forall Z T$,

ZLO6. $\angle Z L-$

9E86*8ZL

ZT $\angle 9^{\circ} 8 Z \tau$

ร०T $0^{\circ} 0 \varepsilon \mathrm{L}$

$9 Z 00^{\circ} \tau \varepsilon \tau$

ZSLT'ZEIJ

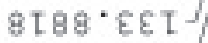

y $\angle 260^{\circ} 8 \varepsilon T$ I

ร9\$6. $55 \mathrm{I}$

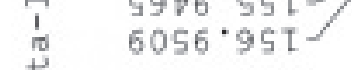

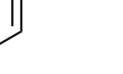

是

家

宣

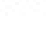

tis

$\frac{1}{9}$

$\stackrel{1}{\stackrel{1}{2}}$

学

站 $09 \pi \cdot$ t 
${ }^{1}$ H NMR Spectrum of 2-Fluoro-3-(phenylsulfanyl)thieno[3,2-b]benzofuran 7a

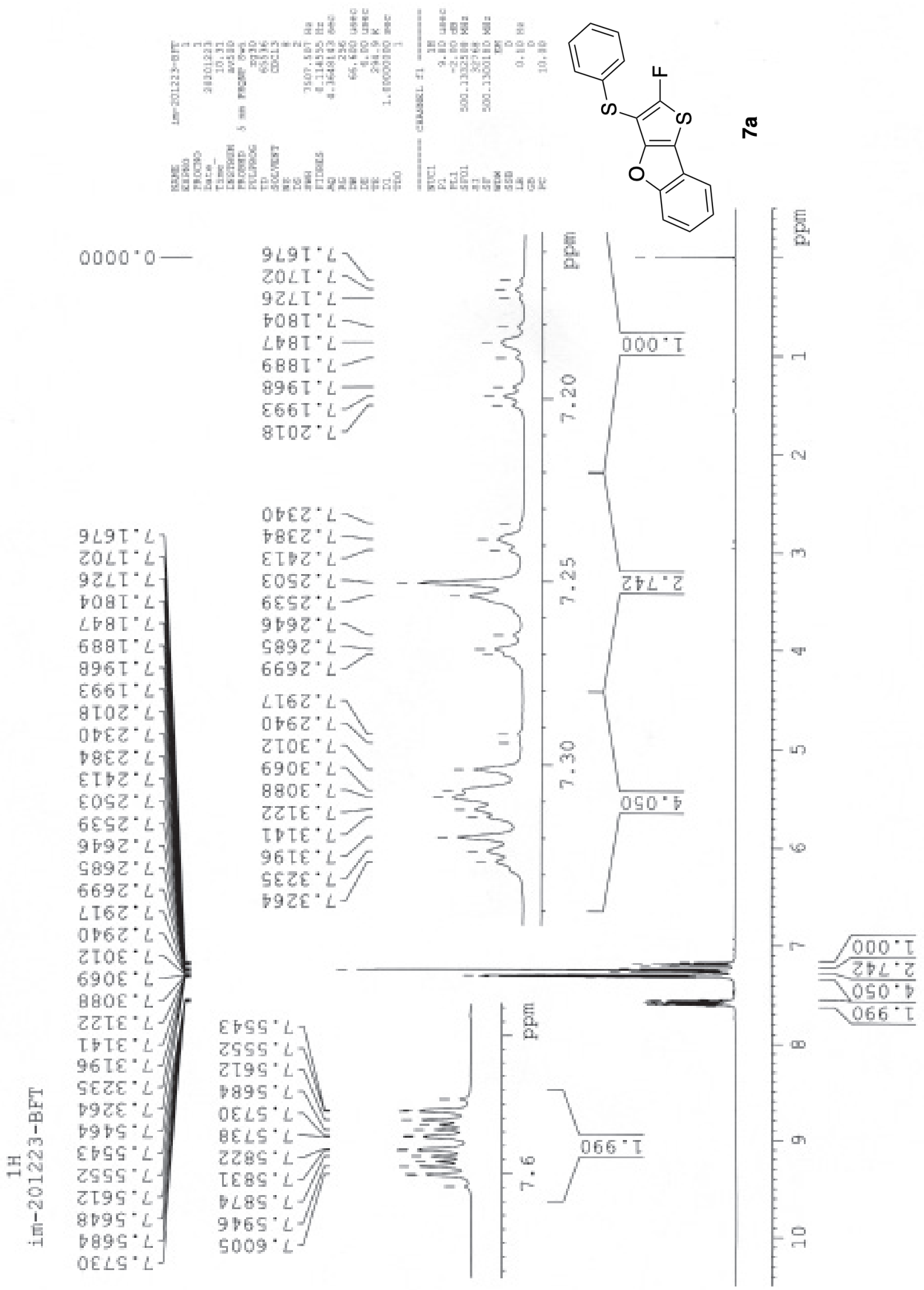


${ }^{13} \mathrm{C}$ NMR Spectrum of 2-Fluoro-3-(phenylsulfanyl)thieno[3,2-b]benzofuran $7 \mathbf{a}$
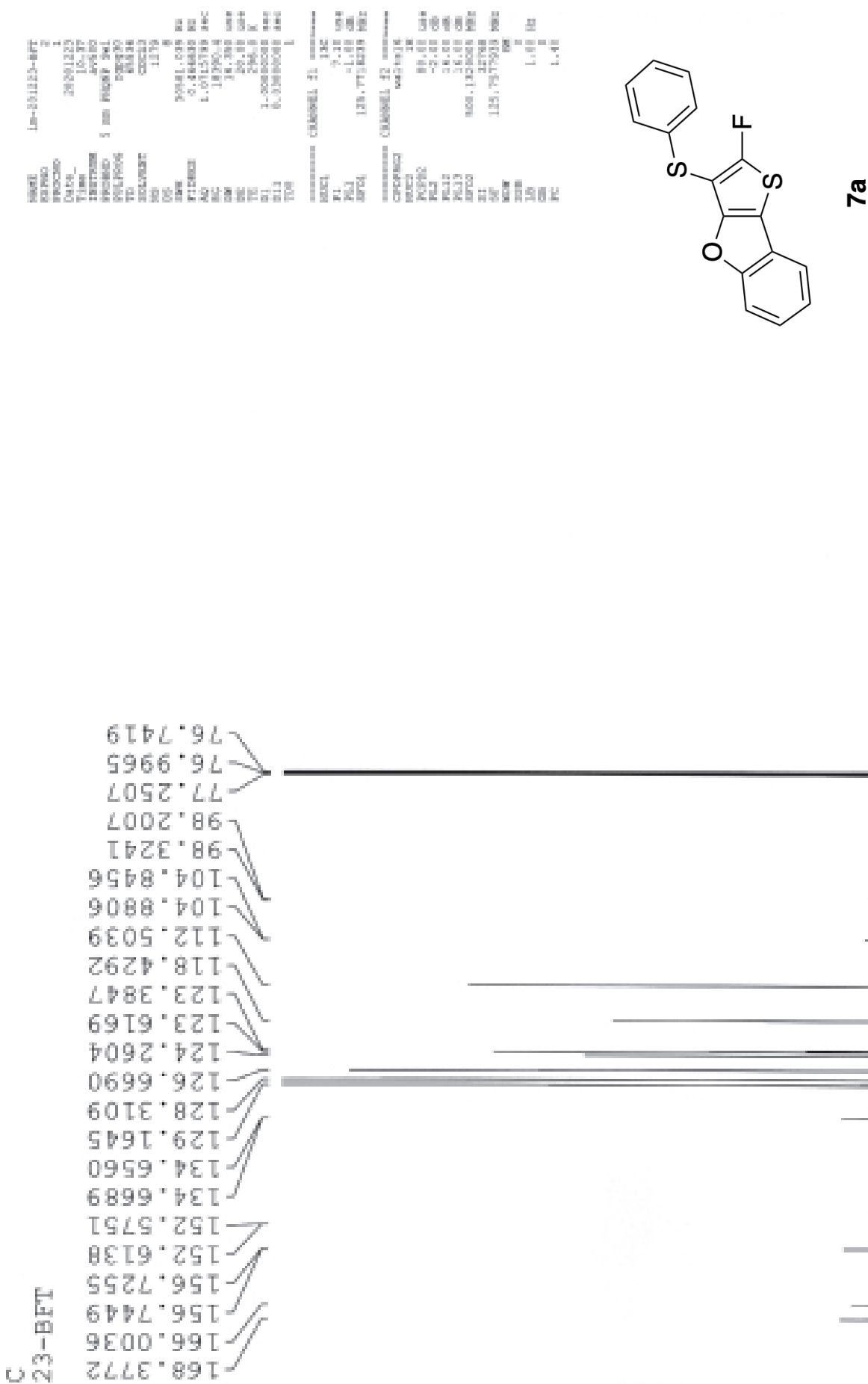
${ }^{19}$ F NMR Spectrum of 2-Fluoro-3-(phenylsulfanyl)thieno[3,2-b]benzofuran $7 \mathbf{a}$
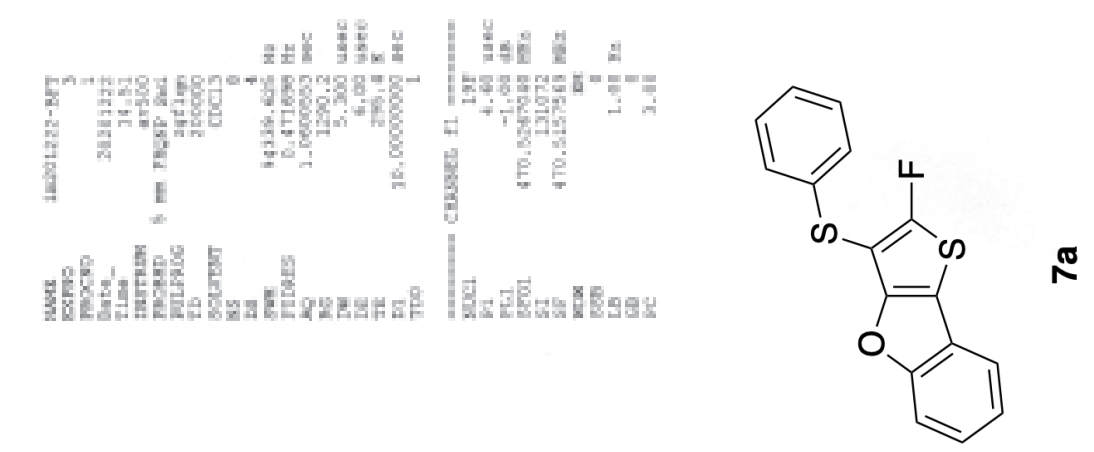

$1000^{\circ} 0-$

$0265 \cdot 85-$

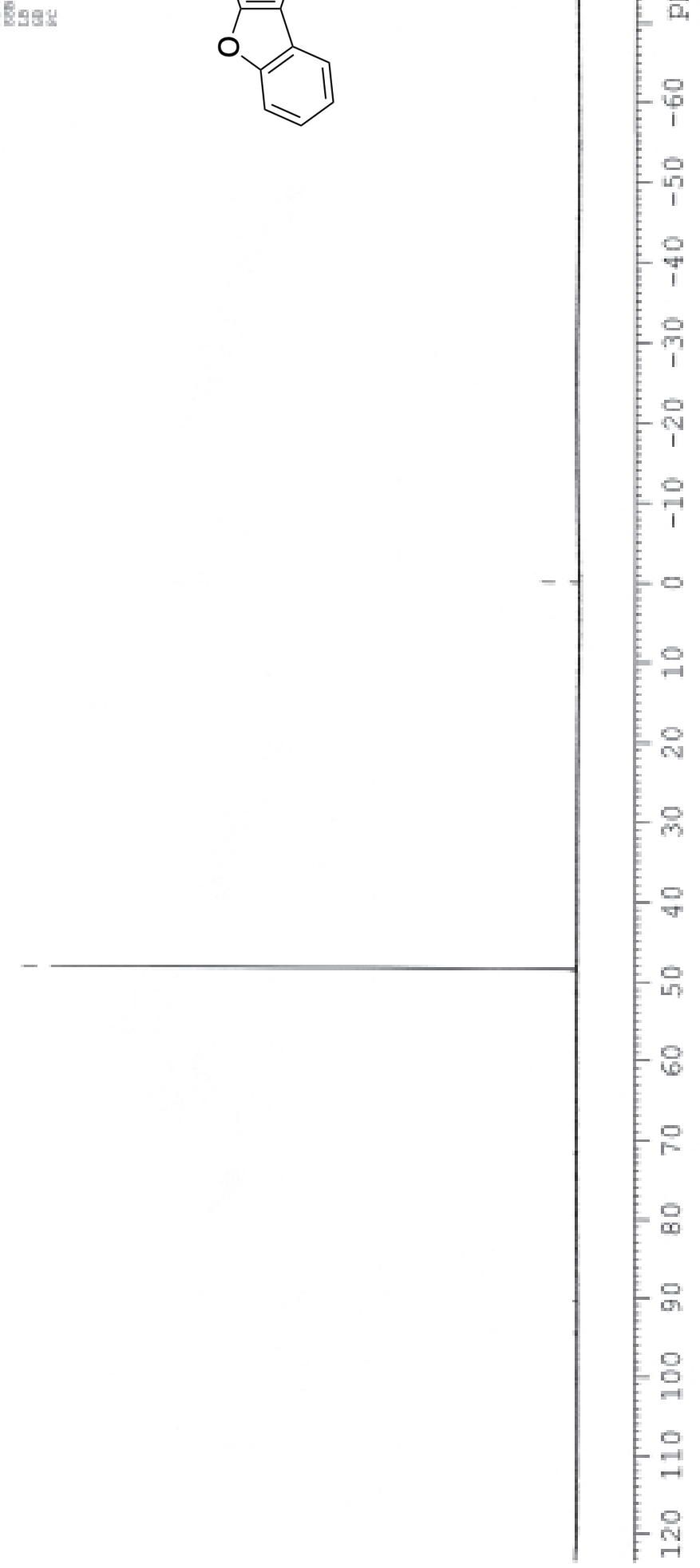


${ }^{1}$ H NMR Spectrum of 2-Fluoro-3-[(2-bromophenyl)sulfanyl)]thieno[3,2-b]benzofuran $7 \mathbf{b}$

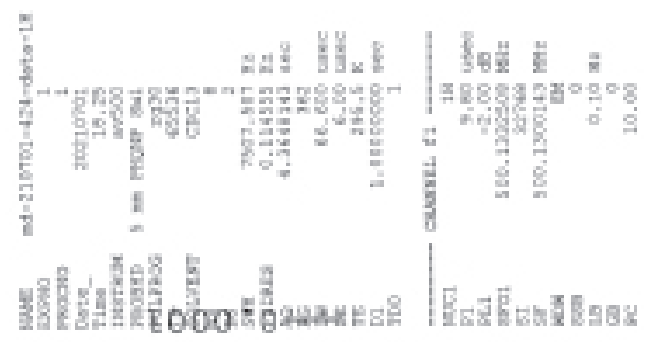<smiles>Cc1oc2c(oc3ccccc32)c1Oc1ccccc1Br</smiles>

순
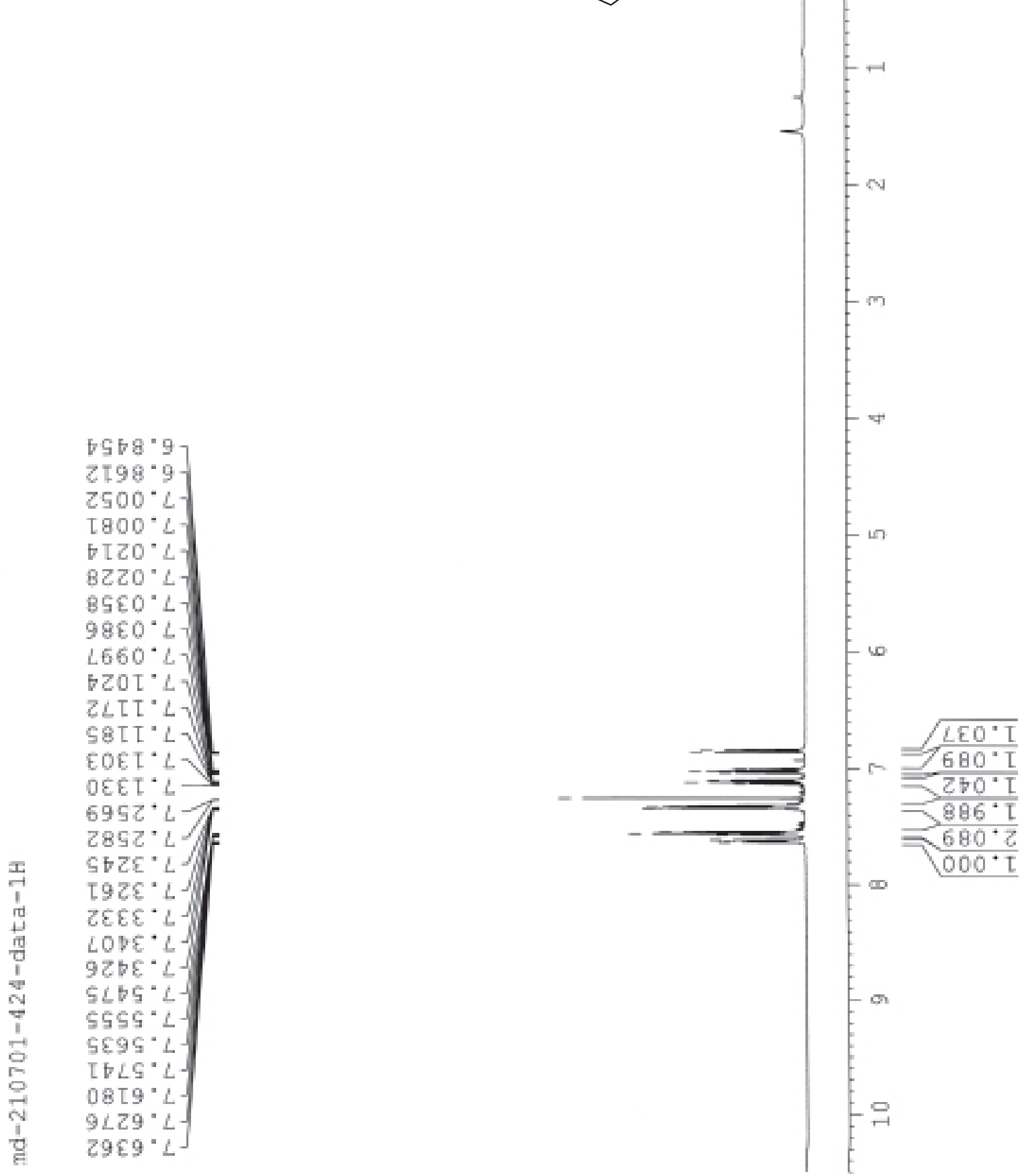
${ }^{13}$ C NMR Spectrum of 2-Fluoro-3-[(2-bromophenyl)sulfanyl)]thieno[3,2-b]benzofuran $7 \mathbf{b}$
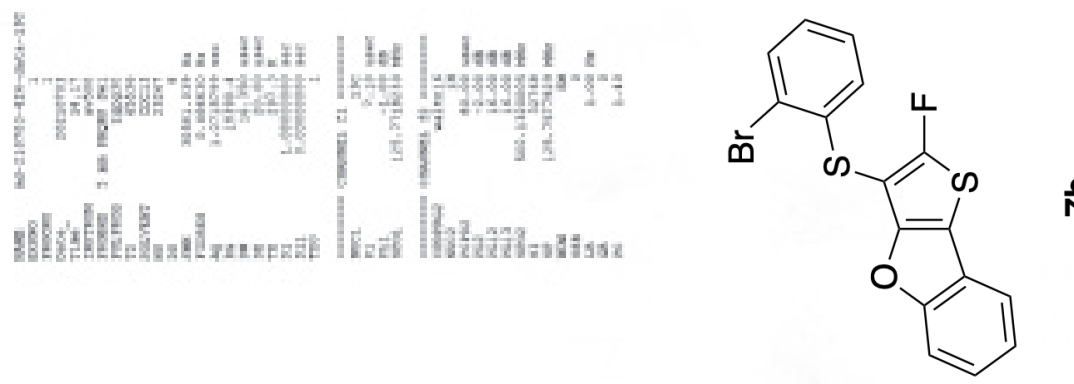

우

ES8S"ZIT.

E960.8II

$8266^{\circ} 0 Z \mathrm{Z}$

9 เ० ${ }^{\circ} \varepsilon Z \tau$

$B 6 Z L \cdot E Z L$

$6096^{\circ} \mathrm{7ZT}$

$6 \angle 60^{\circ}$ LZT

$B Z 96^{\circ} \angle Z I$

$9 \varepsilon 80^{\circ} \varepsilon \varepsilon \tau$

LOTE. $9 \varepsilon โ-$

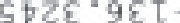

I592. Z5I

U $280 \varepsilon^{\circ} \mathrm{ZST}$

$\rightarrow$ ES8L.95T-

ESOB-9GI

ZZSL.99โ

L0t [.69T.

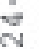

8

m.

定

$\stackrel{0}{\rightarrow}$

$\overrightarrow{\mathrm{C}}$

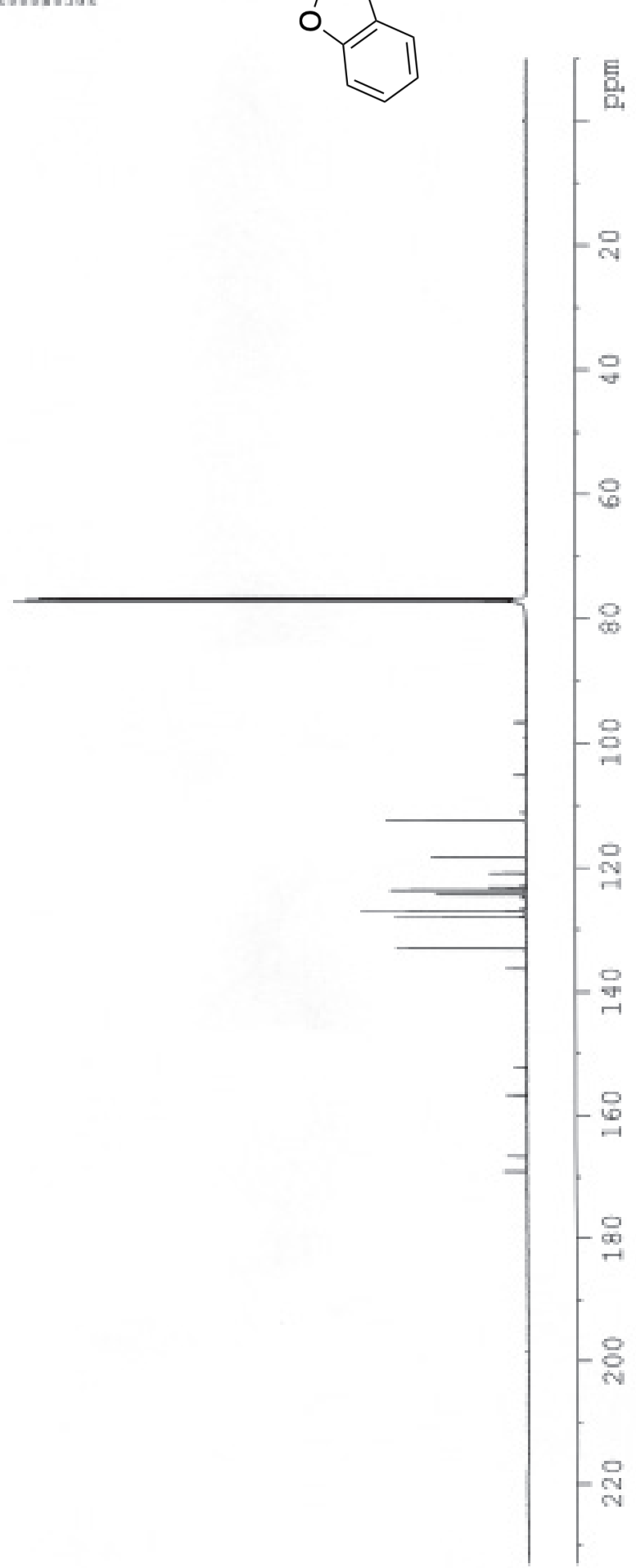


${ }^{19}$ F NMR Spectrum of 2-Fluoro-3-[(2-bromophenyl)sulfanyl)]thieno[3,2-b]benzofuran $7 \mathbf{b}$

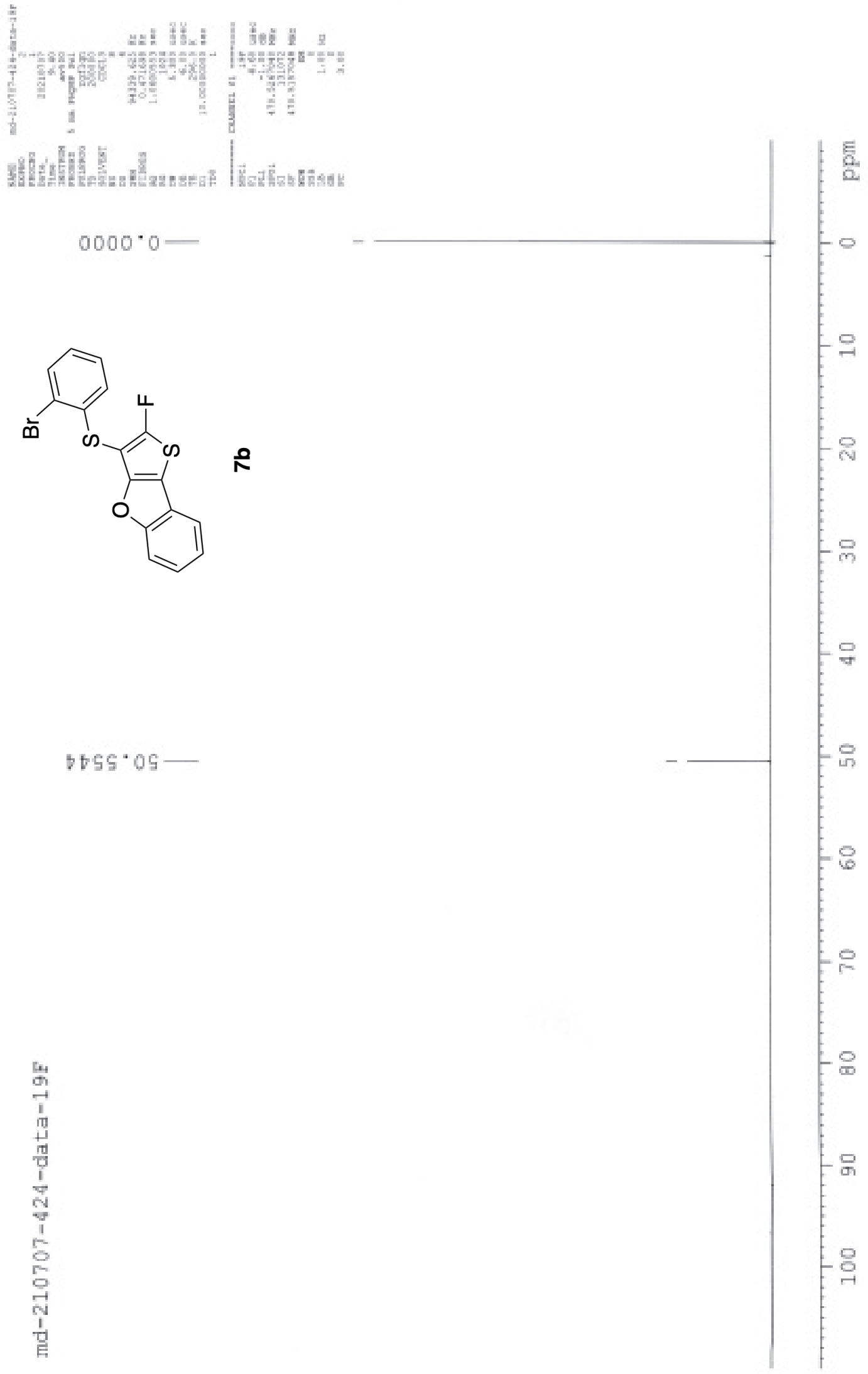



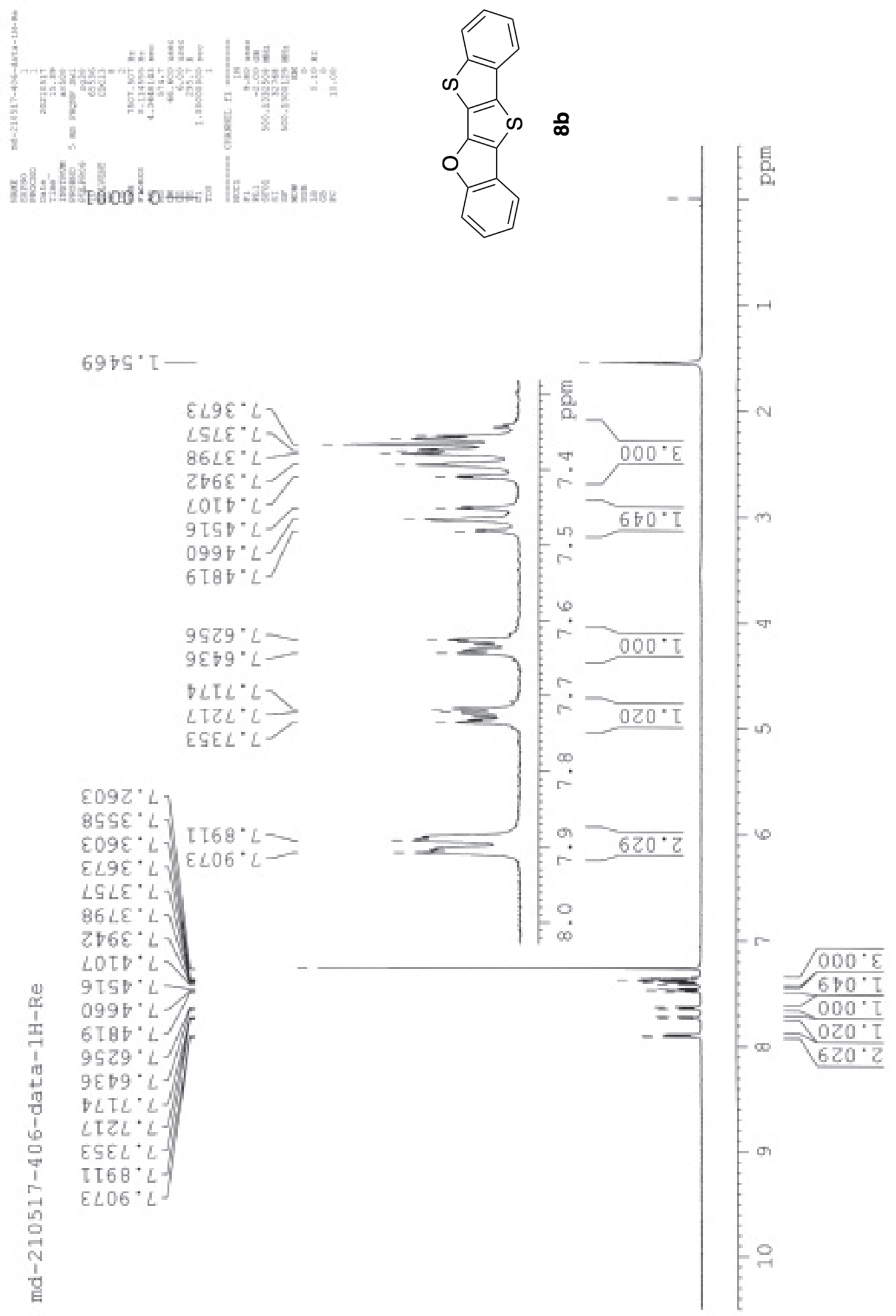
${ }^{13} \mathrm{C}$ NMR Spectrum of Benzo[4',5']thieno[2',3':4,5]thieno[3,2-b]benzofuran $\mathbf{8 b}$
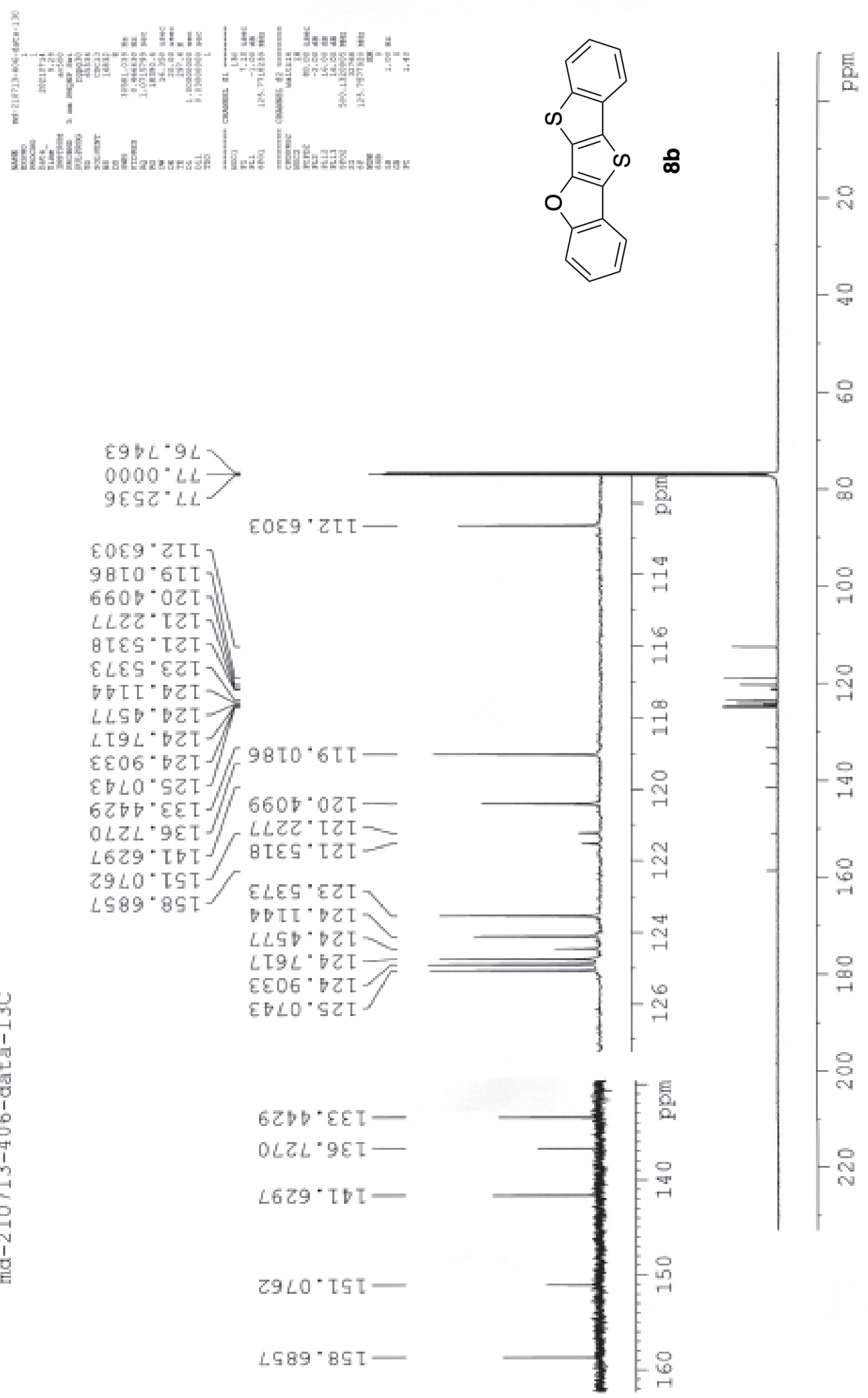